\title{
FACULTAD DE CIENCIAS DE LA ACTIVIDAD FÍSICA Y DEL DEPORTE
}

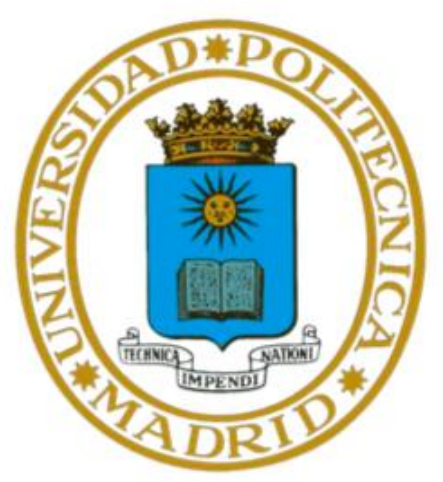

Análisis de los efectos de un programa de readaptación y reentrenamiento sobre la lesión muscular a nivel proximal del bíceps femoral en futbolistas de élite.

\section{TESIS DOCTORAL}

D.SERGIO JIMÉNEZ RUBIO

Licenciado en Ciencias de la Actividad Física y del Deporte 


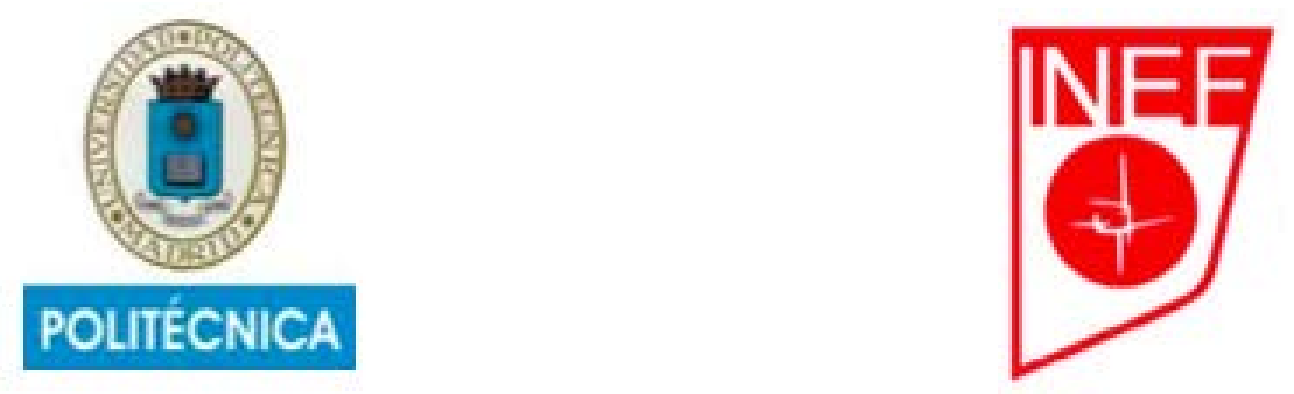

\author{
UNIVERSIDAD POLITÉCNICA DE MADRID \\ FACULTAD DE CIENCIAS DE LA ACTIVIDAD FÍSICA Y \\ DEL DEPORTE
}

Análisis de los efectos de un programa de readaptación y reentrenamiento sobre la lesión muscular a nivel proximal del bíceps femoral en futbolistas de élite.

TESIS DOCTORAL

D.SERGIO JIMÉNEZ RUBIO

Licenciado en Ciencias de la Actividad Física y del Deporte 

FACULTAD DE CIENCIAS DE LA ACTIVIDAD FÍSICA Y DEL DEPORTE

\title{
UNIVERSIDAD POLITÉCNICA DE MADRID
}

\section{Título}

Análisis de los efectos de un programa de readaptación y reentrenamiento sobre la lesión muscular a nivel proximal del bíceps femoral en futbolistas de élite.

\author{
Autor \\ D.SERGIO JIMÉNEZ RUBIO \\ Licenciado en Ciencias de la Actividad Física y del Deporte
}

\section{Directores}

DR. D. JESÚS RIVILLA GARCÍA. Doctor en Ciencias de la Actividad Física y del Deporte

DR. D. VÍCTOR PAREDES HERNÁNDEZ. Doctor en Ciencias de la Actividad Física y del Deporte

Madrid, 2019 



\section{TRIBUNAL DE LA TESIS}

Tribunal nombrado por el Mgfico. y Excmo. Sr. Rector de la Universidad Politécnica de Madrid el día,

PRESIDENTE D./Dña.

VOCAL D./Dña.

VOCAL D./Dña.

VOCAL D./Dña.

SECRETARIO/A D./Dña.

Realizado el acto de defensa y lectura de la Tesis el día,

en

CALIFICACIÓN

EL PRESIDENTE

LOS VOCALES

EL SECRETARIO 

"El mundo está en las manos de aquellos que tienen el coraje de soñar y correr el riesgo de vivir sus sueños"

\section{Paulo Coelho}





\section{AGRADECIMIENTOS}

Éste ha sido un largo y duro recorrido. Sin embargo, al echar la vista atrás, soy consciente ahora de valorar más los momentos gratificantes que las situaciones complicadas que se fueron dando durante el camino.

En primer lugar, mi agradecimiento a la Facultad de Ciencias de la Actividad Física y el Deporte, que desde 1997 ha supuesto para mí, ser un lugar especial y único.

Gracias al Getafe Club de Fútbol, por darme la oportunidad, desde el 2005, de poner en práctica conocimientos y propuestas sobre la readaptación y reentrenamiento con sus jugadores lesionados del primer equipo en el más alto nivel y con ello haber podido implementar el programa de readaptación desarrollado en esta tesis doctoral. Agradecer también a los futbolistas del primer equipo, en los diferentes años, por haberme permitido entrenarlos durante sus lesiones y dejarse aconsejar por mi. Con ello, he podido recoger datos y obtener así unos resultados y conclusiones para esta tesis doctoral que espero, sean de utilidad para futuros profesionales e investigadores. Gracias también al Rayo Vallecano de Madrid, por ser otro club de donde se obtuvieron sujetos para la muestra, y a Víctor Paredes que, como Preparador Físico y Readaptador del equipo, colaboró e implementó el programa de readaptación de la intervención.

Gracias a los entrenadores y preparadores físicos de altísimo nivel con los que he trabajado durante este proceso, con ellos he tenido el gran privilegio de compartir situaciones tan constructivas como únicas. El estrés y la urgencia del deporte de competición en la élite unido a las lesiones generan situaciones complejas cuya gestión requiere conocimientos y experiencias específicas.

Gracias a amigos y compañeros del ámbito del entrenamiento y la readaptación de las lesiones por animarme a investigar y a ser constante sobre este tema: Juan Ribot, José Mascarós, Oscar García, José Romero, Josté Vallejo, Fermín Valera, José Luis Estévez, Víctor Paredes. 
Gracias a Carlos Lalín y Xavi Peirau, dos amigos y excelentes profesionales, referentes nacionales e internacionales en el ámbito del entrenamiento con el deportista lesionado. Ellos nos mostraron el camino y la nomenclatura para aprender sobre la importancia de la readaptación de lesiones y la salud de los deportistas, siempre a través del ejercicio.

Por supuesto, a mis directores de tesis doctoral, Jesús y Víctor, excelentes profesionales, que han sabido guiarme magistralmente por las diferentes etapas de este proyecto, etapas que sin vosotros hubieran sido un imposible. He aprendido, mejorado y disfrutado con vuestras orientaciones, correcciones, apoyos y consejos magistrales.

Gracias también a mi amigo Archit Navandar, su ayuda y dedicación ha sido determinante tanto en esta tesis doctoral como en los artículos publicados sobre la misma.

Gracias al profesor Miguel Ángel Gómez Ruano por tenderme la mano y ayudarme a comprender la metodología de investigación así como conceptos de análisis de importante relevancia.

Gracias al profesor Manuel Sillero Quintana, por ser mi amigo y por haber estado siempre ahí durante este camino desde la universidad, mostrando un gran compromiso para que esta tesis doctoral avanzase en cada momento.

Gracias al profesor Rubén Barakat Carballo, por hacer muy bien su trabajo, y facilitar el proceso, las gestiones y optimizar las actuaciones para que el proyecto finalmente llegase a consumarse en una lectura oficial.

A Mari Carmen, mi mujer, mi compañera de toda la vida, gracias por estar ahí siempre; gracias por animarme a continuar incluso en momentos donde yo no atisbaba ninguna posibilidad de avanzar. Esta tesis doctoral es posible por tu dedicación a la familia, porque has dedicado más tiempo a los demás que a ti misma, porque incluso en tus momentos más duros - y aún debiendo afrontar una dura enfermedad - has estado ahí, aportándonos a todos la energía y voluntad necesarias para seguir luchando. Eres admirable. 
Quiero agradecer a mis dos hijos, Pablo y Gonzalo, ahora con 7 y 4 años. Porque este largo camino me ha hecho ser plenamente consciente del gran valor de disfrutar de vosotros.

A Tomás y Carmeli, mis padres, y a Tomás, mi hermano, por darme constantes oportunidades y a la vez enseñarme lo que es el respeto y el concepto de responsabilidad para aprovechar el tiempo y las ocasiones durante la vida.

A mi suegra Mari, por la cantidad de horas que ha estado cuidando y guiando a mis hijos, sus nietos. Gracias, porque mucho tiempo he podido dedicar a trabajar en esta tesis doctoral, con la tranquilidad de saber que estaban en buenas manos.

Finalmente, quiero agradecer a todas las personas e instituciones que han colaborado de alguna forma para ayudarme a completar esta enriquecedora etapa de mi vida, que ha desembocado en una tesis doctoral, espero que fructífera para el futuro.

Muchísimas gracias a todos. 
ÍNDICE

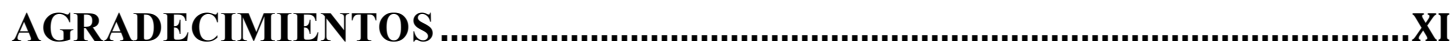

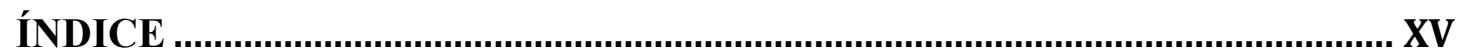

Índice de Tablas ...............................................................................................

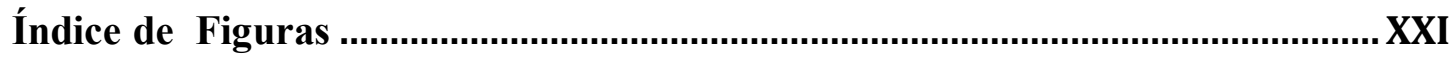

Índice de Abreviaturas ................................................................................. XXIV

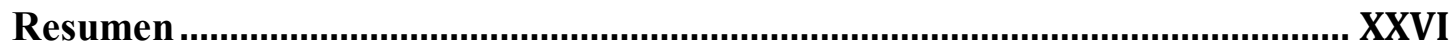

Abstract

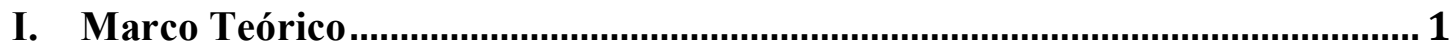

1.1 Introducción ..............................................................................................................

1.2 Antecedentes y estado de la cuestión..................................................................4

1.3 Justificación ............................................................................................................8

1.4 Delimitación del problema. Epidemiología de lesión .....................................11

1.4.1 Lesiones de isquiosurales en deportistas amateurs/profesionales. ...........13

1.4.2 Lesiones de isquiosurales en el fútbol profesional .....................................14

1.4.3 Recidivas y consecuencias de las lesiones de isquiosurales. .....................15

1.5 Grupo muscular isquiosural: características y función .................................17

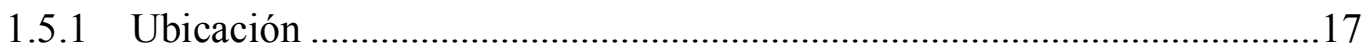

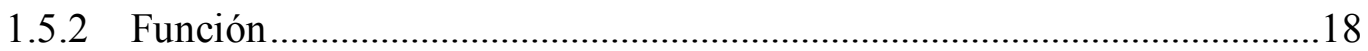

1.5.3 Cinemática de la carrera en relación a los isquiosurales...........................19

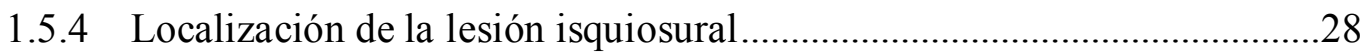

1.5.5 Causas de lesión. Mecanismos lesionales .....................................................33

1.5.5.1 El sprint como mecanismo de lesión ........................................................34

1.5.5.2 El golpeo como mecanismo de lesión ...................................................35

1.5.5.3 Otros mecanismos de lesión ..................................................................36

1.6 Estrategias preventivas ....................................................................................38

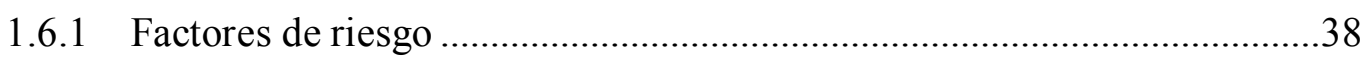

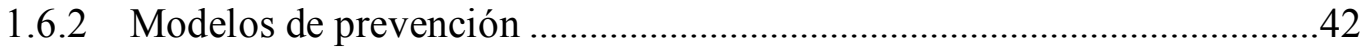

1.6.3 Programas de rehabilitación / readaptación en lesiones isquiosurales .....48 
1.7 La herramienta de medición del estudio: el GPS.........................................52

1.7.1 ¿Por qué monitorizar la carga de entrenamiento? ...................................53

1.7.2 Medios de control de la carga de entrenamiento .....................................55

1.7.3 Utilización de tecnología GPS para el control del riesgo de lesión ..........56

1.7.4 Utilización de tecnología GPS para el control en readaptación ...............56

1.7.5 Aplicación del GPS en el Fútbol de alto rendimiento ..............................61

1.7.5.1 Fiabilidad/ Validez de los GPS .......................................................66

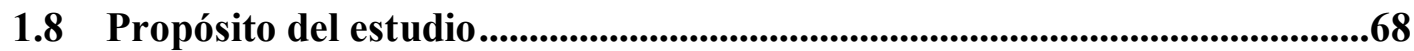

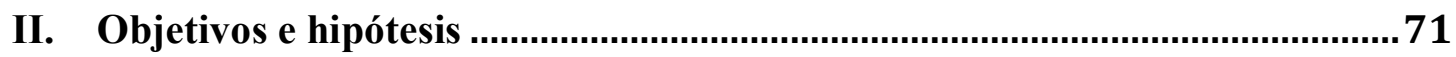

2.1 Objetivos Generales .............................................................................................................73

2.2 Objetivos Específicos .....................................................................................................74

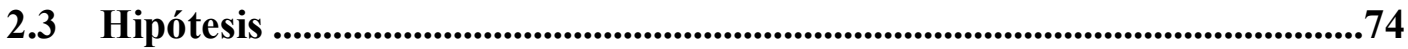

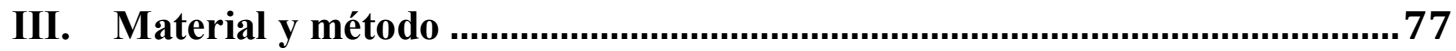

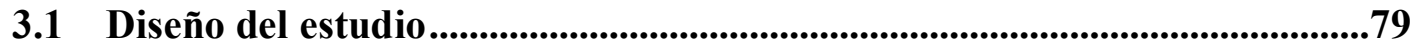

3.2 Variables del estudio .............................................................................................88

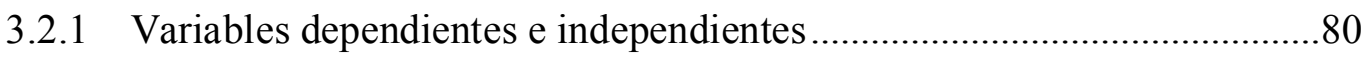

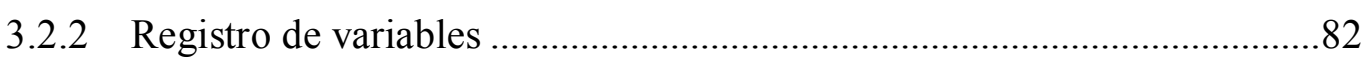

3.2.2.1 Variables $\mathrm{m} / \mathrm{min}$ a diferentes velocidades $(\mathrm{V} 1, \mathrm{~V} 2, \mathrm{~V} 3) \ldots \ldots \ldots \ldots \ldots \ldots \ldots . . . .84$

3.2.2.2 Variables Vel maxima (Max_speed), Vel media (Avg_speed) ..........85

3.2.2.3 Variable Work-to-rest ratio $(\mathrm{RD}>7.0 \mathrm{~km} / \mathrm{h} / \mathrm{RD} \leq 7.0 \mathrm{~km} / \mathrm{h})$............86

3.2.2.4 Variable $\mathrm{n}^{\mathrm{o}}$ Acel y Desacel $/ \mathrm{min}$ media intensidad $(2.9-4 \mathrm{~m} / \mathrm{s} 2) \ldots .87$

3.2.2.5 Variable $\mathrm{n}^{\mathrm{o}}$ Acel y Desacel $/ \mathrm{min}$ de alta intensidad $(>4 \mathrm{~m} / \mathrm{s} 2) \ldots \ldots \ldots \ldots . .88$

3.2.2.6 Variable $\mathrm{n}^{\mathrm{o}} \mathrm{RSA} / \mathrm{min}$ con recuperación $(<6 \mathrm{seg}$ ) y Acel $>3 \mathrm{~m} / \mathrm{s} 2$.......89

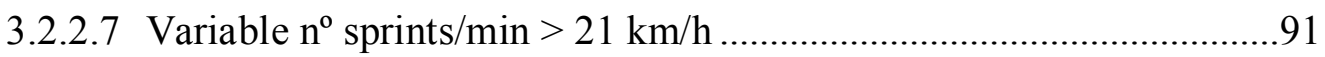

3.2.2.8 Variable Aceleración máxima ..............................................................92

3.2.2.9 Variable número de impactos $>$ 7.1g ..............................................92

3.2.2.10 Variable Explosive distance $(\mathrm{m} / \mathrm{min})$...............................................93

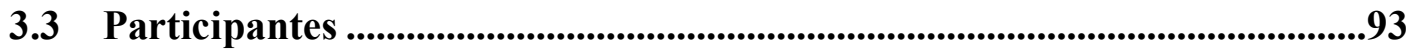

3.3.1 Perfil de lesión de los sujetos de la muestra ............................................95

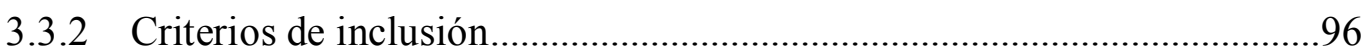

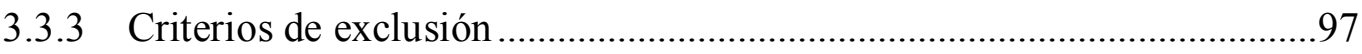

3.4 Procedimiento ................................................................................................................97 
3.5 Programa de entrenamiento durante la lesión

3.5.1 Tareas previas a iniciar programa definitivo

3.5.2 Orden y descripción de las tareas durante la intervención del estudio.. 103

3.6 Criterios utilizados para regresar al grupo y a la competición ................ 108

3.7 Validación de contenido de la intervención ...................................................... 109

3.7.1 Índice de Validez -V de Aiken con expertos ........................................ 110

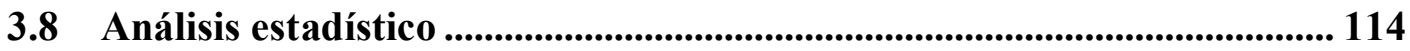

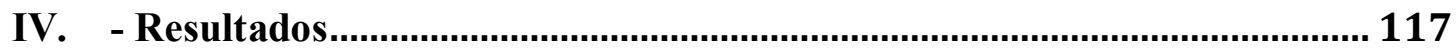

4.1 Resultados a nivel de días que tardaron en recuperarse ............................ 119

4.2 Resultados obtenidos tras comparaciones PRE-REA - GRUPO ........... 120

4.3 Resultados obtenidos tras comparaciones PRE-C1-C2 …........................ 132

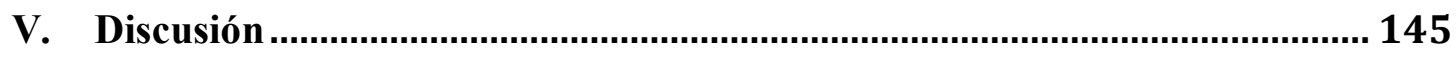

5.1 Discusión sobre resultado de días que tardaron en recuperarse............. 147

5.2 Discusión sobre resultados PRE-REA-GRUPO ...................................... 150

5.3 Discusión sobre resultados PRE-C1-C2 .................................................. 157

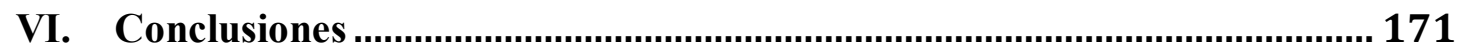

6.1 Perspectivas de investigación y futuras líneas de investigación .............. 174

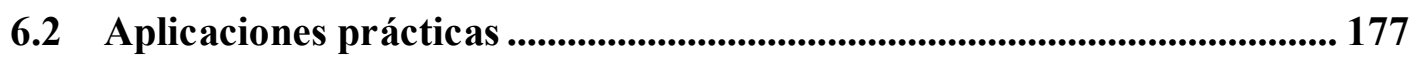

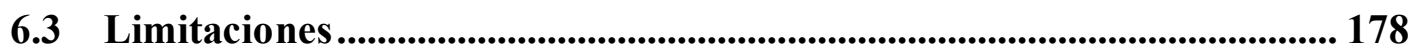

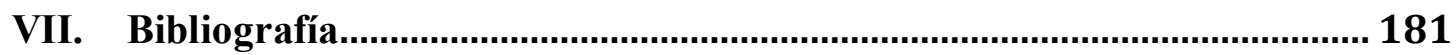

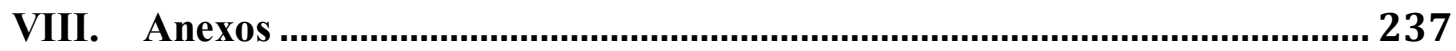

8.1 Permisos para la toma de datos ...................................................................... 239

8.2 Comité de Ética ................................................................................................... 241

8.3 Consentimiento informado para los participantes de la investigación .. 243

8.4 Validación del programa de readaptación y reentrenamiento ................ 245

8.5 Programa de entrenamiento (Jiménez-Rubio et al., 2018)....................... 246

8.6 Perfil de expertos (Jiménez-Rubio et al., 2018) ............................................. 250 


\section{Índice de Tablas}

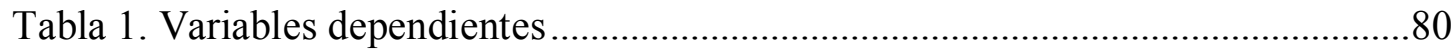

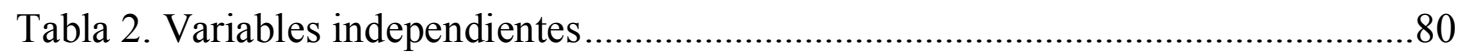

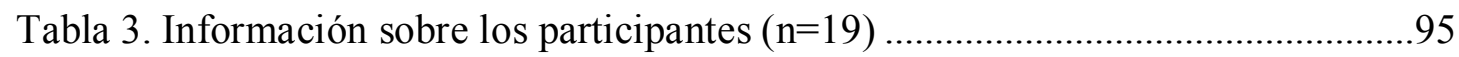

Tabla 4. Desarrollo de los 5 momentos donde se registraron datos .............................99

Tabla 5. Entrenamiento previo a comenzar el programa de readaptación ................. 101

Tabla 6. Modelo de Readaptación en campo para lesiones en la unión miotendinosa proximal del bíceps femoral (Jiménez-Rubio et al., 2018) ............................... 103

Tabla 7. Evaluación de ítems del modelo con V de Aiken ....................................... 112

Tabla 8. Perfil académico y profesional de los expertos (15) que evaluaron modelo

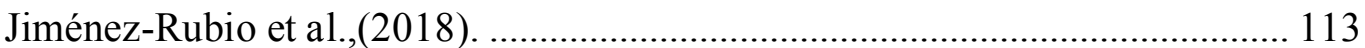

Tabla 9. Descriptivos momentos PRE- REA - GRUPO ............................................ 121

Tabla 10. Comparación PRE-REA-GRUPO para variables de rendimiento relacionadas con la velocidad y WTRR (IC 90\%) ............................................. 122

Tabla 11. Comparación PRE-REA-GRUPO para variables de rendimiento relacionadas con aceleración, desaceleración, sprints, y explosividad (IC 90\%)

Tabla 12. Descriptivos momentos Pre-lesión (PRE) y Competiciones (C1-C2)....... 133

Tabla 13. Comparación PRE-C1-C2 para variables de rendimiento relacionadas con la velocidad y WTRR (IC 90\%)

Tabla 14. Comparación PRE-C1-C2 para variables de rendimiento relacionadas con aceleración, desaceleración, sprints, y explosividad (IC 90\%) 


\section{Índice de Figuras}

Figura 1. Relación entre el número de días de lesión por equipo y la posición final de la liga de los equipos (Arnason et al., 2004) …………........................................... 13

Figura 2. $\mathrm{N}^{\mathrm{o}}$ de lesiones/partido últimos campeonatos del Mundo .................................. 15

Figura 3. Anatomia de la musculatura posterior del muslo (Kapandji, 2010) ............... 18

Figura 4. Fases del ciclo de carrera (Schache et al., 2009, 2010) ..................................... 21

Figura 5. Eficacia del ciclo de sprint con "scissors motion"........................................... 22

Figura 6. Ejemplo de cómo funciona sinérgicamente un músculo biarticular en la

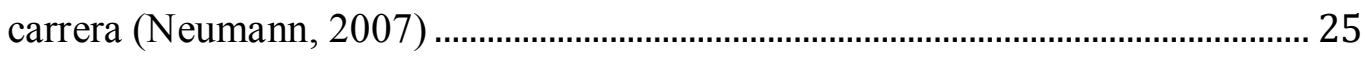

Figura 7. Momento de fuerza de flexores de rodilla (Neumann, 2007)......................... 27

Figura 8. Rango de estiramiento de la unidad musculo-tendinosa de los isquiosurales

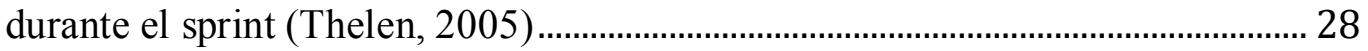

Figura 9 : Unión miotendinosa proximal del bíceps femoral........................................... 29

Figura 10. Parte libre del tendón y septo ó tendón intramuscular................................... 30

Figura 11. RMN de la lesión en la unión miotendinosa proximal del bíceps ............... 31

Figura 12. Áreas de lesión muscular en isquiosurales ...................................................... 32

Figura 13. Fases del golpeo (Orchard 1990) ………........................................................ 35

Figura 14. Patrón de carrera eficaz y eficiente (Bosch \& Ijzerman, 2016)................... 37

Figura 15. Patrón de carrera alterado, que provoca una lesión en unión miotendinosa proximal del bíceps femoral. Fuente: Mediacoach ${ }^{\circledR}$................................................. 37

Figura 16. Etapas para obtención de eficacia en la prevención de lesiones.................... 44

Figura 17. Modelo de prevención de lesiones basado en el entrenamiento con cargas óptimas (Gabbett \& Whiteley, 2017) …………........................................................ 47

Figura 18. Modelo explicativo proceso circular prevención, abordaje lesión y prevención terciaria (post-lesión) (Lalín \& Peirau, 2011)

Figura 19. El Acondicionamiento del deportista es más amplio que la propia lesión

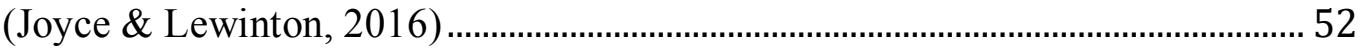

Figura 20. Deportista ejecutando acciones de la intervención con GPS ......................... 81

Figura 21. Chaleco y dispositivo (GPS) para registrar parámetros ................................. 82

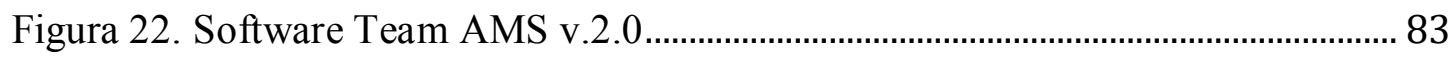

Figura 23. Imagen de registro de sesión/competición con dispositivo GPS ................... 83

Figura 24. Obtención de m/min a V1 (14.4-19.7) ; V2 (19.8-25.1).............................. 84 
Figura 25. Obtención de distancia recorrida, velocidad máxima y velocidad media

$($ Avg_speed)

Figura 26. Obtención del parámetro "work to rest ratio", 86

Figura 27. Obtención del $n^{0}$ de Aceleraciones y Desaceleraciones / min de media intensidad $(2.9-4 \mathrm{~m} / \mathrm{s} 2)$

Figura 28. Obtención del $\mathrm{n}^{\mathrm{o}}$ Aceleraciones y Desaceleraciones /min de alta intensidad $(>4 \mathrm{~m} / \mathrm{s} 2)$ 88

Figura 29. Obtención de número de RSA / min con recuperación $(<6 \mathrm{seg})$ y aceleración

$$
>\text { a } 3 \mathrm{~m} / \mathrm{s} 2
$$

Figura 30. Obtención del número de sprints $/ \mathrm{min}>21 \mathrm{~km} / \mathrm{h}$ 91

Figura 31. Obtención de la aceleración máxima realizada 92

Figura 32. Obtención de número de impactos por encima de $7.1 \mathrm{~g}$ 92

Figura 33. Representación de la muerte experimental 94

Figura 34. Cronología de los momentos donde se registraron datos 99

Figura 35. Diferencias de medias estandarizadas con intervalos de confianza (IC) del 90\% para cada variable relacionada con el rendimiento, entre PRE y REA......129

Figura 36. Diferencias de medias estandarizadas con intervalos de confianza (IC) del 90\% para cada variable relacionada con el rendimiento, entre PRE y GRUPO

Figura 37. Diferencias de medias estandarizadas con intervalos de confianza (IC) del 90\% para cada variable relacionada con el rendimiento, entre REA y GRUPO.

Figura 38. Diferencias de medias estandarizadas con intervalos de confianza (IC) del 90\% para cada variable relacionada con el rendimiento, entre PRE y C1.

Figura 39. Diferencias de medias estandarizadas con intervalos de confianza (IC) del

90\% para cada variable relacionada con el rendimiento, entre PRE y C2 142

Figura 40. Diferencias de medias estandarizadas con intervalos de confianza (IC) del $90 \%$ para cada variable relacionada con el rendimiento, entre $\mathrm{C} 1$ y $\mathrm{C} 2$. 143

Figura 41. Días de vuelta a competición tras lesión (Van der Made et al., 2017) ....150 Figura 42. Valores obtenidos en explosive distance ( $\mathrm{m} / \mathrm{min})$ y en Max_Acc (m/s2) en momentos REA-GRUPO 155

Figura 43. Valores obtenidos en "work to rest ratio" en momentos PRE-REA-GRUPO 
Figura 44. Valores de V1,V2 y V3 (m/min) en momentos PRE-C1-C2 .....................159

Figura 45. Justificación toma de Datos con Getafe CF, validación intervención ......239

Figura 46. Justificación toma de datos con Rayo Vallecano de Madrid, validación

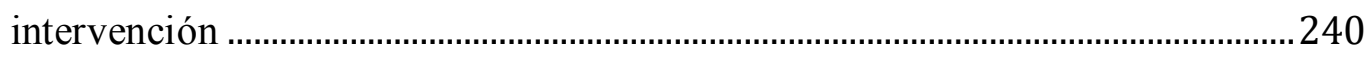

Figura 47. Documento favorable del comité de ética (Datos)..........................................24

Figura 48. Documento favorable del comité de ética (Humanos) .................................242 


\section{Índice de Abreviaturas}

A:C: agudo - crónico

Acc1: $\mathrm{n}^{\mathrm{o}}$ de aceleraciones y desaceleraciones /min entre 2.9 y $4 \mathrm{~m} / \mathrm{s} 2$

Acc2: $\mathrm{n}^{\mathrm{o}}$ de aceleraciones y desaceleraciones/min de más de $4 \mathrm{~m} / \mathrm{s} 2$

AKE: Active knee extension

Avg_speed: Media de velocidad

AVG: Average

BF: Bíceps femoral

$\mathrm{C} 1$ : competición post-lesión (return to play)

C2: 6-10 semanas tras primera competición (medición 5 del estudio)

CCC: Cadena cinética cerrada

CE: Carga externa

CI: Carga interna

COD: Change of direction

CV: Coeficiente de variación

FIFA: Federación Internacional de Fútbol Asociado

GPS: Global positioning system

$\mathrm{H}$ : Hamstrings

HSI: Hamstrings strain injuries

Hz: Herzio

IC: intervalo de confianza

$\mathrm{Km} / \mathrm{h}$ : kilómetros por hora

LPS: Local positioning system

Max_speed: Velocidad máxima

Max_Acc: Aceleración máxima

MRI: Magnetical Resonance Image

OFR: On field rehabilitation

PRE: Momento Pre-lesión (sujeto sano)

PROX: Proximal

Q: Cuádriceps

$\mathrm{RD}$ : Relative distance

REA: Momento Readaptación (momento 2)

RFD: Reeducación funcional deportiva 
RMN: Resonancia Magnética Nuclear

ROM: Range of Motion

RPE: Received perceptive exertion

RSA: Repeated Sprint Ability

RTT: Return to train

RTP: Return to play

SD: Standar desviation

SSG: Small Sided Games

TE: Tamaño del efecto

TRIMP: Training IMPulse

UEFA: Union of European Football Associations

UCJC: Universidad Camilo José Cela

UMT PROX BF: Unión miotendinosa del bíceps femoral a nivel proximal

UMT: Unión miotendinosa

UPM: Universidad Politécnica de Madrid

VO2 max: Consumo máximo de oxígeno

V1: velocidad $1=$ correspondiente a $14.4-19.8 \mathrm{Km} / \mathrm{h}$

V2: velocidad $2=$ correspondiente a $19.8-25.1 \mathrm{Km} / \mathrm{h}$

V3: velocidad $3=$ correspondiente $>25.1 \mathrm{Km} / \mathrm{h}$

WTRR: Work to rest Ratio 


\section{Resumen}

El fútbol profesional es un deporte en cuya práctica existe una alta incidencia de lesión -2 lesiones por jugador y temporada- (Bowen, Gross, Gimpel, Bruce-Low, \& Li, 2019) y especialmente en la musculatura isquiosural. Sin embargo, hasta el momento son escasos los programas de readaptación validados científicamente y apenas existe evidencia sobre los efectos de un programa de readaptación específico para lesiones de la musculatura isquiosural (Taberner \& Cohen, 2018; Taylor, Wright, Dischiavi, Townsend, \& Marmon, 2017).

El presente estudio tuvo dos objetivos principales: 1) diseñar un programa de entrenamiento en fútbol durante el proceso de readaptación y reentrenamiento de la lesión en la unión miotendinosa proximal del bíceps femoral; 2) analizar el efecto de un programa de readaptación en el retorno a la competición post-lesión y en el plazo de las 6-10 semanas siguientes.

Para ello, se llevó a cabo un estudio longitudinal prospectivo cuasi-experimental, en el que participaron 19 futbolistas profesionales que sufrieron la rotura muscular del bíceps femoral en la unión miotendinosa a nivel proximal, y fueron entrenados con un programa de readaptación concreto y específico..

Esta investigación, se realizó con dos equipos de la liga de fútbol profesional masculina en España, y se cuantificó durante 3 temporadas completas (2014-2015 a 2017-2018).

La validación del programa se llevó a cabo a través de un panel de 15 expertos, donde se evaluaron todos los ítems de forma satisfactoria, obteniendo valores en "V" de Aiken entre $0.78-0.98$ (95\% IC : $0.63-0.99)$, superando los valores de 0.75 necesarios para obtener validez (Aiken, 1985; Penfield \& Giacobbi, 2004). 
Mientras que para el análisis del efecto del programa de readaptación, se evaluaron trece variables de condición física de cada sujeto, en cinco momentos diferentes: momento 1 = antes de la lesión; momento 2 = superando completamente el modelo de readaptación; momento 3 = una vez superada la fase de juegos reducidos en el entrenamiento colectivo; momento $4=$ en el regreso a la competición ; momento $5=$ en competición 6 10 semanas posteriores a la primera competición.

Los datos analizados (distancias recorridas, velocidades de desplazamientos, acciones de juego, etc.) fueron recogidos a través de dispositivos "Global Positioning System" (GPS) de $5 \mathrm{~Hz}$ y 80 gr de peso, modelo SPI Elite, (GPSports Systems -Australia-), validados científicamente (Buchheit, Al Haddad, et al., 2014; Gaudino et al., 2013; Lacome et al., 2018).

Los resultados mostraron que los jugadores que habían sufrido una lesión en la unión miotendinosa proximal del bíceps femoral de grado 2 regresaron a la competición en $22.42 \pm 2.32$ días después de someterse a este programa, y no sufrieron una nueva lesión en esa estructura ocho meses después de reaparecer tras dicha lesión.

Respecto al análisis de las variables de condición física específica a lo largo del proceso de readaptación, se observó una mejora progresiva en las variables específicas, especialmente de alta intensidad, a lo largo de los momentos de readaptación y vuelta al entrenamiento y competición.

Se obtuvieron resultados concluyentes en la vuelta a la competición post-lesión en relación a la situación pre-lesión. Se encontraron signos de mejoras, especialmente en valores de velocidad máxima obteniendo diferencias moderadas de 4,68\% ( $p<0,001$; IC $90 \%$ 2,51-6,80\%), en valores de aceleración máxima, donde se obtuvieron diferencias moderadas de 6,66\% ( $\mathrm{p}<0,001$; IC 90\% 3,37-9,83\%). Igualmente en la variable número de impactos superiores a $7.1 \mathrm{~g}$ se obtuvieron diferencias pequeñas de $36,70 \%(p<0,001$; 
IC 90\% 25,33-46,35\%), así como en metros recorridos en velocidad sprint ( $>25,1 \mathrm{~km} / \mathrm{h})$ donde hubo diferencias pequeñas de 31,40\% ( $\mathrm{p}=0,26$; IC 90\% 17,16-43,20\%).

También se obtuvo, al comparar los resultados concernientes al momento de la intervención (momento 2) con la vuelta al entrenamiento en grupo (momento 3) una mejora favorable a momento 3 en la variable $n^{\circ}$ de aceleraciones y desaceleraciones entre $2,9-4 \mathrm{~m} / \mathrm{s} 2 / \mathrm{min}$ con diferencias pequeñas de 40,02\% $(\mathrm{p}=0,001 ; \mathrm{IC} \quad 90 \% 19,57$ $55,27 \%)$. En la variable metros/minuto recorridos en distancia explosiva "variable explosive distance" también se obtuvieron a favor de momento 3, cambios moderados de 12,49\% ( $<<0,001 ;$ IC 90\% 4,42-19,87\%). En la velocidad máxima se obtuvieron cambios moderados de 5,69\% ( $\mathrm{p}=0,029$; IC 90\% 1,11-10,07\%).

Esta mejora en las variables de rendimiento físico fué constatada en el análisis de datos en el momento 5 (fecha de 6-10 semanas después de volver a la competición), donde los resultados fueron favorables respecto al momento de vuelta a la competición (momento 4) obteniendo cambios pequeños de 20,40\% ( $p=0,011$; IC 90\% 6,44-32,29\%), en número de aceleraciones y desaceleraciones $>4 \mathrm{~m} / \mathrm{s} 2 / \mathrm{min}$ así como en metros $/ \mathrm{min}$ recorridos en "explosive distance" también con cambios pequeños de 7,61\% ( $<<0,001$; IC $90 \%$ 4,37-10,75\%).

Los resultados sobre la validación permiten verificar la validez de este programa, así como concluir que dicho programa tiene efectos positivos en el trabajo con futbolistas que sufren una lesión en los isquiosurales para adaptarse a las condiciones reales que demanda la competición. Todo ello, a través de la aplicación de tareas específicas de aceleraciones, empujes, correctas mecánicas de sprint, esfuerzos a alta velocidad y acciones muy similares a los diferentes mecanismos de lesión que actúan como mecanismo protector de la estructura lesionada. 
Por otro lado, la mejora observada en las diferentes variables de rendimiento físico a lo largo de las diferentes fases de aplicación del modelo de readaptación propuesto, confirma la eficacia del presente programa de entrenamiento en la vuelta a la competición así como en la prevención a corto y medio plazo de recidivas.

Palabras clave: Fútbol, Bíceps Femoral, Recuperación, Vuelta a la competición, Prevención 


\section{Abstract}

Professional soccer is a sport in which there is a high incidence of injury, two injuries for player and season (Bowen, Gross, Gimpel, Bruce-Low, \& Li, 2019) and especially in the hamstrings muscles. However, so far scientifically validated rehabilitation programmes are scarce and there is hardly any evidence of the effects of a specific rehabilitation programme for hamstrings strain injuries (Taberner \& Cohen, 2018; Taylor, Wright, Dischiavi, Townsend, \& Marmon, 2017).

The present study had two main objectives: 1) to design a football training program during the process of readaptation and retraining of the injury in the proximal myotendinous junction of the biceps femoris; 2) to analyze the effect of a readaptation program on the return to post-injury competition and within the following 6-10 weeks.

To this end, a quasi-experimental prospective longitudinal study was carried out, in which 19 professional soccer players who suffered a biceps femoris muscle rupture in the proximal miotendinous junction were trained with a specific and concrete readaptation program.

This research was carried out with two teams from the men's professional football league in Spain, and was quantified during 3 complete seasons (2014-2015 to 20172018).

The validation of the program was carried out through a panel of 15 experts, where all the items were evaluated in a satisfactory way, obtaining values in "V" of Aiken between $0.78-0.98(95 \% \mathrm{CI}: 0.63-0.99)$, surpassing the values of 0.75 necessary to obtain validity (Aiken, 1985; Penfield \& Giacobbi, 2004).

While for the analysis of the effect of the rehabilitation program, thirteen physical condition variables of each subject were evaluated at five different moments: moment 1 = before the injury; moment $2=$ completely surpassing the rehabilitation model; 
moment $3=$ once the phase of reduced games in the collective training was surpassed; moment $4=$ in the return to competition; moment $5=$ in competition $6-10$ weeks after the first competition.

The analysed data (distances covered, travel speeds, game actions, etc.) were collected through "Global Positioning System" (GPS) devices of $5 \mathrm{~Hz}$ and 80 gr of weight, model SPI Elite, (GPSports Systems -Australia-), scientifically validated (Buchheit, Al Haddad, et al., 2014; Gaudino et al., 2013; Lacome et al., 2018).

The results showed that players who had suffered an injury to the proximal myotendinous junction of the grade 2 biceps femoris returned to competition in $22.42 \pm 2.32$ days after undergoing this program, and did not suffer a new injury to that structure eight months after reappearing after that injury.

With respect to the analysis of specific physical condition variables throughout the readaptation process, a progressive improvement was observed in the specific variables, especially of high intensity, throughout the moments of readaptation and return to training and competition.

Conclusive results were obtained in the return to competition post-injury in relation to the pre-injury situation. Signs of improvement were found, especially in maximum speed values obtaining moderate differences of $4.68 \%$ ( $<<0.001$; CI 90\% 2.51-6.80\%), in maximum acceleration values, where moderate differences of $6.66 \%(p<0.001$; CI 90\% 3.37-9.83\%) were obtained. Small differences of 36.70\% $(\mathrm{p}<0.001$; CI 90\% 25.33$46.35 \%$ ) were obtained in the variable number of impacts greater than $7.1 \mathrm{~g}$, as well as in meters travelled in sprint speed $(>25.1 \mathrm{~km} / \mathrm{h})$ where there were small differences of $31.40 \%(\mathrm{p}=0.26$; CI 90\% 17.16-43.20\%).

An improvement in the variable no. of accelerations and decelerations between 2.9$4 \mathrm{~m} / \mathrm{s} 2 / \mathrm{min}$ with small differences of $40.02 \%(\mathrm{p}=0.001$; CI $90 \% 19.57-55.27 \%)$ was also 
obtained when comparing the results concerning the time of intervention (time 2) with the return to group training (time 3$)$. Moderate changes of $12.49 \%(\mathrm{p}<0.001 ; \mathrm{CI} 90 \%$ 4.42-19.87\%) were also obtained in the variable meters/minute travelled in explosive distance "variable explosive distance". Moderate changes of 5.69\% ( $p=0.029$; CI 90\% $1.11-10.07 \%$ ) were obtained in the maximum speed.

This improvement in physical performance variables was noted in the analysis of data at time 5 (date 6-10 weeks after returning to competition), where the results were favorable with respect to the time of return to competition (time 4) obtaining small changes of $20.40 \%(\mathrm{p}=0.011$; IC $90 \% 6,44-32,29 \%)$, in number of accelerations and decelerations $>4 \mathrm{~m} / \mathrm{s} 2 / \mathrm{min}$ as well as in meters/min traveled in "explosive distance" also with small changes of $7,61 \%(\mathrm{p}<0,001 ;$ IC $90 \% 4,37-10,75 \%)$.

The results on the validation allow to verify the validity of this program, as well as to conclude that this program has positive effects in the work with footballers who suffer from or are suffering from the same problems.

Keywords: Football, Biceps Femoris, Recovery, Return to Play, Prevention. 
XXXIII 
I.

Marco Teórico 



\subsection{Introducción}

El fútbol de competición actual es una disciplina deportiva con unas altísimas exigencias e importantes demandas (mecánicas, fisiológicas y metabólicas) para el aparato locomotor. En la literatura se muestran estudios donde los futbolistas recorren distancias totales de $11173 \pm 524$ metros (Dellal et al., 2012) llegando a registrar valores de $505 \pm 209$ metros a velocidades por encima de $20,88 \mathrm{~km} / \mathrm{h}$ o distancias que varían entre 600-831 metros a velocidades donde se superan $\operatorname{los} 25,2 \mathrm{~km} / \mathrm{h}$ (Taylor, Wright, Dischiavi, Townsend \& Marmon, 2017). Igualmente son conocidas las exigencias en cuanto a número de aceleraciones totales que se pueden dar en la competición (168 73 en el intervalo de 1,5-2m/s2) (Mallo, Mena, Nevado \& Paredes, 2015).

De acuerdo con varios autores, el fútbol es una actividad catalogada como de alto riesgo de lesión (Fuller et al., 2006; Hägglund, 2007; Van Beijsterveldt, van de Port, Vereijken, \& Backx, 2013) pudiendo tener incluso una incidencia lesiva de hasta mil veces superior si se compara con cualquier otra actividad industrial (Drawer \& Fuller, 2002). En un estudio muy reciente se registra que en el fútbol de alta competición, se producen hasta 2 lesiones por jugador y temporada (Bowen, Gross, Gimpel, Bruce-Low, $\& \mathrm{Li}, 2019)$.

Han sido muchos los estudios publicados, destinados al análisis epidemiológico de las lesiones en el fútbol, debido al alto impacto que este deporte representa a todos los niveles. Sin embargo, es necesario interpretar correctamente los resultados obtenidos, ya que existen discrepancias por la terminología, a pesar de existir algunos documentos de consenso (Fuller et al., 2006; Hamilton et al., 2015; Mueller-Wohlfahrt et al., 2013; Valle et al., 2017).

Cuando un deportista sufre una lesión, tiene como objetivo volver a la competición. El 
proceso que le llevará de retorno al juego y a la competición tiene como objetivo reeducar los movimientos en el contexto real y específico para que el jugador posteriormente se mueva de forma eficaz (Bosch, 2016; Bosch \& Ijzerman, 2016; Van Hooren \& Bosch, 2017).

Durante la lesión, se valoraran patrones que han sido alterados. Este proceso se ha definido como Reeducación Funcional Deportiva y se refiere a todo el proceso que comienza desde la aparición de la lesión hasta el completo retorno al juego (Lalín \& Peirau, 2011). De acuerdo con lo mostrado en la literatura (Caparrós, Pujol, \& Salas, 2017; Lalín \& Peirau, 2011) el proceso de recuperación de una lesión puede dividirse en tres fases: la primera, la fase terapéutica que tiene objetivos eminentemente clínicos; la segunda, una etapa deportiva específica en la que los objetivos son de naturaleza más funcional y se centran en el acondicionamiento físico; y la tercera, que permite al deportista volver a entrenar en las mismas condiciones que el resto de los compañeros no lesionados.

Los profesionales que trabajan con deportistas lesionados, están en el deber de hacerles percibir el momento de la lesión como una oportunidad para el crecimiento, el desarrollo y la mejora de otras cualidades que antes no se entrenaban (Forsdyke, Smith, Jones, \& Gledhill, 2016).

\subsection{Antecedentes y estado de la cuestión}

El enorme impacto del fútbol como disciplina que genera interés en la sociedad se ha visto reflejado a nivel científico, con 9,725 trabajos localizados en Pubmed (fecha de búsqueda: 27/11/2018), aumentando exponencialmente en los últimos años el número de investigaciones en todos los campos de las ciencias del deporte con el objetivo de poder mejorar y evolucionar las estrategias de entrenamiento y preparación de la competición. 
El término de lesión deportiva, se ha definido como daño físico ó incidente que ocurre durante la competición ó sesión de entrenamiento y que obliga al deportista a modificar ó perder una ó más sesiones de entrenamiento y/o a modificar ó abandonar la actividad competitiva (Fuller et al., 2006; Lubetzky-Vilnai, Carmeli, \& Katz-Leurer, 2009).

La problemática sobre la alta incidencia en relación a las lesiones de isquiosurales en el deporte de competición es un hecho evidente (Hägglund, Walden, \& Ekstrand, 2013). Sin embargo, los enfoques hacia la prevención y readaptación de las mismas se han enfocado mayoritariamente hacia propuestas basadas en la evaluación y tratamiento clínico con tareas como nórdico isquiosural "nordic hamstring curls", tareas de flexión de rodilla con fitball con pelvis extendida "Ball leg curl" (Monajati, Larumbe-Zabala, Goss-Sampson, \& Naclerio, 2014), tareas con plataforma de nórdicos "nordboard" (Opar, Piatkowski, Williams, \& Shield, 2013), modelos con tareas del protocolo de Askling “Askling L-Protocol” (Askling, Tengvar, Tarassova, \& Thorstensson, 2014) o pruebas funcionales de extensión activa de la rodilla "AKE test" o de neurodinamia "SLR test" (Neto, Jacobsohn, Carita, \& Oliveira, 2015), así como el test de levantar activamente la pierna "active straight leg raise test" (Liebenson, Karpowicz, Brown, Howarth, \& McGill, 2009), ó tareas de extensión en decúbito prono "Prone hip extension" (Chance-Larsen, Littlewood, \& Garth, 2010). Igualmente con tests de puente glúteo a una pierna "single leg bridge test" (Freckleton, Cook, \& Pizzari, 2014). Otros se han mostrado más enfocados al ejercicio funcional y progresión de tareas de ese perfil. $\mathrm{Y}$ aunque todos ellos muestran resultados respecto a la vuelta a la competición, estos enfoques se expusieron $\sin$ aspectos relacionados con la fase reentrenamiento o rendimiento en fútbol profesional en campo (contexto específico). En esa línea, se conoce el enfoque multifactorial de Mendiguchía y su grupo de investigación “multifactorial approach” (Mendiguchia et al., 2017) u otros más recientes publicados con 
muestra de primer nivel en liga inglesa (Taberner \& Cohen, 2018) que los proponen con tareas en campo, incluso en ese contexto específico y en otras lesiones como las del ligamento cruzado anterior (Verstegen, Falsone, Orr, \& Smith, 2012).

Cabe destacar un estudio publicado muy recientemente que muestra el concepto de recuperación en un contexto real, en este caso con la nomenclatura de recuperación en el campo "on field rehabilitation" (OFR) (Buckthorpe, Frizziero, \& Roi, 2018) demostrando con ello la tendencia hacia un concepto más integral del ejercicio y en un contexto real del entrenamiento como parte importante del proceso de recuperación de este tipo de lesiones.

Gracias a la aportación de dichos estudios, hoy en día se sabe que, desde un punto de vista funcional, los esfuerzos de los jugadores durante la práctica del fútbol se caracterizan por ser intermitentes, compuestos por acciones de corta duración y máxima intensidad como carreras entre 10-20 m y acciones de alta intensidad como saltos, frenadas, recepciones, cambios de dirección o duelos con contacto físico, que se alternan con acciones de baja o moderada intensidad y periodos de recuperación (Casamichana \& Castellano, 2011; Castellano \& Casamichana, 2013; Malone et al., 2016; Rivilla-García et al., 2018; Suárez-Arrones et al., 2015a).

La aparición de la lesión en este contexto de esfuerzos es más fácil de entender dado que se trata de un deporte desarrollado en un entorno inestable, que se ve afectado por variables contextuales o situacionales muy relevantes en el rendimiento competitivo (Castellano, Blanco-Villaseñor, \& Álvarez, 2011; Pratas, Volossovitch, \& Ferreira, 2012).

En los últimos años se han sucedido los estudios que persiguen entender el modo en que dichas variables situacionales afectan al rendimiento deportivo, bien sea desde un punto 
de vista físico, técnico, táctico y/o fisiológico, y especialmente, desde un punto de vista bio-psico-social.

El emergente desarrollo tecnológico de los últimos años, ha provocado la implantación de sistemas de seguimiento semiautomáticos en las mejores ligas europeas, aportando datos sobre posiciones, desplazamientos y velocidades de los jugadores, permitiendo la monitorización y el control de los requerimientos derivados de la competición. De igual forma se están utilizando "local positioning system” y sitemas tracking muy avanzados con diferentes casas comerciales.

La objetividad e inmediatez de estos datos permite tomar decisiones de forma más precisa y acertada así como programar las variables condicionales y preparar los partidos de acuerdo a las exigencias concretas de la competición (Carling, Bloomfield, Nelsen, \& Reilly, 2008).

Es una realidad la carencia de estudios sobre programas de readaptación y a su vez la necesidad recurrente de generar investigaciones acerca de lo ocurrido en los momentos donde los deportistas afrontan una lesión en musculatura isquiosural, y han de minimizar los riesgos para volver a la competición con garantías.

En este sentido, algunos autores han expuesto conceptos importantes en el ámbito de la readaptación y reentrenamiento, mostrando la nomenclatura en referencia a los procesos de readaptación tras haber sufrido una lesión muscular o articular (Caparrós et al., 2017; Lalín \& Peirau, 2011; Pacheco-Arajol \& Pujol Marzo, 2013).

Se reconoce al readaptador físico como un preparador físico que forma y prepara al deportista lesionado (generalmente de forma individual) en la realización de ejercicios apropiados y seguros, y en donde su objetivo es prevenir y restablacer la condición física saludable y/o de rendimiento de éste, además de mejorar su competencia deportiva para 
incorporarse eficaz y eficientemente lo antes posible al entrenamiento de grupo y a la competición tras haber sufrido una lesión (Lalín \& Peirau, 2011).

\subsection{Justificación}

Las lesiones musculares de los isquiosurales, son las más frecuentes en el fútbol profesional y representan alrededor del 12\%-16\% de todas las lesiones en futbolistas de élite (Ekstrand et al., 2012; Hägglund, Walden, \& Ekstrand, 2013; Noya, GómezCarmona, Gracia-Marco, Moliner-Urdiales, \& Sillero-Quintana, 2014).

La rehabilitación y recuperación de las lesiones musculares en isquiosurales tiene como objetivo devolver al jugador a su nivel de rendimiento anterior a la lesión en el menor tiempo posible, minimizando al mismo tiempo el riesgo de volver a lesionarse (Lalín \& Peirau, 2011). Este aspecto es altamente importante ya que la tasa de reincidencia de este tipo de lesiones es alta (16\%-22\%) (Petersen, Thorborg, Nielsen, Budtz-Jørgensen, \& Hölmich, 2011; Pollock et al., 2016) y está relacionada con un tiempo significativamente mayor para el regreso a la competición (Ekstrand, Hagglund, \& Walden, 2011; Van der Made et al., 2017).

Estudios recientes que analizaron datos de alta competición entre las temporadas 2001 y 2014 han mostrado que este tipo de lesiones no ha disminuido en los últimos años sino que ha aumentado entre un 2,3-4\% (Ekstrand, Walden, \& Hägglund, 2016).

De acuerdo a este aumento, algunos autores han indicado que las herramientas preventivas hasta la fecha podrían no ser lo suficientemente eficaces (RomeroRodriguez, 2010). 
Desde este punto de vista, algunos estudios mostraron criterios en cuanto a las tomas de decisiones para la vuelta a la competición de forma segura y clínicamente razonada para evitar la recidiva (Zambaldi, Beasley, \& Rushton, 2017).

La toma de decisiones para la vuelta a la competición de forma segura es un proceso complejo que debe basarse, no solo en los riesgos para la salud y la actividad propia del jugador, sino también en el contexto particular de cada situación: momento de la temporada, nivel competitivo, etc. (Ardern et al., 2016; Creighton, Shrier, Shultz, Meeuwisse, \& Matheson, 2010).

Actualmente no existe consenso definido sobre la evaluación y decisión de cuándo y cómo devolver a la competición a un deportista tras la lesión de la musculatura isquiosural.

Sin embargo, son varios los autores que han referido la necesidad de establecer un consenso para, a partir de datos objetivos, devolver al jugador a la competición tras lesionarse (Ardern et al., 2016; Buckthorpe, Gimpel, Wright, Sturdy, \& Stride, 2017; Mendiguchia \& Brughelli, 2011; Van der Horst, van de Hoef, Reurink, Huisstede, \& Backx, 2016a).

Respecto a la vuelta a la competición tras sufrir una rotura muscular de los isquiosurales, son varios los estudios que registraron el tiempo de recuperación (Hallén \& Ekstrand, 2014a; Stares et al., 2018; Van der Made et al., 2017) donde los datos reportados indicaron una media de $24 \pm 9,7$ días desde que se produce la lesión hasta que el jugador vuelve a competir. Si bien algunos se realizaron con estudiantes (Kilcoyne, Dickens, Keblish, Rue, \& Chronister, 2011).

Si bien son numerosos los criterios que se han utilizado para decidir el momento adecuado para la vuelta a la competición del deportista lesionado, pocos de ellos han 
sido validados científicamente a través de comités de expertos (Van der Horst, Backx, Goedhart, \& Huisstede, 2017; Zambaldi et al., 2017).

Varios autores (Askling, Nilsson, \& Thorstensson, 2010; McCall et al., 2014) definieron la existencia de limitaciones intrínsecas en el fútbol de alto nivel, tales como la ética, la disponibilidad de jugadores, la homogeneidad en el registro y/o la confidencialidad. Aunque en esta línea, destaca el estudio de Noya et al. (2014), en cuánto a recogida de datos en fútbol profesional para concluir epidemiológicamente cómo (sprintando, chutando, girando, decelerando) y cuándo (especialmente al final de cada período) se producían estas lesiones en fútbol profesional.

Numerosos trabajos han referido la necesidad de disponer de criterios objetivos y validados científicamente para volver a la competición de forma segura (Van der Horst, van de Hoef, Reurink, Huisstede, \& Backx, 2016b; Zambaldi et al., 2017) así como modelos de readaptación (Lalín \& Peirau, 2011) que aseguren una vuelta a la competición con garantías de eficacia y, al mismo tiempo, disminuyan al máximo las posiblidades de recidiva para poder mantenerse apto en futuras competiciones.

Sin embargo, actualmente sigue siendo necesario disponer de estos criterios de forma objetiva que hayan sido validados así como modelos de readaptación eficaces para facilitar la gestión del regreso al grupo del deportista y posteriormente a la competición. Por último, es importante referir el elevado coste económico que suponen las lesiones en general, especialmente las lesiones de musculatura isquiosural tanto por su frecuencia como por su recurrencia. Así lo han mostrado varios estudios realizados en fútbol australiano (Hickey, Shield, Williams, \& Opar, 2014). 


\subsection{Delimitación del problema. Epidemiología de lesión .}

Respecto a la localización de las lesiones en fútbol, se ha observado que el muslo es la zona anatómica donde más lesiones se producen (Hägglund, Walden, \& Ekstrand, 2013; Noya et al., 2014).

Vemos como muchas investigaciones revelan la asiduidad con la que los músculos localizados en la parte posterior del muslo, sufren lesiones, considerándose esta estructura anatómica, como la más afectada en el ámbito del deporte profesional (Domínguez-Gasca \& Domínguez-Carrillo, 2011; Ekstrand, Hagglund, et al., 2011) y especialmente en deportes explosivos (Di Salvo et al., 2010; Edouard, Branco, \& Alonso, 2016).

Concretando aún más, se ha constatado un gran porcentaje (67\%) de lesiones en dicha región posterior del muslo (Hawkins, Hulse, Wilkinson, Hodson, \& Gibson, 2001). En la misma línea, Woods et al. (2004) observaron que el 94\% de las lesiones en el muslo eran de tipo muscular. Profundizando aún más en esos registros, el 53\% de estas lesiones se producían en el bíceps femoral, seguido del semitendinoso $16 \%$, semimembranoso $16 \%$ y $\sin$ específicar $19 \%$.

Previamente la literatura mostraba que las lesiones musculares localizadas en la región posterior del muslo representan entre el 12\%-16 de todas las lesiones que se dan en el fútbol profesional (Hägglund, Walden, \& Ekstrand, 2013; Noya et al., 2014). Además, se comparó la incidencia de lesión entre la pierna dominante y la no dominante en esta localización, llegando al resultado de que no había diferencias significativas $(p>0,01)$ entre ello (53\% de las lesiones en la pierna dominante frente a $45 \%$ de las lesiones en la pierna no dominante). 
Otros autores muestran que las lesiones musculares representan cerca de un tercio de todas las lesiones en el fútbol profesional (Ekstrand, Hägglund, \& Walden, 2011) siendo el muslo el lugar anatómico más frecuente para estas lesiones (Ekstrand et al., 2016) y donde vemos que hasta el $53 \%$ de todas las lesiones del muslo se producenen el complejo muscular de los isquiosurales. Dentro de este grupo muscular, el $73 \%$ de los casos afectaron al bíceps femoral (Noya et al., 2014).

En definitiva vemos que las lesiones del complejo isquiotibial representaron el $37 \%$ de todas las lesiones sin contacto (Ekstrand et al., 2016).

De forma más concreta, ciertos trabajos mostraron que el $46 \%$ de las lesiones de los músculos isquiosurales se produce en el área del tendón común del bíceps femoral, es decir, en la unión miotendinosa entre el bíceps y el semitendinoso, en el tercio proximal del muslo (Balius, Pedret, \& Pujol, 2013; Heiderscheit, Sherry, Silder, Chumanov, \& Thelen, 2010).

Otros autores confirman resultados mostrando que alrededor del 25\%-31\% de todas las lesiones en el bíceps femoral se localizan en el tercio proximal de los isquiosurales (Comin, Malliaras, Baquie, Barbour, \& Connell, 2013; DeWitt \& Vidale, 2014)

En cuanto al perfil de esfuerzos, diremos siendo más concretos, la frecuencia con la que la estructura miotendinosa proximal de los isquiosurales se lesiona en deportistas que realizan carreras de máxima velocidad o sprints (Mendiguchia et al., 2016, 2015; Morin et al., 2015).

Como se observa en la figura 1, existe una correlación directa entre número de lesiones y resultado clasificatorio al final de temporada (Arnason et al., 2004; Dalton, Kerr, \& Dompier, 2015; Valle et al., 2017). Por ello, mejorar el registro de menor número de lesiones, podría suponer una mejora en el rendimiento deportivo (Hägglund, Walden, Magnusson, et al., 2013). 


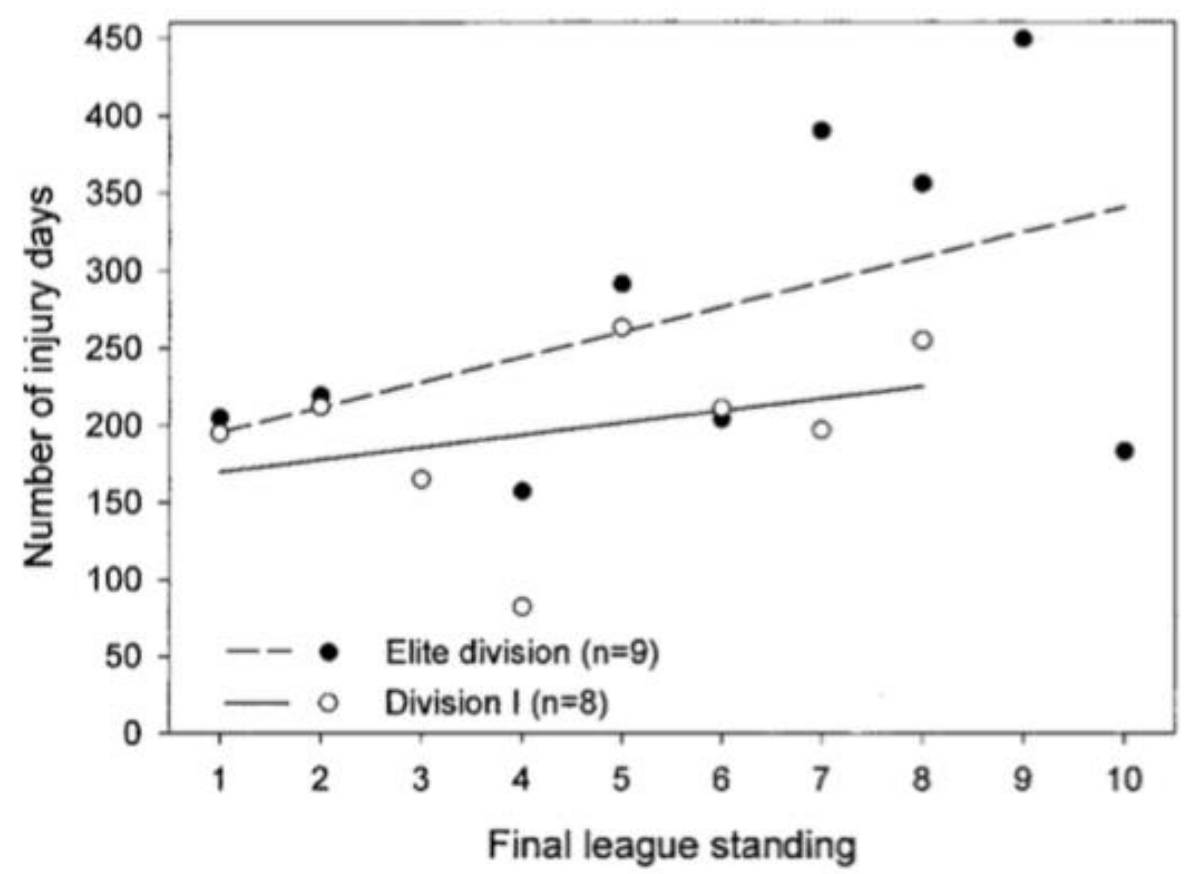

Figura 1. Relación entre el número de días de lesión por equipo y la posición final de la liga de los equipos (Arnason et al., 2004)

En la misma línea, Hägglund et al. (2013) concluyeron que las lesiones tenían una influencia significativa en el rendimiento de la competición liguera y en competiciones europeas en fútbol profesional masculino.

Además de esta relación, el organismo internacional (UEFA) emite anualmente un estudio de la epidemiologia de las lesiones de los clubes europeos en base a unos criterios de clasificación de las lesiones musculares por; severidad, tipo, si son o no por contacto, localización, mecanismos o días de ausencia entre otros, que van en la línea de lo aportado anteriormente.

\subsubsection{Lesiones de isquiosurales en deportistas amateurs/profesionales.}

En relación a lesiones en los isquiosurales vemos que constituyen un porcentaje importante del total de las lesiones músculo-esqueléticas agudas producidas durante actividades deportivas realizadas tanto a nivel profesional como amateur (Price, Hawkins, Hulse, \& Hodson, 2004; Shankar, Fields, Collins, Dick, \& Comstock, 2007). 
De esta forma, diferentes deportistas, tales como corredores (Drezner, 2003) y los participantes en disciplinas deportivas de equipo como el fútbol, el rugby, el baloncesto, el cricket o el fútbol australiano (Askling, Karlsson, \& Thorstensson, 2003; Feeley et al., 2008; Orchard, Kountouris, \& Sims, 2016; Zambaldi et al., 2017) son especialmente propensos a padecer lesiones de este perfil. La prevalencia de dicha lesión reportada en diferentes estudios con modalidades de diversa índole se encuentra entre el 8 y el $25 \%$ (Croisier, 2004; Mason, Dickens, \& Vail, 2012) dependiendo del deporte en cuestión.

\subsubsection{Lesiones de isquiosurales en el fútbol profesional}

El fútbol de alta competición posee una tasa de lesiones entre 3,7 y 29,1 por cada 1000 horas de exposición (entrenamiento y competición) (Ekstrand, Hägglund, et al., 2011; Noya et al., 2014). Como se observa, en la figura 2, se dan entre 0,97-1,67 lesiones por partido (Junge \& Dvorak, 2013), suponiendo ello un mayor número de lesiones ocurridas durante la competición (41,33 lesiones por cada 1000 horas) que en los entrenamientos (6,02 lesiones por cada 1000 horas) (Noya et al., 2014).

En un estudio realizado en las últimas fases finales de copa del mundo grupos de investigación (Junge \& Dvorak, 2013) muestran un valor del 3-17\% de jugadores que sumaron entre 8-28 días de ausencia por temas de lesiones (figura 2). Estudios epidemiológicos recientes han mostrado que el $22 \%$ de los jugadores de una plantilla sufrieron al menos una lesión de los isquiosurales durante una temporada (Ekstrand, Hagglund, et al., 2011) y la tasa de recurrencia varió entre el 8-12\% especialmente en las dos primeras semanas (ver apartado 1.4.3). 


\begin{tabular}{|c|c|c|c|c|c|}
\hline Tournament & France 1998 & Korea/Japan 2002 & Germany 2006 & South Africa 2010 & Brazil 2014 \\
\hline Matches (n) & 64 & 64 & 64 & 64 & 64 \\
\hline Response rate & $124 / 128(97 \%)$ & $128 / 128(100 \%)$ & $128 / 128(100 \%)$ & $124 / 128(97 \%)$ & $124 / 128(97 \%)$ \\
\hline Match hours documented & 2046 & 2112 & 2112 & 2046 & 2046 \\
\hline Injuries ( $n$ ) & 149 & 171 & 145 & 125 & 104 \\
\hline Injuries per 1000 player hours $(95 \% \mathrm{Cl})$ & $72.8(61.1$ to 84.5$)$ & $81.0(68.9$ to 93.1$)$ & 68.7 (57.5 to 79.9 ) & 61.1 (50.4 to 71.8$)$ & $50.8(41.0$ to 60.6$)$ \\
\hline Injuries per match $(95 \% \mathrm{Cl})$ & 2.40 (2.01 to 2.79 ) & 2.67 (2.27 to 3.07$)$ & 2.27 (1.90 to 2.64$)$ & $2.02(1.67$ to 2.37$)$ & $1.68(1.36$ to 2.00$)$ \\
\hline \multicolumn{6}{|c|}{ Estimated duration of absence from sport (days) } \\
\hline 0 & & $53(33 \%)$ & $39(30 \%)$ & $39(35 \%)$ & $41(43 \%)$ \\
\hline $1-3$ & & $59(37 \%)$ & $43(33 \%)$ & $59(49 \%)$ & $15(16 \%)$ \\
\hline $4-7$ & & $27(17 \%)$ & $19(15 \%)$ & $9(7 \%)$ & $15(16 \%)$ \\
\hline $8-28$ & & $18(11 \%)$ & $23(18)$ & $3(3 \%)$ & $16(17 \%)$ \\
\hline 29 and more & & $3(2 \%)$ & $7(5 \%)$ & $2(2 \%)$ & $8(8 \%)$ \\
\hline Not specified & & 0 & 5 & 9 & 6 \\
\hline Missing & & 11 & 9 & 4 & 3 \\
\hline Injuries with subsequent absence & & 107 & 97 & 82 & 60 \\
\hline Injuries per $1000 \mathrm{~h}(95 \% \mathrm{Cl})$ & & 50.7 (41.1 to 60.3 ) & $45.9(36.8$ to 55.0$)$ & 40.1 (31.4 to 48.8$)$ & 29.3 (21.9 to 36.7$)$ \\
\hline Injuries per match $(95 \% \mathrm{Cl})$ & & $1.67(1.35$ to 1.99$)$ & $1.51(1.20$ to 1.80$)$ & $1.29(1.01$ to 1.57$)$ & $0.97(0.72$ to 1.22$)$ \\
\hline
\end{tabular}

Figura 2. $N^{0}$ de lesiones/partido últimos campeonatos del Mundo (Junge \& Dvořák, 2015)

En promedio, cuando un jugador sufre una lesión de los músculos isquiosurales, el jugador se ausenta de entrenamientos normalizados con grupo alrededor de 18 días y 3 3.5 partidos (Woods et al., 2004).

Otros trabajos mostraron resultados vuelta a la competición diferenciando en deportistas que habían sufrido lesión en tendón intramuscular (figura 10) mostrando un valor de 22,2 $\pm 7,4$ días (Van der Made et al., 2017)

\subsubsection{Recidivas y consecuencias de las lesiones de isquiosurales.}

Se conoce como recidiva a la lesión del mismo tipo y misma localización que una anterior y que sucede después del regreso de un jugador a la participación total desde la lesión inicial (Fuller et al., 2006; Lempainen et al., 2015). En el mismo trabajo se cita que si esta recaída sucede en los dos meses posteriores al regreso a la participación total se denominará "recidiva temprana", entre los 2 y los 12 meses posteriores al regreso será "recidiva tardía", y "recidiva retrasada" si ocurre después de los 12 meses del regreso a la actividad total. 
Varios estudios (Malliaropoulos et al., 2015, 2010; Orchard \& Seward, 2002) mostraron un porcentaje importante, de hasta el $12 \%$, de posibles recidivas en la musculatura isquiosural dentro de las 2 semanas posteriores a la vuelta a la competición. Otros estudios cuantificaron en un $13 \%$ las recidivas durante la primera semana y un $8 \%$ durante la segunda semana (Orchard \& Seward, 2004).

Además de la alta incidencia de lesión y del alto riesgo de recaer en una lesión de este tipo, vemos trabajos que afirman que la tasa de recidiva dentro de los 2 primeros meses es del 22\% (Petersen et al., 2011) o en otros estudios más actuales parecen mostrar una reducción de este índice de recidiva a un 8.6\% (Van der Made et al., 2017).

Por tanto, es constatado que la recaída en este tipo de lesiones parece manifestarse con asiduidad, suponiendo para el fútbol un importante problema (Brukner, Nealon, Morgan, Burgess, \& Dunn, 2014) ya que implica habitualmente una mayor gravedad, un mayor número de días de ausencia así como numerosas consecuencias de diversa índole: pérdida de rendimiento del deportista lesionado y otras (Ekstrand, Hagglund, et al., 2011; Hägglund, Walden, Magnusson, et al., 2013).

Son numerosas las causas descritas sobre esta incidencia, algunas investigaciones hicieron referencia a factores como insuficientes programas de rehabilitaciónreadaptación y/o vuelta prematura a la realización de actividad deportiva de alta exigencia (Caparrós et al., 2017; Elliott, Zarins, Powell, \& Kenyon, 2011; Mendiguchia \& Brughelli, 2011), otras investigaciones lo asocian a las altas demandas del juego, y el corto espacio de tiempo entre competiciones (Taylor et al., 2017).

Dada la importancia y frecuencia de las recidivas en las lesiones de isquiosurales en fútbol profesional, son varias las propuestas y programas que se han implementado con 
el objetivo de evitarlas (Fort-Vanmeerhaeghe, Romero-Rodriguez, Lloyd, Kushner, \& Myer, 2016; Fort-Vanmeerhaeghe \& Romero, 2013; Romero-Rodriguez, 2010).

Algunos autores, con el objetivo de minimizar las recidivas intentan correlacionar pruebas radiológicas con momentos para volver al deporte (Svensson, Alricsson, Eckerman, Magounakis, \& Werner, 2016). Aunque ellos muestran que aproximadamente un tercio de las elongaciones en musculatura isquiosural recurrirán.

\subsection{Grupo muscular isquiosural: características y función}

Existe un amplio espectro de lesiones relacionados con la musculatura isquiosural que incluyen elongaciones, avulsiones proximales completas y parciales del tendón común isquiosural, avulsiones apofisarias isquiáticas, tendinopatías a nivel proximal del tendón común isquiosural y dolor en la parte posterior del muslo referido (Hamilton et al., 2015; Sherry, Johnston, \& Heiderscheit, 2015; Valle et al., 2017)

De entre todas, las elongaciones son las lesiones más prevalentes en muchas disciplinas deportivas (Askling, Saartok, \& Thorstensson, 2006).

\subsubsection{Ubicación}

En la parte posterior del muslo, se pueden distinguir tres músculos, que forman el complejo muscular de los isquio-tibio-peroneos: bíceps femoral, semitendinoso y semimembranoso.

El bíceps femoral se encuentra en ese compartimento posterior del muslo y junto al semimembranoso y semitendinoso se les conoce como músculos "isquiosurales". Todos excepto la cabeza corta del bíceps femoral cruzan la articulación de la cadera y de la rodilla (Kapandji, 2010).

El bíceps femoral tiene dos cabezas: 
- La cabeza larga se origina junto al músculo semitendinoso de la parte inferomedial del área superior de la tuberosidad isquiática.

- La cabeza corta surge del lado lateral de la línea áspera en la diáfisis del fémur.

El vientre muscular de la cabeza larga cruza la porción posterior del muslo de forma oblicua de medial a lateral y se une a la cabeza corta a nivel distal. Juntas, las fibras de las dos cabeza forman un tendón, que es palpable en la cara lateral de la porción distal del muslo. La parte principal del tendón se inserta en la superficie lateral de la cabeza del peroné y la extensiones del tendón se fusionan con el ligamento colateral peroneo y con ligamentos asociados a la cara lateral de la articulación de la rodilla (Kapandji, 2010).

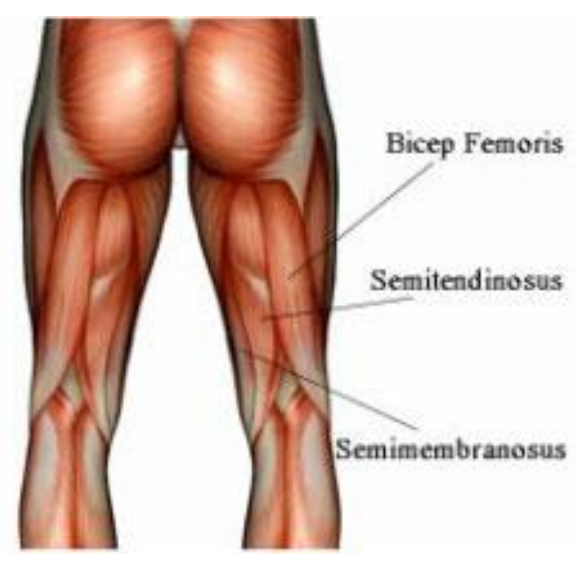

Figura 3. Anatomia de la musculatura posterior del muslo (Kapandji, 2010)

\subsubsection{Función}

Como grupo, los isquiosurales son músculos biarticulares ya que flexionan la articulación de la rodilla y extienden la articulación de la cadera, además de ser rotadores de ambas articulaciones (Kapandji, 2010).

Su acción en la rodilla está condicionada por la posición de la cadera, ya que cuando 
ésta se flexiona la distancia que separa las inserciones de estos músculos aumenta regularmente. De este modo, cuanto más se flexiona la cadera, mayor es el acortamiento relativo de los isquiosurales y más se tensan (Kapandji, 2010).

También está condicionada por la posición de la rodilla, ya que su eficacia en la cadera depende en gran medida de la posición de la rodilla, puesto que el bloqueo de la rodilla en extensión favorece su acción extensora de la cadera, existiendo por lo tanto una relación de antagonismo-sinergismo entre los isquiosurales y el cuádriceps (Kapandji, 2010).

Los isquiosurales tienen una función esencial en la estabilización de la cadera, puesto que cuando la pelvis bascula hacia delante el centro de gravedad pasa por delante de la línea de las caderas, siendo los isquiosurales los primeros en iniciar la acción para estabilizarla (Kapandji, 2010).

Esta musculatura también cumple una función esencial en el patrón de la marcha o la locomoción cuando los isquiotibiles realizan la extensión y el glúteo mayor no interviene. No ocurre lo mismo al correr, saltar o caminar cuesta arriba, donde el glúteo mayor no solo es indispensable sino que su función desempeña un papel principal, especialmente en la triple extension (Schuermans, Danneels, Van Tiggelen, Palmans, \& Witvrouw, 2017).

\subsubsection{Cinemática de la carrera en relación a los isquiosurales.}

Analizar y conocer la cinemática biarticular de los isquiosurales en la cadera y la rodilla es importante ya que permite identificar las causas que pueden provocar mecanismos de tensión a nivel muscular y tendinoso (Bosch \& Ijzerman, 2016; Chumanov, Schache, 
Heiderscheit, \& Thelen, 2012; Neumann, Rowan, \& González del Campo Román, 2007).

La carrera de máxima velocidad o sprint es el movimiento más estudiado en la literatura en relación al mecanismo de lesión de los isquiosurales (ver figuras 14-15) (Bosch \& Ijzerman, 2016; Higashihara, Nagano, Ono, \& Fukubayashi, 2018; Morin et al., 2015; Rumpf, Lockie, Cronin, \& Jalilvand, 2016) y, por ello, es necesario abordar y analizar la artrocinemática en el plano sagital (Taylor, Wright, Dischiavi, Townsend, \& Marmon, 2017) para estudiar cómo funciona y cómo se activa el bíceps femoral desde una perspectiva biomecánica real a la acción del gesto explosivo del sprint, en su relación con el tronco y con el apoyo distal, el pié (Brigaud, 2016).

En el sprint se produce un movimiento cíclico, oscilatorio y explosivo de flexión de cadera y extensión de rodilla activa y en cadena cinética abierta en el cual la articulación coxofemoral gira sobre el fémur y en la articulación femorotibial es la tibia la que se desliza y rueda sobre el fémur, produciendo una tensión en la musculatura isquiotibial, y en muchas ocasiones en situaciones de incertidumbre (Brigaud, 2016; Morin et al., 2015).

En el fútbol profesional, diferentes autores consideran que el deportista se ha puesto en sprint cuando supera velocidades de $18 \mathrm{~km} / \mathrm{h}$ (Casamichana, Castellano, \& Castagna, 2012) pero el parámetro estandarizado por sistemas tracking de medición, evalúa el sprint a partir de $21 \mathrm{~km} / \mathrm{h}$ (Suárez-Arrones et al., 2015b).

Con objeto de explicar lo que ocurre en el sprint, se ha dicho que, los isquiosurales están prácticamente siempre activos, contrayéndose en acortamiento para tirar de la pierna hacia atrás a través de la postura y actuando excéntricamente para ralentizar y controlar el movimiento hacia adelante de la extremidad distal al final de la fase de vuelo 
(“swing”) (Chumanov, Heiderscheit, \& Thelen, 2011; Thelen, Chumanov, Hoerth, et al., 2005).

Aunque el complejo muscular en su conjunto es activo tanto en la fase concéntrica como en la excéntrica, la cinemática se produce a través del ciclo estiramiento acortamiento (CEA), donde la mayor aportación para el empuje y aceleración proviene del bíceps femoral (Chumanov, Heiderscheit, \& Thelen, 2007; Chumanov, Wille, Michalski, \& Heiderscheit, 2012). Se cree que el riesgo de lesión de los isquiosurales relacionado con sprint se limita predominantemente al estrés de cabeza larga del bíceps femoral (Askling, Malliaropoulos, \& Karlsson, 2012).

En un análisis actual del alto rendimiento en fútbol se han observado también lesiones en el músculo semitendinoso durante picos de aceleración (Erickson \& Sherry, 2017a).

Desde este punto de vista, una línea creciente de investigación apunta a la fase final de vuelo de la carrera como el momento en el que es probable que el bíceps femoral se lesione (Heiderscheit et al., 2005; Schache, Kim, Morgan, \& Pandy, 2010; Schache, Wrigley, Baker, \& Pandy, 2009) (Figura 4).

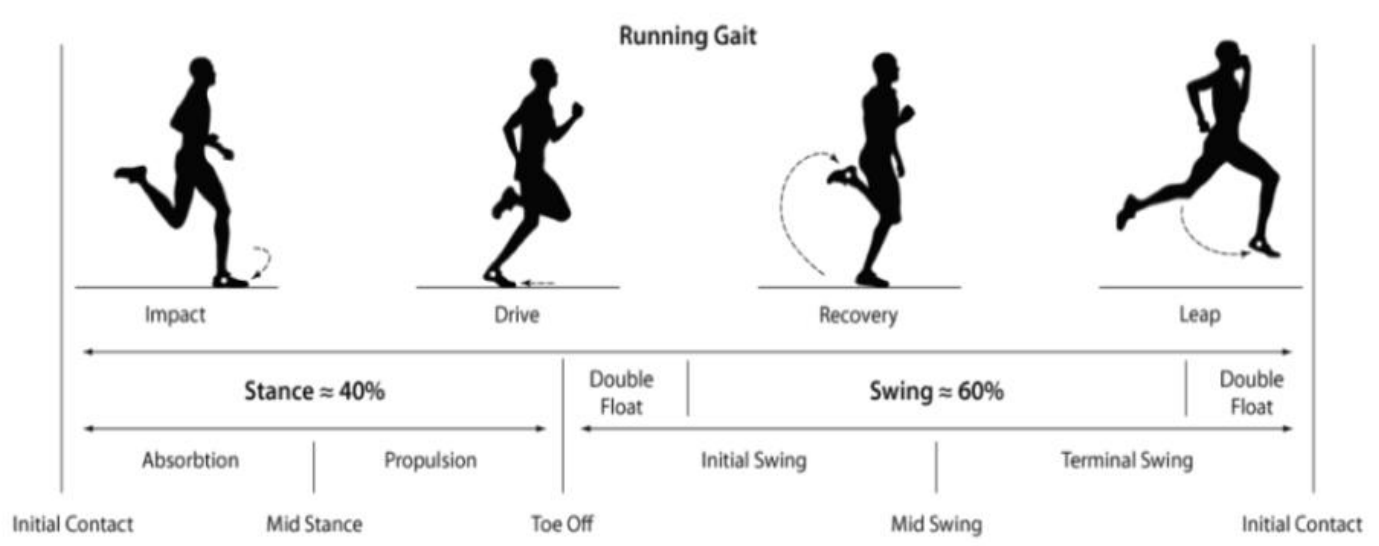

Figura 4. Fases del ciclo de carrera (Schache et al., 2009, 2010) 
Durante esta fase de vuelo, la cadera se flexiona y la rodilla se acerca a la extensión para que el pié contacte con el suelo. En ese momento, el bíceps femoral se está alargando y está actuando excéntricamente para disminuir la velocidad de la tibia en preparación para el contacto del pié. En este punto (cerca de la extensión total de la rodilla), el brazo de momento del bíceps femoral está disminuyendo rápidamente y el músculo está perdiendo ventaja biomecánica (Brigaud, 2016; Herzog \& Read, 1993). En esta línea de investigación, también encontramos trabajos de Bosch e Ijzerman (2016) donde se ha desglosado la importancia de la eficacia en el sprint, mostrando la necesidad de entrenar el concepto "cadera en movimiento tijera" "scissors motion" (figura 5) con objeto de ser estímulo preventivo y evitar así lesiones de este tipo al incluir activaciones protectoras de la zona lumboabominal (Shield \& Bourne, 2018).

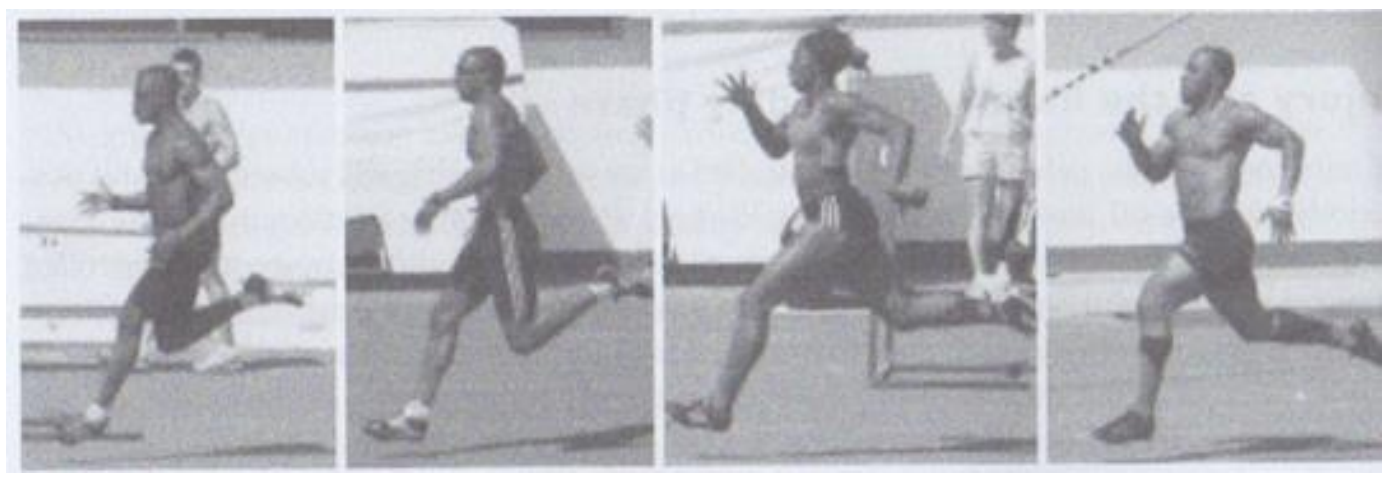

Figura 5. Eficacia del ciclo de sprint con "scissors motion".

(Bosch \& Ijzerman, 2016).

En un sprint, la producción máxima de fuerza por los músculos flexores de la rodilla suele tener lugar cerca de la extensión completa. Aunque los isquiosurales tengan su brazo de palanca máximo con la rodilla flexionada a 45 grados (figura 7), estos músculos producen el mayor momento flexor de rodilla cuando están elongados por completo, ya que la relación magnitud-tensión parece ser un factor que influye mucho en 
la magnitud del momento flexor potencial de los isquiosurales (Neumann et al., 2007).

Así, el riesgo de lesiones radica en la combinación incontrolada de un grupo muscular tensionado que genera elevadas fuerzas excéntricas.

En cuanto a las velocidades de contracción, Chumanov et al. (2007) examinaron las diferencias entre la velocidad submáxima (80\%) y la velocidad máxima de sprint, encontrando que aunque el estiramiento máximo del isquiosural no cambió de forma significativa, la fuerza máxima neta de dicho isquiosural aumentó de $36 \mathrm{~N} / \mathrm{kg}$ a $52 \mathrm{~N} / \mathrm{kg}$ (es decir, un aumento del 38\%), y el trabajo negativo neto promedio aumentó de 1,4 a 2,6 J/kg (es decir, un aumento del 86\%).

Todo ello implica un importante conocimiento para elaborar futuros programas de reentrenamiento cuando programemos tareas que estén en el $80 \%$ de la velocidad máxima.

El gran aumento de la energía por unidad de masa (es decir, la carga de trabajo excéntrica) entre el funcionamiento submáximo y el máximo apuntala uno de los mecanismos de lesión del isquiosural a alta velocidad. Cuando esta carga de trabajo excede los niveles máximos de tolerancia, la estructura muscular sufre daños agudos (Chumanov et al., 2011; Heiderscheit et al., 2005; Schache et al., 2010; Thelen, Chumanov, Hoerth, et al., 2005; Thelen, Chumanov, Sherry, \& Heiderscheit, 2006).

En la metodología de esta tesis doctoral, hemos buscado no subestimar las características biomecánicas de los deportistas después de una lesión asociada a la carrera a alta velocidad que afecta típicamente a la unión musculotendinosa proximal del bíceps femoral.

Askling et al. (2006) definieron en un estudio con deportistas de danza, un tipo de lesión alternativa denominada "estiramiento lento" que ocurre en el momento final del rango 
del isquiosural cuando la cadera es flexionada y la rodilla es forzada a la extensión próximo al contacto del pié, aunque en este caso, no en el sprint. Esta lesión involucra habitualmente al semimembranoso a nivel proximal y se asocia con un período de rehabilitación más prolongado (Askling, Tengvar, Saartok, \& Thorstensson, 2008).

Por otro lado, las lesiones de isquiosurales en el fútbol no se dan exclusivamente en sprints sino también situaciones donde aparecen elementos perturbantes externos al juego (estado del terreno, condiciones meteorológicas) o intrínsecos (contacto del rival, velocidad y posición del balón etc). Es lo que Bosch \& Ijzerman (2016) denominan las "open skills" o habilidades abiertas.

En relación a la cadera como punto de origen del bíceps femoral en la extensión coxofemoral de la pelvis sobre el fémur, el soporte muscular de la postura en inclinación es sobre todo responsabilidad de los músculos isquiosurales, puesto que durante una anteflexión la fuerza del peso del cuerpo se desplaza justo anterior al eje transversal de rotación de la cadera, siendo la cadera restringida por la activación de los músculos isquiosurales y glúteo mayor. Sin embargo, a mayor anteflexión del tronco, el glúteo mayor se mantiene relativamente inactivo y aumenta la activación de los isquiosurales (Busquet, 2009).

Este aparente aumento de la responsabilidad de los isquiosurales, en comparación con el glúteo mayor puede explicarse biomecánicamente a que una inclinación anterior aumenta el brazo de palanca de momento extensor de los isquiosurales, al tiempo que reduce el brazo de palanca del momento extensor de cadera del glúteo mayor (Neumann et al., 2007).

En la figura 6, puede observarse un ejemplo de la relación glúteo e isquiosurales en el patron de aceleración. Ésta ha sido una de las razones más importantes de incluir en el 
programa previo al césped (Tabla 5) el entrenamiento del glúteo mayor en cadena cinética cerrada, especialmente absorbiendo y trasmitiendo fuerzas para desarrollar desplazamientos eficientes.

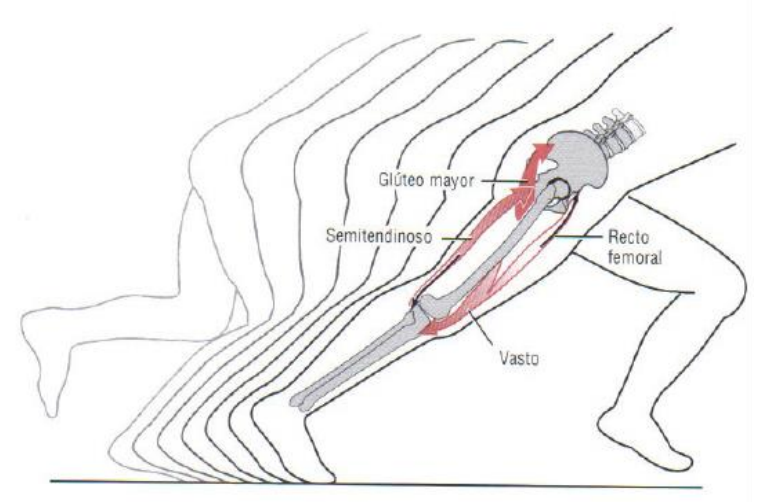

Figura 6. Ejemplo de cómo funciona sinérgicamente un músculo biarticular en la carrera (Neumann, 2007)

La inclinación anterior del tronco, como es en el caso de la carrera, optimiza mecánicamente el momento extensor potencial de los isquiosurales (ver figura 14). Una inclinación anterior significativa elongar los músculos isquiosurales en las articulaciones coxofemoral y femorotibial (Kapandji, 2010).

En relación a la rodilla durante la extensión de la tibia sobre el fémur en la fase de carrera, la superficie articular de la tibia rueda y se desliza en sentido anterior sobre los cóndilos femorales gracias a la acción del cuádriceps, mientras que los isquiosurales ejercen la acción contraria de frenar la oscilación de la tibia.

Estas acciones se producen gracias a las sinergias entre los músculos biarticulares de la cadera y de la rodilla, de los cuales muchos movimientos realizados comprenden acciones cíclicas de extensión de cadera y la rodilla o la flexión de la cadera y la rodilla. Estos patrones de movimiento son componentes fundamentales de la marcha o la carrera 
por ejemplo, ya que le extensión de la cadera y la rodilla impulsan el cuerpo hacia delante o arriba, mientras que la flexión de la cadera y la rodilla adelantan o hacen balancear la extremidad inferior (ver figura 14). Igualmente, es importante entender el perfil de acciones propias del fútbol, donde se incluye un elevado componente de incertidumbre y acciones en entornos abiertos e incontrolados (Bosch \& Ijzerman, 2016).

Pero, ¿cómo se sincronizan estos músculos biarticulares? Para entender mejor estas sinergias como es el caso del bíceps femoral, debemos acudir a la "paradoja de Lombard", ya parece que tenemos una lucha en cada articulación con resultados diferentes.

Siguiendo a Martín (2016), si nos centramos en la cadera, la lucha entre ambas musculaturas sería ganada por la musculatura posterior. Caso contrario ocurre en la rodilla, donde la musculatura anterior es la ganadora. Pero, ¿cómo ocurre esto?. La respuesta la encontramos en los brazos de momento que tiene cada músculo en cada articulación.

Picerno (2017) encontró que el brazo de momento del recto anterior sobre la articulación de la cadera es menor $(3,9 \mathrm{~cm})$ que el de la musculatura posterior $(6,7 \mathrm{~cm})$ al encontrarse su inserción más cercana al eje de rotación. Mientras que en la rodilla ocurrirá lo contrario, el brazo de momento de la musculatura anterior será mayor $(4,4 \mathrm{~cm})$ al encontrarse más alejado del eje de rotación de la rodilla que la musculatura posterior (3,4 cm). Siendo así, la musculatura con mayor brazo de momento en cada articulación será la que marque el movimiento que se realice en esa articulación.

En relación a las fuerza aplicadas generadoras de potencia de los isquiosurales, estas son producidas relativamente bajas o moderadas pero con una velocidad relativamente alta 
de acortamiento o elongación.
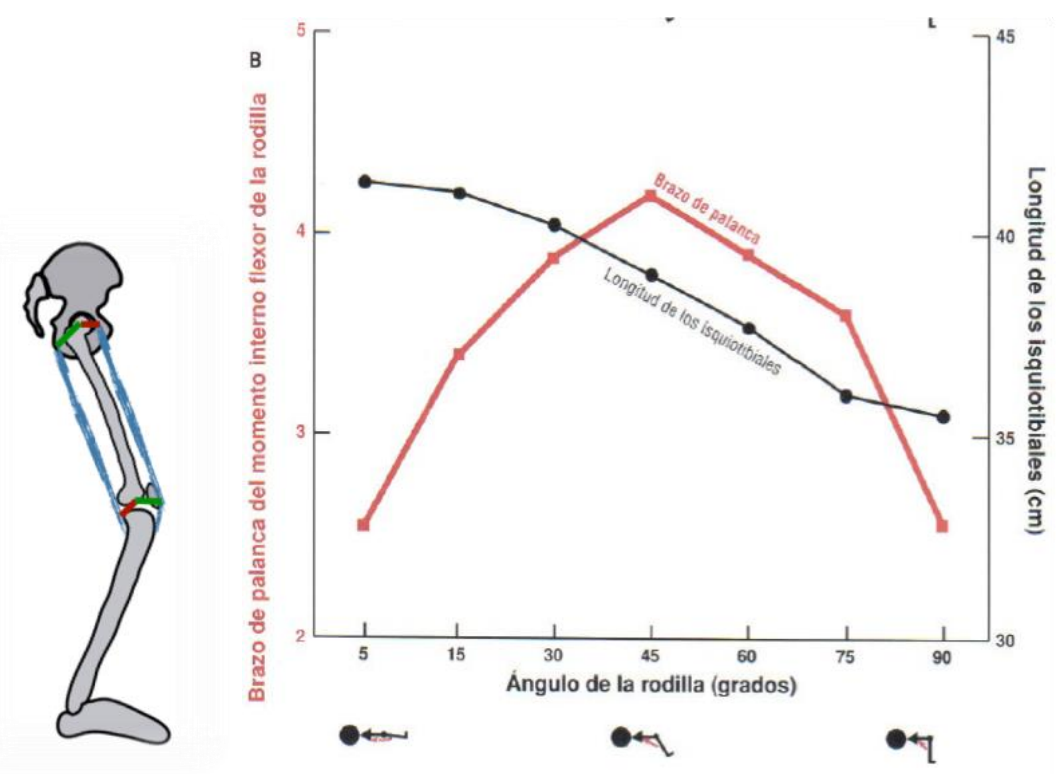

Figura 7. Momento de fuerza de flexores de rodilla (Neumann, 2007)

Thelen et al. (2005) también comprobaron esta paradoja, y analizaron la musculatura isquiotibial durante un sprint, donde encontraron que el tendón del bíceps femoral sufría mayor tensión durante los 45-90 grados de la fase de vuelo, mientras que el pico de longitud máximo de la musculatura isquiosural se producía a finales de la fase oscilatoria cuando la cadera está flexionada 55-65 grados y la rodilla 30-45 grados.

Logicamente, ello supone salir de la normalidad de tensión (figura 8) en los tres componentes de la musculatura isquiosural y sobrepasar tensiones que en ocasiones puedan ser causantes de lesiones (Thelen, Chumanov, Best, Swanson, \& Heiderscheit, 2005), especialmente si no hay adaptaciones previas correctas. 


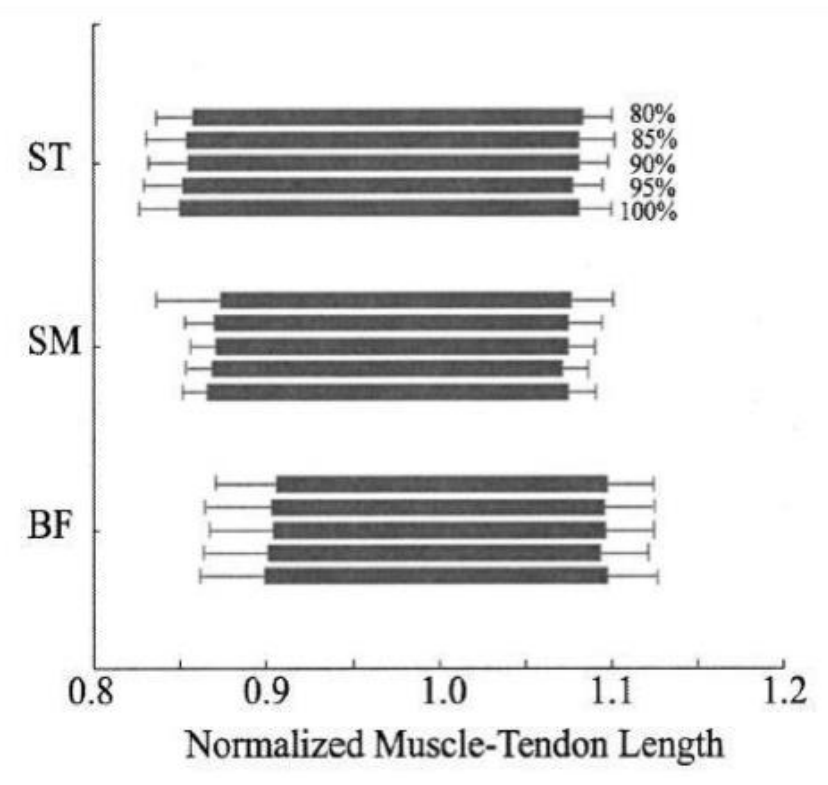

Figura 8. Rango de estiramiento de la unidad musculo-tendinosa de los isquiosurales durante el sprint (Thelen, 2005)

\subsubsection{Localización de la lesión isquiosural}

No existe consenso en la clasificación en cuanto a las localizaciones de lesión en isquiosurales, aunque estudios muy actuales (Valle et al., 2017, 2018) muestran propuestas en cuanto a la nomenclatura, con siglas según si es por traumatismo o mecanismo indirecto, según los mecanismos lesionales, localizaciones concretas (proximal, distal o tercio medio), severidad de la lesión (grados 0-3 definidos) y si es o no una recidiva.

Nomenclatura que en un futuro tenderá a ser estándar con objeto de una mejor comunicación entre profesionales para informar del tipo de lesión.

En cuanto a la frecuencia y localización, la literatura científica refleja al bíceps femoral $\mathrm{y}$, concretamente, a su porción larga, como el lugar donde habitualmente se localiza el daño muscular (Balius, Pedret, Blasi Brugué, et al., 2013; Koulouris \& Connell, 2003).

Más concretamente, la localización más frecuente de esta lesión se encuentra en la unión miotendinosa proximal del bíceps femoral (figura 9) (Balius, Pedret, \& Pujol, 2013; 
Heiderscheit et al., 2010), afectando en un 11\% de ocasiones al tendón intramuscular (figura 9) (Van der Made et al., 2017).

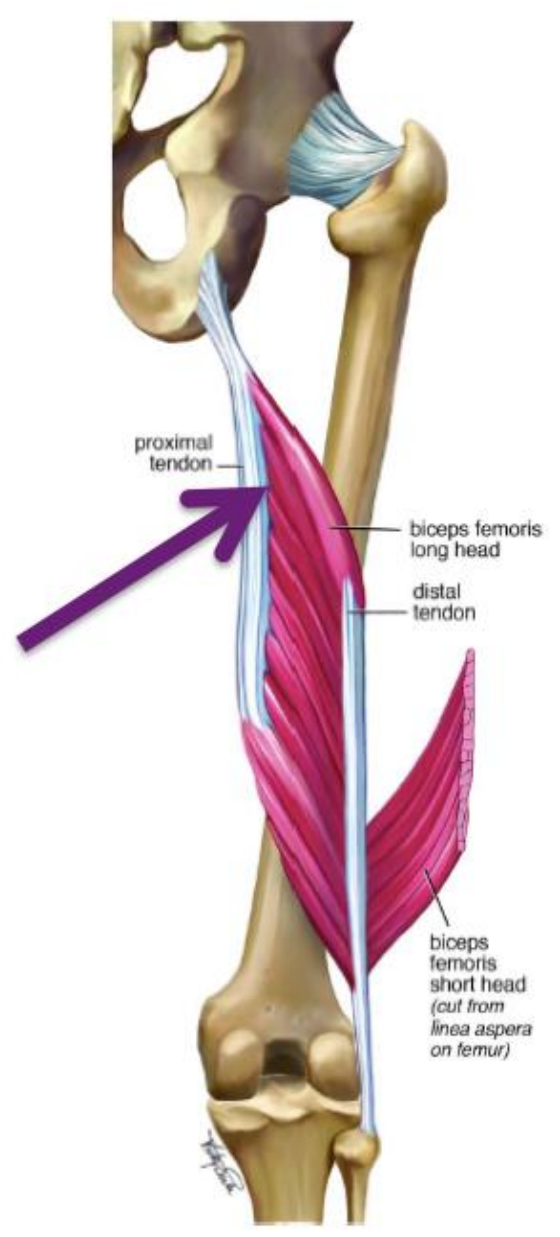

Figura 9 : Unión miotendinosa proximal del bíceps femoral (Brukner \& Connell, 2015) 


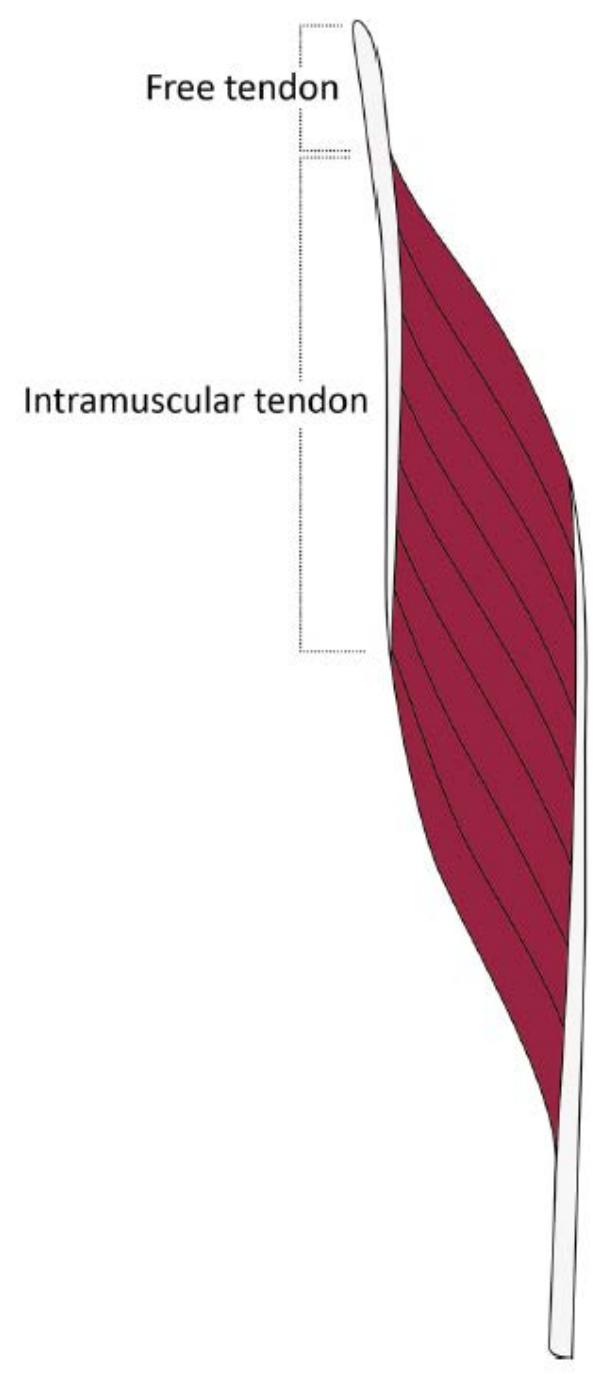

Figura 10. Parte libre del tendón y septo ó tendón intramuscular (Van der Made et al., 2017)

En el momento de producción de la lesión, la ética profesional indica que debe ser diagnosticada por el médico con una exploración clínica y confirmada con pruebas complementarias radiológicas como magnética nuclear (figrua 11) y/o ecografía musculo esquelética) (Hickey, Timmins, Maniar, Williams, \& Opar, 2017).

Los enfoques diagnósticos actuales se basan en gran medida en estudios e imágenes, en particular la resonancia magnética (figura 11). Sin embargo, los hallazgos de la RMN están mal correlacionados con las tasas de nuevas lesiones (De Vos et al., 2014) y los 
atletas a menudo vuelven a lesionarse en ausencia de evidencia radiológica de lesiones persistentes ó anormalidad estructural.

A través de la exploración de características más detalladas, los indicadores de riesgo de volver a lesionarse pueden ser identificables. Esta información puede dar a conocer mas detalles del proceso de rehabilitación y ser utilizada para desarrollar programas de detección y tratamiento, así como pruebas de diagnóstico para guiar la rehabilitación e informar sobre el retorno a la toma de decisiones deportivas.

En referencia a la localización, también hay estudios en referencia a musculatura isquiosural con propuestas de diferentes activaciones con electromiografía bien en región proximal y/o distal basados principalmente en ejercicios analíticos (Hegyi, Csala, Péter, Finni, \& Cronin, 2019; Schoenfeld et al., 2015; Tsaklis et al., 2015; Zebis et al., 2013), sin embargo, también leemos estudios basados más en el movimiento también con electromiografia (Higashihara, Nagano, Ono, \& Fukubayashi, 2015; Higashihara et al., 2018).

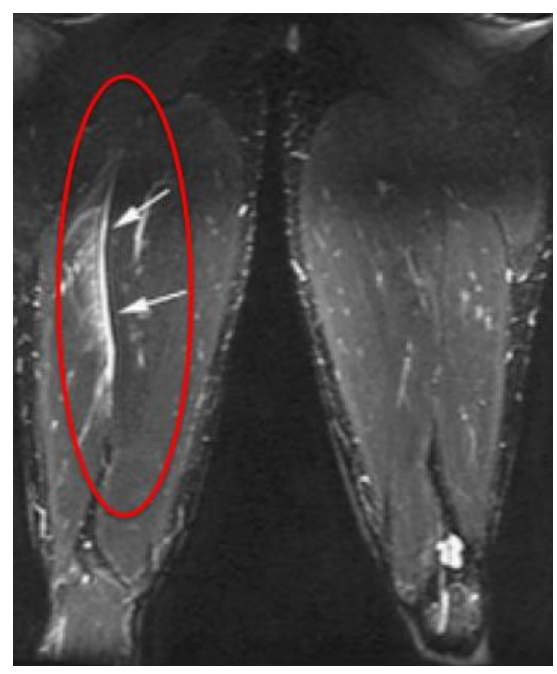

Figura 11. RMN de la lesión en la unión miotendinosa proximal del bíceps femoral (Armfield, Kim, Towers, Bradley, \& Robertson, 2006) 
Por otra parte, se ha relacionado el pronóstico de la lesión con el área, la longitud y la proximidad de la lesión a la tuberosidad isquiática. En cambio, las lesiones proximales del músculo en el semimembranoso, se producen por una situación de hiperestiramiento y afectan la zona tendinosa de éste, lo que condiciona, en ocasiones, una vuelta a la competición más larga, y hasta el momento, muy poco estudiada (Balius, Pedret, \& Pujol, 2013).

Otros autores confirman resultados mostrando que alrededor del 25\%-31\% de todas las lesiones en el bíceps femoral se localizan en el tercio proximal de los isquiosurales (Comin, Malliaras, Baquie, Barbour, \& Connell, 2013; DeWitt \& Vidale, 2014). Otros autores, mostraron mayor precisión en cuanto al tejido lesionado (Chan, Del Buono, Best, \& Maffulli, 2012; Järvinen \& Kalimo, 2013) diferenciando entre 5 tipos o perfiles de lesión muscular en isquiosurales (intramuscular, miofascial, miofascial/perifascial, miotendinosa y combinadas) (figura 12).

Table 2 Proposed classification system

Site of lesion

1. Proximal MTJ

2. Muscle

A. Proximal a. Intramuscular

B. Middle

b. Myofascial

C. Distal

c. Myofascial/perifascial

d. Myotendinous

e. Combined

3. Distal MTJ

$M T J$ musculo-tendinous junction

Figura 12. Áreas de lesión muscular en isquiosurales

(Chan et al., 2012; Järvinen \& Kalimo, 2013) 


\subsubsection{Causas de lesión. Mecanismos lesionales}

Una de las causas más determinantes que la literatura nos muestra en relación a la asiduidad de la lesión en bíceps femoral, es su doble inervación entre porción corta y porción larga (Clark, 2008) y ello ha de tenerse en cuenta a la hora de implementar criterios de readaptación en lesiones del bíceps femoral.

En los 2 útlimos años, han aparecido más publicaciones en referencia a cómo y por dónde se lesionan los isquiosurales en deportes explosivos, como consecuencia de necesidad de encontrar mecanismos preventivos (Méndez-Villanueva et al., 2016; Mendiguchia et al., 2017, 2015). En algunos de ellos, no solamente se ha medido a través de ejercicios y con electromiografía de superficie, sino evaluando la mecánica del sprint (Higashihara et al., 2018) y los cambios de dirección con incertidumbre en el juego (Dos'Santos, Thomas, Comfort, \& A. Jones, 2018).

Por otra parte, como se describió anteriormente, es importante saber que la gran mayoría de las lesiones producidas en la musculatura isquiosural, se producen durante acciones de carrera a alta velocidad (Arnason, Andersen, Holme, Engebretsen, \& Bahr, 2008; Woods et al., 2004). Según Woods et al. (2004), tan solo un 7\% de las lesiones son producidas por contacto, mientras que el $91 \%$ restante se deben a mecanismos lesionales indirectos. Aún así estudios muy actuales, continúan reservando un apartado en las clasificaciones a las lesiones por traumatismo directo en relación a los isquiosurales (Valle et al., 2018).

La literatura previa muestra que la mayoría de los mecanismos de lesión en los isquiosurales fueron clasificados como no-contacto (Hagglund, Walden, \& Ekstrand, 2006; Hawkins et al., 2001; Opar, Williams, \& Shield, 2012a; Schmitt, Tim, \& McHugh, 2012; Woods et al., 2004). 
En otro orden de interpretación en cuanto a las causas y mecanismos, se ha mostrado que en el fútbol de alto nivel existen una gran cantidad de acciones explosivas como son las aceleraciones, desaceleraciones y cambios de dirección a máxima velocidad (Goldman \& Jones, 2011; Van Beijsterveldt et al., 2013), y con ello, las probabilidades de sufrir una lesión en la musculatura isquiosural son altas.

\subsubsection{El sprint como mecanismo de lesión}

Factores biomecánicos tienen una relación muy directa con el sprint y la lesión. Concretamente, una mecánica alterada muy común en deportistas es correr en retroversión pélvica, flexión de la columna lumbar y entrada de retropié. Ello conlleva una mayor tensión en el bíceps femoral durante la fase final de vuelo en la carrera (Bosch \& Ijzerman, 2016; Brigaud, 2016; Thelen et al., 2006).

En otro orden, la tardía activación de musculatura estabilizadora y profunda lumbar (multífidos) así como una pobre activación del psoas ilíaco, incrementa posibilidades para una ineficaz mecánica en el sprint (Raabe \& Chaudhari, 2018).

Este grupo muscular, los isquiosurales, tiene la capacidad de producir grandes fuerzas (Garrett, Califf, \& H Bassett, 1984), lo que tiene una importante repercusión en aquellas situaciones deportivas que impliquen aceleraciones, acciones a alta velocidad y cambios de dirección.

El mecanismo del sprint es el que justifica en gran medida la prevalencia de esta lesión en isquiosurales en deportes que suponen actividades explosivas, aceleraciones, desaceleraciones, y rápidos cambios de dirección ó saltos (Drezner, 2003; Morin et al., 2015). 
El mayor estiramiento músculo-tendinoso se produce sobre el bíceps femoral (Thelen, Chumanov, Best, et al., 2005), lo que puede contribuir a que éste sea el músculo que mayor tendencia tiene a lesionarse (Askling, Tengvar, Saartok, \& Thorstensson, 2007).

Por otro lado, cuando la lesión se produce por un sobreestiramiento, hay estudios que parecen incluir como la localización más común el tendón proximal del semimembranoso (Askling et al., 2006, 2008), pero hay otros autores que también hacen referencia al bíceps femoral como el más frecuente en este mecanismo de lesión concretamente en la porción larga del bíceps femoral (Askling et al., 2014).

\subsubsection{El golpeo como mecanismo de lesión}

Chutar es una de las habilidades más importantes en el fútbol. Una buena técnica de golpeo es importante para mejorar parámetros de rendimiento y con ello ser determinante en el juego, siempre a través de hacer goles.

Disponer de una buena técnica de golpeo se basa en una óptima coordinación entre las extremidades (Nunome, IkegamI, Kozakai, Apriantono, \& Sano, 2006) y en la transferencia inter-segmental de energía. Estas acciones aumentan así las posibilidades de marcar un gol (Kellis, Katis, \& Gissis, 2004). Por esta razón, la mejora de la técnica en golpeo (figura 13) con el empeine de fútbol es uno de los objetivos más importantes de los programas de entrenamiento.

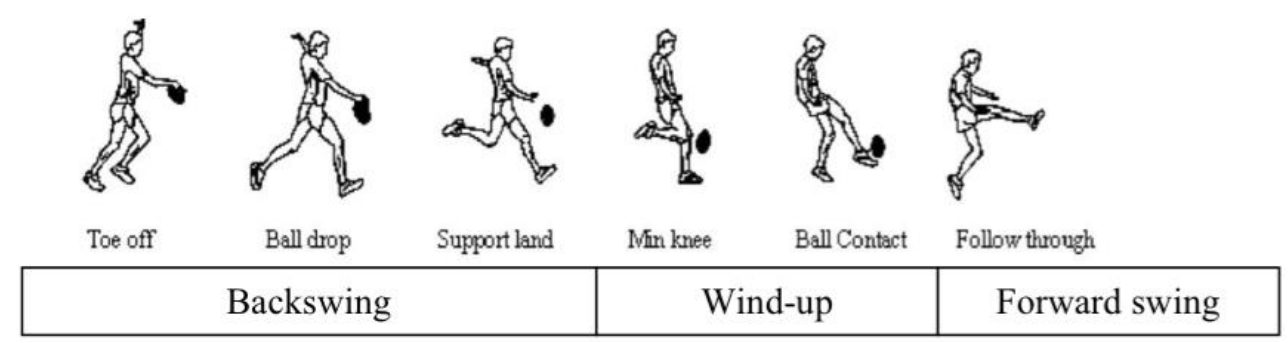

Figura 13. Fases del golpeo (Orchard 1990) 
Aunque el mecanismo de lesión más frecuente en la musculatura isquiosural es el sprint, los isquiosurales también pueden sufrir daño estructural durante el golpeo (Crossley, Zhang, Schache, Bryant, \& Cowan, 2011). Sin embargo, el número de estudios en esta línea es limitado revelando que existen diferencias en este tipo de lesión en función del género (Navandar et al., 2015; Navandar, Gulino, Antonio, \& Navarro, 2013).

Esta lesión por distensión/elongación de los isquiosurales tiene una alta tasa de reincidencia (Wangensteen et al., 2016) lo cual podría ser explicado a través de un análisis de la técnica en el golpeo a través de un enfoque dinámico inverso.

Woods et al. (2004) encontraron que correr y disparar fueron la primera y la tercera causa más común de una lesión de isquiosurales sin contacto.

\subsubsection{Otros mecanismos de lesión}

Otros mecanismos lesionales menos citados en literatura, debido a su menor frecuencia, son los que se producen en las habilidades abiertas (figuras 14-15) como hacer un despeje o tratar de controlar un balón en carrera, con descontrol de zona central y en solicitación excéntrica, cuando el tronco regresa al centro de masas. Es en ese momento donde pueden romper los isquiosurales (Higashihara, Ono, Kubota, Okuwaki, \& Fukubayashi, 2010). 


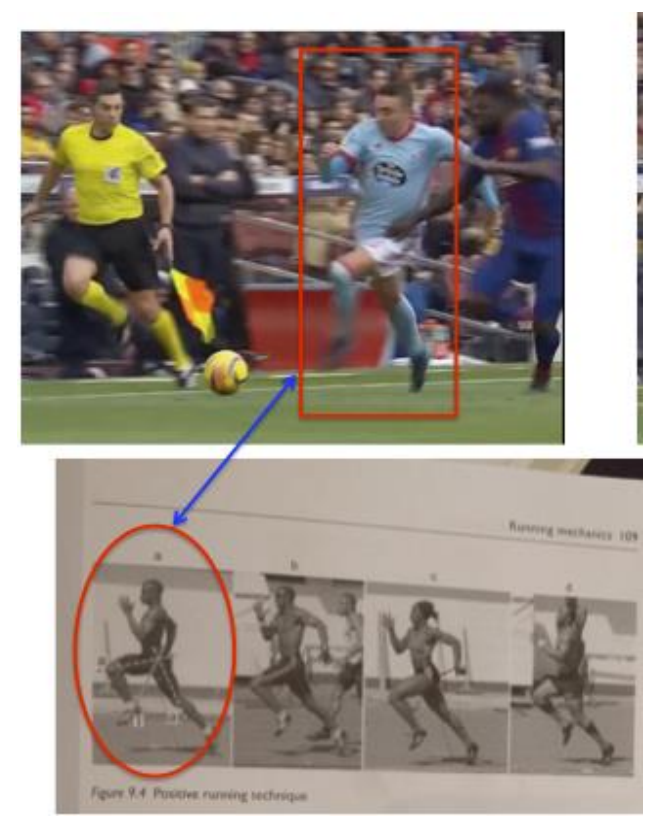

Figura 14. Patrón de carrera eficaz y eficiente (Bosch \& Ijzerman, 2016)

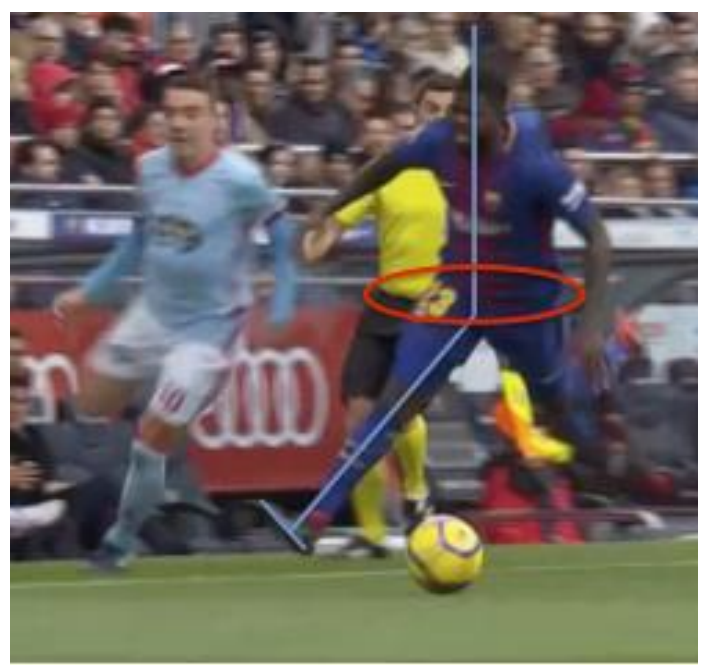

Figura 15. Patrón de carrera alterado, que provoca una lesión en unión miotendinosa proximal del bíceps femoral. Fuente: Mediacoach ${ }^{\circledR}$ 


\subsection{Estrategias preventivas}

Durante los últimos años, han aparecido propuestas de prevención como la de Monajati et al. (2016) en lesiones del ligamento cruzado anterior y en lesiones de isquiosurales, en las cuales se incluía profilaxis a través de aterrizajes, incluso no planeados, y a través de cambios de dirección con incertidumbre, que vemos en la práctica real que son situaciones no favorecedoras para la salud de los isquiosurales, especialmente en situaciones de descontrol, en tareas abiertas (Bosch \& Ijzerman, 2016).

El tipo de acciones que habitualmente ocasionan u originan la lesión isquiosural entre otras, podrían estar protegidas, si en esas mismas acciones el deportista es capaz de generar fuerza contra el suelo y realizar así con mayor garantía otra acción posterior a ésta (Brigaud, 2016). En este sentido, la prevención pasa por la necesidad de reeducar la aceleración, la desaceleración y reaceleración en los patrones deportivos (Bloomfield, Polman, \& O’Donoghue, 2007; Wehbe, Hartwig, \& Duncan, 2014b), así como dar valor al entrenamiento del componente extensor del glúteo mayor, en el empuje contra el suelo en la triple extensión (Alcaraz, Carlos-Vivas, Oponjuru, \& Martínez-Rodríguez, 2018; Bosch, 2016; Morin et al., 2015; Schuermans, Van Tiggelen, \& Witvrouw, 2017).

\subsubsection{Factores de riesgo}

La naturaleza multifacética de la lesión de los isquiosurales, ha llevado a que se enumeren numerosos factores de riesgo, incluso a dudar si se está yendo en la dirección correcta (Mendiguchia, Alentorn-Geli, \& Brughelli, 2012).

Cada vez se reconoce más que los factores de riesgo en las lesiones de los músculos isquiosurales son complejos y multifactoriales (Mendiguchia et al., 2012).

Estos factores de riesgo se han clasificado de la siguiente forma: 


\section{No modificables:}

- Edad (Arnason et al., 2004; Edouard, Branco, \& Alonso, 2016; Verrall, Slavotinek, Barnes, Fon, \& Spriggins, 2001).

- Lesión previa (Bourne et al., 2016; Hagglund et al., 2006; Timmins, 2017).

- Predisposición genética (Pickering \& Kiely, 2018).

\section{Modificables:}

- Fuerza muscular (Croisier, Ganteaume, Binet, Genty, \& Ferret, 2008; Opar et al., 2015; Sugiura, Sakuma, Sakuraba, \& Sato, 2017).

- Fatiga neuromuscular / Sobreuso (Ekstrand et al., 2012; Opar et al., 2012a; Schuermans, Danneels, et al., 2017; Woods et al., 2004).

- Descoordinacción en patrones de movimiento que involucran al bíceps femoral (Bourne et al., 2017; Timmins, 2017).

- Flexibilidad (Bradley, Olsen, \& Portas, 2007; G. Henderson, Barnes, \& Portas, 2010; Witvrouw, Danneels, Asselman, D’Have, \& Cambier, 2003).

- Flexión lumbar incontrolada / inhibición multífidos (Castillo \& Lieberman, 2015; Raabe \& Chaudhari, 2018).

- Déficit de actividad del psoas y déficits rango de movimiento en coxofemoral (Castillo \& Lieberman, 2015; Raabe \& Chaudhari, 2018).

- No estabilidad o déficit de control en zona central (Shield \& Bourne, 2018).

Identificar y considerar cada uno de estos factores de riesgo debería ayudar a desarrollar perfiles de riesgo individuales en el intento de reducir las lesiones de isquiosurales así como su recidiva.

En este sentido, es muy destacable el hecho de que un historial previo de lesión isquiosural suponga ser el principal factor de riesgo y se asocia con un aumento de 2 a 6 
veces en el riesgo (De Visser, Reijman, Heijboer, \& Bos, 2012; Mendiguchia et al., 2012).

Concretamente Ekstrand et al. (2012) constataron un aumento de 11,6 veces en el riesgo de volver a lesionarse en el fútbol de élite en cuanto a la asociación durante la temporada posterior a una lesión isquiosural, mientras que Visser et al. (2012) registraron tasas de reincidencia entre el $12-63 \%$ en diferentes disciplinas deportivas, evaluando tanto en una temporada como en 2 temporadas subsiguientes.

El elevado número de factores de riesgo, así como la complejidad de los mecanismos que provocan las lesiones en los isquiosurales, supone un gran desafío para los staffs médicos y técnicos de clubes de alto rendimiento.

El incremento de la edad parece ser el factor de riesgo intrínseco más destacado, mostrando diversos estudios con deportistas una relación estadísticamente significativa (Gabbe, Bennell, Finch, Wajswelner, \& Orchard, 2006; Opar, Williams, \& Shield, 2012b).

De esta forma, se ha constatado que los deportistas mayores de 23 años presentan una incidencia de 1,3 a 3,9 veces más riesgo, mientras que en los mayores de 25 años la incidencia asciende hasta 2,8 a 4,4 veces más (De Hoyo et al., 2016). Los resultados dan lugar a la interpretación de que el riesgo de lesión aumenta un 30\% con cada año de vida deportiva (Engebretsen, Myklebust, Holme, Engebretsen, \& Bahr, 2010; Verrall et al., 2001).

Las razones propuestas incluyen reducciones naturales en el volumen muscular relacionadas con la edad, pero parece menos probable que esto se aplique a los deportistas de élite de 24 años de edad. Otra posible explicación está en la posible relación entre la disfunción de los músculos isquiosurales y los cambios degenerativos 
lumbares (Hallén \& Ekstrand, 2014b; Mueller-Wohlfahrt et al., 2013). Quizás la compresión de la raíz del nervio o el control postural inadaptado ligado a la disfunción espinal podría tener una relación con la función de los isquiosurales.

Silder et al. (2008) informaron de la existencia de una proliferación de tejido cicatricial en la región de la unión musculotendinosa después de una lesión de isquiosurales, lo que provoca un aumento significativo de los niveles de tensión en todo el músculo, especialmente en las fibras musculares adyacentes durante las contracciones excéntricas. Si estos niveles de tensión superan los límites mecánicos de estas fibras, es probable que se produzcan nuevas lesiones (Järvinen, Kääriäinen, Äärimaa, Vaittinen, \& Kalimo, 2007; Järvinen, Kääriäinen, \& Kalimo, 2005).

Para minimizar este posible factor de riesgo - la restricción provocada por tejido cicatricial- parecen ser claves las intervenciones enfocadas a evitar restricciones entre planos miofasciales (Valera-Garrido, Minaya-Muñoz, \& Medina-Mirapeix, 2014) y la movilización temprana y controlada una vez aparece la lesión (Järvinen, 1977; Järvinen et al., 2007).

En esta línea, con carácter profiláctico, novedosas técnicas terapéuticas ecoguiadas, parecen ser una interesante propuesta.

La rehabilitación temprana y efectiva después de la lesión puede ser clave para minimizar la formación excesiva de cicatrices. El nivel de fuerza, y más concretamente la asimetría de fuerza, es decir, la descompensación entre niveles desarrollados por cuádriceps y por isquiosurales, tiene una asociación significativa con la lesión de isquiosurales, según valores de tests isocinéticos (Croisier et al., 2008). 
Sin embargo, algunos estudios no hallaron esta asociación entre la asimetría de fuerza entre cuádriceps e isquiosurales y el riesgo de lesión (Freckleton \& Pizzari, 2013; Proske, Morgan, Brockett, \& Percival, 2004), asociando el problema a otros factores.

La fatiga así como el estrés propio de la competición parecen, igualmente, factores de riesgo en este tipo de lesiones. Lo cual podría explicar el hecho de que las lesiones de isquiosurales hayan tendido a producirse al final de cada mitad de los partidos de fútbol europeos, especialmente en el segundo periodo (Woods et al., 2004).

Algunos autores, han vinculado esta asociación con la intensidad de la competición

(Bengtsson, Ekstrand, Walden, \& Hägglund, 2017; Ekstrand, Hägglund, et al., 2011).

Ambos factores sugieren que la fatiga y las lesiones están asociadas. Esto puede estar relacionado con consecuencias físiológicas dentro de los músculos o efectos más sistémicos.

Algunas causas que explican mayor número de lesiones en bíceps femoral respecto al grupo de isquiosurales internos, como semitendinoso-semimembranoso apuntan a descoordinaciones en cuanto a la inervación dada la diferente inervación entre porción larga y corta del bíceps femoral (Clark, 2008).

\subsubsection{Modelos de prevención}

De acuerdo con lo expuesto anteriormente, siendo el fútbol catalogado como un deporte de un alto riesgo de lesión y teniendo en cuenta que es el bíceps femoral el músculo que más lesiones sufre con esta práctica deportiva, se considera de gran importancia destinar los esfuerzos hacia la prevención de dicha lesión (Bengtsson, Ekstrand, \& Hägglund, 2013; Ekstrand et al., 2012; Woods et al., 2004) con el objetivo de disminuir el riesgo lesivo e impactar positivamente en los diferentes factores de influencia (Prior, Guerin, \& Grimmer, 2009). 
Las situaciones lesivas requieren de la coordinación de un equipo multidisciplinar de profesionales, con el objetivo de prevenir, recuperar, readaptar y reentrenar al deportista hacia las exigencias que demanda el entrenamiento y la competición de manera óptima, y siempre que fuese posible, mejorando el retorno temprano (Prior et al., 2009).

Las actuaciones orientadas a la prevención de lesiones, a pesar de haber mostrado elevada eficacia (Casais, 2008; Cruz-Ferreira, Marujo, Folgado, Gutierres Filho, \& Fernandes, 2015) no se han implementado de manera sistemática en muchas modalidades deportivas. Es por ello, que la prevención de lesiones supone un reto importante dentro del contexto seleccionado, con el fin de compartir conocimientos, y colaborar en la reducción de procesos lesionales que perturben el buen funcionamiento de la práctica deportiva.

Van Mechelen (1992) propuso cuatro pasos para evaluar las medidas preventivas que permitirían evitar una determinada lesión. Dicha secuencia se exponía de la siguiente manera: 1. Conocer la amplitud del problema; 2. Identificar los factores y mecanismos lesionales; 3. Introducir las medidas de prevención; 4. Evaluación de su eficacia (Van Mechelen, Hlobil, \& Kemper, 1992). Como puede observarse en la figura 16, algunos estudios (Romero Rodrígez \& Tous Fajardo, 2010) y otros más recientes (Padua et al., 2014) han ampliado y detallado este proceso. 


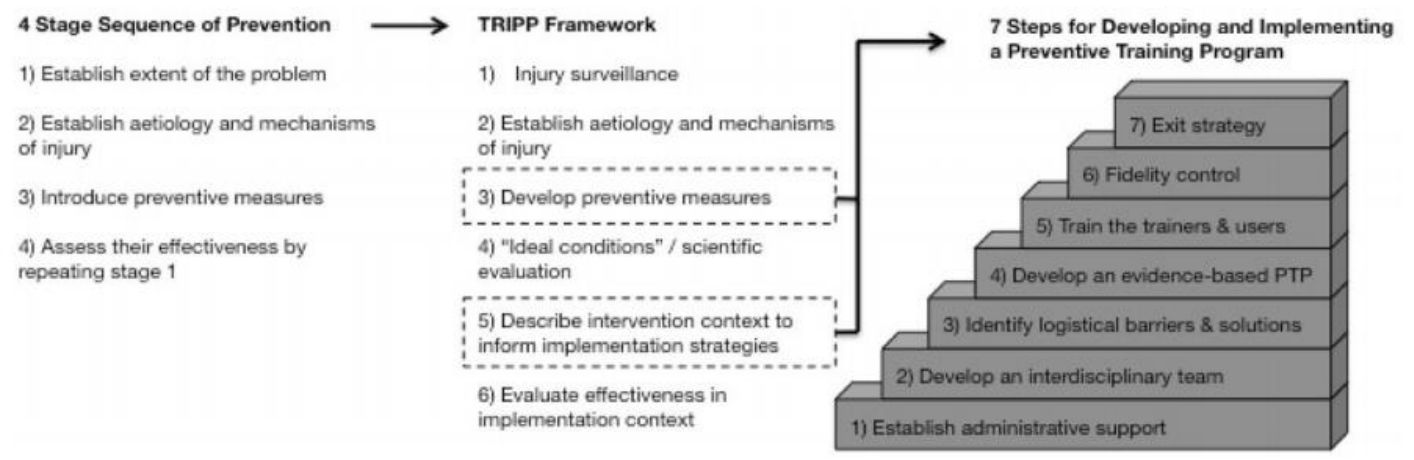

Figura 16. Etapas para obtención de eficacia en la prevención de lesiones

(Padua et al., 2014)

Por otro lado, Romero-Rodríguez \& Tous-Fajardo (2010) han establecido que los pilares neuromusculares en los que debe sustentarse un plan de prevención de lesiones en el deporte son los siguientes: (Romero Rodrígez \& Tous Fajardo, 2010)

- Fuerza: es la cualidad física por excelencia en la prevención de lesiones. Es necesario planificarla de forma correcta, teniendo en cuenta los diferentes tipos de contracción (concéntrica, excéntrica e isométrica).

- Sistema propioceptivo- visual- vestibular: se considera necesario realizar un trabajo propioceptivo a través de perturbaciones que lograrán estimular de forma óptima los receptores propioceptivos, mejorando así las cualidades de equilibrio y capacidad de reacción.

- Coordinación neuromuscular: capacidad que permite el control y regulación del cuerpo en la realización de un movimiento.

Teniendo en cuenta que en deportes como el fútbol, donde predominan las habilidades abiertas y de incertidumbre, existen sistemas complejos de control del participante sobre el juego, consideramos adecuado que exista un enfoque integral en el abordaje de la 
prevención desde el punto de vista de reducción del riesgo de lesión (Barrett et al., 2016). Por ello, diremos que no todos los modelos preventivos son lineales, en la actividad física y el deporte y dichos sistemas complejos están presentes en los procesos de adaptación, aprendizaje y entrenamiento del deportista.

Sería deseable que dichos sistemas funcionaran como una unidad y su comportamiento se caracterizase por el control situacional, permitiendo así su eficacia y eficiencia en contextos imprevisibles, como en la aparición de las lesiones, en situaciones de incertidumbre (Bosch, 2016).

En este sentido es interesante mencionar la importancia del trabajo neuromuscular en relación a la prevención de lesiones, y qué autores publican en la actualidad (Maeo, Shan, Otsuka, Kanehisa, \& Kawakami, 2018).

Algunos autores (Luisa \& Francisco, 2004) han explicado la compleja y frecuente aparición de la lesión de isquiosurales a través de la teoría del caos y la complejidad. Dicha teoría se sustenta sobre 4 pilares básicos:

1. Principio de la incertidumbre: la imprevisibilidad de un sistema complejo, cuyo comportamiento no puede no puede ser previsto a largo plazo.

2. Principio de globalidad: En contraposición con el reduccionismo clásico.

3. Principio de la interdependencia: el funcionamiento de cada elemento depende del de los demás y cualquier modificación afecta al conjunto.

4. Principio de la emergencia espontánea: de las interacciones múltiples entre elementos aislados emerge una nueva entidad global (un sistema complejo), que es diferente que la simple suma de sus elementos. Cuándo hablamos de ciencias de la complejidad nos referimos a los campos que estudian científicamente los 
sistemas complejos y el comportamiento complejo. Podemos decir que las ciencias de la complejidad (Balague, Torrents, Hristovski, Davids, \& Araújo, 2013) buscan formas de organización en los sistemas, sinergias buscan leyes o principios de comportamientos generales enfatizan el estudio de las interacciones y la conectividad del sistema (entre sus diferentes componentes y con otros sistemas)

Han aparecido publicaciones actuales (Krustrup \& Krustrup, 2018) donde se ha planteado el deporte como recurso dentro de un modelo holístico, en el cual los patrones de movimiento y las respuestas fisiológicas, generan adaptaciones relacionadas con la aptitud física y la salud inducidas por el entrenamiento.

Desde otro punto de vista, quizá mas tangible, fisiológico y funcional, Gabbet \& Whiteley (2017) expusieron el modelo "Paradoja de Prevención de Lesiones de Entrenamiento" (figura 17) con el cual muestran que los deportistas acostumbrados a cargas de entrenamiento altas tienen menos lesiones que los deportistas que entrenan con cargas de trabajo más bajas.

Este modelo basado en la evidencia, ha indicado que las lesiones sin contacto no son causadas por el entrenamiento sino más bien por un programa de entrenamiento inapropiado. Los aumentos excesivos y rápidos de las cargas de entrenamiento son probablemente responsables de una gran proporción de lesiones de tejidos blandos sin contacto. Si la carga de entrenamiento es un determinante importante de la lesión, debe medirse con precisión hasta dos veces al día y durante toda la temporada. 


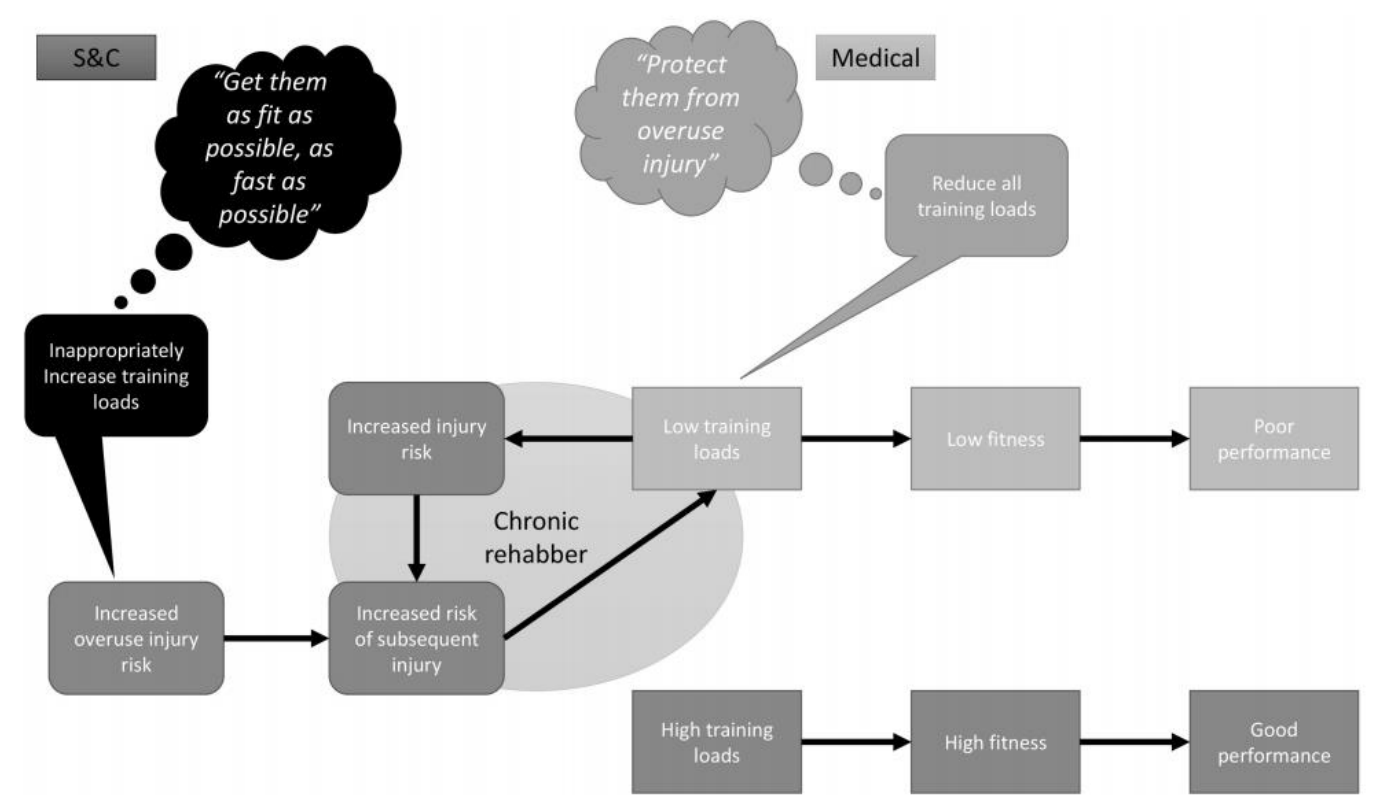

Figura 17. Modelo de prevención de lesiones basado en el entrenamiento con cargas óptimas (Gabbett \& Whiteley, 2017)

En el aspecto de la prevención, para finalizar, hacer referencia a la necesidad de conocer las demandas que suponen para los deportistas los entrenamientos y las competiciones. Ello nos indica en qué medida el entrenamiento reproduce lo que sucede durante la competición. A pesar de que ningún trabajo ha evaluado con exactitud y especificidad si las demandas físicas del partido son replicadas adecuadamente en el entrenamiento en futbolistas varones (Carling et al., 2008) esto sí que se ha realizado en otros deportes colectivos como el fútbol australiano (Dawson, Hopkinson, Appleby, Stewart, \& Roberts, 2004), el rugby (Hartwig, Naughton, \& Searl, 2011) o el hockey (Gabbett, 2010) obteniendo diferencias, como cabía esperar, entre entrenamientos y partidos. Así, por ejemplo, en variables tales como: el tiempo invertido en zonas de media y alta velocidad (Gabbett \& Mulvey, 2008; Wehbe, Hartwig, \& Duncan, 2014a), la distancia recorrida (Henderson, Cook, Kidgell, \& Gastin, 2015) o la frecuencia de sprints 
(Dawson et al., 2004), se han obtenido mayores valores durante los partidos respecto a los entrenamientos.

\subsubsection{Programas de rehabilitación / readaptación en lesiones isquiosurales}

Las tasas persistentemente altas de nuevas lesiones observadas en las lesiones de isquiosurales han sugerido que los programas de rehabilitación llevados a cabo hasta la fecha podrían ser insuficientes (Ekstrand et al., 2016).

Tras la amplia revisión realizada en el apartado 1.2 sobre las lesiones de isquiosurales, hemos observado que los programas de rehabilitación utilizados han estado alejados de contenidos específicos del deporte, especialmente en las fases de readaptación y reentrenamiento deportivo. De hecho, algunos autores han indicado la necesidad de que las propuestas y programas incluyan tareas de reeducación del movimiento y aspectos condicionales (reentrenamiento) en contextos específicos del futbolista (Bosch, 2016; Castellano \& Casamichana, 2013; Taberner \& Cohen, 2018).

Es decir, todavía en la actualidad existe la necesidad de programas que incluyan pruebas funcionales, y reentrenamiento multiplanar que evalúen los parámetros de rendimiento en relación con la recuperación después de las lesiones de isquiosurales, especialmente en los momentos de readaptación y reentrenamiento a nivel deportivo específico.

Nuestro estudio, ha elaborado un modelo de readaptación de acuerdo a esta necesidad de incluir una mayor especificidad y adecuación a las necesidades concretas del fútbol y se ha validado como modelo de readaptación en lesiones de isquiosurales (Jiménez-Rubio, Navandar, Rivilla-García, \& Paredes-Hernández, 2018).

Respecto a la búsqueda de herramientas para la prevención y readaptación de lesiones del deporte encontramos autores que han publicado en esta línea (Bosch, 2016; Lalín \& 
Peirau, 2011) y dieron luz a la terminología sobre Reeducación Funcional Deportiva (RFD) (Lalín Novoa, 2008a, 2008b).

La RFD es la descripción de un proceso que abarca desde el inicio del accidente deportivo hasta la vuelta completa a la práctica. A ello se le deben añadir los programas preventivos para evitar recaídas e incluso para anticiparse a la aparición del accidente deportivo.

Tal y como se observa en la figura 18, el proceso que integra los acontecimientos del entorno de la lesión no es lineal sino circular, ya que, la aparición de la lesión, es cierto que desencadena un proceso donde se hará necesaria la planificación de un trabajo de readaptación, para la vuelta a la práctica deportiva, pero a la vez, un programa de prevención deberá ser integrado para cerrar el círculo, y mantener al deportista en la competición el mayor tiempo posible.

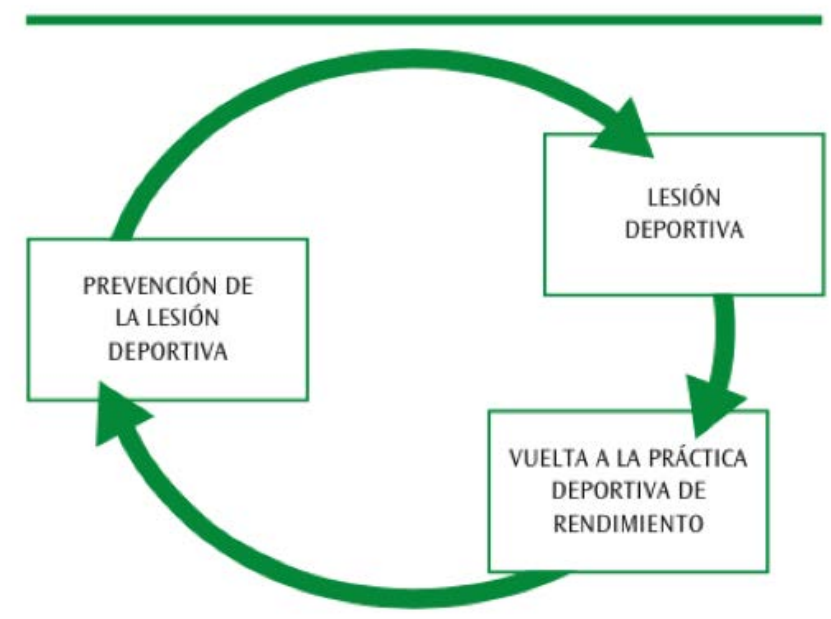

Figura 18. Modelo explicativo proceso circular prevención, abordaje lesión y prevención terciaria (post-lesión) (Lalín \& Peirau, 2011)

Es importante reseñar que la finalidad última del proceso de rehabilitación, readaptación y vuelta a la práctica es reeducar los movimientos y gestos deportivos que el jugador ya 
realizaba con maestría anteriormente y que se han visto entorpecidos por la aparición de la lesión, habilidades que debe volver a ejecutar con el mismo nivel de eficacia anterior. Ello requiere generar a su vez al tejido lesionado nueva capacidad de tolerancia al estímulo/esfuerzo que será fundamental conocer y evaluar en el proceso.

El proceso de rehabilitación debe comenzar con un diagnóstico preciso por parte del profesional médico, quien también propondrá la estrategia terapéutica a seguir (ya sea conservadora o quirúrgica). Una vez que se ha diseñado y puesto en marcha la propuesta de tratamiento, se deberán respetar unos procesos biológicos de cicatrización tisular, que van a requerir de una temporalidad. En esta línea, deberán favorecerse los procesos reparadores del organismo, reduciendo así las posibilidades de recaída (Järvinen et al., 2007; Järvinen \& Kalimo, 2013).

En esta primera etapa el objetivo será devolver a las estructuras afectadas su funcionalidad perdida (Bayer, Magnusson, Kjaer, \& Tendon Research Group Bispebjerg, 2017). Para ello, las diferentes propuestas de fisioterapia ayudarán en gran medida a recuperar una posible pérdida del balance articular y el balance muscular, mitigar los dolores o procesos inflamatorios mediante técnicas de electroterapia, etc. Superada esta primera etapa (recuperación) de objetivos eminentemente clínicos, dará comienzo la segunda etapa, donde los objetivos son de tipo más funcional y hacen referencia a la condición física y a la especialidad deportiva (Lalín \& Peirau, 2011; Lalín Novoa, 2008a, 2008b; Mendiguchia et al., 2017).

Se puede establacer que, una vez finalizada la etapa de recuperación, el deportista es capaz de desarrollar actividades de la vida diaria con relativa facilidad (andar, subir y bajar escaleras, etc.) que van a permitir dar al deportista un alta médica.

No obstante, este sujeto practicante todavía presenta déficits evidentes para retornar a la 
práctica deportiva, y más aún para enfrentarse a la exigencia de una práctica deportiva de rendimiento. Por ello, el objetivo de esta segunda y tercera etapa (la readaptación y reentrenamiento, el objeto de esta intervención en la tesis) es alcanzar un alta deportiva que capacite al jugador a volverse a entrenar en las mismas condiciones que el resto de compañeros no lesionados.

Por otra parte, se encuentran varios trabajos que justifican la necesariedad del entrenamiento de fuerza en los programas de readaptación en lesiones del deporte (FortVanmeerhaeghe et al., 2016; Martínez-valencia, Romero-arenas, \& Elvira, 2015; Reiman \& Lorenz, 2011; Romero-Rodriguez, Gual, \& Tesch, 2011).

Además, en relación a la readaptación deportiva, recientemente (2016) en la comunidad de Madrid, se ha aprobado la ley 6/2016, de 24 de noviembre, de ordenación de profesiones del deporte, donde queda tipificado que el Grado en ciencias de la actividad física y del deporte es el perfil académico legal para desarrollar planes de readaptación físico-deportiva.

Teniendo en cuenta las escasas investigaciones donde se han diseñado y validado programas de rehabilitación y readaptación para la lesión a nivel proximal de los isquiosurales, en la presente tesis doctoral, se ha realizado un diseño y una validación de un modelo de entrenamiento para la lesión concreta miotendinosa proximal isquiosural. Igualmente, valorando la escasez de estudios que hayan analizado la eficacia de un programa de readaptación, esta tesis doctoral ha estudiado y analizado los efectos de los parámetros físicos post-lesión del deportista lesionado. 
Para ello, según vemos en la figura 19, se tuvo en cuenta el concepto actual de reentrenamiento del deportista de élite, que debería estar basado en esta imagen.

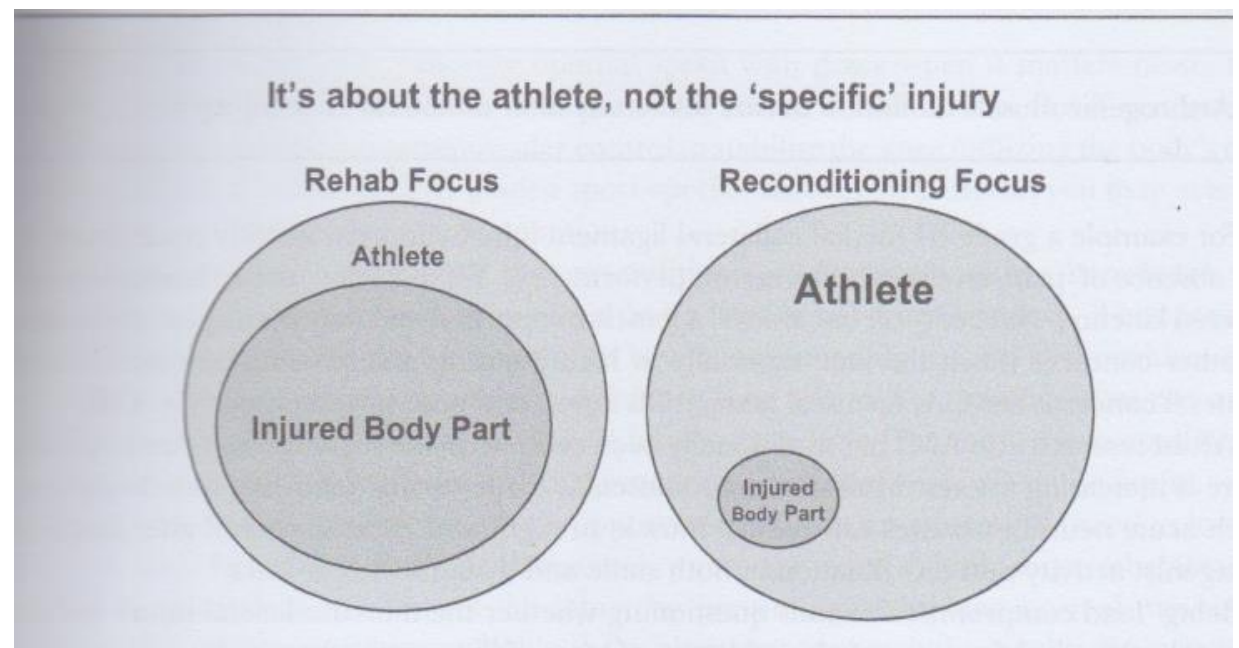

Figura 19. El Acondicionamiento del deportista es más amplio que la propia lesión (Joyce \& Lewinton, 2016)

\subsection{La herramienta de medición del estudio: el GPS}

En esta tesis doctoral se utilizó el sistema "Global Positioning System" (GPS) para realizar las mediciones, concretamente con el modelo SPI Elite, (GPSports Systems, Fishwick, Australia), de 5 hz y 80 gramos de peso.

En relación al GPS, la literatura muestra que autores como Campos (2015) y De Hoyo et al. (2016) publicaron tesis doctorales y trabajos de investigación utilizando diferentes modelos de GPS como elemento tecnológico para medir y cuantificar variables de rendimiento en equipos de fútbol profesional.

Mediciones realizadas en relación a parámetros de aceleración/desaceleración en función del tiempo de entrenamiento y en función de variable repetición de sprints “repeated sprint ability” (RSA), variables características del juego del fútbol.

Otros trabajos igualmente también registraron variables con GPS en fútbol de alto rendimiento como distancias a diferentes velocidades y carga metabólica (Lacome, 
Simpson, Cholley, Lambert, \& Buchheit, 2018; Padulo et al., 2017; Røksund et al., 2017; Taylor, Macpherson, Spears, \& Weston, 2015).

En la actualidad existen sistemas incluso más sofisticados como los "local positioning system" (LPS). Los cuales no proporcionan una cobertura global y son utilizados por diversas casas comerciales para ampliar el alcance de los GPS y proporcionar información donde las señales de éstos no pueden llegar.

\subsection{1 ¿Por qué monitorizar la carga de entrenamiento?}

El control de la carga tiene como objetivo proporcionar constante información acerca del sistema que se controla. Una evaluación precisa de la carga de entrenamiento, que es un parámetro importante para la planificación y periodización del entrenamiento.

En los deportes colectivos como el fútbol, la carga de entrenamiento prescrita por el entrenador es a menudo denominada carga externa (CE) a través del volumen de carga soportado en el microciclo entre otros, mientras que se denomina carga interna (CI) al estrés fisiológico soportado por el jugador, a través de frecuencia cardíaca, encuestas de RPE ó VO2 max (Jaspers, Brink, Probst, Frencken, \& Helsen, 2017; Tibana et al., 2018).

Sin embargo, en este tipo de deportes explosivos, nos encontramos con una dificultad añadida para valorar la carga de entrenamiento, aunque afortunadamente en la actualidad los sistemas de captura y análisis de la información está bastante bien organizado con deportistas de élite.

Dado el escaso tiempo que los entrenadores disponen para preparar los partidos de competición, cada vez es más habitual la utilización de tareas de entrenamiento que integran contenidos físicos, técnicos y tácticos. Este tipo de contenidos, habitualmente 
dan lugar respuestas físico- fisiológicas muy variables, debido a las diferentes funciones (roles tácticos) desempeñadas por los jugadores. Esta elevada variabilidad podría reducir la probabilidad de que los jugadores reciban entrenamientos basados en sus características individuales (Alexiou \& Coutts, 2008) pudiendo dar lugar a respuestas o efectos de entrenamiento no deseados, especialmente, en cuanto a deficits de fuerza que sirvan de profilaxis.

Para intentar evitar esos efectos de entrenamiento no deseados (que pueden ser diferentes para cada sujeto), se necesita de un adecuado control de la carga de entrenamiento, mediante la monitorización de las respuestas físicas y fisiológicas individuales. La capacidad de medir y monitorizar las cargas de entrenamiento debe proporcionar información valiosa para la prescripción de programas de entrenamiento individualizados (Borresen \& Ian Lambert, 2009) y para prevenir procesos de sobreentrenamiento (Halson, 2014).

Concretamente, el estudio de las relaciones individuales entre diferentes variables relacionadas con la carga de entrenamiento (CI, CE, fatiga, etc....), podría ayudar a establacer la dosis de entrenamiento adecuada para cada jugador, con el objetivo de optimizar el rendimiento (Manzi, Bovenzi, Franco Impellizzeri, Carminati, \& Castagna, 2013) y reducir la incidencia de lesión (Gabbett \& Jenkins, 2011).

Impellizzeri, Rampinini, y Marcora (2005) muestran unas interesantes orientaciones sobre las relaciones entre las variables mencionadas. Según los autores, el resultado del entrenamiento depende de la CI soportada por el deportista (estrés fisiológico). Esta CI está determinada por la CE (estímulos físicos que realiza el deportista) y por las características individuales del jugador (edad, condición física, composición corporal...). Sin embargo, la dosis de CI no será la única variable determinante del resultado de 
entrenamiento.

\subsubsection{Medios de control de la carga de entrenamiento}

En la actualidad existen multitud de posibilidades para controlar la carga soportada durante las sesiones de entrenamiento en los deportes de equipo. Diversos métodos basados en el análisis de la frecuencia cardíaca como el método de Edwards ,TRIMP de Stagno (Stagno, Thatcher, \& van Someren, 2007) o TRIMP individualizados (Manzi, Iellamo, Impellizzeri, D’ottavio, \& Castagna, 2009) se utilizan de forma habitual como forma de control de la CI en estudios realizados con futbolistas (Scott, Lockie, Knight, Clark, \& Janse de Jonge, 2013). Además, el uso de dispositivos GPS dan información sobre numerosas variables relacionadas con la $\mathrm{CE}$, como distancia total, distancia relativa, patrones de movimiento en diferentes zonas de velocidad, aceleraciones y desaceleraciones... (Cummins, Orr, O’Connor, \& West, 2013).

Sin embargo, todos estos dispositivos, en ocasiones, presentan importantes limitaciones como su alto coste económico o el elevado tiempo necesario para realizar los complejos análisis, si se busca una exhaustiva pormenorización de variables.

En los últimos años, se ha generalizado el uso de la percepción subjetiva del esfuerzo ("Rate of perceived exertion”, RPE) para el control de la carga de entrenamiento en los deportes de equipo (Impellizzeri, Rampinini, Coutts, Sassi, \& Marcora, 2004; Tibana et al., 2018) Se trata de una herramienta barata, práctica y precisa para valorar las respuestas individuales a las sesiones de entrenamiento de campo .

El método Session RPE propuesto por Foster (Foster et al., 2001) se basa en el uso de una escala de Borg-10 mediante la que los jugadores determinan la intensidad del entrenamiento 30 minutos después de finalizarlo. Nos encontramos por tanto ante un 
método de control que por su fácil aplicación y escaso coste puede ser utilizado con jugadores de diferentes edades o nivel deportivo.

\subsubsection{Utilización de tecnología GPS para el control del riesgo de lesión}

Hasta hace pocos años, el principal objetivo del uso del GPS ha sido identificar los perfiles de actividad de los jugadores en competiciones o entrenamientos. Sin embargo, en los últimos tiempos ha habido un creciente interés del uso del GPS (Al Haddad, Méndez-Villanueva, Torreño, Munguia-Izquierdo, \& Suárez-Arrones, 2017; Buchheit, Al Haddad, et al., 2014; Varley, Fairweather, \& Aughey, 2012) para mantener el equilibrio entre el estrés neuromuscular provocado por la carga de entrenamiento y la recuperación del mismo, maximizando así una potencial mejora del rendimiento y minimizando el riesgo de sobreeentrenamiento examinado.

Al mismo tiempo, este uso del GPS también se ha realizado con objeto de intentar relacionar el vínculo entre las variables asociadas a los GPS y las lesiones del tejido blando sin contacto que se producen en jugadores profesionales de fútbol.

\subsubsection{Utilización de tecnología GPS para el control en readaptación}

Viendo la literatura existente (De Hoyo \& Aceña Rodríguez, 2017) una de las principales decisiones que se debe tomar durante el proceso de readaptación de un deportista lesionado es usar dispositivo GPS y con ello determinar el momento que está preparado para la competición. Es complejo para el equipo técnico, encontrar el equilibrio correcto entre incorporar a un jugador demasiado pronto con el consiguiente riesgo de recurrencia, o retardar su vuelta, con las consiguientes repercusiones deportivas que ello conlleva.

En este sentido, un aspecto crítico que debe tenerse presente a la hora de tomar la decisión de que el jugador retorne, se relaciona con la cantidad y calidad de 
entrenamiento que dicho jugador ha completado durante el tiempo de la recuperación para estar adecuadamente preparado para las demandas propias del juego.

Bajo esta perspectiva, la propuesta del control de carga en el deportista lesionado de Blanch y Gabbet (2016) nos puede servir de referencia para tomar esta decisión. De esta forma, una proporción de carga de trabajo A:C de 0,5 sugeriría que un deportista entrenó o completó solo la mitad de la carga de trabajo en la semana más reciente en comparación con lo que había preparado para las 4 últimas semanas.

Una proporción de carga de 2,0 sugiere que el deportista realizó el doble de carga de trabajo en la semana actual que lo que había preparado en las 4 últimas semanas. En dicho trabajo (Blanch \& Gabbet, 2016) se propoinen como principales variables a tener presente en el análisis, la distancia total recorrida y la distancia a alta intesidad, la superior a $18 \mathrm{~km} / \mathrm{h}$. Muestran como "picos" periodos en los que el jugador tiene una carga de trabajo A:C excesiva. En ambos casos, se ha establecido como nivel óptimo para reducir la presencia de recidivas de lesiones el ratio de 0,8 a 1,4 (Blanch \& Gabbett, 2016).

Por otro lado, otro parámetro que puede ayudarnos durante el proceso de readaptación del futbolista lesionado es la asimetría en el apoyo (p.ej parámetro step balance). Así el hecho de controlar la presencia de asimetrías (a través del acelerómetro) es esencial cuándo se está realizando un proceso de readaptación post-lesión (Buchheit, Gray, \& Morin, 2015). Un ratio A:C por encima de la zona de riesgo, conllevará un aumento de la fatiga (central y periférica) asociada con una mayor alteración de las variables del paso de carrera y las fuerzas verticales (De Hoyo et al., 2016), manifestándose variaciones caóticas en asimetrías de carrera. En esta línea Buccheit et al. (2015) comprobaron como un incremento del ratio A:C conllevaba un aumento en la asimetría por encima de la zona de estabilidad (+- 2,5). 
Desde hace décadas, la monitorización de los movimientos desarrollados por los deportistas durante el entrenamiento o la competición esta siendo un tema de interés para los científicos del deporte (Carling et al., 2008; Castellano \& Casamichana, 2014) El seguimiento realizado posibilita conocer los requerimientos físicos a los que son sometidos los jugadores (Barbero-Alvarez, Coutts, Granda, \& Castagna, 2010; De Hoyo et al., 2016) permitiendo intervenir de una forma específica en el entrenamiento y evaluar el rendimiento durante las competiciones (Barros et al., 2007; Buchheit, Allen, et al., 2014; Di Salvo et al., 2010).

En este sentido, diversas tecnologías se han utilizado para el registro del movimiento de los deportistas (Casamichana \& Castellano, 2011). Laboriosas técnicas de registro manual (Reilly \& Thomas, 1976), han ido evolucionando hacia grabaciones magnetofónicas, tabletas digitales o softwares específicos (Bloomfield et al., 2007) llegando a los sistemas más modernos como las técnicas de registro semiautomático a través de video o videotracking. Entre estos últimos, los más conocidos, los sistemas AMISCO francés (Castellano \& Álvarez, 2013), ProZone inglés (Di Salvo, Gregson, Atkinson, Tordoff, \& Drust, 2009) y los menos, como el TRACAB sueco o el Verusco de Nueva Zelanda.

Actualmente existen alternativas que permiten el registro de manera totalmente automática, es decir, sin intervención humana como el Venetrack que es un sistema de videotracking (Redwood-Brown, Cranton, \& Sunderland, 2012), los sistemas basados en radiofrecuencia (Frencken, Lemmink, \& Delleman, 2010), los sistemas LPS, los tracking ó los sistemas de posicionamiento global (Global Positioning System o GPS), siendo, como hemos visto, éstos últimos unos de los más extendidos en la actualidad con una gran producción científica (Aughey, 2011; De Hoyo et al., 2016). 
Habitualmente, los dispositivos receptores portátiles GPS se introducen en una pequeña mochila almohadillada (arnés o chaleco) incorporada a la espalda del jugador, justo debajo del cuello (explicado en apartados 3.2.2 y 3.4). Además, estos dispositivos permiten registrar datos referentes al tiempo, posición, altitud y dirección, además de registrar la frecuencia cardiaca cuándo el jugador está en posesión de una banda torácica.

En consecuencia, el espectro de información registrado por los dispositivos es muy amplio y son convertidas en multitud de variables de diferente índole, tales como: distancia recorrida (en metros y expresada en términos relativos a la distancia total recorrida) o duración de carrera (en segundos o en términos relativos al total), distancia o tiempo de desplazamiento a diferentes rangos de velocidad de carrera, velocidad instantánea, media y máxima, work/rest ratio, frecuencia de carreras a diferentes intensidades, saltos, acciones de alta intensidad repetidas, carga metabólica o compensacón de apoyos (Buchheit, Méndez-Villanueva, Delhomel, Brughelli, \& Ahmaidi, 2010) por poner algunos ejemplos.

Todas ellas, variables que tienen que ver con el espacio, el tiempo, y sus derivaciones. Hace algunos años con la implementación del acelerómetro en los dispositivos GPS se ha abierto una nueva dimensión en la monitorización y cuantificación de la carga , a partir, sobre todo, de indicadores globales como el "player load" (Castellano, Casamichana, Calleja-González, Román, \& Ostojic, 2011) o el "total body load” (Gómez-Piriz, Jiménez-Reyes, \& Ruiz-Ruiz, 2011), parámetros diseñados por las marcas comercializadoras del producto.

Conocedores de las variables que puede llegar a aportar los GPS, el uso primario y más común de estos dispositivos en el mundo del deporte (especialmente en deportes de situación) ha sido describir los desplazamientos y perfil de esfuerzos que realizan los 
deportistas durante la competición, extendiéndose el uso de esta tecnología a multitud de deportes como el fútbol (Barbero-Alvarez et al., 2010, 2015; Buchheit, Haddad, et al., 2014), fútbol sala (Castagna \& Barbero Álvarez, 2010), fútbol playa (Casamichana \& Castellano, 2010), fútbol-7 (Castellano, Casamichana, et al., 2011), fútbol australiano (Dawson et al., 2004; Ruddy et al., 2016), rugby (Gabbett \& Jenkins, 2011; Gabbett, Jenkins, \& Abernethy, 2012; Reid, Cowman, Green, \& Coughlan, 2013), hockey (Gabbett, 2010; White \& MacFarlane, 2013), cricket (Petersen, Pyne, Portus, \& Dawson, 2009) y tenis (Galé-Ansodi, Castellano, \& Usabiaga, 2013).

Hasta el momento de la autorización de uso en competición en el año 2015, existía la prohibición del uso de los dispositivos GPS en partidos oficiales de competición en algunos deportes (como es el caso del fútbol). Ello provocó que su aplicación se extendiese, sobre todo, en el proceso de entrenamiento (Hill-Haas, Dawson, Impellizzeri, \& Coutts, 2011).

La monitorización de los jugadores en el entrenamiento permite conocer en qué medida el entrenamiento replica las demandas impuestas a los jugadores durante la competición (Casamichana \& Castellano, 2011), observándose en general que durante los entrenamientos no se reproducen las demandas de alta intensidad de los partidos (Lago, Casais, Domínguez, \& Sampaio, 2010; Suárez-Arrones et al., 2015a).

De ahí que esos y otros trabajos se hayan centrado en la evaluación de las demandas físicas de los jugadores mediante la utilización de la tecnología GPS durante diferentes situaciones de juegos reducidos (SSG), a los que se les ha modificado las dimensiones del terreno de juego, número de jugadores, duraciones, orientación del espacio, número de toques permitidos por jugador, entre otros (Dellal, Drust, \& Lago-Peñas, 2012; HillHaas et al., 2011; Lacome et al., 2018). 


\subsubsection{Aplicación del GPS en el Fútbol de alto rendimiento}

Los primeros intentos que se hicieron para validar GPS en aplicaciones para el ámbito deportivo datan de 2006 y se basaron en la validación de la precisión del GPS como instrumento para medir el movimiento en diferentes rangos de velocidad. Mientras que el GPS ha sido validado para aplicarlo en deportes de equipo, siguen existiendo algunas dudas sobre la conveniencia del GPS para medir movimientos cortos de alta velocidad. No obstante, el GPS se ha aplicado ampliamente en el fútbol australiano, cricket, hockey, rugby y fútbol (Aughey, 2011; Varley et al., 2012; Varley, Gabbett, \& Aughey, 2014) Hay una extensa información sobre el perfil de actividad de los deportistas de deportes de equipo en la literatura derivada de GPS, que aporta información sobre distancia total recorrida por jugadores y distancia diferenciada en rangos de velocidad. El GPS también se ha aplicado para detectar fatiga en partidos, identificar los períodos de juego más intensos, perfiles de actividad diferente por la posición y el nivel de competición del deporte (Al Haddad et al., 2017; Aughey, 2011; Varley et al., 2014).

La validación adicional de GPS para el deporte de equipo no ocurrió hasta 2009-2010, con una serie de estudios que emplean metodología y tecnologías GPS (Aughey, 2011; Coutts \& Duffield, 2010; Portas, Harley, Barnes, \& Rush, 2010).

Una comparación directa a través de estos estudios es difícil, si el objetivo es una declaración para respaldar la validez del GPS en los deportes de equipo, debido a la variedad dispositivos GPS, de tareas y ejercicios analizados, velocidades de muestreo y métodos estadísticos aplicados (Aughey, 2011).

Varias investigaciones (Duffield, Reid, Baker, \& Spratford, 2010) coinciden en afirmar que cuanto mayor sea la velocidad de muestreo del GPS más válido será para medir la distancia recorrida. Si comparamos tareas similares de alta velocidad realizadas en 
distintos estudios, comprobamos como el error en la distancia estimada es mayor en dispositivo GPS de $1 \mathrm{~Hz}$ que en uno de $5 \mathrm{~Hz}$ (Aughey, 2011) y en este es mayor que el obtenido en un GPS de 10 Hz (Aughey, 2011; Castellano \& Casamichana, 2010).

Es evidente que ante velocidades de muestreos reducidas, la velocidad del movimiento a registrar influye directamente en la validez de la medida. Sin embargo, cuanto más larga sea la duración de una tarea que se mide con el GPS, se convierte en más valida la medición de esa distancia (Aguiar, Melbourne, \& Scrivner, 2009) Realizando una actividad de alta intensidad en fútbol de $197 \mathrm{~m}$ simulado, se apreció error de un $1.5 \%$. De hecho, en distancias más largas, incluso el GPS de $1 \mathrm{~Hz}$ ofrecía resultados con una validez elevada sobre la distancia recorrida (Barbero-Alvarez et al., 2010; Jennings, Cormack, Coutts, Boyd, \& Aughey, 2010b).

La aplicación de la actual tecnología GPS es posible en las medidas de mayor duración, incluso si contienen períodos de actividad de alta velocidad utilizados en para evaluar la capacidad máxima de sprint en jóvenes futbolistas (Buchheit et al., 2010).

Además, investigadores y profesionales deben tender a utilizar GPS con una mayor tasa de muestreo para mejorar la validez de los resultados.

La fiabilidad del GPS para determinar análisis cinemático de los deportistas está influenciada por diferentes factores: la velocidad de muestreo, la duración de la tarea y el tipo de tarea, (Jennings, Cormack, Coutts, Boyd, \& Aughey, 2010a; Portas et al., 2010). El efecto que se produce por una mayor velocidad de muestreo, hace que el GPS ofrezca resultados ambiguos en cuanto a fiabilidad (Aughey, 2011).

Un estudio nos muestra que el coeficiente de variación $(\mathrm{CV})$ de un sprint de $10 \mathrm{~m}$, fué del $77 \%$ con $1 \mathrm{~Hz}$ GPS y $39.5 \%$ con $5 \mathrm{~Hz}$, sin embargo, en las tareas de fútbol lineal, el 
CV fué 4.4 - 4.5\% de $1 \mathrm{~Hz}$ y 4.6 5.3\% para $5 \mathrm{~Hz}$ (Aughey, 2011; Portas et al., 2010).

Menos ambigüedad encontramos en la afirmación, que cuanto mayor sea la velocidad de movimiento, menor será la fiabilidad del GPS. El CV aumentó de 30.8\% a 77.2\% para un sprint de $10 \mathrm{~m}$ en comparación con caminar sobre la misma distancia medida con GPS de $1 \mathrm{~Hz}$. Usando un GPS de $5 \mathrm{~Hz}$, el CV aumentó $23.3 \%$ a 39.5\% para la misma tarea a las mismas velocidades (Jennings et al., 2010b; Varley et al., 2012).

Varios estudios han establecido una buena validez para el GPS de $1 \mathrm{~Hz}$ en el establecido de la distancia media obtenida durante una actividad de movimiento simulado de deportes de equipo (Aughey, 2011; Portas et al., 2010).

Sin embargo, la fiabilidad de los ensayos lineales y específicos de fútbol en los dispositivos de $1 \mathrm{~Hz}$ y $5 \mathrm{~Hz}$ estuvieron dentro del 5\% de coeficiente de variación, que le hacen cumplir con los criterios establecidos como fiables en estudios similares (Ruddy et al., 2016) En conclusión, la fiabilidad de los dispositivos GPS depende de la tarea y el tiempo que debe considerarse al informar sobre las diferencias individuales de los deportistas del equipo (Aughey, 2011; Varley et al., 2012).

Hay otro aspecto, en ocasiones olvidado en comparación con validez y fiabilidad y es el número y colocación de satélites para la recepción de los datos.

En la actualidad, los dispositivos GPS, son pequeños y ligeros, añadido esto, su capacidad de almacenamiento (hasta 4 horas), permite una buena adaptación a los deportes de equipo. Hasta la fecha ninguna investigación anterior ha evaluado la aplicación de tecnología GPS a patrones de locomoción específicos que caracterizan el rendimiento de la élite del fútbol (Portas et al., 2010).

Según Osgnach, et al. (2010), la tecnología GPS podría utilizarse en el fútbol profesional 
para abrir nuevos caminos de cara al entrenamiento para una mejor enumeración de las tareas y dar especificidad a la carga de entrenamiento, así como también para el análisis de partidos jugados. De hecho, la tecnología GPS tiene el potencial para proporcionar un examen más completo, preciso y automatizado de los movimientos del jugador en deportes de equipo (Jennings et al., 2010a, 2010b).

Tal es así que la tecnología del GPS se utiliza actualmente en deportes del equipo para cuantificar demandas del movimiento en el entrenamiento y la competición. Esta información se puede entonces utilizar para modificar el tipo, la duración y la intensidad del entrenamiento, mejorando la especificidad de estas sesiones (Jennings et al., 2010a, 2010b).

Los sistemas de "Time Motions Analisys", tales como el GPS no miden las exigencias de la competición, sino los desplazamientos de los jugadores. El análisis de las cargas externas proporciona información valiosa sobre las necesidades fisiológicas y de rendimiento de deportistas de deportes de equipo.

Entrenadores y científicos del deporte utilizan medidas de distancia de partidos o ejercicios de entrenamiento para evaluar las demandas de trabajo y comparar el rendimiento de los deportistas de deportes de equipo (Jennings et al., 2010b). Estos análisis han demostrado siempre que la distancia recorrida a alta intensidad depende de la posición de juego, el nivel de la competencia, la capacidad física del jugador, y el rendimiento físico del oponente (Gaudino, Alberti, \& Iaia, 2014; Rampinini et al., 2009).

Por ejemplo, hay claras diferencias en el rendimiento de los futbolistas de élite cuándo el marcador de partido representa a un equipo empatando, perdiendo o ganando (Aughey, 2011). Lo que no se puede asegurar es el cumplimiento de las exigencias físicas de los jugadores, así como su estado de fatiga en el juego (Duffield et al., 2010). Sin embargo, 
no está claro si los mismos patrones de fatiga pueden ser detectados en partidos con diferentes sistemas de análisis (Randers et al., 2010).

Para poder sacar más aplicación al GPS, la extensión lógica es la de dividir el movimiento en rangos de velocidad, o incluso intentar localizar los periodos, donde se produzca acciones de alta intensidad en los partidos, así como la repetición de esas secuencias de intensidad (Buchheit, Haddad, et al., 2014).

La continua aplicación de la tecnología GPS para la recopilación de datos generados en deportes de equipo puede permitir un desafío para el reconocimiento de que la capacidad repetida de sprint es crucial para los deportistas de deportes de equipo en un futuro cercano (Ruddy et al., 2016).

Con la utilización de la tecnología GPS, se llega a obtener un mejor conocimiento de las características del tipo de movimiento que desarrollan los deportistas tanto en la competición como en el entrenamiento.

Una mayor comprensión de los partidos y entrenamientos nos va a permitir la confección de tareas adaptadas a los jugadores y a los perfiles de actividad requeridos en los partidos (Aughey, 2011; Ruddy et al., 2016).

Entre otros aspectos, el uso del GPS, es claramente un importante elemento que cuantifica la carga externa del deportista (Bourdon \& Cardinale, 2017).

Dicho uso justifica estrategias preventivas para evitar lesiones de isquiosurales (Duhig et al., 2016) ó por ejemplo para valorar y controlar volúmenes de entrenamiento a velocidades de alta intensidad $(>24 \mathrm{~km} / \mathrm{h})$. 


\subsubsection{Fiabilidad/ Validez de los GPS}

Diferentes estudios han analizado la validez y fiabilidad de receptores GPS durante la locomoción humana en un ámbito recreativo (Schutz \& Herren, 2000; Terrier, Turner, \& Schutz, 2005; Townshend, Worringham, \& Stewart, 2008).

También lo han aplicado en contextos más específicos de los deportes colectivos (Castellano, Casamichana, et al., 2011; Castellano, Fernández, Castillo, \& Casamichana, 2010; Coutts \& Duffield, 2010; Edgecomb \& Norton, 2006) donde se estimaron diferentes resultados debido a la utilización de diversos dispositivos GPS, frecuencias de muestreo, algoritmos utilizados con la valoración de diferentes variables (velocidad media, máxima, posiciones, desplazamientos) o diferentes protocolos a realizar como trayectorias rectas, curvilíneas, cambios de dirección a diferentes velocidades, etc. (MacLeod, Morris, Nevill, \& Sunderland, 2009), pero en general obteniéndose en la mayoría de los casos resultados satisfactorios con su utilización y manejo.

Para realizar la comparación entre diferentes trabajos debemos tener presente el modelo de dispositivo que se utiliza en estudios donde evalúan aceleraciones y desaceleraciones (Castellano \& Casamichana, 2013; Coutts \& Duffield, 2010; Petersen et al., 2009) debido a los algoritmos asociados a cada uno de ellos (MacLeod et al., 2009).

La mayoría de estudios desarrollados en el ámbito del rendimiento deportivo en deportes de equipo han utilizado dispositivos que operan con una frecuencia de muestreo de $1 \mathrm{~Hz}$ (Barbero-Alvarez et al., 2010; Coutts \& Duffield, 2010; Gray, Jenkins, Andrews, Taaffe, \& Glover, 2010), otros han comparado unidades de GPS de 1 y 5 Hz (Castellano, Casamichana, et al., 2011; Castellano et al., 2010; Jennings et al., 2010b) y otros han analizado únicamente dispositivos GPS de $5 \mathrm{~Hz}$ (Casamichana \& Castellano, 2011; Johnston, Gibson, et al., 2013) ó de 10 Hz (Castellano, Casamichana, et al., 2011; De 
Hoyo et al., 2016) y otros han sido los trabajos que han comparado dispositivos de $5 \mathrm{y}$ 10 Hz (Johnston, Watsford, Pine, Spurrs, \& Sporri, 2013; Varley et al., 2012).

A groso modo, parece que una mayor frecuencia de muestreo podría aumentar la precisión de los datos aportados por estos aparatos (Castellano \& Casamichana, 2013; Duffield et al., 2010).

En estos estudios citados, los GPS analizados por Castellano et al. (2011) o De Hoyo et al. (2016), que tuvieron una frecuencia de muestreo de $10 \mathrm{~Hz}$ obtuvieron mejores resultados en carreras de $30 \mathrm{~m}$ que los obtenidos en trabajos anteriores (Duffield et al., 2010; Petersen et al., 2009), cuándo analizaron los dispositivos con una frecuencia de muestreo de 1 y $5 \mathrm{~Hz}$.

Otros autores (Johnston, Watsford, et al., 2013) también valoraron la fiabilidad, precisión y validez de los nuevos dispositivos que operan con frecuencias de muestro de $5 \mathrm{~Hz}$ y compararon con los ya existentes de $10 \mathrm{~Hz}$, sin encontrarse mejoras reseñables o conclusiones significativas.

En estudios recientes (Hoppe, Baumgart, Polglaze, \& Freiwald, 2018) se muestra incluso, errores en dispositivos en función de la frecuencia utilizada, siendo estos de $5 \mathrm{hz}$ con fiabilidad para registrar, y puntualizando dispositivos de hasta $20 \mathrm{hz}$ con mayores posibilidades de validez.

La fiabilidad y validez del GPS spi Pro x (Gpsports), fué demostrada en estudios previos con jugadores de otros deportes, cricket (Petersen et al., 2009) y de igual forma, estudios muy actuales continúan utilizando estos mismos dispositivos (Lacome et al., 2018; Stares et al., 2018).

El registro de los parámetros de rendimiento de las competiciones registradas así como las tareas durante la fase de intervención de las lesiones de la unión miotendinosa proximal del bíceps femoral, se llevó a cabo mediante este sistema de posicionamiento 
global - "Global positioning system” (GPS) usando el modelo SPI Elite, (GPSports Systems, Fishwick, Australia), de 5 hz y 80 gramos de peso.

El sistema GPS esta sincronizado a través de señales de radio con 24 satélites en órbita alrededor de la tierra, cada uno de ellos es capaz de emitir con alta precisión la posición y el tiempo exacto. Las señales que reciben dos satélites son convertidas en distancias. De esta manera el software, Team AMS 2.0 (GPSports Systems, Fishwick, Australia) puede fácilmente calcular la distancia hasta cada satélite y conocer la velocidad a la que viaja la señal (figura 22) y se desarrolla en imágenes de la sesión de entrenamiento (figura 24).

\subsection{Propósito del estudio}

El estudio se realizó principalmente con una triple intención.

Por una parte se intentó diseñar y validar un modelo de readaptación y reentrenamiento que pudiese ser útil y reproducible para otros profesionales que se deban enfrentar a la lesión en unión miotendinosa proximal del bíceps femoral con deportistas que realicen tareas y gestos explosivos, con futbolistas profesionales que han sufrido dicha lesión, incluso que pueda ser utilizado, con las correspondientes adaptaciones de tareas y situaciones, a lesiones de otra índole.

Dicha validación se constató a través de un panel de 15 expertos (Tabla 7).

La segunda intención del estudio, fué la de establecer criterios claros de superación de las diferentes etapas de recuperación durante la lesión, desde que el deportista se lesiona hasta que se integra al entrenamiento normalizado con equipo.

Dichos criterios se establecieron a través del propio programa (tablas 5-6).

Como tercera intención, fué propósito del estudio dejar registrado lo que ocurre en los procesos de readaptación de lesiones de este tipo con deportistas de alto nivel. 
Este análisis ha supuesto acercar dos vertientes (ámbito académico y ámbito entrenamiento "alto rendimiento") con la dificultad que conlleva poder hacer mediciones y propuestas a nivel investigación con deportistas que están en constante necesidad de mejorar aspectos del juego y en constante competición.

Dichos registros son los resultados de la presente tesis doctoral (apartado 4).

En este estudio, la propuesta se realizó en una lesión muscular concreta (unión miotendinosa proximal del bíceps femoral) en donde se llevó a cabo una comparación de trece variables de rendimiento para conocer los posibles cambios y las demandas físicodeportivas entre esfuerzos en diferentes competiciones, pero en variadas situaciones. En estado Pre-lesión (PRE), en la vuelta a la competición o "return to play" (C1) y en competición posterior, pasados entre 6-10 semanas tras esa primera competición (C2). Durante el proceso, también se registró en la intervención (REA) y en la fase de GRUPO. Este desarrollo, está más ampliamente explicado en la metodología (apartado 3.1, diseño del estudio). 



\section{Objetivos e hipótesis}





\subsection{Objetivos Generales}

- Diseñar un programa de entrenamiento en fútbol durante el proceso de readaptación y reentrenamiento de la lesión en la unión miotendinosa proximal del bíceps femoral.

- Analizar la eficacia de dicho programa de entrenamiento en el retorno a la competición y en el plazo de las 6-10 semanas siguientes tras la primera competición post-lesión. 


\subsection{Objetivos Específicos}

- Validar a través de un panel de expertos un programa de entrenamiento funcional en el terreno de juego para la readaptación y reentrenamiento durante una lesión en la unión miotendinosa proximal del bíceps femoral.

- Determinar la utilidad del programa de entrenamiento a través de la implementación del mismo en futbolistas de alto rendimiento.

- Establecer criterios para llevar al deportista desde el momento de la lesión a la fase de entrenamiento con grupo post-lesión y criterios para volver a la competición tras la lesión de la unión miotendinosa proximal del bíceps femoral.

\subsection{Hipótesis}

Teniendo en cuenta que tras revisión bibliográfica no se encontraron estudios previos que sustentasen la posibilidad de verificar la eficacia del modelo de intervención, se estableció la siguiente hipótesis nula:

"No habrá una mejora en los registros relacionados con parámetros físicos en competiciones post-lesión respecto a los valores pre-lesión”" 



\section{Material y método}





\subsection{Diseño del estudio}

Se diseñó y ejecutó un estudio longitudinal prospectivo cuasi-experimental, al no presentar grupo control y hacer mediciones y registros sobre los mismos sujetos (19) en los mismos momentos (5).

El perfil de aleatoriedad de la muestra la marca la aparición de la lesión puesto que únicamente se evaluaron deportistas con una lesión concreta, las de la unión miotendinosa proximal del bíceps femoral (Balius, Pedret, \& Pujol, 2013; Heiderscheit et al., 2010) (ver criterios de inclusión, apartado 3.3.2).

Este concepto de no aletoriedad es lo que define el estudio como cuasi-experimental. Los diseños cuasi-experimentales (Hedrick, Bickman, \& Rog, 1993) tienen el mismo propósito que los estudios experimentales, probar la existencia de una relación causal entre dos o más variables. Cuándo la asignación aleatoria es imposible, los cuasiexperimentos (semejantes a los experimentos) permiten estimar los impactos del tratamiento o programa, dependiendo de si llega a establecer una base de comparación apropiada.

Estudios previos destacaron propuestas de actuación sobre lesiones de isquiosurales e intervención a nivel de reentrenamiento en las mismas (Mendiguchia et al., 2016, 2017; Taberner \& Cohen, 2018; Valle et al., 2015), pero sin evaluar tras intervención. 


\subsection{Variables del estudio}

A continuación se muestran las trece variables dependientes medidas en este estudio (variables del GPS), así como las 2 variables independientes (el momento y el atleta).

\subsubsection{Variables dependientes e independientes}

Tabla 1. Variables dependientes

\begin{tabular}{|l|l|l|}
\hline Variable dependiente & Tipo & Valores \\
\hline V1 & Escalar & $\mathrm{m} / \mathrm{min}$ \\
V2 & Escalar & $\mathrm{m} / \mathrm{min}$ \\
V3 & Escalar & $\mathrm{m} / \mathrm{min}$ \\
№ RSA/min & Escalar & $\mathrm{n}$ - repetición sprints/min \\
№ sprints/min >21km/h & Escalar & $\mathrm{n}$ - sprints/min \\
Acc1 & Escalar & $\mathrm{m} / \mathrm{s} 2$ \\
Acc2 & Escalar & $\mathrm{m} / \mathrm{s} 2$ \\
Avg-speed & Escalar & $\mathrm{Km} / \mathrm{h}$ \\
Max-speed & Escalar & $\mathrm{Km} / \mathrm{h}$ \\
Max-Acc & Escalar & $\mathrm{m} / \mathrm{s} 2$ \\
№ impactos>7.1g & Escalar & $\mathrm{n}$ - impactos \\
Explosive distance & Escalar & $\mathrm{m} / \mathrm{min}$ \\
WTRR & Escalar & Valor absoluto \\
\hline
\end{tabular}

V1: velocidad registrada entre $14.4-19.7 \mathrm{~km} / \mathrm{h} ; \mathbf{V 2}$ :velocidad registrada entre 19.8 y $25.1 \mathrm{~km} / \mathrm{h}$ : V3: velocidad registrada de más de $25.1 \mathrm{~km} / \mathrm{h} ; \mathbf{n}^{\mathbf{0}} \mathbf{R S A} / \mathbf{m i n}$ : número de "repeated sprint ability" por minuto; Acc1: $\mathrm{n}^{\mathrm{o}}$ de aceleraciones y desaceleraciones/min registradas entre $2.9-4 \mathrm{~m} / \mathrm{s} 2 ;$ Acc2: $\mathrm{n}^{\circ}$ de aceleraciones y desaceleraciones/min registradas de más de $4 \mathrm{~m} / \mathrm{s} 2 ;$ Avg-speed: media de velocidad: Max-speed: pico máxima velocidad; "Explosive distance": distancia registrada cuándo aceleración supera $1.2 \mathrm{~m} / \mathrm{s} 2$; WTRR: “work to rest ratio”. $\mathbf{~ m / m i n : ~ m e t r o s ~ / ~ m i n u t o ; ~} \mathbf{m} / \mathbf{s} 2$ : metros por segundo2

Tabla 2. Variables independientes

\begin{tabular}{|l|l|}
\hline Variable independiente & Factor \\
\hline Momento & Fijo \\
\hline Deportista & Aleatorio \\
\hline
\end{tabular}


La relación de variables evaluadas con el dispositivo GPS fueron 13, citadas a continuación

$\checkmark$ metros $/$ min recorridos en la franja de velocidad $14,4-19,7 \mathrm{~km} / \mathrm{h}=\mathrm{V} 1$

$\checkmark$ metros/min recorridos en la franja de velocidad $19,8-25,1 \mathrm{~km} / \mathrm{h}=\mathrm{V} 2$

$\checkmark$ metros $/$ min recorridos al superar $25,1 \mathrm{~km} / \mathrm{h}=\mathrm{V} 3$

$\checkmark \mathrm{n}^{\mathrm{o}} \mathrm{RSA} / \mathrm{min}$ con recuperación incompleta $(<6 \mathrm{seg})$ de ACEL $>3,0 \mathrm{~m} / \mathrm{s} 2$

$\checkmark \quad \mathrm{N}^{\mathrm{o}}$ de sprints $/ \min >21 \mathrm{~km} / \mathrm{h}$

$\checkmark \quad \mathrm{N}^{\mathrm{o}}$ de aceleraciones y desaceleraciones entre 2,9 y $4 \mathrm{~m} / \mathrm{s} 2 / \min$ (Acc1)

$\checkmark \quad \mathrm{N}^{\mathrm{o}}$ de aceleraciones y desaceleraciones superiores a $4 \mathrm{~m} / \mathrm{s} 2 / \mathrm{min}$ (Acc2)

$\checkmark$ Velocidad media $(\mathrm{km} / \mathrm{h}):$ Avg_ speed

$\checkmark$ Velocidad máxima $(\mathrm{km} / \mathrm{h}):$ Max_speed

$\checkmark$ Aceleración máxima (m/s2): Max_Acc

$\checkmark \quad \mathrm{N}^{\mathrm{o}}$ Impactos superiores a $7.1 \mathrm{~g}$

$\checkmark$ "Explosive distance" $(\mathrm{m} / \mathrm{min})$

$\checkmark$ “Work-to-rest ratio" (RD>7.0 km/h / RD $\leq 7.0 \mathrm{~km} / \mathrm{h}$ )

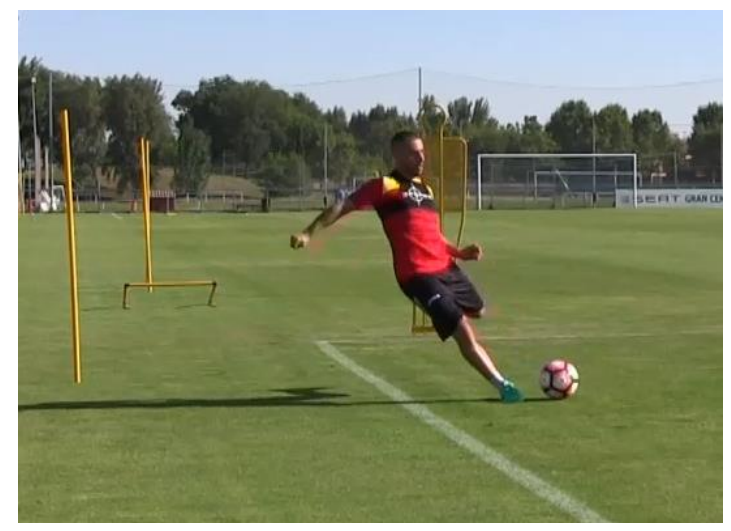

Figura 20. Deportista ejecutando acciones de la intervención con GPS 


\subsubsection{Registro de variables}

Los dispositivos utilizados para el registro de datos en esta tesis doctoral, fueron de la marca Gpsports (Australia) modelo SPI- proX, chip version 2.3 .4 de $5 \mathrm{~Hz}$ con 80 gramos de peso (Buchheit, Al Haddad, et al., 2014; Gaudino et al., 2013; Lacome et al., 2018).

Antes de iniciar el registro correspondiente (entrenamiento o competición), el jugador se coloca el dispositivo, situado en la parte postero-superior de la espalda, a la altura de las 2 primeras vértebras dorsales. El gps se inserta en un chaleco de tela preparado para tal efecto y que rodea los hombros del jugador para evitar movimientos durante el ejercicio físico y asegurar el registro de los impactos (figura 21).

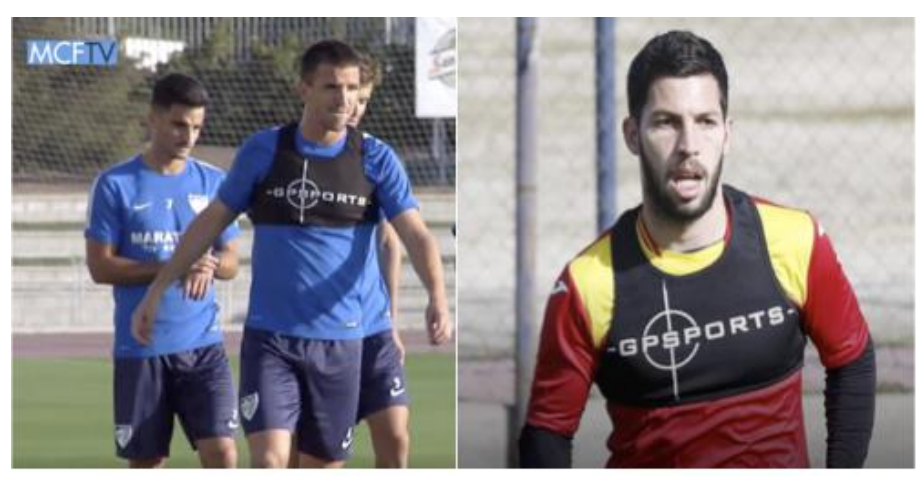

Figura 21. Chaleco y dispositivo (GPS) para registrar parámetros físicos del juego

Una vez finalizadas los momentos de registro de datos, estos se descargaron y se trataron con ese mencionado software Team AMS v.2.0 (Buchheit, Allen, et al., 2014; Elgethun, Yost, Fitzpatrick, Nyerges, \& Fenske, 2006; Petersen et al., 2009). 


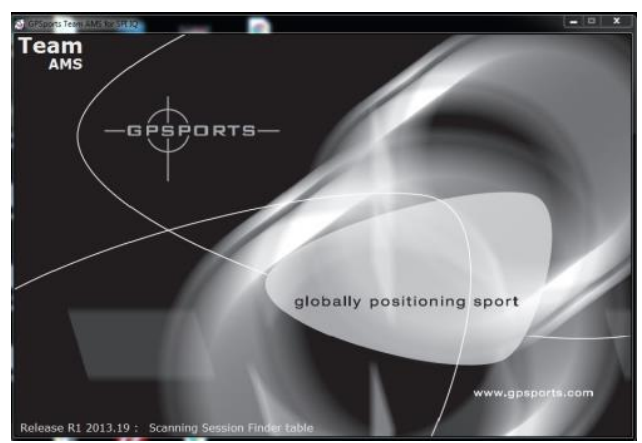

Figura 22. Software Team AMS v.2.0

Con ello se determinaron cuantitativamente las 13 variables dependientes (Tabla 1), para el análisis del rendimiento.

El software utilizado (figura 22) fué TEAM AMS V. R1 2013.19.

Los registros de cada una de las variables se obtuvieron como se describe a continuación en figura 23:

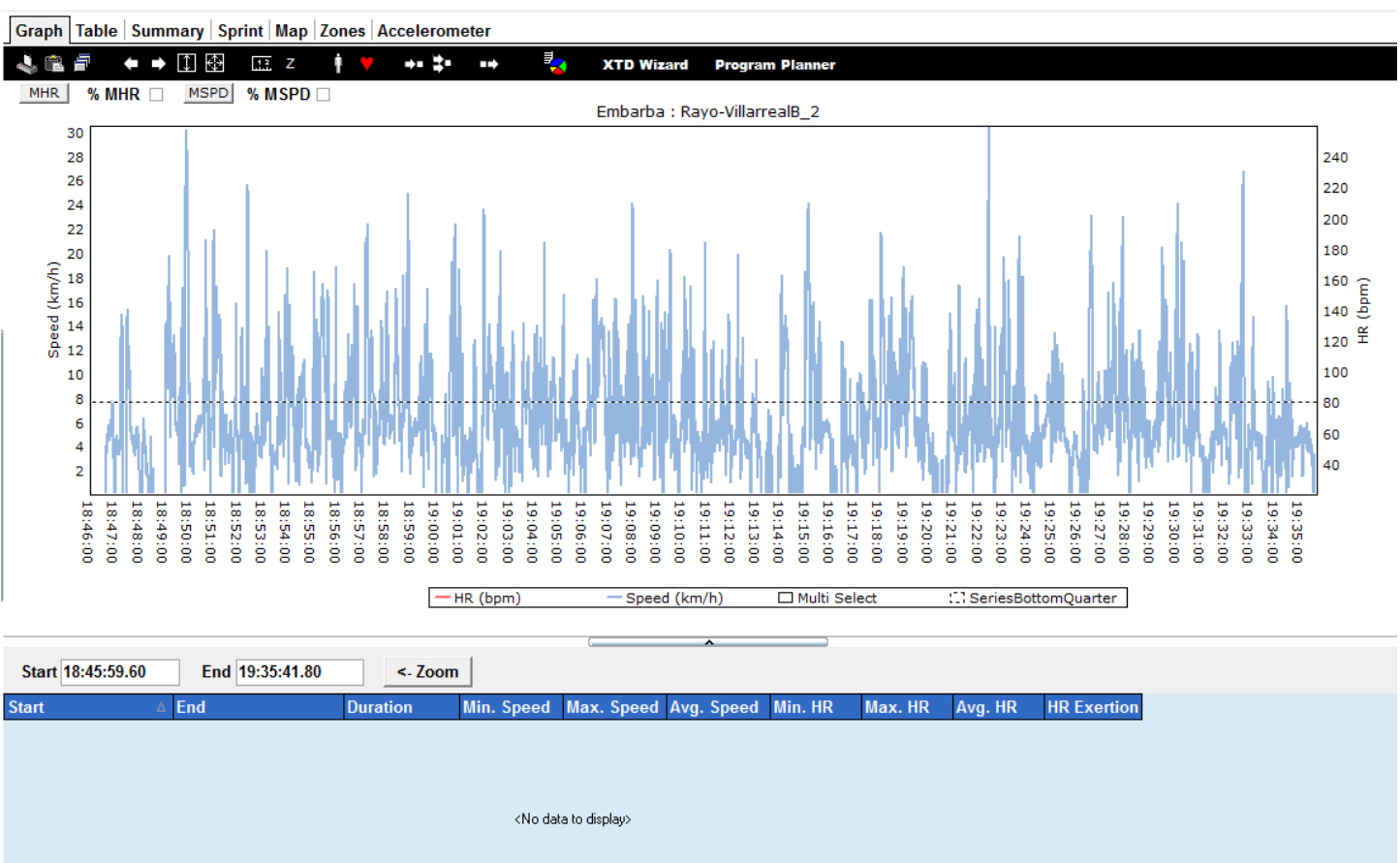

Figura 23. Imagen de registro de sesión/competición con dispositivo GPS 
3.2.2.1 Variables $\mathrm{m} / \mathrm{min}$ a diferentes velocidades (V1,V2,V3)

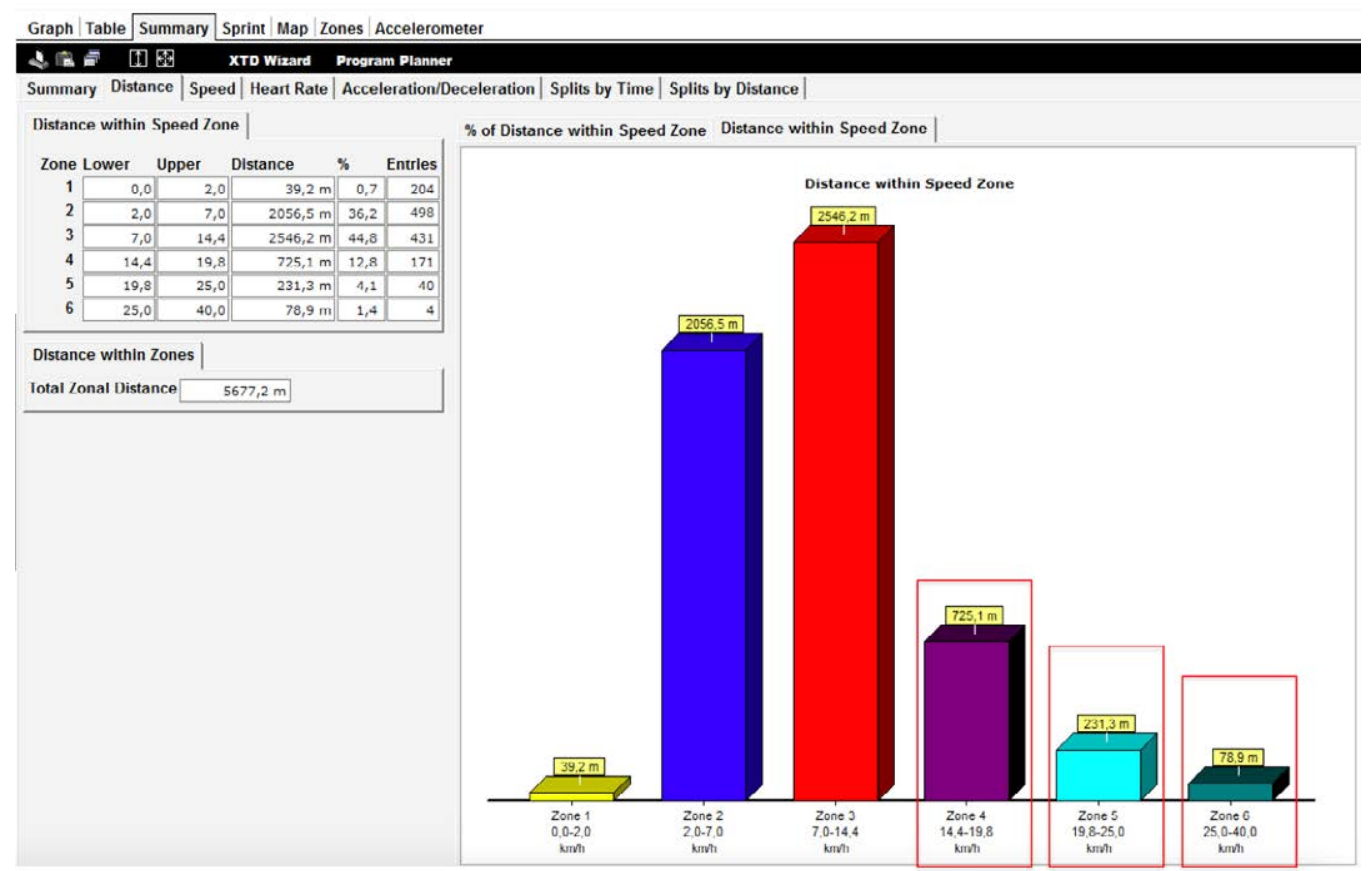

Figura 24. Obtención de m/min a V1 (14.4-19.7) ; V2 (19.8-25.1)

y V3 $(>25.1 \mathrm{~km} / \mathrm{h})$

Evaluando $\mathrm{m} / \mathrm{min}$ en 3 velocidades diferentes (figura 24) según describe la literatura en "high intensity", "very high intensity" y en "sprint" (Di Salvo et al., 2009) y donde diferentes estudios han usado estos mismos parámetros en cuanto a velocidades $\mathrm{m} / \mathrm{min}$ a $14.4-19.7 \mathrm{~km} / \mathrm{h}=\mathrm{V} 1 / 19.8-25.1 \mathrm{~km} / \mathrm{h}=\mathrm{V} 2 /$ más de $25.1 \mathrm{~km} / \mathrm{h}=\mathrm{V} 3$ para registrar a sus deportistas (Bradley \& Noakes, 2013; Di Salvo et al., 2007, 2009; Gaudino et al., 2013; Gleason, Sams, Salley, Pustina, \& Stone, 2017; Lacome et al., 2018; Mallo, Mena, Nevado, \& Paredes, 2015; Stevens, De Ruiter, Twisk, Savelsbergh, \& Beek, 2017; Wehbe et al., 2014a).

El criterio definitivo para elegir estas variables de velocidad, pasó por registrar por encima de $\operatorname{los} 24 \mathrm{~km} / \mathrm{h}$, que es la velocidad aceptada en sistemas tracking actuales (p.ej Mediacoach ${ }^{\circledR}$ ) como la velocidad sprint, y dado que se encontraron trabajos que lo registraron, se decidió utilizar estos rangos. 
3.2.2.2 Variables Vel maxima (Max_speed), Vel media (Avg_speed)

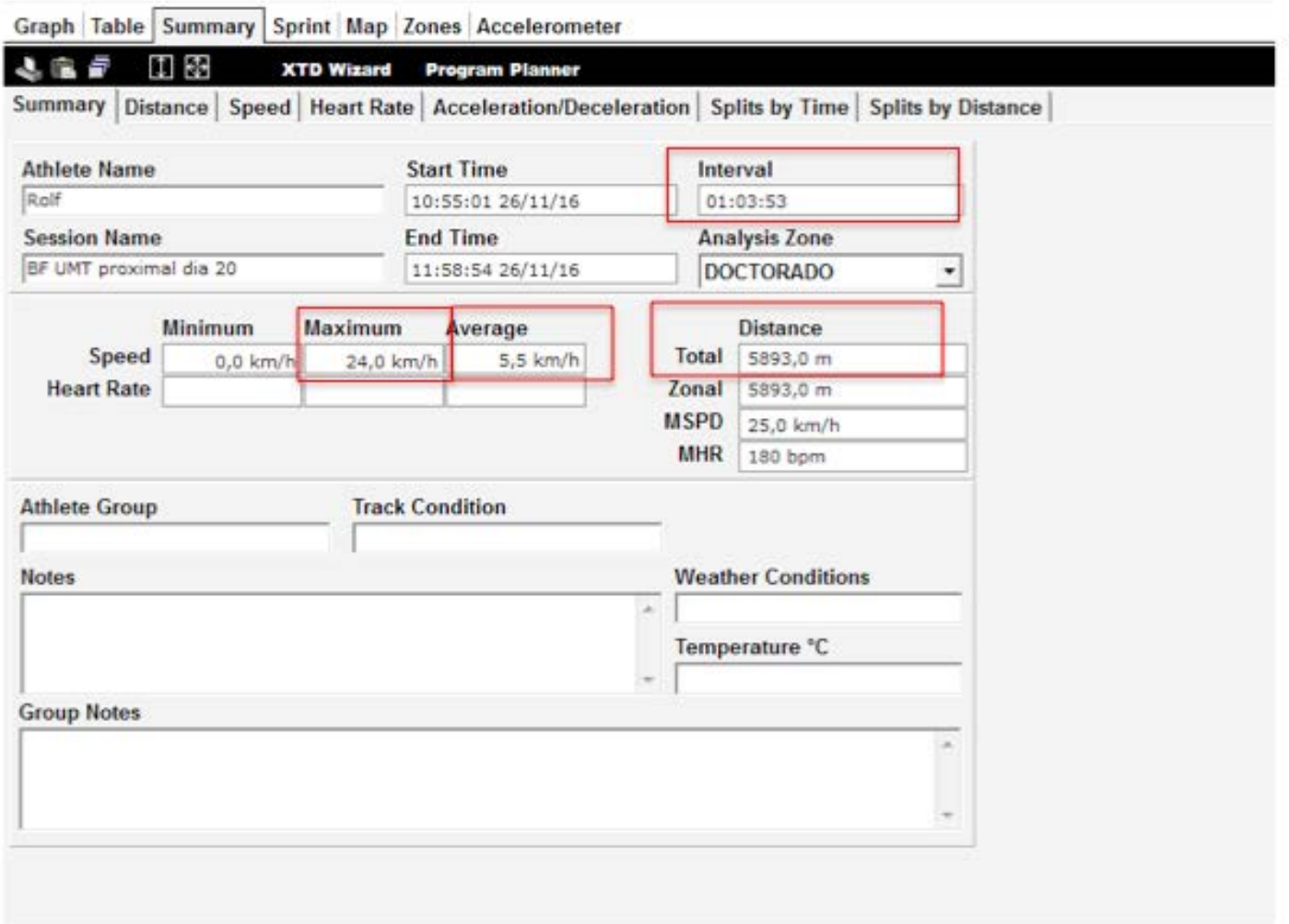

Figura 25. Obtención de distancia recorrida, velocidad máxima y velocidad media (Avg_speed)

Otros autores usaron parámetros como la velocidad máxima (figura 25), así la literatura muestra (Little \& Williams, 2005) trabajos donde se incluyeron a 106 jugadores profesionales de fútbol que fueron evaluados para una carrera sprint de 10 metros (aceleración), una carrera sprint de 20 metros (velocidad máxima) y un rendimiento de agilidad en zigzag. Aunque los rendimientos en las tres pruebas estaban significativamente correlacionados $(p<0,001)$, los coeficientes de determinación $(\mathrm{r}(2))$ entre las pruebas fueron de sólo 39, 12 y 21\% para aceleración y velocidad máxima, aceleración y agilidad, y máxima velocidad y agilidad, respectivamente, vieron que la aceleración, la velocidad máxima y la agilidad eran cualidades específicas y relativamente ajenas entre sí. 
La Avg_speed (figura 25) ha sido medida por otros autores previamente (BarberoAlvarez et al., 2015; Beato, Bartolini, Ghia, \& Zamparo, 2016) al igual que Max_speed (Roecker, Mahler, Heyde, Röll, \& Gollhofer, 2017).

\subsubsection{Variable Work-to-rest ratio $(\mathrm{RD}>7.0 \mathrm{~km} / \mathrm{h} / \mathrm{RD} \leq 7.0 \mathrm{~km} / \mathrm{h})$}

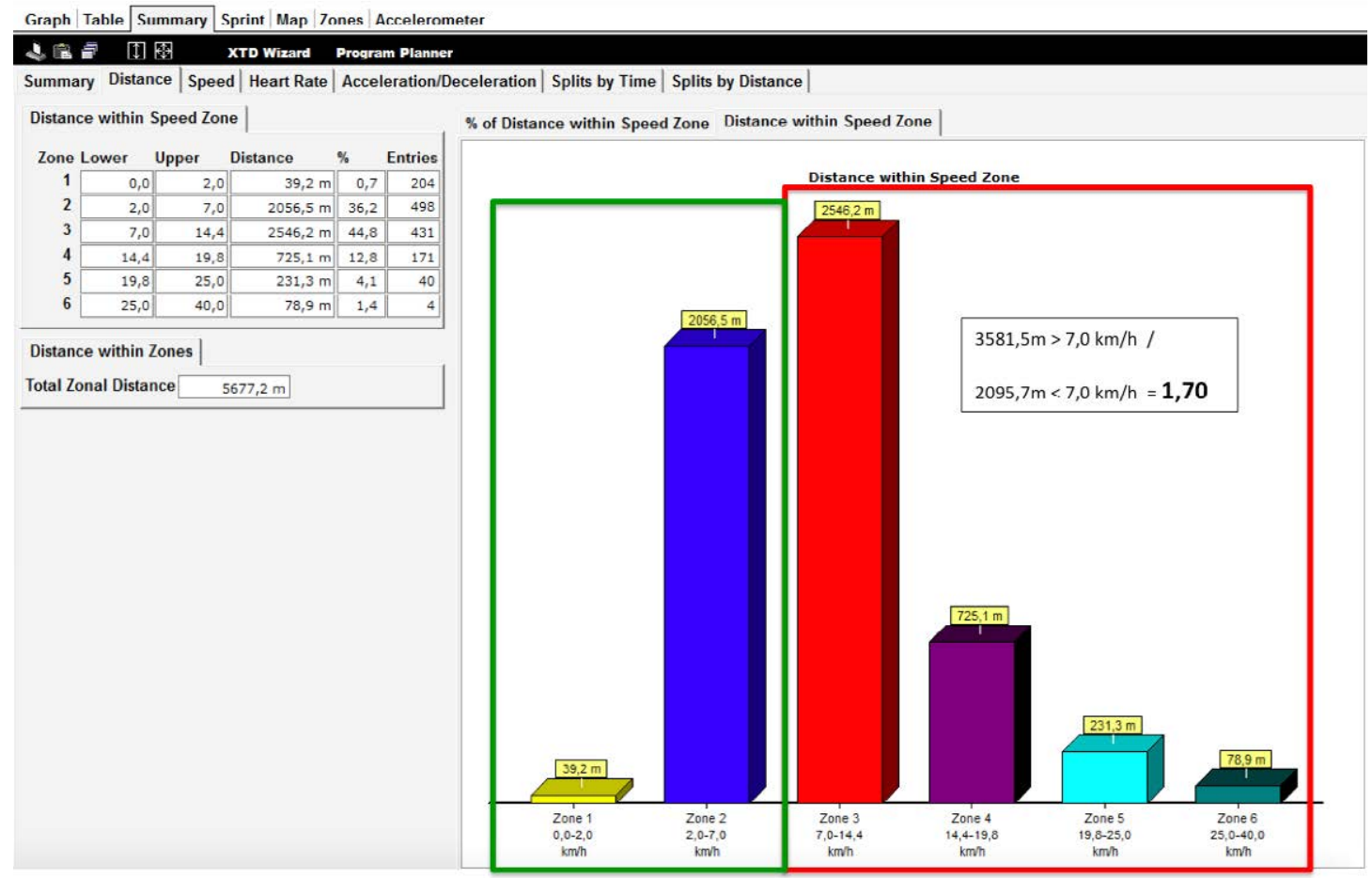

Figura 26. Obtención del parámetro "work to rest ratio" (densidad de esfuerzos)

Es una descripción de la cantidad de trabajo o ejercicio realizado en un intervalo en comparación con la cantidad de descanso. "Work to rest ratio" es una variable que hace referencia a la densidad de los esfuerzos; registra los metros realizados andando, es decir, por debajo de $7 \mathrm{~km} / \mathrm{h}$, respecto a los metros que realiza corriendo, es decir, por encima de 7km/h (Suárez-Arrones, Nuñez, Portillo, \& Méndez-Villanueva, 2012; Suárez-Arrones et al., 2015a) (figura 26) y que en baloncesto también han registrado (Schelling \& Torres, 2016). 
Más estudios que hacen relación entre metros recorridos por encima de $7 \mathrm{~km} / \mathrm{h}$ y metros recorridos por debajo de 7km/h (Tee, Lambert, \& Coopoo, 2016; White \& MacFarlane, 2013).

\subsubsection{Variable $\mathrm{n}^{\mathrm{o}}$ Acel y Desacel $/$ min media intensidad $(2.9-4 \mathrm{~m} / \mathrm{s} 2)$}

Graph $\mid$ Table Summary Sprint $\mid$ Map $\mid$ Zones $\mid$ Accelerometer

\section{Q. 놀}

Summary $\mid$ Distance $\mid$ Speed $\mid$ Heart Rate Acceleration/Deceleration $\mid$ Splits by Time $\mid$ Splits by Distance

Advanced Settings

\begin{tabular}{|c|c|c|c|}
\hline \multicolumn{4}{|c|}{ Acceleration Zone Entries } \\
\hline & Zone 1 & Zone 2 & Zone 3 \\
\hline Acceleration Zones $(\mathrm{m} / \mathrm{s} / \mathrm{s})$ & $0-2,8$ & $2,8-4$ & $4-10$ \\
\hline Acceleration Zone Entries & 517 & 28 & 3 \\
\hline Deceleration Zones $(\mathrm{m} / \mathrm{s} / \mathrm{s})$ & $0-2,8$ & $2,8-4$ & $4-10$ \\
\hline Deceleration Zone Entries & 548 & 41 & 13 \\
\hline
\end{tabular}

Figura 27. Obtención del $\mathbf{n}^{0}$ de Aceleraciones y Desaceleraciones / min de media intensidad $(2.9-4 \mathrm{~m} / \mathrm{s} 2)$

Estudios recientes (Owen, Djaoui, Newton, Malone, \& Mendes, 2017) han estudiado la relación entre número de aceleraciones y desaceleraciones a diferentes intensidades, concretamente en los rangos que se evaluaron en este estudio $2,9-4 \mathrm{~m} / \mathrm{s} 2 \mathrm{y}+\mathrm{de}$ $4 \mathrm{~m} / \mathrm{s} 2$ (figura 27 ).

Igualmente, otros autores (Sonderegger, Tschopp, \& Taube, 2016) también han utilizado estos rangos para evaluar sus aceleraciones y desaceleraciones, también en fútbol profesional, ello justifica la utilización de las variables mostradas en figuras 2728. 
3.2.2.5 Variable $\mathrm{n}^{\mathrm{o}}$ Acel y Desacel $/ \mathrm{min}$ de alta intensidad $(>4 \mathrm{~m} / \mathrm{s} 2)$

Graph $\mid$ Table Summary Sprint $\mid$ Map $\mid$ Zones $\mid$ Accelerometer

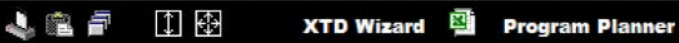

Summary $\mid$ Distance $\mid$ Speed $\mid$ Heart Rate Acceleration/Deceleration $\mid$ Splits by Time $\mid$ Splits by Distance

Advanced Settings

\begin{tabular}{|c|c|c|c|}
\hline \multicolumn{4}{|c|}{ Acceleration Zone Entries } \\
\hline & Zone 1 & Zone 2 & Zone 3 \\
\hline Acceleration Zones $(\mathrm{m} / \mathrm{s} / \mathrm{s})$ & $0-2,8$ & $2,8-4$ & $4-10$ \\
\hline Acceleration Zone Entries & 517 & 28 & 3 \\
\hline Deceleration Zones $(\mathrm{m} / \mathrm{s} / \mathrm{s})$ & $0-2,8$ & $2,8-4$ & $4-10$ \\
\hline Deceleration Zone Entries & 548 & 41 & 13 \\
\hline
\end{tabular}

Figura 28. Obtención del $\mathbf{n}^{0}$ Aceleraciones y Desaceleraciones /min de alta intensidad $(>4 \mathrm{~m} / \mathrm{s} 2)$

En un estudio con ciertas similitudes que el realizado en esta tesis doctoral en número de participantes y en marca y características de los GPS (Wehbe et al., 2014a) se registraron valores de aceleraciones y desaceleraciones $>4 \mathrm{~m} / \mathrm{s} 2$. (figura 28 ).

También en referencia a evaluar lo ocurrido en lesiones del recto anterior, otros estudios cuantifican número de aceleraciones de alta intensidad de esta misma forma (Olmo, Aramberri, Almaraz, Nayler, \& Requena, 2018). 
3.2.2.6 Variable $\mathrm{n}^{\mathrm{o}} \mathrm{RSA} / \mathrm{min}$ con recuperación $(<6 \mathrm{seg})$ y Acel $>3 \mathrm{~m} / \mathrm{s} 2$

no RSA/min con Recuperación incompleta (<6seg) de ACEL>3,0 m/s2

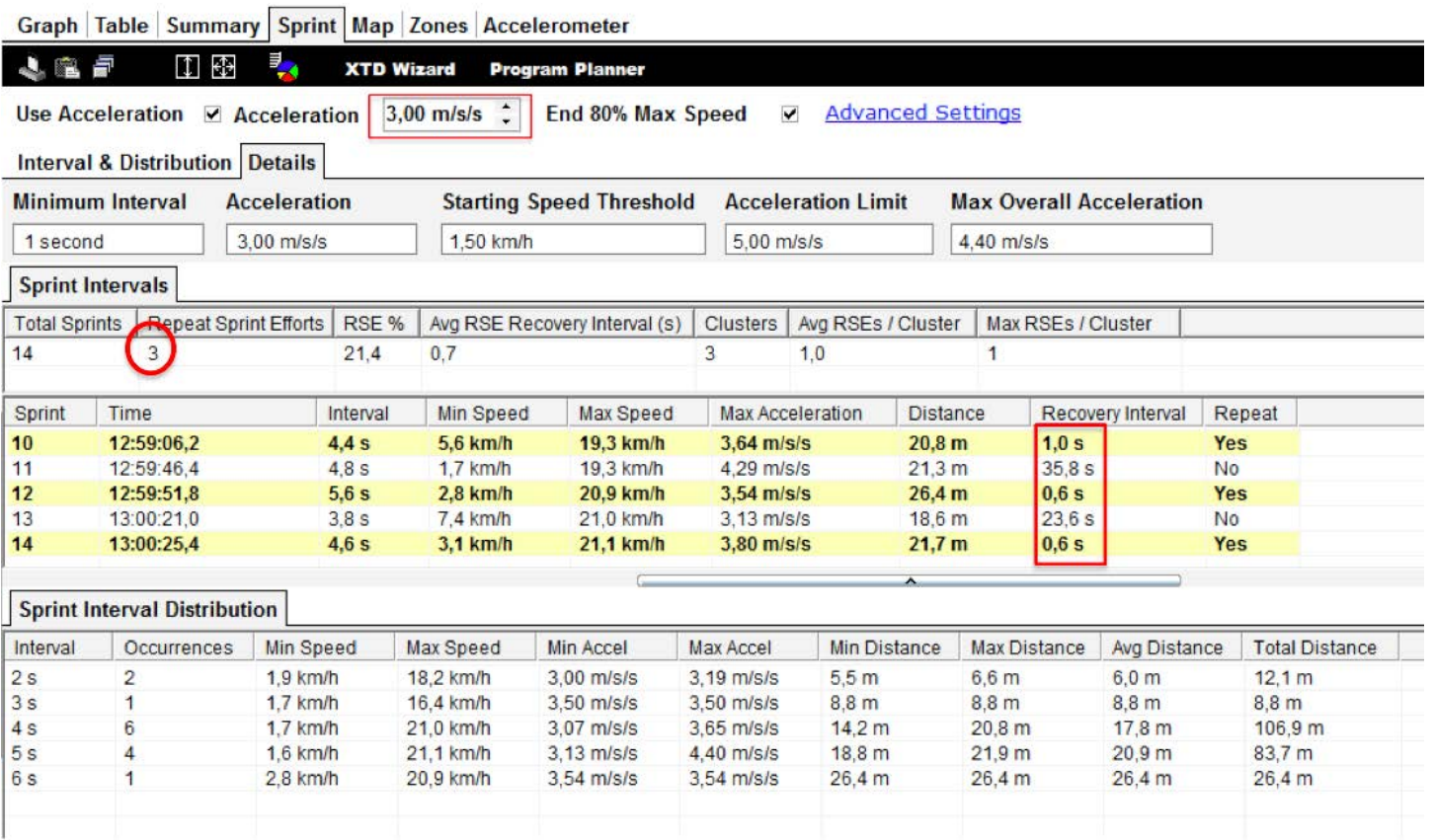

Figura 29. Obtención de número de RSA /min con recuperación ( $<6 \mathrm{seg})$ y aceleración $>$ a $3 \mathrm{~m} / \mathrm{s} 2$

El método RSA, es un método relativamente novedoso. La argumentación científica del método parte del siguiente análisis: en fútbol, se realiza un sprint de 2-4 segundos cada 90 segundos de juego (Stølen, Chamari, Castagna, \& Wisløff, 2005).

Esta densidad de sprints es insuficiente para que el rendimiento se vea comprometido, ya que el tiempo de recuperación es bastante amplio. Sin embargo, los jugadores realizan otro tipo de esfuerzos en estas "recuperaciones" que pueden llevar a la fatiga, tales como contracciones excéntricas, cambios de dirección, carrera a diferentes intensidades.... Además, debido a la naturaleza impredecible del fútbol, períodos cortos donde se sucedan varios sprints pueden ocurrir a lo largo del partido, con una posible incidencia en el resultado del partido, si el organismo no está preparado para 
ello (Spencer, Bishop, Dawson, \& Goodman, 2005), Por ello, el método RSA está basado en la realización de varios sprints de corta duración $(<6$ segundos $)$ con períodos de recuperación muy breves $(<$ de 30 segundos) (Mujika, Spencer, Santisteban, Goiriena, \& Bishop, 2009) el término RSA, difiere de otra propuesta denominada ISE ("Intermittent sprint exercise") (Girard, Méndez-Villanueva, \& Bishop, 2011) que se caracteriza por la realización de sprints de corta duración $(<10$ segundos) con amplios períodos de recuperación (60-300 segundos). La principal diferencia del RSA respecto a ISE, radica en que en éste último hay pequeños o nulos descensos del rendimiento, mientras que en las actividades RSA existe un marcado descenso del rendimiento.

La forma de registrarlo, se muestra en la figura 29.

Otros estudios muy actuales han comparado trabajos de RSA de diferentes duraciones con programas de hasta 5 semanas en deportistas jóvenes con tests como los Yo-Yo intermitent recovery incrementales (Iaia et al., 2017), demostrando con ello que es una variable actual y usada con frecuencia en la investigación de los deportes intermitentes. 
3.2.2.7 Variable $\mathrm{n}^{\mathrm{o}}$ sprints $/ \mathrm{min}>21 \mathrm{~km} / \mathrm{h}$

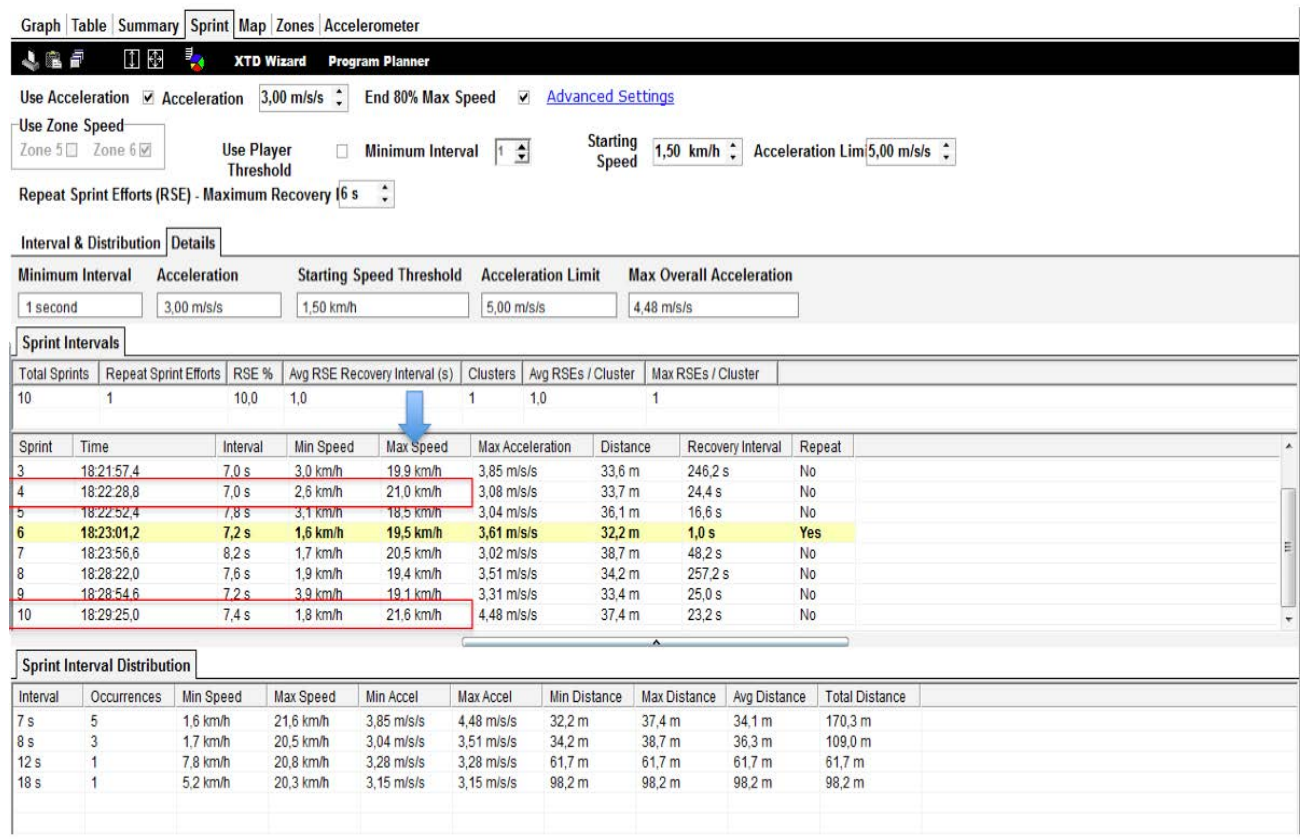

Figura 30. Obtención del número de sprints $/ \mathrm{min}>21 \mathrm{~km} / \mathrm{h}$

Trabajos previos mostraron esta variable (Andrzejewski, Chmura, Pluta, \& Konarski, 2015; Di Salvo et al., 2010), usaremos la variable $n^{\circ}$ de sprints/min cuándo el deportista supera una velocidad de $21 \mathrm{~km} / \mathrm{h}$ y que como se muestra en figura $30 \mathrm{se}$ obtiene de esa forma. En la actualidad, ha sido utilizado el parámetro ${ }^{0}{ }^{\text {sprints }} / \mathrm{min}$ para optimizar el microciclo de entrenamiento desde el punto de vista de evaluarlo y generar parámetros en sesiones desde 5 días previas a la competición (Clemente et al., 2018).

Existen otros estudios que también han medido el número de sprints en competición, en este caso los que superan 24km/h (Miñano-Espín, Casais, Lago-Peñas, \& GómezRuano, 2017). 


\subsubsection{Variable Aceleración máxima}

Graph Table | Summary | Sprint | Map | Zones |Accelerometer

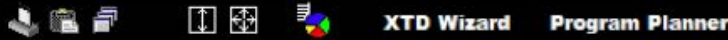

\begin{tabular}{|c|c|c|c|c|}
\hline \multicolumn{2}{|c|}{ Use Acceleration $\vee$ Acceleration } & End $80 \%$ Max Speed & \multicolumn{2}{|c|}{ Advanced Settings } \\
\hline Interval \& Distribution & n Details & & & \\
\hline Minimum Interval & Acceleration & Starting Speed Threshold & Acceleration Limit & Max Overall Acceleration \\
\hline 1 second & $3,00 \mathrm{~m} / \mathrm{s} / \mathrm{s}$ & $1,50 \mathrm{~km} / \mathrm{h}$ & $5,00 \mathrm{~m} / \mathrm{s} / \mathrm{s}$ & $4,76 \mathrm{~m} / \mathrm{s} / \mathrm{s}$ \\
\hline
\end{tabular}

\section{Figura 31. Obtención de la aceleración máxima realizada}

Muchos estudios han usado la variable aceleración máxima (figura 31) para obtener registros con futbolistas profesionales (Akenhead, French, Thompson, \& Hayes, 2014; Bourdon \& Cardinale, 2017; Buchheit, Haddad, et al., 2014) y ese es uno de los motivos por los que se utilizó en esta tesis.

3.2.2.9 Variable número de impactos $>7.1 \mathrm{~g}$

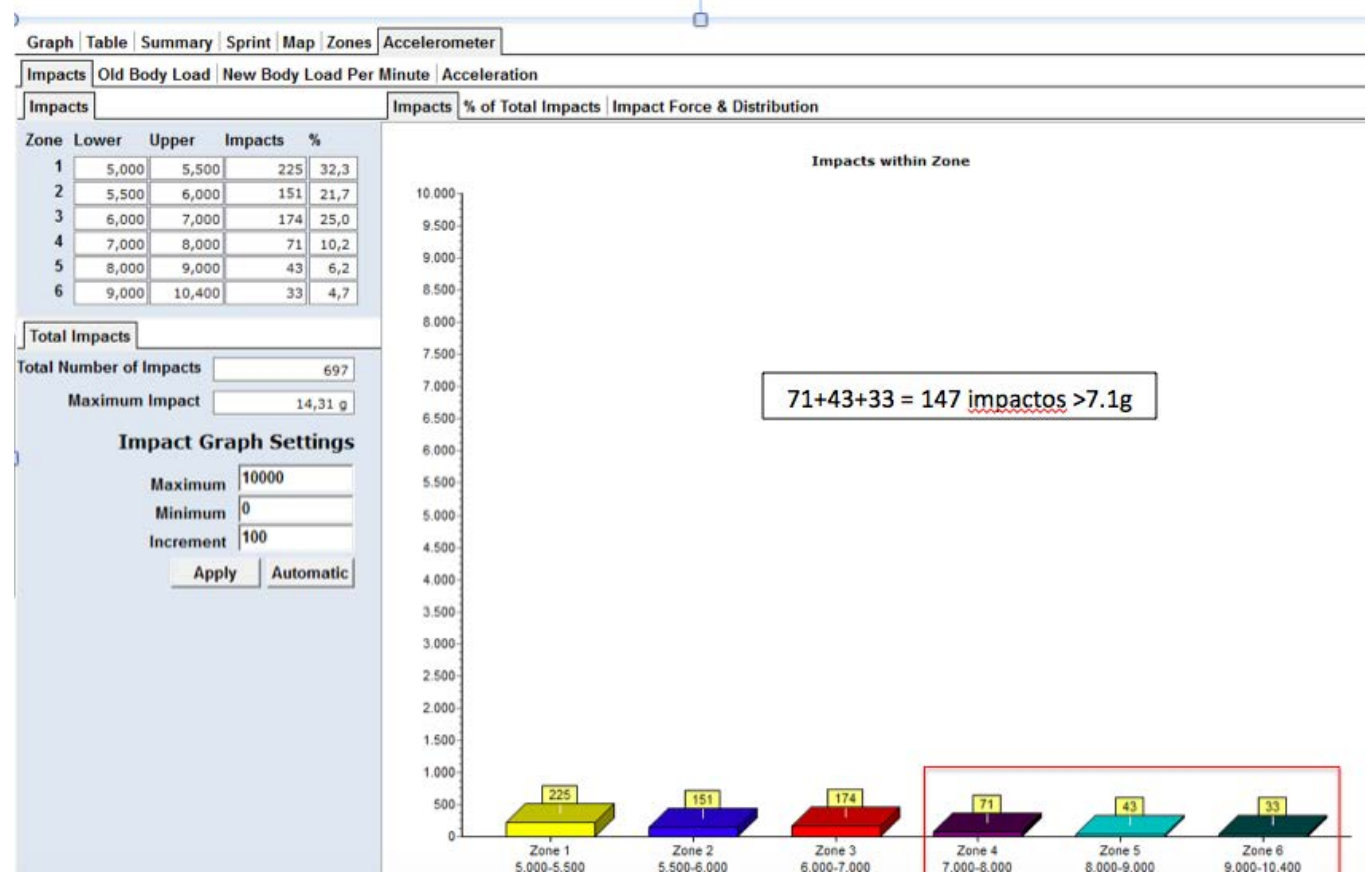

Figura 32. Obtención de número de impactos por encima de 7.1g

Variable medida y analizada similar a como hicieron en otros estudios (De Hoyo et al., 2016). Se muestra su obtención en la figura 32. 
3.2.2.10 Variable Explosive distance ( $\mathrm{m} / \mathrm{min})$

Algunos autores han usado esta variable cuantificando la distancia recorrida por encima de $25.5 \mathrm{~W} / \mathrm{Kg}$ y $\sin$ superar $19.8 \mathrm{Km} / \mathrm{h}$ (Rossi et al., 2016) aunque en esta tesis doctoral, se utilizó el criterio de cuantificar metros/minuto cuándo deportista superaba aceleración de $1.2 \mathrm{~m} / \mathrm{s} 2$ (Ehrmann, Duncan, Sindhusake, Franzsen, \& Greene, 2016).

\subsection{Participantes}

Veintidos (22) futbolistas profesionales, varones y pertenecientes a dos clubes profesionales que militaron en la liga española masculina de fútbol profesional (La Liga) durante 3 temporadas (2015-2018) fueron medidos y registrados, cada uno de ellos a través del sistema "global positioning system" (GPS), en la realización de diferentes competiciones (PRE - C1- C2) y entrenamiento de readaptación (REA) o grupal (GRUPO) durante sus lesiones a nivel proximal en el bíceps femoral, con un perfil de lesión concreto (apartado 3.3.1) descrito en estudios similares previos (Chan et al., 2012; Mueller-Wohlfahrt et al., 2013; Valle et al., 2017).

De estas 22 lesiones en unión miotendinosa proximal del bíceps femoral registradas, 16 de ellas $(72,7 \%)$ se produjeron en competición y 6 de ellas $(31,8 \%)$ se produjeron en entrenamiento, valores muy similares a estudios como el de Duhig et al. (2016). Todos ellos fueron informados por escrito (ver apartado 8.3), al igual que sus clubes de pertenencia firmando a tres partes (investigador-deportista-club de pertenencia) un consentimiento y acuerdo, en el que se explicaba que si en algún momento, de forma voluntaria y sin que supusiese ninguna consecuencia, el deportista podría voluntariamente abandonar la investigación o igualmente estaba a su disposición la elección de no ponerse el dispostivo GPS. 
El estudio, se realizó con la aprobación del Comité de Ética de la UPM- Madrid (ver anexos apartado 8.2 y figuras 50-51).

Según el estudio publicado por Swan et al. (2015) en relación al perfil de la muestra en cuanto a la definición de la experiencia de los participantes "elitelexpert athletes", diremos que en nuestro estudio, se cuenta con una muestra de deportistas de élite calificada como "professional athletes", dado que compitieron en liga profesional masculina en España. (Swann, Moran, \& Piggott, 2015).

En el periodo de tiempo desde Julio de 2015 hasta Junio de 2018, (3 temporadas completas) donde ya no hubo más registros ni intervención cuasi-experimental, de estos 22 futbolistas, 3 de ellos, no cumplieron los criterios de inclusión quedando el estudio por tanto en un $n=19$ definitivo. (figura 33).

La antropometría y características de la muestra definitiva se explica en Tabla 3.

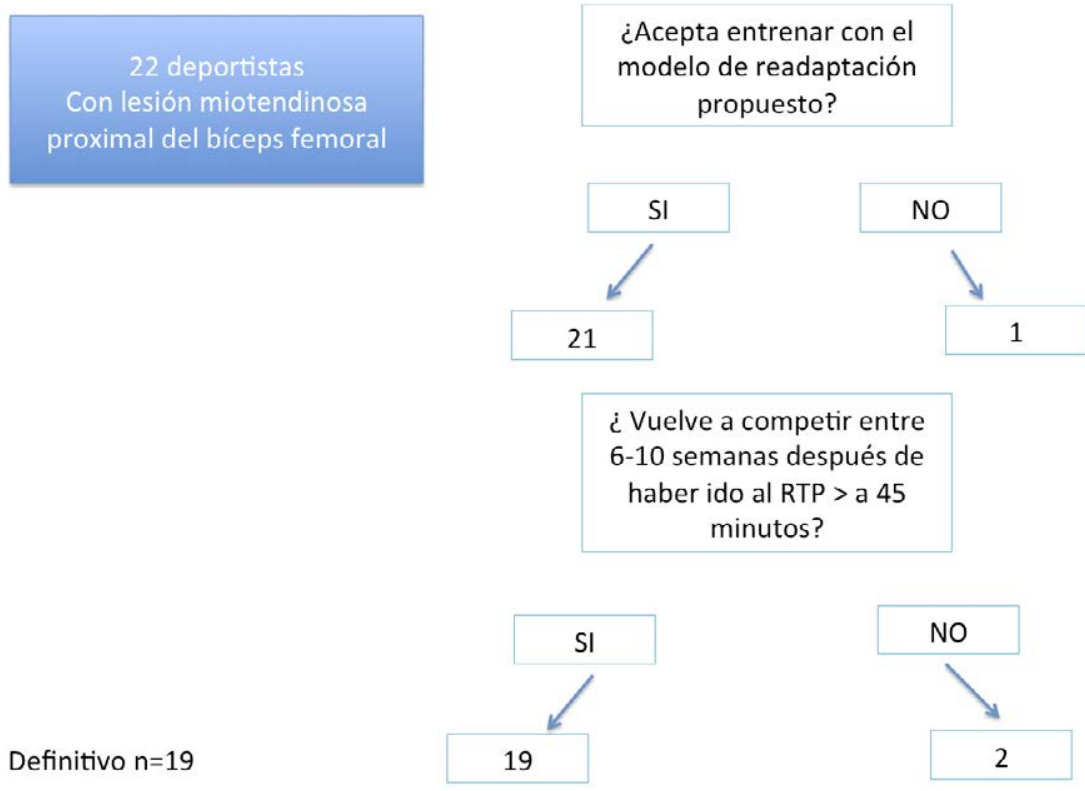

Figura 33. Representación de la muerte experimental 
Tabla 3. Información sobre los participantes $(n=19)$

\begin{tabular}{|c|c|c|c|c|c|c|}
\hline $\mathbf{N}$ & $\begin{array}{c}\text { Edad } \\
\text { (años) } \\
(\mathbf{s d})\end{array}$ & $\begin{array}{c}\text { Estatura } \\
\text { (cm) } \\
\text { (sd) }\end{array}$ & $\begin{array}{l}\text { Peso } \\
\text { (kg) } \\
\text { (sd) }\end{array}$ & $\begin{array}{c}\text { \% masa } \\
\text { grasa } \\
\text { PRE } \\
(6 \\
\text { pliegues) } \\
\text { (sd) }\end{array}$ & $\begin{array}{c}\text { Experiencia } \\
\text { profesional } \\
\text { (temporadas) } \\
\left(1^{\mathrm{a}} 02^{\mathrm{a}}\right. \\
\text { división } \\
\text { FIFA) }\end{array}$ & Nacionalidades \\
\hline 19 & $\begin{array}{c}24,23 \\
\pm \\
5,36\end{array}$ & $\begin{array}{l}179,87 \\
\pm 7,21\end{array}$ & $\begin{array}{c}74,78 \\
\pm \\
4,09\end{array}$ & $\begin{array}{c}9,96 \pm \\
0,94 \%\end{array}$ & $\begin{array}{c}4,12 \pm \\
2,34\end{array}$ & $\begin{array}{l}\text { España }(n=12), \\
\text { Alemania }(n=1) \\
\text { Serbia }(n=1) \\
\text { Brasil }(n=1) \\
\text { Uruguay }(n=2) \\
\text { Colombia }(n=1) \\
\text { Argentina }(n=1)\end{array}$ \\
\hline
\end{tabular}

\%masa grasa PRE (6 pliegues): porcentaje de masa grasa de los sujetos (toma 6 plieges Faulkner, 1968-) en estado pre-lesión; sd: standar desviation

\subsubsection{Perfil de lesión de los sujetos de la muestra}

Siguiendo criterios propuestos por diversos autores en sus clasificaciones de tejidos lesionados en cuanto afectación de la estructura (Chan et al., 2012; Mueller-Wohlfahrt et al., 2013; Valle et al., 2017), los sujetos que compusieron la muestra de esta investigación, son deportistas lesionados en grado 2 ("partial tear" y con un porcentaje superior al 5\% de fibras afectadas) donde se produjeron además de esas disrupciones de fibras, edema y hematoma (sangrado) en cara posterior del muslo, concretamente a nivel proximal en la unión miotendinosa dejando fuera del estudio (ver criterios de exclusión: apartado 3.3.3) las lesiones en vientre muscular, en cualquiera de las 5 localizaciones que define este grupo de autores (MuellerWohlfahrt et al., 2013) (intramuscular, miofascial, miofascial/perifascial, miotendinosa y combinadas) (ver figura 12). 


\subsubsection{Criterios de inclusión}

- Futbolista profesional que pertenezca a los clubes seleccionados para el estudio durante alguna o varias de las temporadas entre 2015-2018 y que sea diagnosticado de lesión proximal en isquiosurales.

- Jugador que compita al menos 45 minutos en estado sano /saludable (Prelesión) en competición en esa etapa de duración del estudio (Suárez-Arrones et al., 2015a).

- Jugador que sufra lesión miotendinosa proximal del bíceps femoral grado II, con más del $5 \%$ de fibras lesionadas, con presencia de edema y hematoma.

- Jugador que acepte entrenar durante la readaptación de su lesión miotendinosa proximal en base al programa propuesto y cumpliendo estrictamente metodología (intervención).

- Jugador que vuelva a la competición siguiendo parámetros del reentrenamiento y continue en competición a las 6-10 semanas después de ese regreso a competición postlesión (no recidiva temprana) (Fuller et al., 2006; Van der Made et al., 2017).

- Jugador que compita al menos 45 minutos en momentos post-lesión C1 y en $\mathrm{C} 2$. 


\subsubsection{Criterios de exclusión}

- Jugador que en la vuelta a la competición "return to play" no complete un mínimo de 45 minutos ó transcurran 30 o más días sin competir por diferentes motivos ajenos al proceso de readaptación (intervención).

- Jugador que, por cualquier motivo, no pueda ser registrado en competición entre 6-10 semanas (C2) tras la vuelta a competición post-lesión.

- Jugador que por motivos ajenos a los investigadores, esté sometido a otro programa durante el proceso de readaptación, o que de alguna manera alterase el modelo de intervención.

- Jugador que durante el proceso sufra cualquier otra patología que suponga la alteración del proceso.

\subsection{Procedimiento}

Para cada sujeto, se analizaron los registros en 5 momentos diferentes (figura 34), siendo el primero en estado saludable (Pre-lesión) durante un tiempo de competición (pretemporada), al igual que mostraron estudios similares (Suárez-Arrones et al., 2015a) .

El segundo registro considerado para el análisis, una vez lesionado el futbolista en union miotendinosa proximal del bíceps femoral, se registraron las variables de rendimiento durante su fase de readaptación y reentrenamiento (Jiménez-Rubio et al., 2018), cumpliendo el programa de entrenamiento compuesto por 13 ítems de dicho modelo de readaptación (Tabla 6). Previo a ese entrenamiento, los deportistas cumplían una preparación dentro de las instalaciones (denominado “indoor”) con 
tareas de fuerza especialmente a una pierna, tareas de control neuromuscular y reeducación de patrones de movimiento (Tabla 5).

El tercer registro considerado para el análisis, se realizó en entrenamiento grupal, entre la $3^{\mathrm{a}}$ y $6^{\mathrm{a}}$ sesión, sin ajustes ni adaptaciones durante el juego, en fase de entrenamiento con grupo (Ardern et al., 2016; Buckthorpe et al., 2018) mientras se preparaba entrenando para volver a la competición, y con contendios de juegos reducidos "small sided games", similar a lo mostrado por otros trabajos (Dellal et al., 2012; Gaudino et al., 2014), tareas imposibles de proponer en la fase de readaptación individual (segundo registro).

En el primer regreso a la competición $(\mathrm{C} 1)$, cuándo competía $>$ de 45 minutos y cumpliendo criterios de inclusión (ver apartado 3.3.2), se produjo el cuarto registro, idéntico momento cuantificado en otros estudios publicados (Gómez-Piqueras, González-Víllora, Sainz de Baranda Andújar, \& Contreras-Jordán, 2017; Van der Horst et al., 2016b).

Para finalizar un quinto registro $(\mathrm{C} 2)$, se realizó de nuevo en competición $(>$ de 45 minutos) en el periodo de 6-10 semanas desde esa primera competición, situación similar a estudios donde han hecho también un seguimiento tras la vuelta a la competición (Van der Made et al., 2017).

En este quinto registro, sólo se tomaron datos de una competición para cada caso, la primera que se realizaba en ese margen de 6-10 semanas tras haber vuelto a competir. 


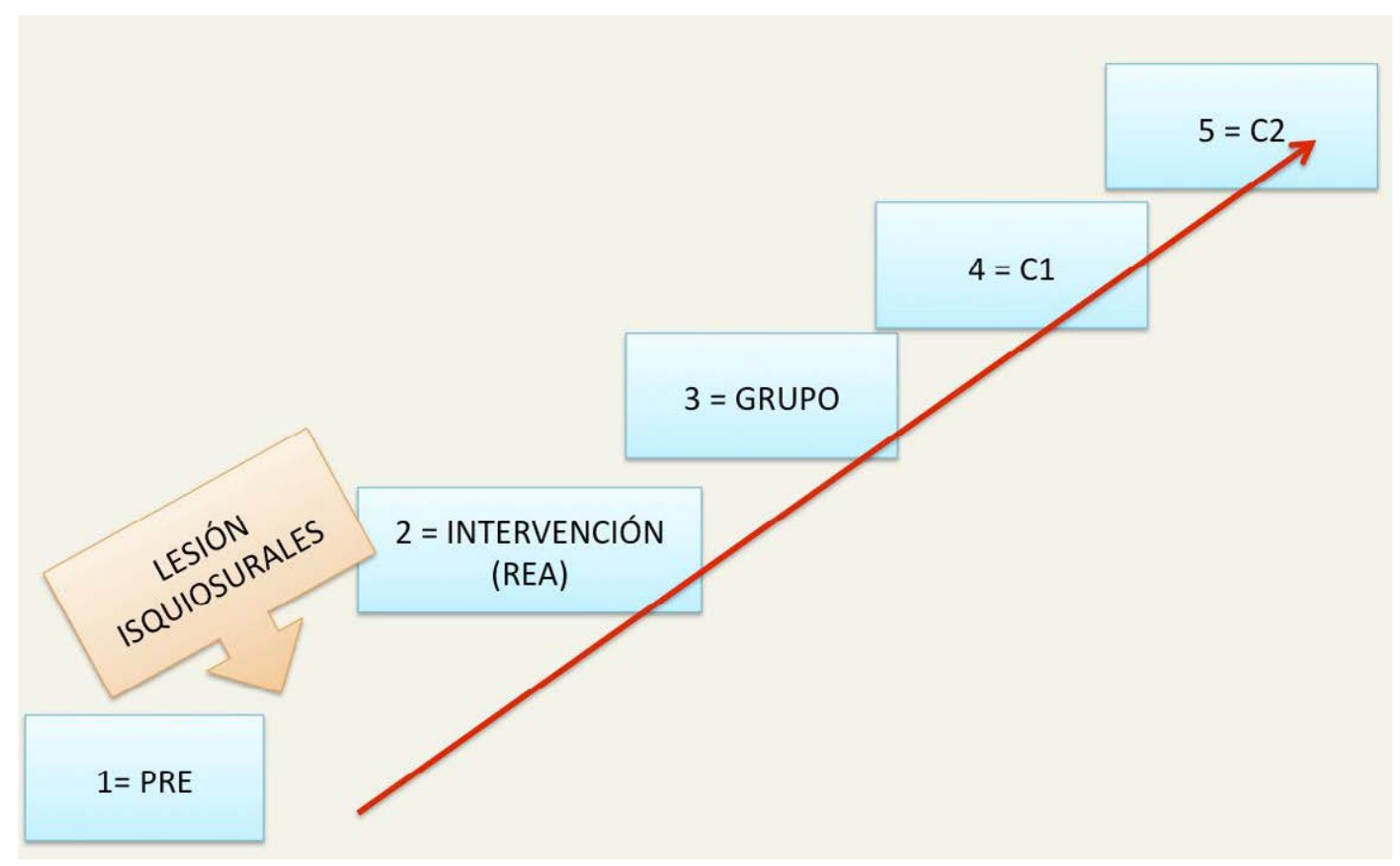

Figura 34. Cronología de los momentos donde se registraron datos

1)PRE: Pre-lesión; 2)REA: Readaptación;3) GRUPO;

4) Competición 1 (C1); 5) Competición 2 (C2)

En cada uno de los cinco momentos, los participantes se encontraron controlados por el investigador principal o colaboradores acreditados.

Tabla 4. Desarrollo de los 5 momentos donde se registraron datos

\begin{tabular}{|l|l|}
\hline PRE = Pre-lesión & $\begin{array}{l}\text { Estado saludable del deportista. Registro de } 45 \text { minutos } \\
\text { durante competición de pretemporada. }\end{array}$ \\
\hline Intervención (REA) & $\begin{array}{l}\text { Una vez producida la lesión, registro cuándo supera ítems del } \\
\text { modelo de readaptación en campo. }\end{array}$ \\
\hline GRUPO & $\begin{array}{l}\text { Registro cuándo el deportista es capaz de realizar juegos } \\
\text { reducidos con oposición. Registro entre } 3^{\text {a }} \text { y } 6^{\text {a }} \text { sesión de este } \\
\text { perfil. }\end{array}$ \\
\hline $\mathrm{C} 1$ & $\begin{array}{l}\text { Vuelta a la competición tras superar la lesión. } \\
\text { Es el "return to play". }\end{array}$ \\
\hline $\mathrm{C} 2$ & $\begin{array}{l}\text { Registro en competición que se produce entre 6-10 semanas } \\
\text { después de la primera vuelta a la competición (C1). }\end{array}$ \\
\hline
\end{tabular}


Una vez producidas las lesiones, se procedió a la explicación de los datos que se querían obtener con el fin de poder programar con mejor criterio el plan de actuación en cuanto a la recuperación.

Tras el diagnostico clínico y por imagen (a las 48 horas), se realizó un tratamiento ecoguiado con electrolisis percutánea intratisular según protocolo (Valera-Garrido et al., 2014) y se comenzó a estimular la estructura con la progresión y criterios detallados (Tabla 5).

Sobre cada jugador se obtuvieron datos mediante la herramienta GPS, colocada con chaleco individual en situaciones de seguridad para el registro de información en los momentos de readaptación (figura 20), de entrenamiento con grupo, en el retorno a la competición y en competición posterior. Ya se disponía en ese momento, de los datos del momento Pre-lesión. 


\subsection{Programa de entrenamiento durante la lesión}

\subsubsection{Tareas previas a iniciar programa definitivo}

Previo a entrar en la intervención (apartado 3.5.2) los participantes siguieron rigurosamente las tareas expuestas a continuación (Tabla 5)

Tabla 5. Entrenamiento previo a comenzar el programa de readaptación

(Adaptada de Jiménez-Rubio et al., 2018)

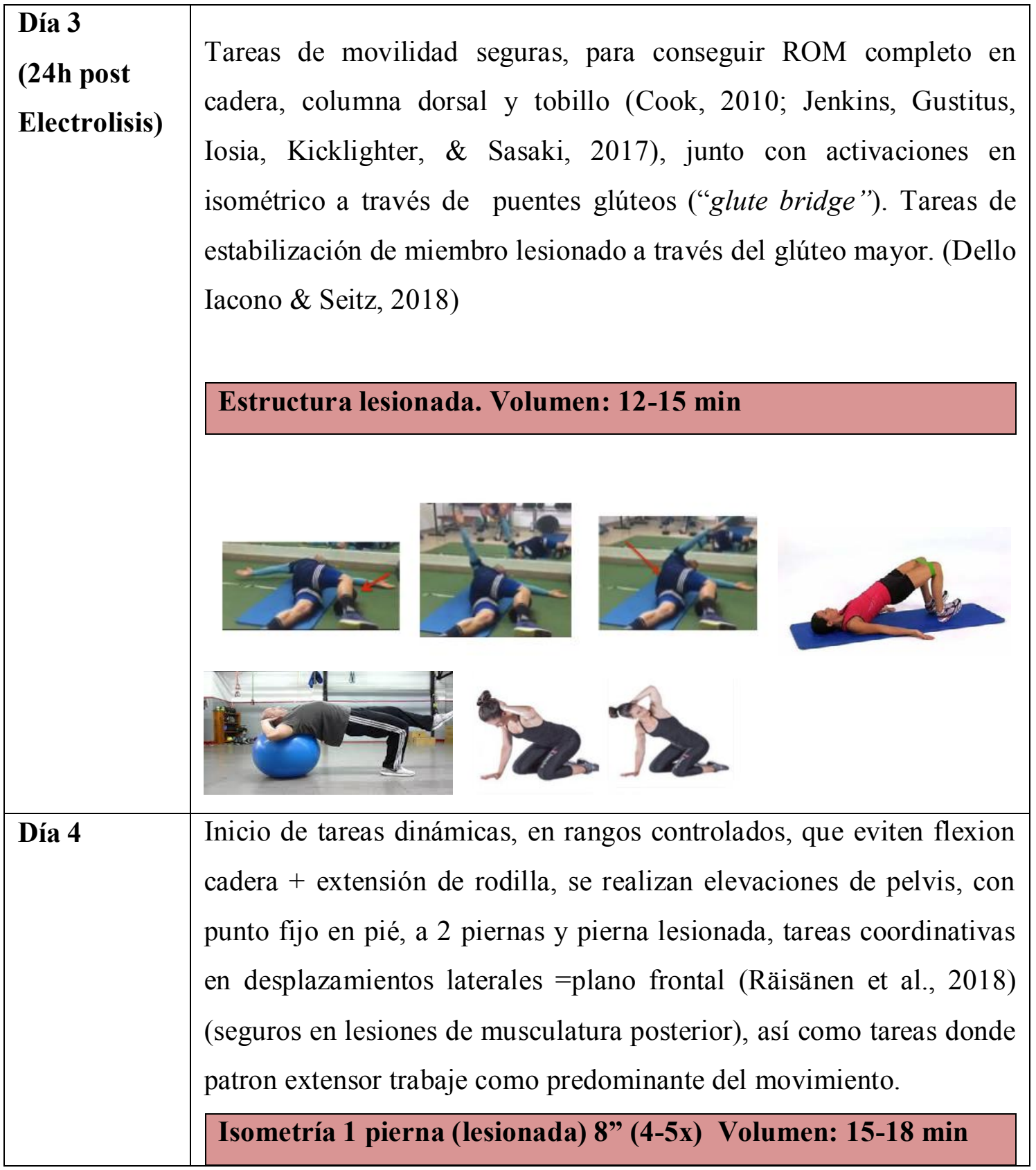




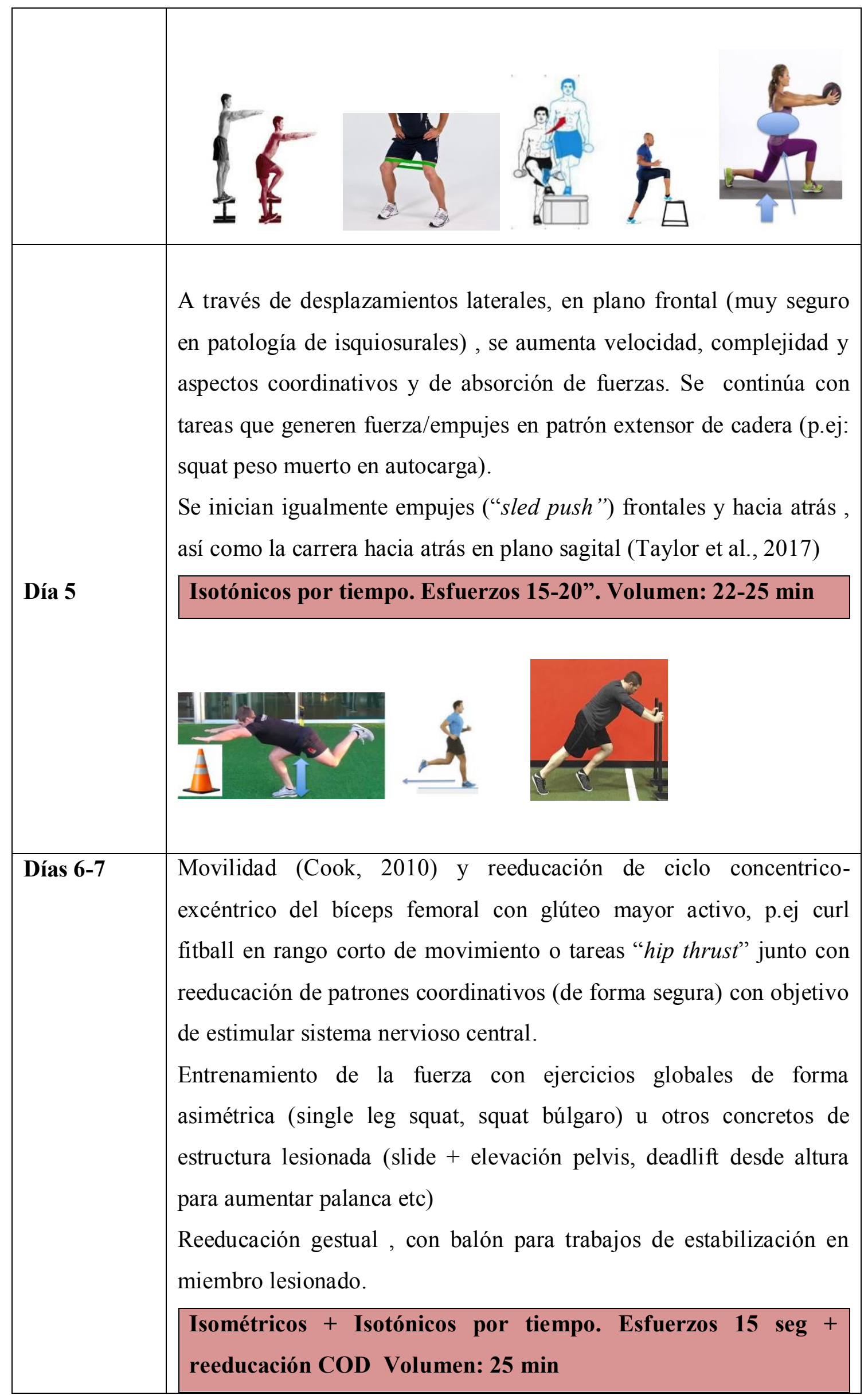




\begin{tabular}{|l|l|}
\hline & \\
\hline Días 6-8 & Tareas en interior instalación + Primeros ítems del modelo \\
\hline Días 8-16 & Superación 13 ítems del modelo \\
\hline Días 16-GRUPO & Completa ítems del modelo (Registro definitivo GPS/Intervención) \\
\hline
\end{tabular}

\subsubsection{Orden y descripción de las tareas durante la intervención del estudio}

Tabla 6. Modelo de Readaptación en campo para lesiones en la unión miotendinosa proximal del bíceps femoral (Jiménez-Rubio et al., 2018)

\begin{tabular}{|c|l|c|}
\hline No. & \multicolumn{1}{|c|}{ Descripción de las tareas } & $\begin{array}{l}\text { Tiempo } \\
\text { total }\end{array}$ \\
\hline 1 & $\begin{array}{l}\text { Activación con desplazamientos laterales y apoyos alternativos a una } \\
\text { pierna, pesos muertos y tareas controladas de aterrizajes controlando la } \\
\text { velocidad y el descenso del centro de gravedad, generando tensiones con } \\
\text { ciclo estiramiento-acortamiento de los isquiosurales (Mendiguchia et al., } \\
\text { 2017; Schuermans, Danneels, Van Tiggelen, Palmans, \& Witvrouw, } \\
\text { 2017). }\end{array}$
\end{tabular}




\begin{tabular}{|c|c|c|}
\hline 2 & $\begin{array}{l}\text { Tareas coordinativas de control motor y específicas (Anemaet \& } \\
\text { Hammerich, 2014) a nivel estático y dinámico: controles de balón, pases, } \\
\text { ciertos cambios de dirección y golpeos controlados. }\end{array}$ & $6 \mathrm{~min}$ \\
\hline 3 & $\begin{array}{l}6 \text { esfuerzos discontínuos en rango aeróbico }(<14 \mathrm{~km} / \mathrm{h}) \text { y reeducación de } \\
\text { patrones de aceleración en plano sagital }(7 \mathrm{~s} \text { de aceleración y } 3 \mathrm{~s} \text { de } \\
\text { deceleración). }\end{array}$ & $1 \min$ \\
\hline 4 & $\begin{array}{l}3 \text { series de trabajo específico de agilidad y coordinación (con y sin } \\
\text { balón en la misma tarea) en } 15 \mathrm{~m} \text { con control del movimento seguido de } \\
\text { regreso a la posición inicial (andando). }\end{array}$ & $1 \min 10 \mathrm{~s}$ \\
\hline 5 & $\begin{array}{l}3 \text { series de esfuerzo intermitente, como adaptación a tareas de alta } \\
\text { velocidad }>14 \mathrm{~km} / \mathrm{h} \text { y } 10 \mathrm{~s}<14 \mathrm{~km} / \mathrm{h} \text { en condiciones aeróbicas- } \\
\text { anaeróbicas }(70-90 \text { segundos por serie), reeducando mecánicas de } \\
\text { empujes. }\end{array}$ & $\begin{array}{l}3 \mathrm{~min} \\
30 \mathrm{~s}- \\
4 \mathrm{~min} \\
30 \mathrm{~s}\end{array}$ \\
\hline 6 & $\begin{array}{l}4 \text { series de acciones repetidas (sin incertidumbre) con cambios de } \\
\text { dirección y desaceleraciones (desplazamiento total entre } 8-14 \mathrm{~m} \text {, y tiempo } \\
\text { total inferior a } 12 \text { segundos) seguido de finalizaciones a miniporterias, } \\
\text { distantes a } 12 \mathrm{~m} \text {. Recuperación entre series: } 15 \text { segundos }\end{array}$ & $1 \min 30 \mathrm{~s}$ \\
\hline
\end{tabular}




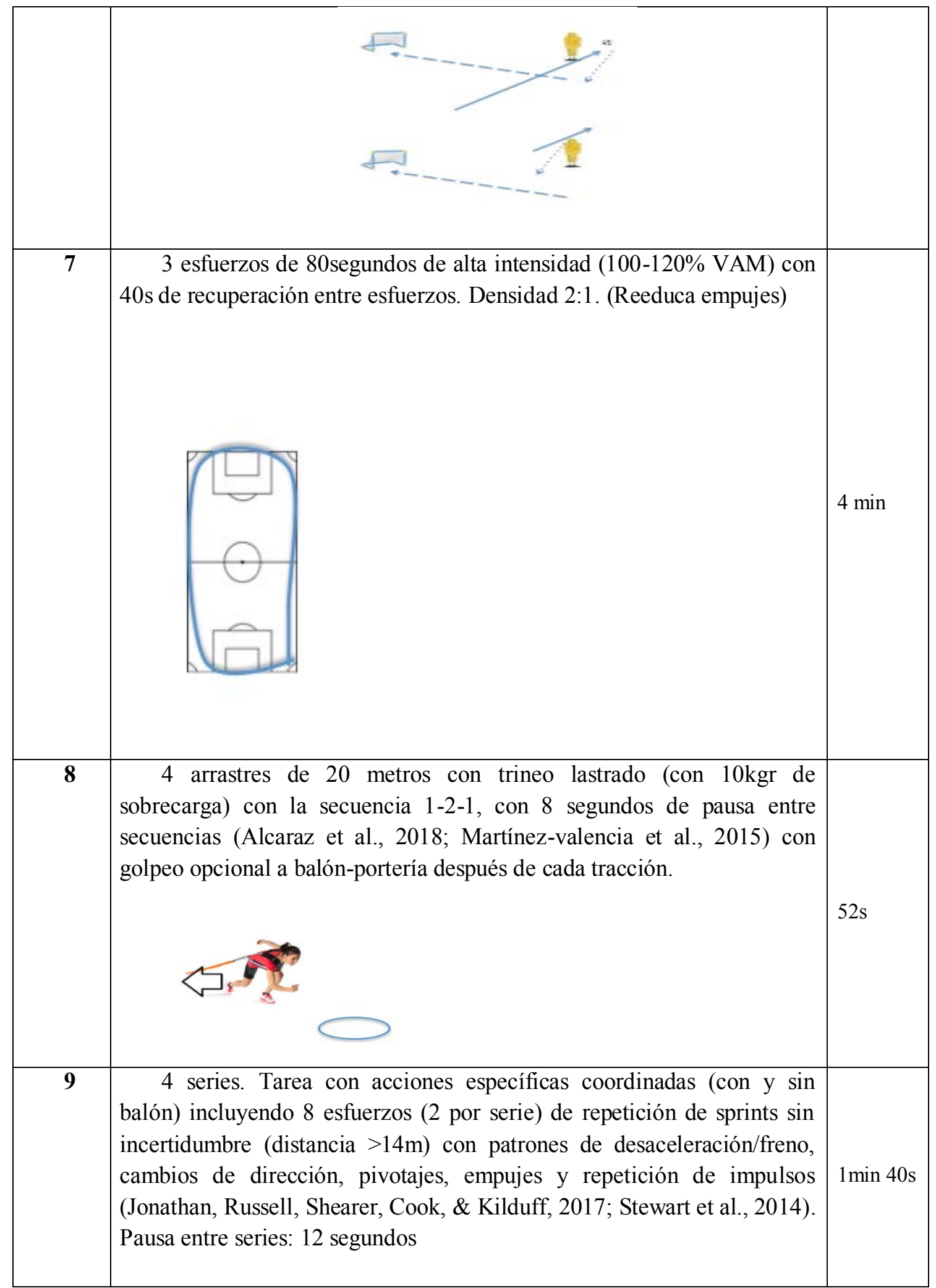




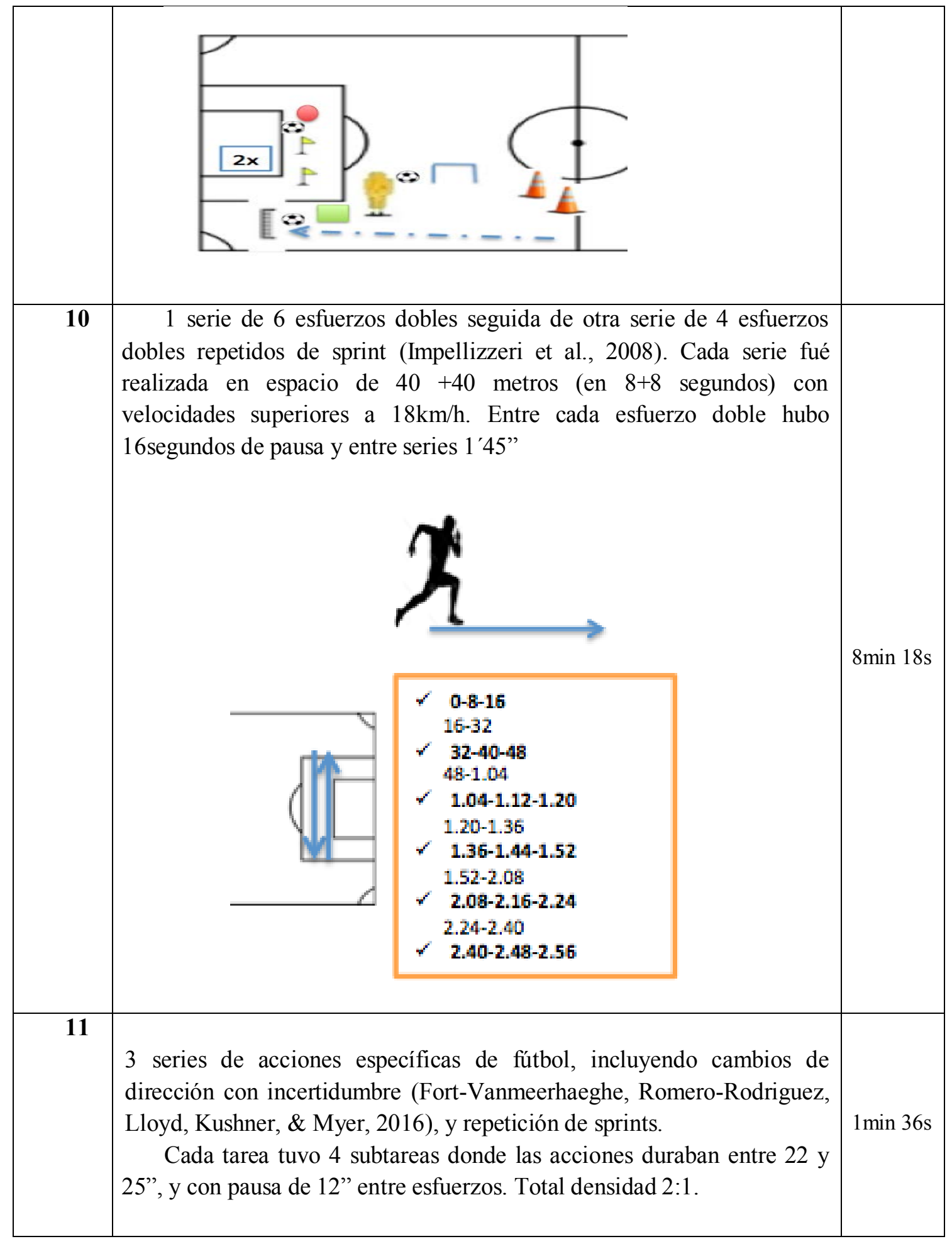




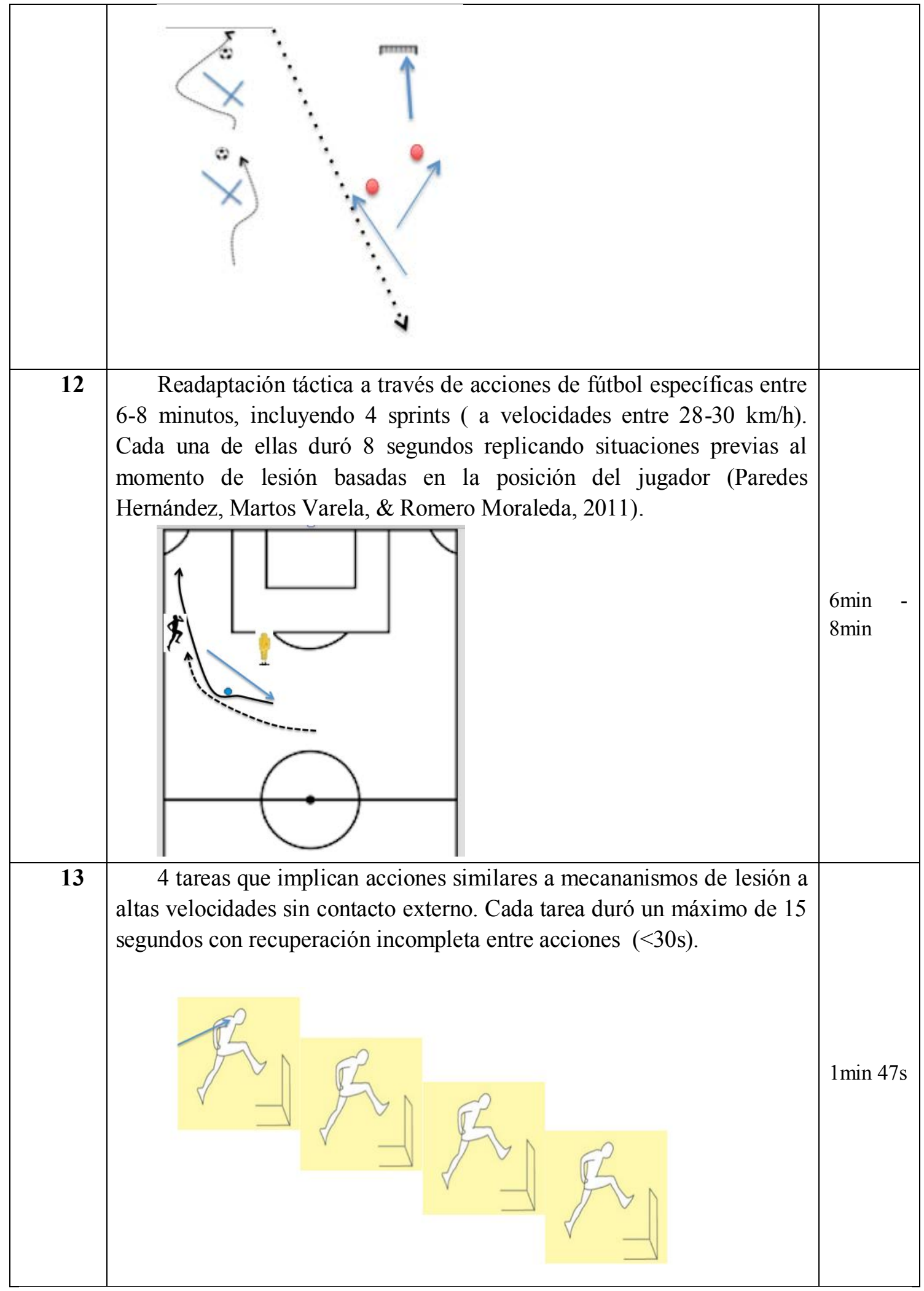


Es importante añadir también que durante el desarrollo de esta fase de readaptación, mientras los deportistas superaban ítems, realizaban un trabajo complementario consistente en entrenar movimientos pliométricos a nivel multiplanar y multidireccional en el gimnasio, con tareas de empujes, recepciones, cambios de dirección tras absorber fuerzas y otras activaciones como preparación a las tareas en campo, que fueron las evaluadas con GPS. Posterior al C1, esa progresión continuó como mecanismo protector (Gabbett \& Whiteley, 2017).

\subsection{Criterios utilizados para regresar al grupo y a la competición}

Durante el proceso, los criterios utilizados para pasar a la fase 3 (fase GRUPO) fué la de superación de tareas, concretamente los 13 ítems del modelo de readaptación y reentrenamiento (Tabla 6) sin que los deportistas manifestasen alteraciones clínicas determinantes para el servicio médico. En la literatura actual, hay pocos criterios claros y definidos en este sentido que aseguren con certeza una vuelta segura y rápida al entrenamiento colectivo tras lesiones en los isquiosurales en futbolistas profesionales (Ardern et al., 2016).

En cuanto a los criterios para pasar al momento 4 (vuelta a la competición), el denominado por otros autores el "return to performance "(Ardern et al., 2016) leemos en revisiones diferentes criterios en relación a niveles de fuerza similares, superación de tests funcionales, etc (Hickey et al., 2017; Van der Horst et al., 2017, 2016b), pero realmente se consideró la participación y superación de juegos reducidos "small sided games" con óptimo nivel de competencia entre tercera y sexta sesión grupal como se desarrolló en el apartado 3.4. 


\subsection{Validación de contenido de la intervención}

La validez es un criterio de evaluación utilizado para determinar cómo de importante es la evidencia empírica y los fundamentos teóricos que respaldan un instrumento, examen o acción realizada (Perroca, 2011). También, es entendida como el grado en que un instrumento mide lo que pretende medir o que cumple con el objetivo para el cual fué construido (Delgado-Rico, Carretero-Dios, \& Ruch, 2012). Este criterio es fundamental para considerar válida una prueba, por ello la validez junto a la fiabilidad determinan la calidad que posee un instrumento (Rodero, Molina Díaz, Fernández Guerrero, Ángel, \& Duque, 2015).

El proceso de validación es dinámico y continuo y va adquiriendo mayor relevancia a medida que se profundiza en él. La American Psichological Association (APA), en 1954, señaló 4 tipos de validez: de contenido, predictiva, concurrente y de constructo. Sin embargo, otros autores la clasifican en validez de apariencia, de contenido, de criterio y de constructo (Urrutia Egaña, Barrios Araya, Marina Gutiérrez Núñez, \& Mayorga Camus, 2014).

Por lo general esta forma de validez se ha determinado mediante la comparación sistematica de los ítems de la prueba con el dominio de contenido estudiado, este análisis es factible de ser llevado a cabo, de dos formas, la primera que consiste en estudiar de manera lógica y racional los ítem explicando el porque se incluye en la prueba; y la segunda en la cual con la ayuda de un grupo de jueces o expertos competentes y cualificados se evalúa el grado en que los reactivos concuerdan con los planteamientos del constructo e instrumento, siendo denominada esta técnica como el criterio de jueces y que en muchos casos ha sido la estrategia usada por excelencia para evaluar la validez de contenido (Aiken, 1980). 


\subsection{1 Índice de Validez $-\mathrm{V}$ de Aiken con expertos}

Para validar el modelo presentado en la tabla 6, en nuestro caso, se usó la V de Aiken, con grupo de expertos como exponíamos anteriormente.

El proceso consistió en hallar el coeficiente de validez de contenido $\mathrm{V}$ de Aiken (Aiken, 1985). Esta es una de las técnicas que permite cuantificar la relevancia de cada ítem respecto de un dominio de contenido formulado por $\mathrm{N}$ jueces. Su valor oscila desde 1 hasta 5, siendo el valor 1 indicativo de un "desacuerdo" y 5 indicativo de "muy de acuerdo" entre los jueces respecto a la puntuación de validez de los contenidos evaluados.

La interpretación del coeficiente se centra en la magnitud hallada y la determinación de la significación estadística obtenida mediante las tablas de valores críticos que él mismo publicó en 1985. La ecuación, algebraicamente modificada por (Penfield \& Giacobbi, Jr., 2004), es:

$$
V=\frac{\bar{X}-l}{K}
$$

Donde: $\mathbf{X} \overline{\text { es }}$ la media de las calificaciones de los jueces en la muestra . $\mathbf{l}$ es la calificación más baja posible. $\mathbf{k}$ es el rango de los valores posibles de la escala Likert utilizada.

Para ello, hay varios intervalos para elegir el criterio de exclusión de ítems, para ello se ofrecen niveles liberales $\mathrm{V}_{0}=0,5$ (Cicchetti, 1994) o más restrictivos como $\mathrm{V}_{0}=$ 0,70 (Charter, 2003) o más aún como $\mathrm{V}_{0}=0,80$ (Guilford, 1954)

En este estudio, valoraremos el estándar mínimo más exigente V=0,75 (Penfield \& Giacobbi, 2004).

En este sentido, los valores cuantitativos otorgados por el panel de 15 expertos fueron muy elevados, y se reflejan en la prueba V de Aiken (Tabla 7), obteniendo valores de 
0,88, 0,90 y 0,93 entre otros, para el conjunto del cuestionario, superior a los mínimos (0.75) propuestos por (Penfield \& Giacobbi, Jr., 2004).

Estos expertos, que evaluaron modelo Jiménez-Rubio et al., (2018), reunían una determinada preparación académica y alta experiencia en la readaptación de lesiones y entrenamiento con deportistas lesionados (ver Tabla 8).

Otros autores lo hicieron de forma muy similar antes (Gómez-Piqueras, GonzalezRubio, Sainz de Baranda, Najera, \& de Baranda, 2018; Zambaldi et al., 2017) evaluando igualmente con un panel de expertos y $\mathrm{V}$ de Aiken para obtener resultados en cuanto al uso de tests funcionales y así obtener información para decidir el regreso a la competición en deporte profesional. 
Tabla 7. Evaluación de ítems del modelo con $\mathrm{V}$ de Aiken.

\begin{tabular}{|c|c|c|c|c|c|c|c|}
\hline Ítem & 1 & 2 & 3 & 4 & 5 & Media & V de Aiken $(95 \% \mathrm{CI})$ \\
\hline 1 & 1 & 1 & 2 & 2 & 9 & 4.13 & $0.78(0.63-0.88)$ \\
\hline 2 & & & 3 & 2 & 10 & 4.47 & $0.87(0.73-0.94)$ \\
\hline 3 & & & 1 & 2 & 12 & 4.73 & $0.93(0.81-0.98)$ \\
\hline 4 & & & 2 & 2 & 11 & 4.60 & $0.90(0.77-0.96)$ \\
\hline 5 & & & 2 & 3 & 10 & 4.53 & $0.88(0.75-0.95)$ \\
\hline 6 & & & & 1 & 14 & 4.93 & $0.98(0.88-1.00)$ \\
\hline 7 & & & 1 & 2 & 12 & 4.73 & $0.93(0.81-0.98)$ \\
\hline 8 & & & 1 & 2 & 12 & 4.73 & $0.93(0.81-0.98)$ \\
\hline 9 & & & 1 & 1 & 13 & 4.80 & $0.95(0.83-0.99)$ \\
\hline 10 & & 1 & 1 & 3 & 10 & 4.47 & $0.87(0.73-0.94)$ \\
\hline 11 & & & & 2 & 13 & 4.87 & $0.97(0.86-0.99)$ \\
\hline 12 & & & 2 & 2 & 11 & 4.60 & $0.9(0.77-0.96)$ \\
\hline 13 & & & & 2 & 13 & 4.87 & $0.97(0.86-0.99)$ \\
\hline
\end{tabular}

Estos 15 expertos, cumplen un perfil profesional (Tabla 8) que justifica su experiencia y profesionalidad así como conocimiento del área de intervención. 
Tabla 8. Perfil académico y profesional de los expertos (15) que evaluaron modelo JiménezRubio et al.,(2018).

\begin{tabular}{|c|c|c|}
\hline \multicolumn{3}{|l|}{ Formación académica } \\
\hline Perfil de formación & Número & $\%$ \\
\hline Doctor universitario (Phd) & 8 & 53,33 \\
\hline Máster Oficial & 4 & 26,66 \\
\hline Grado (CAFYD) & 3 & 20 \\
\hline \multicolumn{3}{|l|}{ Otras formaciones académicas complementarias } \\
\hline & Número & $\%$ \\
\hline Grado en Fisioterapia & 4 & 26,6 \\
\hline Máster en Readaptación + Fisioterapeuta & 3 & 20 \\
\hline Máster en Entrenamiento personal y en Readaptación & 6 & 40 \\
\hline Sin formación complementaria & 2 & 13,33 \\
\hline \multicolumn{3}{|l|}{ Titulaciones específicas de entrenador } \\
\hline Nivel de Licencia & Número & $\%$ \\
\hline UEFA Pro & 3 & 20 \\
\hline UEFA A & 4 & 26,66 \\
\hline UEFA B & 3 & 20 \\
\hline Sin licencia UEFA (solamente preparador físico) & 5 & 33,33 \\
\hline \multicolumn{3}{|l|}{ Experiencia con jugadores lesionados de alto nivel } \\
\hline Categoría profesional & Número & $\%$ \\
\hline Como Preparador físico $(\mathrm{PF})>10$ temporadas & 2 & 13,33 \\
\hline Como Readaptador y PF $>10-15$ temporadas & 3 & 20 \\
\hline Como Readaptador $>15$ temporadas & 10 & 66,66 \\
\hline \multicolumn{3}{|l|}{ Experiencia acumulada en la carrera (Títulos) } \\
\hline Campeonatos & Temporadas & Títulos \\
\hline UEFA Champions League & 3 & 2 \\
\hline UEFA Europa League & 14 & 1 \\
\hline $1^{\mathrm{a}}$ Division & 211 & $\mid \begin{array}{lcr}5 & \text { Ligas } & +3 \\
\text { Copas del rey }\end{array}$ \\
\hline $2^{\mathrm{a}}$ División & 5 & \\
\hline
\end{tabular}




\subsection{Análisis estadístico}

Se realizó un análisis descriptivo (media \pm sd) de las 13 variables en los 3 instantes, por una parte de los momentos PRE-REA-GRUPO (datos de competición y entrenamiento) y mismo análisis de los momentos de PRE-C1-C2 (datos de competición).

Para evaluar el efecto del programa de readaptación y reentrenamiento en estas variables se desarrolló un modelo lineal mixto con el que se estimó el cambio en los parámetros evaluados en los 3 instantes citados (en ambas comparaciones).

Para dicho programa, el instante de toma de datos fué el factor fijo, y el deportista, se consideró como el factor aleatorio (Tabla 2).

Para realizar ambas comparaciones, tras el análisis descriptivo, las variables se transformaron a nivel logaritmo 100 (Hopkins, Marshall, Batterham, \& Hanin, 2009) y se realizó la prueba de Kolmogorov-Smirnov para comprobar la distribución normal de los datos. Así se constató en todas las variables.

También se compararon los valores entre los 3 instantes, con correcciones de Bonferroni. Las diferencias entre las variables, fueron comparadas con inferencias basadas en magnitudes ("magnitude based inferences") (Hopkins et al., 2009) siendo los límites para las diferencias estandarizadas como triviales $(<0.2)$, pequeñas $(0.2$ $0.6)$, moderadas (0.6-1.2), grandes $(1.2-2.0)$ y extremadamente grandes $(>2.0)$.

Las interpretaciones tras las comparaciones se realizaron utilizando este "magnitud based inferences” y el tamaño del efecto. 
El tamaño del efecto, con el intervalo de confianza del 90\% (Hopkins et al., 2009) se evaluó cualitativamente de la siguiente manera:

- cambios triviales o no determinantes (“unclear").

- mejoras/cambios poco posibles (“unlikely improvement/different”)

- posibles mejoras/cambios ("posible improvement/different")

- probables mejoras/cambios ("likely improvement/different”)

- muy probables mejoras/cambios ("very likely improvement/different")

- cambios/ mejoras casi con certeza ("almost certain improvement/different")

Todos los cálculos se realizaron con el software IMB ${ }^{\circledR}$ SPSS ${ }^{\circledR}$ Statistics (Version 20.0) y Microsoft Excel 2016.

Para todos los análisis se calcularon los intervalos de confianza con $\alpha=0,1$. 



\section{IV . - Resultados}





\subsection{Resultados a nivel de días que tardaron en recuperarse}

En el presente estudio los 19 deportistas volvieron a la competición tras haber sufrido lesión en unión miotendinosa proximal del bíceps femoral, en 22,42 22,32 días.

La vuelta normalizada al entrenamiento colectivo en este estudio fué de $16,94 \pm 1,56$ días para superar juegos reducidos (“small sided games”). 


\subsection{Resultados obtenidos tras comparaciones PRE-REA - GRUPO}

A continuación se describen los cambios con sus correspondientes porcentajes (\%) que se han dado en la comparativa entre los momentos Pre-lesión(PRE), readaptación

(REA) y el entrenamiento con el grupo (GRUPO) (tablas 10 y 11 ). 
Tabla 9. Descriptivos momentos PRE- REA - GRUPO

\begin{tabular}{|c|c|c|c|c|c|c|c|}
\hline \multirow[t]{2}{*}{$\begin{array}{l}\text { Estadísticos } \\
\text { descriptivos }^{\mathrm{a}}\end{array}$} & & \multicolumn{2}{|c|}{ Pre-lesión } & \multicolumn{2}{|l|}{ REA } & \multicolumn{2}{|l|}{ Grupo } \\
\hline & $\mathbf{N}$ & Media & SD & Media & SD & Media & SD \\
\hline V1 & 19 & 17,44 & 2,95014 & 15,39 & 6,53836 & 14,4205 & 5,68253 \\
\hline V2 & 19 & 7,24 & 2,75990 & 6,16 & 2,40995 & 5,8800 & 3,58716 \\
\hline V3 & 19 & 2,04 & 1,13391 & 0,71 & 0,57239 & 1,3579 & 1,62097 \\
\hline $\mathrm{n}^{\circ} \mathrm{RSA}$ & 19 & 0,081 & 0,046055 & 0,04737 & 0,021368 & 0,05579 & 0,028542 \\
\hline $\mathrm{n}^{\circ}$ Sprints & 19 & 0,383 & 0,18750 & 0,3653 & 0,14721 & 0,3989 & 0,12396 \\
\hline Acc1 & 19 & 0,971 & 0,31831 & 0,7153 & 0,49258 & 0,9279 & 0,33605 \\
\hline Acc2 & 19 & 0,292 & 0,19332 & 0,1626 & 0,16693 & 0,2474 & 0,21442 \\
\hline Avgspeed & 19 & 6,695 & 0,6372 & 5,884 & 0,8719 & 5,242 & 0,8815 \\
\hline Max speed & 19 & 29,48 & 1,99682 & 26,6684 & 1,52609 & 28,3368 & 2,41758 \\
\hline Max Acc & 19 & 4,27 & 0,26026 & 3,8322 & 0,4261 & 4,1847 & 0,3251 \\
\hline Impacts $>7.1 \mathrm{~g}$ & 19 & 133,73 & 68,7902 & 60,000 & 40,6926 & 114,474 & 50,6934 \\
\hline WTRR & 19 & 1,58 & 0,28106 & 1,6453 & 66715 & 1,3095 & 0,12259 \\
\hline $\begin{array}{l}\text { Explosive } \\
\text { Distance/min }\end{array}$ & 19 & 12,22 & 9,1968 & 10,4421 & 2,31008 & 1,46552 & 1,25402 \\
\hline
\end{tabular}

REA: readaptación; SD: stándar desviation; V1: metros/min entre $14.4-19,7 \mathrm{~km} / \mathrm{h}$ ) "high intensity"; V2: metros/min entre $19,8-25,1 \mathrm{~km} / \mathrm{h}$ "very high intensity": V3: metros/min a más de $25.1 \mathrm{~km} / \mathrm{h}$ "sprint": $\mathbf{n}^{\mathbf{0}} \mathbf{R S A} / \mathbf{m i n}: \mathrm{n}^{\circ}$ repeated sprints ability $/ \mathrm{min} ; \mathbf{n}^{\mathbf{0}} \mathbf{s p r i n t s} / \mathbf{m i n}>\mathbf{2 1} \mathbf{k m} / \mathbf{h}: \mathrm{n}^{\circ}$ sprints cuándo supera $21 \mathrm{~km} / \mathrm{h} ; \mathbf{A c c} 1: \mathrm{n}^{\circ}$ aceleraciones y desaceleraciones $/ \mathrm{min} 2,9-4 \mathrm{~m} / \mathrm{s} 2: \mathbf{A c c} 2$ : $\mathrm{n}^{\circ}$ de aceleraciones y desaceleraciones $/ \mathrm{min}>4 \mathrm{~m} / \mathrm{s} 2$; Avg_speed: media de velocidad $(\mathrm{km} / \mathrm{h})$; Max_speed: velocidad máxima (km/h); Max Acc: máxima aceleración realizadas; WTRR: Work to rest ratio; Impacts $>$ 7,1g: $\mathrm{n}^{\circ}$ de impactos que superan 7,1g; Explosive distance: metros/min recorridos cuándo aceleración es superior a 1,2 m/s2. 
Tabla 10. Comparación PRE-REA-GRUPO para variables de rendimiento relacionadas con la velocidad y WTRR (IC 90\%)

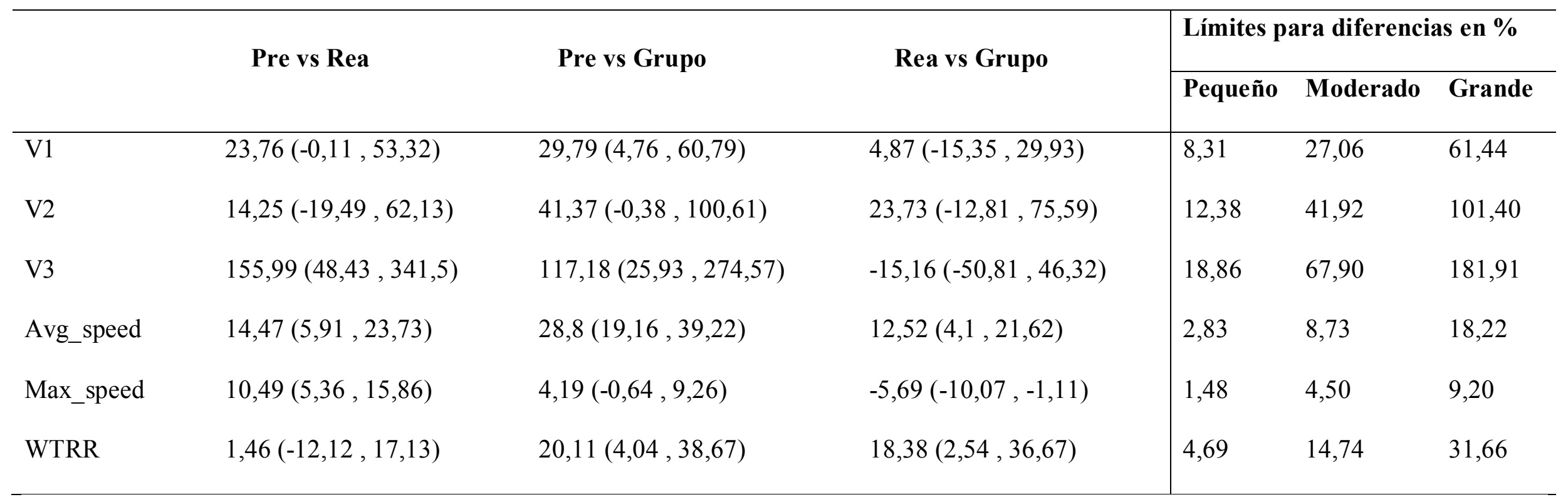

V1: \% de cambio en distancia recorrida entre 14.4 - 19,7 km/h) "high intensity"; V2: \% de cambio en distancia recorrida entre 19,8 - $25,1 \mathrm{~km} / \mathrm{h}$ "very high intensity": V3: \% de cambio en distancia recorrida a más de $25.1 \mathrm{~km} / \mathrm{h}$ "sprint": Avg_speed: \% de cambio en parámetro media de velocidad; Max_speed: \% de cambio en parámetro velocidad máxima; WTRR: \% de cambio en parámetro Work to rest ratio. 
Tabla 11. Comparación PRE-REA-GRUPO para variables de rendimiento relacionadas con aceleración, desaceleración, sprints, y explosividad (IC 90\%)

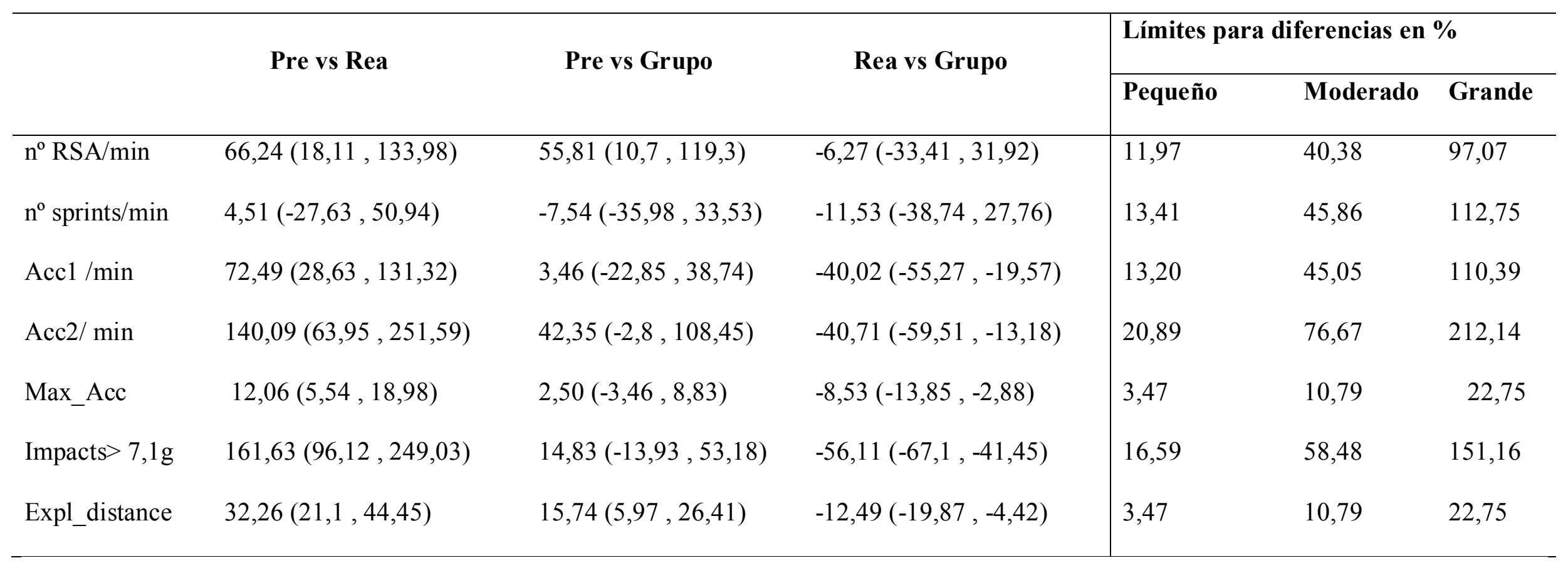

$\mathbf{n}^{0} \mathbf{R S A} / \mathbf{m i n}$ : \% de cambio en $\mathrm{m} / \mathrm{min}$ en número de "repeated sprint ability" con recuperación incompleta $(<6$ seg) de aceleración superior a $3 \mathrm{~m} / \mathrm{s} 2$; Acc1/min: \% de cambio en $\mathrm{n}^{\mathrm{o}}$ de aceleraciones y desaceleraciones entre 2,9 y $4 \mathrm{~m} / \mathrm{s} 2$ x min: Acc2/min: \% de cambio en $\mathrm{n}^{\circ}$ de aceleraciones y desaceleraciones de más de 4 m/s 2 x min ; Max_Acc \% de cambio en parámetro aceleración máxima; Expl_distance: \% de cambio en m/min del parámetro "explosive distance”, distancia recorrida cuándo aceleración es superior a 1.2 
En cuanto a la velocidad 1 (m/min entre $14.4-19,7 \mathrm{~km} / \mathrm{h})$, "high speed", se obtuvieron cambios moderados del $34,77 \%$ entre 2 registros sucesivos (IC $90 \% 27,87-43,65 \%)$.

De forma específica (Tabla 10), entre PRE y REA no se obtuvieron diferencias significativas $(p=0,103)$. El tamaño del efecto fué pequeño, (ver figura 35). Entre REA y GRUPO no se obtuvieron diferencias significativas $(p=1)$. El tamaño del efecto fué trivial (ver figura 37). Asimismo, entre PRE y GRUPO se obtuvieron diferencias significativas $(p=0,032)$. El tamaño del efecto fué moderado (ver figura 36).

$\checkmark$ En relación a la velocidad $2(\mathrm{~m} / \mathrm{min}$ entre $19,8-25,1 \mathrm{~km} / \mathrm{h})$, la denominada "Very high speed" se obtuvieron cambios moderados de $62,82 \%$ entre 2 registros sucesivos (IC 90\% 49,42-80,72\%).

De forma específica (Tabla 10), entre PRE y REA no se obtuvieron diferencias estadísticamente significativas $(p=1)$. El tamaño del efecto fué pequeño (ver figura 35). Entre REA y GRUPO no se obtuvieron diferencias significativas $(p=0,5)$. El tamaño del efecto fué pequeño (ver figura 37). Asimismo, entre momento PRE y GRUPO, no se obtuvieron diferencias significativas ( $\mathrm{p}=0,106)$. El tamaño del efecto fué pequeño (ver figura 36).

En relación a la velocidad $3(\mathrm{~m} / \mathrm{min}>25,1 \mathrm{~km} / \mathrm{h})$, la denominada "sprint" se obtuvieron cambios moderados de $113,65 \%$ entre 2 registros sucesivos (IC $90 \% 86,90-151,32 \%)$.

De forma específica (Tabla 10), entre PRE y REA se obtuvieron cambios significativos ( $p=0,002)$. El tamaño del efecto fué moderado (ver figura 35). 
Entre REA y GRUPO no se obtuvieron diferencias significativas $(p=1)$. El tamaño del efecto fué pequeño (ver figura 37). Asimismo, entre momento PRE y GRUPO, se obtuvieron diferencias significativas $(\mathrm{p}=0,010)$. El tamaño del efecto fué moderado (ver figura 36).

En relación al parámetro velocidad media (Avg_speed) realizada, se obtuvo un cambio moderado de $11,44 \%$ entre 2 registros sucesivos (IC 90\% 9,33$14,05 \%)$.

De forma específica (Tabla 10), entre PRE y REA se obtuvieron diferencias significativas ( $p=0,001)$. El tamaño del efecto fué moderado (ver figura 35). Entre REA y GRUPO se obtuvieron diferencias significativas $(p=0,006)$. El tamaño del efecto fué moderado (ver figura 37). Asimismo entre PRE y GRUPO se obtuvieron cambios significativos ( $<<0,001)$. El tamaño del efecto fué grande (ver figura 36).

$\checkmark$ En referencia a la variable pico máximo de velocidad (Max_speed), se obtuvo un cambio moderado de 6,84\% entre 2 registros sucesivos (IC 90\% $5,60-8,36 \%)$.

De forma más concreta (Tabla 10), entre PRE y REA se obtuvieron diferencias significativas $(p<0,001)$. El tamaño del efecto fué grande (ver figura 35). Entre REA y GRUPO se obtuvieron diferencias significativas $(p=0,029)$. El tamaño del efecto fué moderado (ver figura 37). Asimismo entre PRE y GRUPO, no se obtuvieron diferencias significativas $(p=0,191)$. El tamaño del efecto fué pequeño (ver figura 36). 
$\checkmark$ En cuanto al parámetro Aceleración máxima (Max_Acc) se obtuvo un cambio moderado de $13,07 \%$ entre 2 registros sucesivos (IC 90\% 10,64-16,07). De forma más concreta (Tabla 11), entre PRE y REA se obtuvieron diferencias significativas $(\mathrm{p}<0,001)$. El tamaño del efecto fué moderado (ver figura 35). Entre REA y GRUPO se obtuvieron diferencias significativas $(\mathrm{p}=0,007)$. El tamaño del efecto fué moderado (ver figura 37). Asimismo, entre PRE y GRUPO, no se obtuvieron diferencias significativas $(p=1)$. El tamaño del efecto fué trivial (ver figura 36).

$\checkmark$ Respecto al $\mathbf{n}^{\mathbf{0}} \mathbf{R S A} / \mathbf{m i n}$ con Recuperación incompleta $(<6$ seg) de ACEL $>3,0$ $\mathrm{m} / \mathrm{s} 2$, se obtuvo un cambio moderado de $60,97 \%$ entre 2 registros sucesivos (IC $90 \% 48,02-78,23 \%)$.

De forma específica (Tabla 11), entre PRE y REA se obtuvieron diferencias significativas ( $p=0,007)$. El tamaño del efecto fué moderado (ver figura 35 ). Entre el valor REA y GRUPO no se obtuvieron cambios significativos $(p=1)$. El tamaño del efecto fué moderado (ver figura 37). Asimismo entre PRE y GRUPO, se obtuvieron diferencias significativas $(p=0,020)$. El tamaño del efecto fué moderado (ver figura 36).

$\checkmark$ En cuanto al $\mathbf{n}^{\mathbf{0}}$ de sprints $>\mathbf{2 1} \mathbf{~ k m} / \mathbf{h} / \mathbf{m i n}$, se obtuvieron cambios moderados de $66,85 \%$ entre 2 registros sucesivos (IC 90\%; 50,18-82,07\%).

De forma específica (Tabla 11), entre PRE y REA, no se obtuvieron cambios significativos $(p=1)$. El tamaño del efecto fué trivial (ver figura 35). Entre REA y GRUPO no se obtuvieron cambios significativos $(p=1)$. El tamaño del efecto fué trivial (ver figura 37). Asimismo entre PRE y GRUPO, tampoco se 
obtuvieron cambios significativos $(p=1)$. El tamaño del efecto fué trivial (ver figura 36).

En cuanto a $\mathbf{n}^{\mathbf{0}}$ de aceleraciones y desaceleraciones entre 2,9 y $4 \mathrm{~m} / \mathrm{s} 2$ / min (Acc1), se obtuvieron cambios moderados de 50,49\%, entre 2 registros sucesivos (IC 90\%; 40,03-64,24\%).

De forma específica (Tabla 11), entre PRE y REA se obtuvieron diferencias significativas $(\mathrm{p}=0,001)$. El tamaño del efecto fué moderado (ver figura 35). Entre el valor REA y GRUPO se obtuvieron diferencias significativas $(p=0,001)$. El tamaño del efecto fué pequeño (ver figura 37). Asimismo entre PRE y GRUPO, no se obtuvieron cambios significativos $(p=1)$. El tamaño del efecto fué trivial (ver figura 36).

Respecto al $\mathbf{n}^{\circ}$ de aceleraciones y desaceleraciones de alta intensidad $(> \pm 4$ $\mathbf{m} / \mathbf{s}^{2} / \mathbf{m i n}$ ) (Acc_2), se obtuvieron cambios pequeños del $25,25 \%$ entre 2 registros sucesivos (IC 90\% 20,38-31,44\%).

De forma específica (Tabla 11), entre PRE y REA se obtuvieron diferencias significativas $(p<0,001)$. El tamaño del efecto fué moderado (ver figura 35). Entre REA y GRUPO, se obtuvieron diferencias significativas ( $p=0,013)$. El tamaño del efecto fué pequeño (ver figura 37). Asimismo entre PRE y GRUPO no se obtuvieron diferencias significativas $(\mathrm{p}=0,144)$. El tamaño del efecto fué pequeño (ver figura 36).

En cuanto al $\mathbf{n}^{\mathbf{0}}$ de impactos $>$ 7,1 $\mathrm{g}$ se obtuvieron cambios pequeños del $49,39 \%$ entre 2 registros sucesivos (IC 90\%; 39, 19-62,79\%).

De forma específica (Tabla 11), entre PRE y REA se obtuvieron diferencias significativas $(p<0,001)$. El tamaño del efecto fué grande (ver figura 35). Entre 
REA y GRUPO, también se obtuvieron diferencias significativas $(p<0,001)$. El tamaño del efecto fué moderado (ver figura 37). Asimismo, entre PRE y GRUPO no se obtuvieron cambios significativos $(p=0,88)$. El tamaño del efecto fué pequeño (ver figura 36).

Respecto al "work to rest ratio", se obtuvieron cambios moderados del $22,15 \%$ entre 2 registros sucesivos (IC 90\%; 17,92-27,49\%).

De forma específica (Tabla 10), entre PRE y REA no se obtuvieron cambios significativos $(p=1)$. El tamaño del efecto fué trivial (ver figura 35). Entre REA y GRUPO, se obtuvieron diferencias significativas $(p=0,040)$. El tamaño del efecto fué moderado (ver figura 37). Asimismo, entre PRE y GRUPO se obtuvieron diferencias significativas $(p=0,023)$. El tamaño del efecto fué moderado (ver figura 36).

En referencia a $\mathbf{m} / \mathbf{m i n}$ en "explosive distance" (distancia recorrida cuándo la aceleración supera 1,2 m/s2), se obtuvieron cambios moderados del 13,07\% entre 2 registros sucesivos (IC 90\%; 10,64-16,07\%).

De forma específica (Tabla 11), entre PRE y REA se obtuvieron diferencias significativas $(p<0,001)$. El tamaño del efecto fué grande (ver figura 35). Entre REA y GRUPO, también se obtuvieron diferencias significativas $(\mathrm{p}<0,001)$. El tamaño del efecto fué moderado (ver figura 37). Asimismo, entre PRE y GRUPO se obtuvieron diferencias significativas $(p=0,002)$. El tamaño del efecto fué moderado (ver figura 36).

De forma gráfica, se expone cómo fué el tamaño del efecto una vez realizadas las comparaciones entre los momentos PRE-REA (ver figura 35), PRE-GRUPO (ver figura 36) y REA-GRUPO ( ver figura 37). 
Cambios en variables de GPS entre los momentos PRE y REA

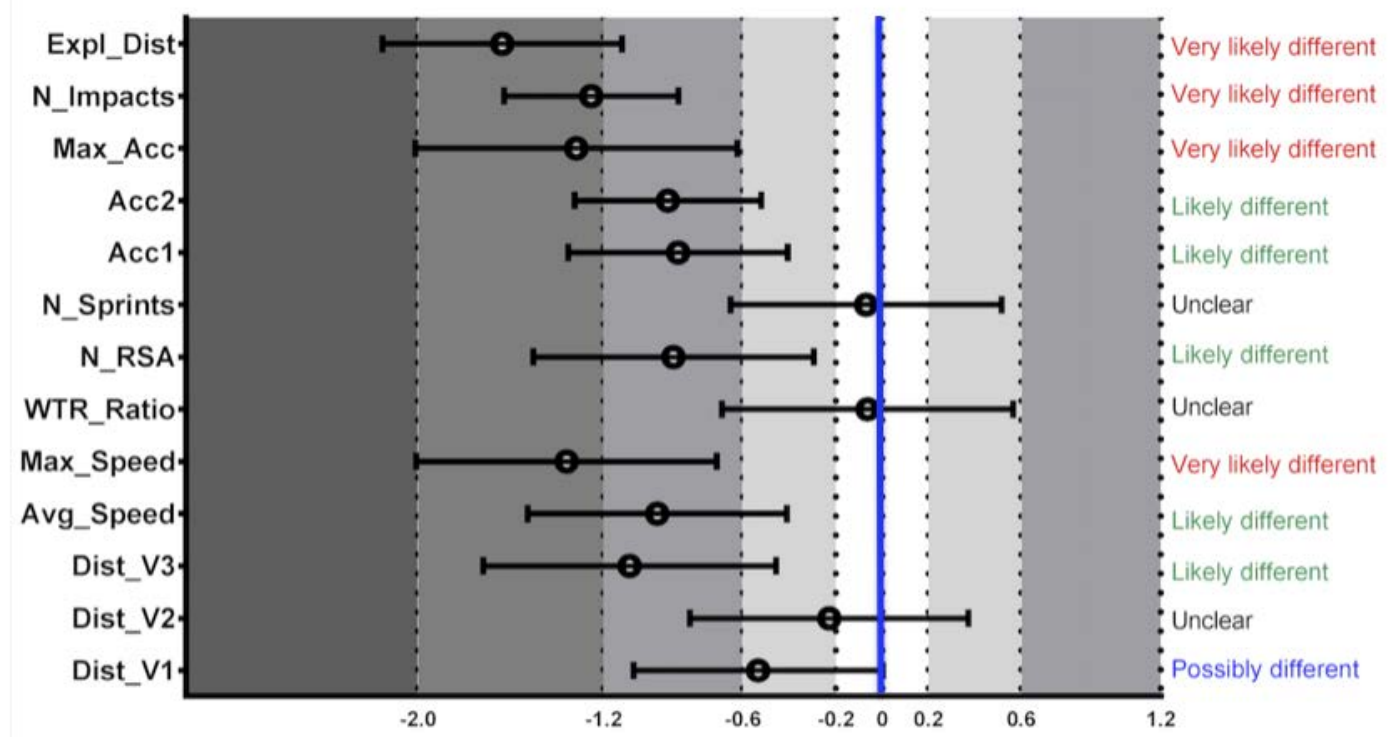

Tamaño del efecto estandarizado de Cohen (90\% IC)

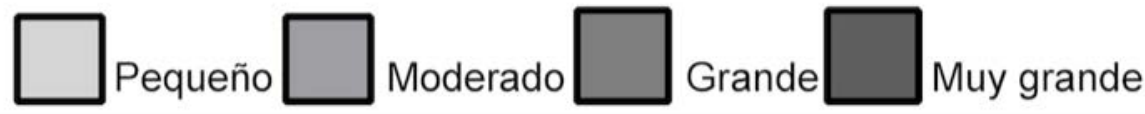

Figura 35. Diferencias de medias estandarizadas con intervalos de confianza (IC) del 90\% para cada variable relacionada con el rendimiento, entre PRE y REA

Expl Dist: metros/min recorridos cuándo aceleración es superior a $1,2 \mathrm{~m} / \mathrm{s} 2 ; \mathbf{N}^{\circ}$ Impacts: $\mathrm{n}^{\circ}$ de impactos que superan 7,1g Max Acc: máxima aceleración realizada; Acc2: $\mathrm{n}^{\circ}$ de aceleraciones y desaceleraciones $/ \mathrm{min}>4 \mathrm{~m} / \mathrm{s} 2$; Acc1: $\mathrm{n}^{\circ}$ aceleraciones y desaceleraciones $/ \min 2,9-4 \mathrm{~m} / \mathrm{s} 2: \mathbf{N}^{\circ}$ sprints: $\mathrm{n}^{\circ}$ sprints $/ \mathrm{min}$ cuándo supera $21 \mathrm{~km} / \mathrm{h} ; \mathbf{N}^{\circ}$ RSA: $\mathrm{n}^{\circ}$ repeated sprints ability $/ \mathrm{min}$ con recuperación incompleta $(<6 \mathrm{seg})$; WTR_Ratio: Work to rest ratio; Max_speed: velocidad máxima $(\mathrm{km} / \mathrm{h})$; Avg_speed: media de velocidad $(\mathrm{km} / \mathrm{h}) ;$ Dist_V3: $\operatorname{metros} / \mathrm{min}$ a más de $25.1 \mathrm{~km} / \mathrm{h}$; Dist_V2: metros $/$ min entre 19,8-25,1 km/h; Dist_V1: metros $/$ min entre $14.4-19,7 \mathrm{~km} / \mathrm{h}$ ) "high intensity" 
Cambios en variables de GPS entre los momentos PRE y GRUPO

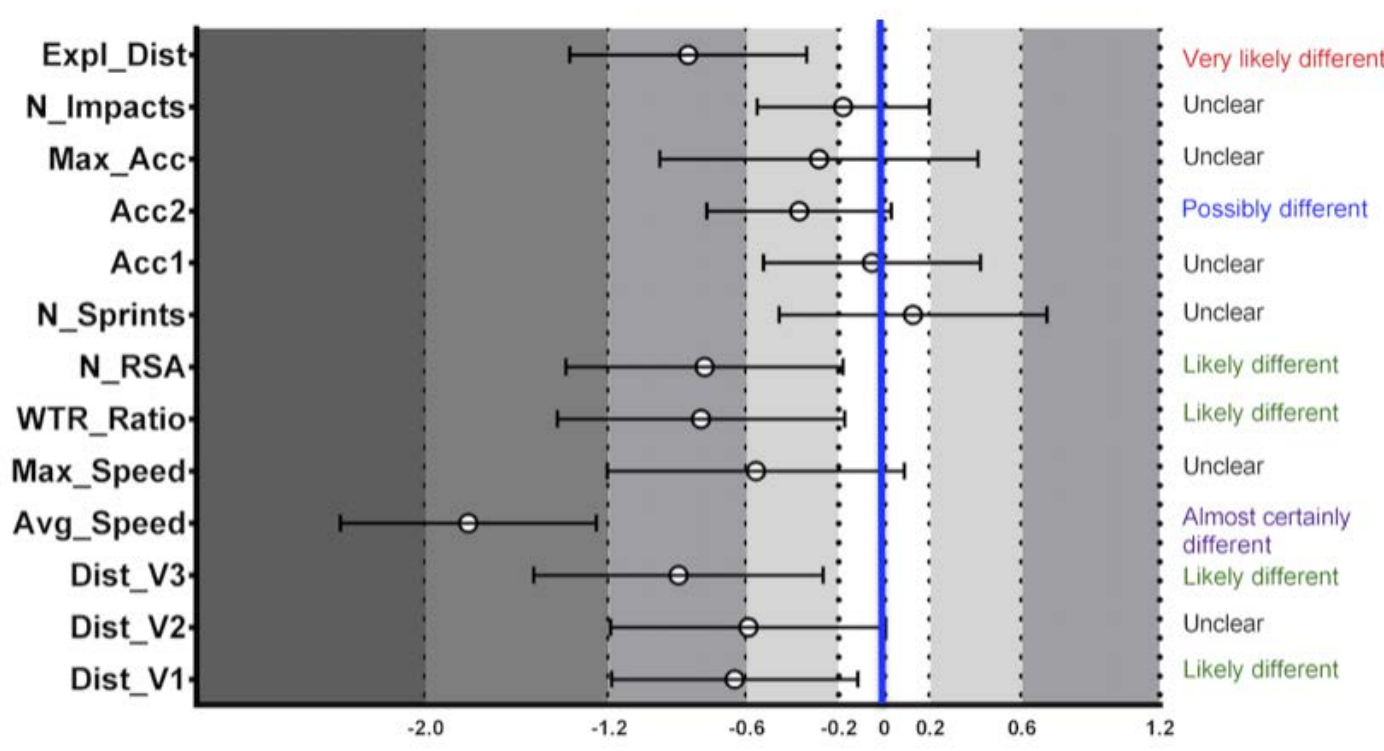

Tamaño del efecto estandarizado de Cohen (90\% IC)

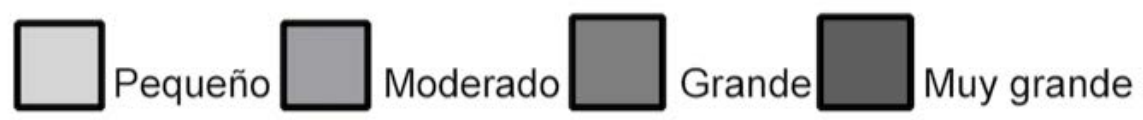

Figura 36. Diferencias de medias estandarizadas con intervalos de confianza (IC) del $90 \%$ para cada variable relacionada con el rendimiento, entre PRE y GRUPO

Expl Dist: metros/min recorridos cuándo aceleración es superior a 1,2 m/s2; $\mathbf{N}^{\mathbf{0}}$ Impacts: $\mathrm{n}^{\mathrm{o}}$ de impactos que superan 7,1g Max Acc: máxima aceleración realizada; Acc2: $\mathrm{n}^{\circ}$ de aceleraciones y desaceleraciones $/ \mathrm{min}>4 \mathrm{~m} / \mathrm{s} 2$; Acc1: $\mathrm{n}^{\mathrm{o}}$ aceleraciones y desaceleraciones $/ \min 2,9-4 \mathrm{~m} / \mathrm{s} 2: \mathbf{N}^{\mathbf{o}}$ sprints: $\mathrm{n}^{\mathrm{o}}$ sprints $/ \mathrm{min}$ cuándo supera $21 \mathrm{~km} / \mathrm{h} ; \mathbf{N}^{\mathbf{o}}$ RSA: $n^{\circ}$ repeated sprints ability /min con recuperación incompleta $(<6 \mathrm{seg})$; WTR_Ratio: Work to rest ratio; Max_speed: velocidad máxima $(\mathrm{km} / \mathrm{h})$; Avg_speed: media de velocidad $(\mathrm{km} / \mathrm{h})$; Dist_V3: $\operatorname{metros} / \mathrm{min}$ a más de $25.1 \mathrm{~km} / \mathrm{h}$; Dist_V2: metros $/$ min entre 19,8 - 25,1 km/h; Dist_V1: metros $/$ min entre $14.4-19,7 \mathrm{~km} / \mathrm{h}$ ) "high intensity" 
Cambios en variables de GPS entre los momentos REA y GRUPO

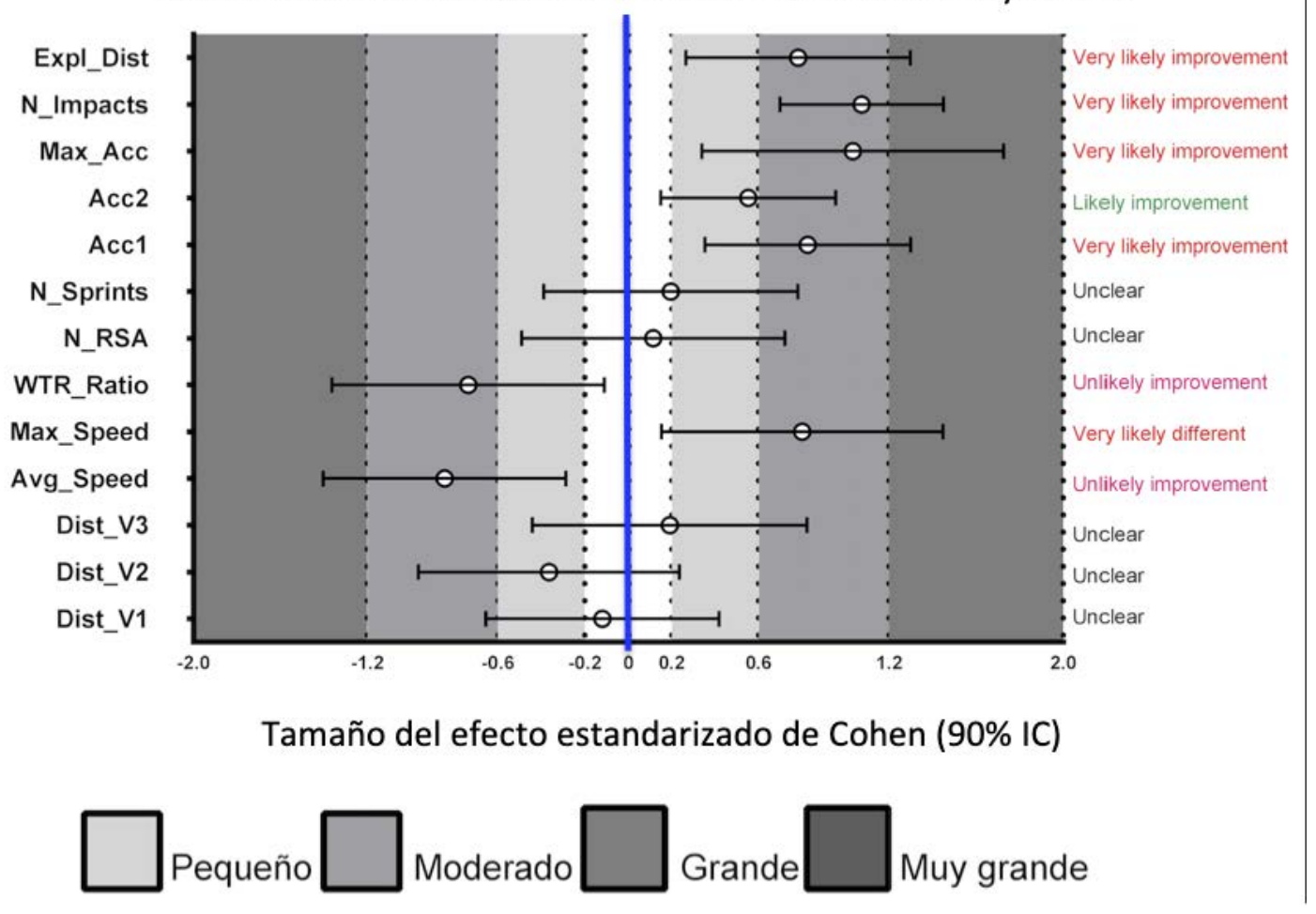

Figura 37. Diferencias de medias estandarizadas con intervalos de confianza (IC)

\author{
del $90 \%$ para cada variable relacionada con el rendimiento, entre REA y \\ GRUPO.
}

Expl Dist: metros/min recorridos cuándo aceleración es superior a $1,2 \mathrm{~m} / \mathrm{s} 2$; $\mathbf{N}^{\circ}$ Impacts: $\mathrm{n}^{\mathrm{o}}$ de impactos que superan 7,1g Max Acc: máxima aceleración realizada; Acc2: $\mathrm{n}^{\circ}$ de aceleraciones y desaceleraciones $/ \mathrm{min}>4 \mathrm{~m} / \mathrm{s} 2$; Acc1: $\mathrm{n}^{\circ}$ aceleraciones y desaceleraciones $/ \mathrm{min} 2,9-4 \mathrm{~m} / \mathrm{s} 2: \mathbf{N}^{\mathbf{o}}$ sprints: $\mathrm{n}^{\circ}$ sprints $/ \mathrm{min}$ cuándo supera $21 \mathrm{~km} / \mathrm{h} ; \mathbf{N}^{\circ}$ RSA: $\mathrm{n}^{\circ}$ repeated sprints ability /min con recuperación incompleta $(<6 \mathrm{seg})$; WTR_Ratio: Work to rest ratio; Max_speed: velocidad máxima $(\mathrm{km} / \mathrm{h})$; Avg_speed: media de velocidad $(\mathrm{km} / \mathrm{h})$; Dist_tV3: metros $/ \mathrm{min}$ a más de $25.1 \mathrm{~km} / \mathrm{h}$; Dist_V2: metros $/ \mathrm{min}$ entre 19,8-25,1 km/h; Dist_V1: metros $/$ min entre $14.4-19,7 \mathrm{~km} / \mathrm{h}$ ) "high intensity" 


\subsection{Resultados obtenidos tras comparaciones PRE-C1-C2}

A continuación se describen los cambios con sus correspondientes porcentajes (\%) que se han dado en la comparativa entre los momentos Pre-lesión (PRE), la vuelta a la competición post-lesión (C1) y el mantenimiento en la competición (C2) (tablas 13 y 14). 
Tabla 12. Descriptivos momentos Pre-lesión (PRE) y Competiciones (C1-C2)

\begin{tabular}{|c|c|c|c|c|c|c|c|}
\hline \multirow[t]{2}{*}{ Estadísticos descriptivos ${ }^{\mathrm{a}}$} & \multirow[b]{2}{*}{$\mathbf{N}$} & \multicolumn{2}{|c|}{ Pre-lesión } & \multicolumn{2}{|l|}{ C1 } & \multicolumn{2}{|l|}{$\mathrm{C} 2$} \\
\hline & & Media & SD & Media & SD & Media & SD \\
\hline V1 & 19 & 17,44 & 2,95014 & 19,34 & 4,81693 & 20,91 & 4,27709 \\
\hline V2 & 19 & 7,24 & 2,75990 & 9,29 & 3,25491 & 10,59 & 3,48570 \\
\hline V3 & 19 & 2,045 & 1,13391 & 2,98 & 1,57264 & 3,32 & 1,35591 \\
\hline $\mathrm{n}^{\circ} \mathrm{RSA} / \mathrm{min}$ & 19 & 0,081 & 0,04605 & 0,09 & 0,02674 & 0,11 & 0,03674 \\
\hline $\mathrm{n}^{\mathrm{o}}$ Sprints $/ \mathrm{min}>21 \mathrm{~km} / \mathrm{h}$ & 19 & 0,38 & 0,18750 & 0,48 & 0,12058 & 0,52 & 0,11012 \\
\hline Acc 1 & 19 & 0,97 & 0,31831 & 1,09 & 0,43701 & 1,20 & 0,36374 \\
\hline Acc2 & 19 & 0,29 & 0,19332 & 0,38 & 0,27332 & 0,45 & 0,28488 \\
\hline Avg_speed & 19 & 6,69 & 0,6372 & 6,88 & 0,6082 & 7,14 & 0,5650 \\
\hline Max_speed & 19 & 29,48 & 1,99682 & 30,87 & 1,04334 & 31,40 & 1,01436 \\
\hline Max_Acc & 19 & 4,27 & 0,26026 & 4,58 & 0,32596 & 4,72 & 0,21012 \\
\hline Impacts $>7.1 \mathrm{~g}$ & 19 & 133,73 & 68,7902 & 207,26 & 99,8186 & 222,26 & 101,6152 \\
\hline WTRR & 19 & 1,58 & 0,2811 & 1,60 & 0,3524 & 1,71 & 0,3103 \\
\hline Explosive distance/min & 19 & 12,22 & 2,3101 & 13,23 & 2,0464 & 14,27 & 1,9131 \\
\hline
\end{tabular}

C1: primera competición post-lesión; C2: competición a 6-10 semanas tras C1; SD: stándar desviation; V1: metros/min entre 14.4 - 19,7 km/h) "high intensity"; V2: metros/min entre 19,8 -

$25,1 \mathrm{~km} / \mathrm{h}$ "very high intensity": V3: metros $/ \min$ a más de $25.1 \mathrm{~km} / \mathrm{h}$ "sprint”: $\mathbf{n}^{\mathbf{0}} \mathbf{R S A} / \mathbf{m i n}: \mathrm{n}^{\mathbf{0}}$ repeated sprints ability $/ \mathbf{m i n} ; \mathbf{n}^{\mathbf{0}} \mathbf{s p r i n t s} / \mathbf{m i n}>\mathbf{2 1} \mathbf{k m} / \mathbf{h}: \mathrm{n}^{\circ}$ sprints cuándo supera $21 \mathrm{~km} / \mathrm{h} ; \mathbf{A c c 1}: \mathrm{n}^{\circ}$

aceleraciones y desaceleraciones $/ \min 2,9-4 \mathrm{~m} / \mathrm{s} 2:$ Acc2: $\mathrm{n}^{\circ}$ de aceleraciones y desaceleraciones $/ \mathrm{min}>4 \mathrm{~m} / \mathrm{s} 2 ;$ Avg_speed: media de velocidad $(\mathrm{km} / \mathrm{h}) ; \mathbf{M a x} \_\mathbf{s p e e d}:$ velocidad máxima $(\mathrm{km} / \mathrm{h})$;

Max Acc: máxima aceleración realizadas; WTRR: Work to rest ratio; Impacts > 7,1g: $\mathrm{n}^{\circ}$ de impactos que superan 7,1g; Explosive distance/min: metros/min recorridos cuándo aceleración es

superior a $1,2 \mathrm{~m} / \mathrm{s} 2$ 
Tabla 13. Comparación PRE-C1-C2 para variables de rendimiento relacionadas con la velocidad y WTRR (IC 90\%)

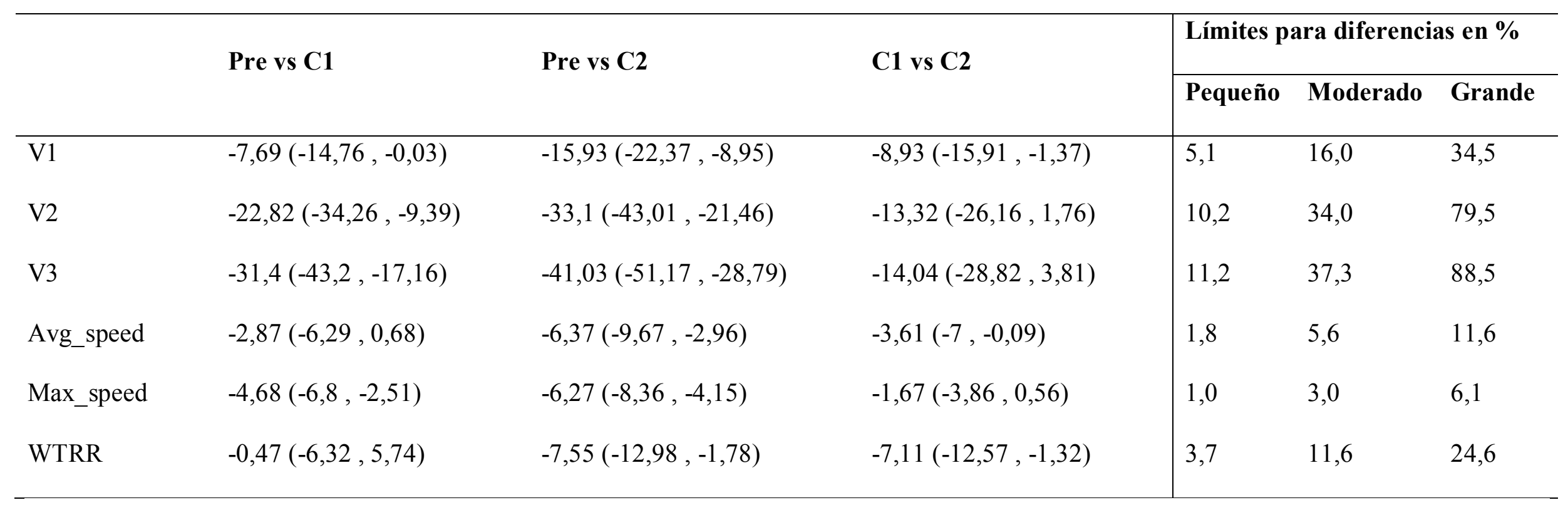

V1: \% de cambio en distancia recorrida entre $14.4-19,7 \mathrm{~km} / \mathrm{h}$ ) "high intensity"; V2: \% de cambio en distancia recorrida entre 19,8 - $25,1 \mathrm{~km} / \mathrm{h}$ "very high intensity": V3: \% de cambio en distancia recorrida a más de $25.1 \mathrm{~km} / \mathrm{h}$ "sprint": Avg_speed: \% de cambio en parámetro media de velocidad; Max_speed: \% de cambio en parámetro velocidad máxima; WTRR: \% de cambio en parámetro Work to rest ratio 
Tabla 14. Comparación PRE-C1-C2 para variables de rendimiento relacionadas con aceleración, desaceleración, sprints, y explosividad (IC 90\%)

\begin{tabular}{|c|c|c|c|c|c|c|}
\hline & Pre vs C1 & Pre vs C2 & C1 vs C2 & \multicolumn{3}{|c|}{ Límites para diferencias en \% } \\
\hline $\mathrm{n}^{\mathrm{o}}$ sprints $/ \mathrm{min}$ & $-25,94(-48,04,5,57)$ & $-31,1(-51,66,-1,8)$ & $-6,98(-34,74,32,59)$ & 12,21 & 41,27 & 99,58 \\
\hline Acc1 $1 / \min$ & $-10,4(-19,44,-0,35)$ & $-21,31(-29,25,-12,49)$ & $-12,18(-21,03,-2,33)$ & 8,83 & 28,89 & 66,12 \\
\hline Max_Acc & $-6,66(-9,83,-3,37)$ & $-9,49(-12,57,-6,3)$ & $-3,03(-6,33,0,38)$ & 1,22 & 3,7 & 7,53 \\
\hline Impacts $>7,1 \mathrm{~g}$ & $-36,7(-46,35,-25,33)$ & $-43,01(-51,69,-32,77)$ & $-9,96(-23,68,6,22)$ & 13,05 & 44,47 & 108,73 \\
\hline Expl_distance & $-8,19(-11,31,-4,97)$ & $-15,18(-18,06,-12,2)$ & $-7,61(-10,75,-4,37)$ & 3,48 & 10,81 & 22,79 \\
\hline
\end{tabular}

$\mathbf{n}^{0} \mathbf{R S A} / \mathbf{m i n}$ : \% de cambio en $\mathrm{m} / \mathrm{min}$ en número de "repeated sprint ability" con recuperación incompleta $(<6$ seg) de aceleración superior a $3 \mathrm{~m} / \mathrm{s} 2$; Acc1/min: \% de cambio en $\mathrm{n}^{\circ}$ de aceleraciones y desaceleraciones entre 2,9 y $4 \mathrm{~m} / \mathrm{s} 2$ x min: Acc2/min: \% de cambio en $\mathrm{n}^{\circ}$ de aceleraciones y desaceleraciones de más de 4 m/s2 x min ; Max_Acc $\%$ de cambio en parámetro aceleración máxima; Expl_distance: \% de cambio en $\mathrm{m} / \mathrm{min}$ del parámetro "explosive distance”, distancia recorrida cuándo aceleración es superior a $1.2 \mathrm{~m} / \mathrm{s} 2$ 
En cuanto a la velocidad 1 (m/min entre $14.4-19,7 \mathrm{~km} / \mathrm{h})$, "high intensity", se obtuvieron mejoras pequeñas de $11,74 \%$ entre 2 registros sucesivos (IC $90 \% 9,58-14,43 \%)$.

De forma específica (Tabla 13), entre PRE y C1 se encontraron diferencias estadísticamente significativas $(p=0,098)$. El tamaño del efecto fué pequeño (ver figura 38). Por otra parte, entre $\mathrm{C} 1$ y $\mathrm{C} 2$ se encontraron también diferencias significativas $(\mathrm{p}=0,041)$. El tamaño del efecto fué moderado (ver figura 40). Finalmente entre PRE y C2, se encontraron diferencias significativas ( $p=0,001)$. El tamaño del efecto fué moderado (ver figura 39).

En relación a la velocidad $2(\mathrm{~m} / \mathrm{min}$ entre $19,8-25,1 \mathrm{~km} / \mathrm{h})$, la denominada "Very high intensity" se obtuvieron mejoras pequeñas de $25,03 \%$ entre 2 registros sucesivos (IC 90\% 20,21-31,15\%).

De forma específica (Tabla 13), entre PRE y C1 se encontraron diferencias significativas ( $\mathrm{p}=0,003)$. El tamaño del efecto fué pequeño (ver figura 38). Por otra parte, entre $\mathrm{C} 1$ y $\mathrm{C} 2$ no hubo diferencias significativas $(\mathrm{p}=0,16)$. El tamaño del efecto fué pequeño (ver figura 40). Asimismo, entre PRE y C2 se encontraron diferencias significativas $(p<0,001)$. El tamaño del efecto fué pequeño, casi moderado (ver figura 39).

$\checkmark$ Respecto a la velocidad $3(\mathrm{~m} / \mathrm{min}>25,1 \mathrm{~km} / \mathrm{h})$, la denominada "sprint" se obtuvo una mejora pequeña de $30,05 \%$ entre 2 registros sucesivos (IC 90\% 24,17-37,57\%). 
De forma más concreta (Tabla 13), no se obtuvieron diferencias significativas entre PRE y C1 ( $p=0,26)$. El tamaño del efecto fué moderado (ver figura 38).

No se obtuvieron diferencias significativas entre $C 1$ y $C 2(p=0,25)$. El tamaño del efecto fué pequeño (ver figura 40). Asimismo, entre momento PRE y C2, no se obtuvieron diferencias significativas $(p=0,57)$. El tamaño del efecto fué moderado (ver figura 39).

En relación al parámetro velocidad media (Avg_speed) realizada, se obtuvo una mejora pequeña de 5,12\% entre 2 registros sucesivos (IC 90\% 4,20$6,25 \%)$.

De forma específica (Tabla 13), entre PRE y C1 no se encontraron diferencias significativas ( $p=0,24)$. El tamaño del efecto fué pequeño (ver figura 38). Por otra parte, entre $\mathrm{C} 1$ y $\mathrm{C} 2$, si se obtuvieron diferencias significativas $(\mathrm{p}=0,088)$. El tamaño del efecto fué pequeño (ver figura 40). Asimismo, entre PRE y C2 se obtuvieron diferencias significativas $(p=0,001)$. El tamaño del efecto fué moderado (ver figura 39).

En referencia a la variable pico máximo de velocidad (Max_speed), se obtuvo una mejora moderada de 3,18\% entre 2 registros sucesivos (IC 90\% $2,61-3,87 \%)$.

De forma más concreta (Tabla 13), entre PRE y C1 se obtuvieron diferencias significativas $(p<0,001)$. El tamaño del efecto fué moderado (ver figura 38). Entre $\mathrm{C} 1$ y $\mathrm{C} 2$ no se obtuvieron diferencias significativas $(\mathrm{p}=0,31)$. El tamaño del efecto fué pequeño (ver figura 40). Asimismo entre PRE y C2, se obtuvo una diferencia significativa $(p<0,001)$. El tamaño del efecto fué grande (ver figura 39). 
En relación a la aceleración máxima, se obtuvieron mejoras moderadas de 4,9\% entre 2 registros sucesivos (IC 90\%: 4,05-6,02\%).

De forma específica (Tabla 14), se obtuvieron diferencias estadísticamente significativas entre PRE y C1 $(\mathrm{p}<0,001)$. El tamaño del efecto fué moderado (ver figura 38). No se encontraron diferencias significativas entre $\mathrm{C} 1$ y $\mathrm{C} 2$ $(\mathrm{p}=0,17)$. El tamaño del efecto fué pequeño (ver figura 40). Asimismo, entre PRE y C2 no hubo diferencias significativas $(p<0,001)$. El tamaño del efecto fué grande (ver figura 39).

$\checkmark$ Respecto al $\mathbf{n}^{\mathbf{0}} \mathbf{R S A} / \mathbf{m i n}$ con Recup incompleta $(<6 \mathrm{seg})$ de Acel $>3,0 \mathrm{~m} / \mathrm{s} 2$, se obtuvo una mejora moderada de $35,71 \%$ entre 2 registros sucesivos (IC 90\% $28,60-44,87 \%)$.

De forma específica (Tabla 14), se encontraron diferencias significativas entre PRE y C1 ( $\mathrm{p}=0,056)$. El tamaño del efecto, fué moderado (ver figura 38). Por otra parte, no se obtuvieron diferencias estadísticamente significativas $(p=$ 0,45) entre C1 y C2. El tamaño del efecto, fué pequeño (ver figura 40). Asimismo entre PRE y $\mathrm{C} 2$, si se obtuvo una diferencia significativa $(\mathrm{p}=0,001)$. El tamaño del efecto, fué moderado (ver figura 39).

En cuanto al $\mathbf{n}^{\mathbf{o}}$ de Sprints $/ \mathbf{m i n}>\mathbf{2 1} \mathbf{~ k m} / \mathbf{h}$ se obtuvo una mejora moderada de $63,83 \%$ entre 2 registros sucesivos (IC 90\%; 50,18-82,07\%).

De forma específica (Tabla 14), no hubo una diferencia significativa entre PRE y C1 $(p=0,20)$. El tamaño del efecto fué pequeño (ver figura 38). Entre C1 y C2 no hubo diferencias significativas $(p=1)$. El tamaño del efecto fué trivial (ver figura 40). Asimismo entre PRE y C2, se obtuvo una diferencia significativa ( $p=0,077)$. El tamaño del efecto, fué pequeño (ver figura 39). 


\section{En cuanto a $\mathbf{n}^{\mathbf{0}}$ de aceleraciones $\mathbf{y}$ desaceleraciones de media intensidad,} entre 2,9 y $4 \mathbf{~ m} / \mathbf{s 2} / \mathbf{m i n}(A c c 1)$, se obtuvo una mejora pequeña de 15,96\%, entre 2 registros sucesivos (IC 90\%; 12,97-19,69\%).

De forma específica (Tabla 14), se obtuvieron diferencias significativas entre PRE y C1 $(p=0,085)$. El tamaño del efecto fué pequeño (ver figura 38). Se obtuvieron diferencias significativas entre $\mathrm{C} 1$ y $\mathrm{C} 2(\mathrm{p}=0,031)$. El tamaño del efecto, fué pequeño (ver figura 40). Asimismo entre PRE y C2, se obtuvo también una diferencia significativa $(\mathrm{p}<0,001)$. El tamaño del efecto,fué grande (ver figura 39).

$\checkmark$ Respecto al $\mathbf{n}^{0}$ de aceleraciones y desaceleraciones de alta intensidad $(> \pm 4$ $\mathbf{m} / \mathbf{s}^{2} / \mathbf{m i n}$ ) (Acc2), se obtuvo una mejora pequeña del 25,25\% entre 2 registros sucesivos (IC 90\% 20,38-31,44\%).

De forma específica (Tabla 14), se obtuvo una diferencia estadísticamente significativa entre PRE y $C 1 \quad(p=0,011)$. El tamaño del efecto fué pequeño (ver figura 38). Asimismo, entre $\mathrm{C} 1$ y $\mathrm{C} 2$, se obtuvieron diferencias significativas ( $p=0,011)$. El tamaño del efecto fué pequeño (ver figura 40). Asimismo entre PRE y $\mathrm{C} 2$ hubo diferencias significativas $(\mathrm{p}<0,001)$. El tamaño del efecto fué pequeño(ver figura 39).

$\checkmark$ En cuanto al $\mathbf{n}^{0}$ de impactos $>$ 7,1 $\mathrm{g}$ se obtuvo una mejora pequeña del $25,89 \%$ entre 2 registros sucesivos (IC 90\%; 20,89-32,25).

De forma específica (Tabla 14), entre PRE y C1, se obtuvo una diferencia estadísticamente significativa $(\mathrm{p}<0,001)$. El tamaño del efecto fué pequeño (ver figura 38). Entre $\mathrm{C} 1$ y $\mathrm{C} 2$, no se obtuvo una diferencia significativa $(p=0,5)$. El tamaño del efecto fué trivial (ver figura 40). Asimismo entre PRE 
y $\mathrm{C} 2$ se obtuvo una diferencia significativa $(\mathrm{p}<0,001)$. El tamaño del efecto fué moderado (ver figura 39).

$\checkmark$ Respecto al "work to rest ratio", se obtuvo una mejora pequeña del $8,80 \%$ entre 2 registros sucesivos (IC 90\%; 7,19-10,78\%).

De forma específica (Tabla 13), entre PRE y C1, no hubo diferencias estadísticamente significativas $(p=1)$. El tamaño del efecto fué trivial (ver figura 38). Entre el valor $\mathrm{C} 1$ y $\mathrm{C} 2$, hubo diferencias significativas $(\mathrm{p}=0,032)$. El tamaño del efecto fué pequeño (ver figura 40). Asimismo entre PRE y C2 hubo diferencias significativas $(\mathrm{p}=0,020)$. El tamaño del efecto fué pequeño (ver figura 39).

En referencia a m/min en "explosive distance" (distancia recorrida cuándo la aceleración supera $\mathbf{1 , 2} \mathbf{~ m} / \mathbf{s} 2$ ), se obtuvo una mejora pequeña del 4,92\% entre 2 registros sucesivos (IC 90\%; 4,04-6,01\%).

De forma específica (Tabla 14), entre PRE y C1 hubo diferencias significativas $(p<0,001)$. El tamaño del efecto fué pequeño, casi moderado (ver figura 38). Entre $\mathrm{C} 1$ y $\mathrm{C} 2$ hubo diferencias estadísticamente significativas $(p<0,001)$. El tamaño del efecto fué pequeño (ver figura 40). Asimismo, entre PRE y C2 se obtuvieron diferencias significativas $(\mathrm{p}<0,001)$. El tamaño del efecto fué moderado (ver figura 39).

De forma gráfica, se expone cómo fueron los efectos una vez realizadas las comparaciones entre los momentos PRE-C1 (figura 38), PRE-C2 (figura 39) y C1 -C2 (figura 40). 


\section{Cambios en variables de GPS entre los momentos PRE y C1}

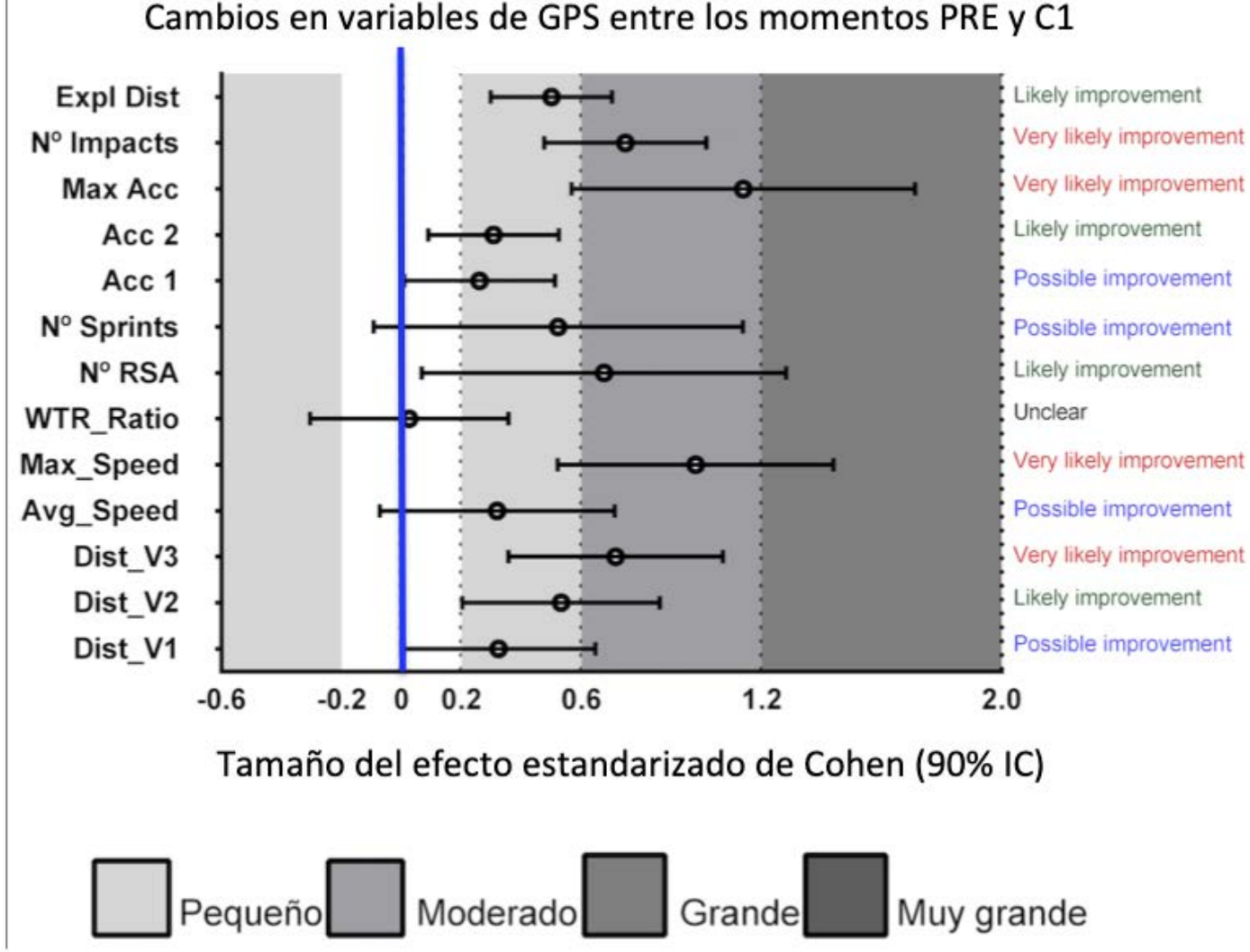

Figura 38. Diferencias de medias estandarizadas con intervalos de confianza (IC) del $90 \%$ para cada variable relacionada con el rendimiento, entre PRE y C1

Expl Dist: metros/min recorridos cuándo aceleración es superior a $1,2 \mathrm{~m} / \mathrm{s} 2$; $\mathbf{N}^{\mathbf{0}}$ Impacts: $\mathrm{n}^{\mathrm{o}}$ de impactos que superan 7,1g Max Acc: máxima aceleración realizada; Acc2: $\mathrm{n}^{\circ}$ de aceleraciones y desaceleraciones $/ \mathrm{min}>4 \mathrm{~m} / \mathrm{s} 2$; Acc1: $\mathrm{n}^{\circ}$ aceleraciones y desaceleraciones $/ \mathrm{min} 2,9-4 \mathrm{~m} / \mathrm{s} 2: \mathbf{N}^{0}$ sprints: $\mathrm{n}^{\mathrm{o}}$ sprints $/ \mathrm{min}$ cuándo supera $21 \mathrm{~km} / \mathrm{h} ; \mathbf{N}^{\circ}$ RSA: $n^{\circ}$ repeated sprints ability /min con recuperación incompleta $(<6 \mathrm{seg})$; WTR_Ratio: Work to rest ratio; Max_speed: velocidad máxima $(\mathrm{km} / \mathrm{h})$; Avg_speed: media de velocidad $(\mathrm{km} / \mathrm{h})$; Dist_V3: metros $/ \mathrm{min}$ a más de $25.1 \mathrm{~km} / \mathrm{h}$; Dist_V2: metros/min entre 19,8-25,1 km/h; Dist_V1: metros/min entre $14.4-19,7 \mathrm{~km} / \mathrm{h}$ ) "high intensity" 
Cambios en variables de GPS entre los momentos PRE y C2

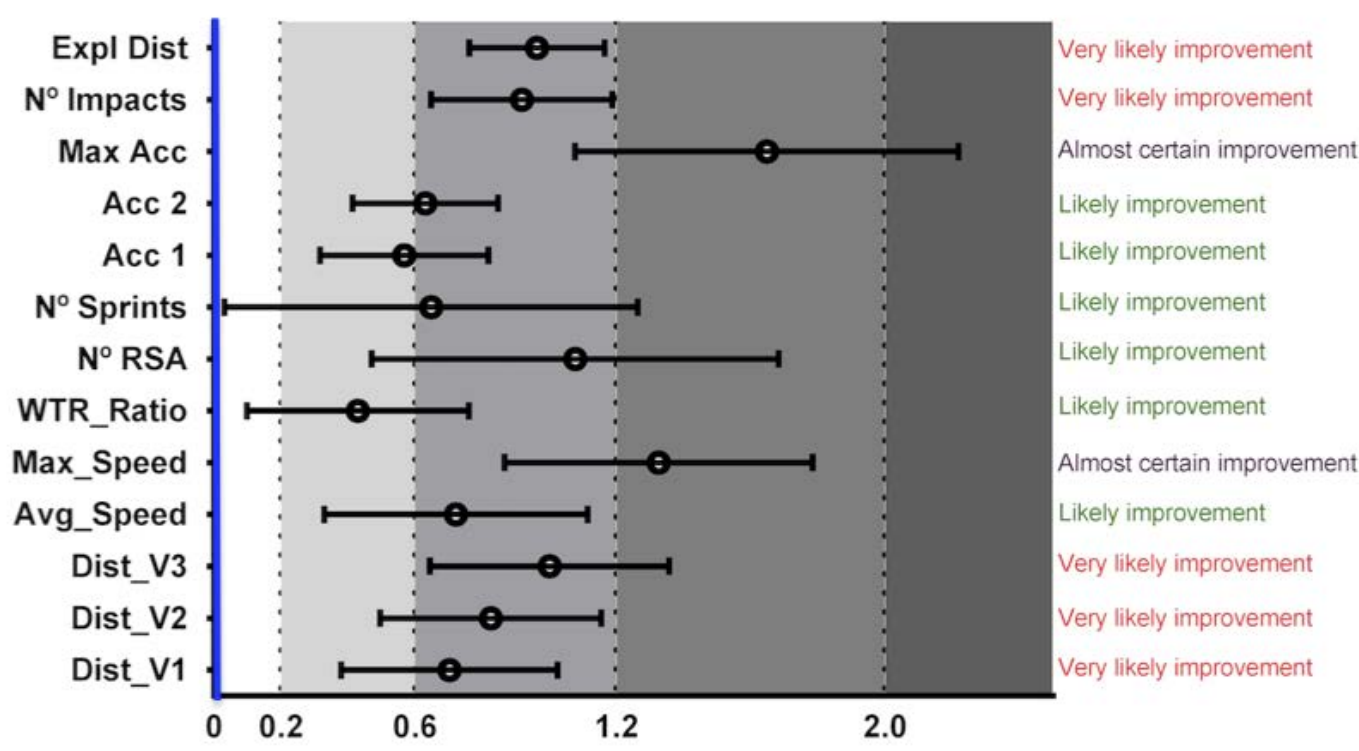

Tamaño del efecto estandarizado de Cohen (90\% IC)

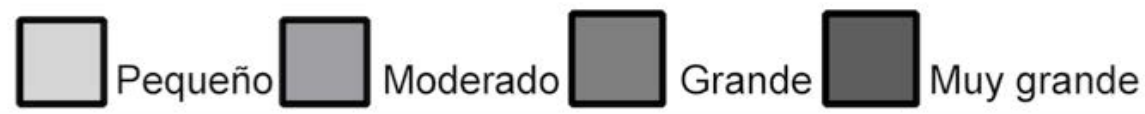

Figura 39. Diferencias de medias estandarizadas con intervalos de confianza (IC) del $90 \%$ para cada variable relacionada con el rendimiento, entre PRE y C2

Expl Dist: $\operatorname{metros} /$ min recorridos cuándo aceleración es superior a $1,2 \mathrm{~m} / \mathrm{s} 2$; $\mathbf{N}^{\circ}$ Impacts: $\mathrm{n}^{\circ}$ de impactos que superan 7,1g Max Acc: máxima aceleración realizada; Acc2: $\mathrm{n}^{\circ}$ de aceleraciones y desaceleraciones $/ \mathrm{min}>4 \mathrm{~m} / \mathrm{s} 2$; Acc1: $\mathrm{n}^{\mathrm{o}}$ aceleraciones y desaceleraciones $/ \mathrm{min} 2,9-4 \mathrm{~m} / \mathrm{s} 2: \mathbf{N}^{\mathbf{0}}$ sprints: $\mathrm{n}^{\mathrm{o}}$ sprints $/ \mathrm{min}$ cuándo supera $21 \mathrm{~km} / \mathrm{h} ; \mathbf{N}^{\mathrm{o}}$ RSA: $\mathrm{n}^{\circ}$ repeated sprints ability $/ \mathrm{min}$ con recuperación incompleta $(<6 \mathrm{seg})$; WTR_Ratio: Work to rest ratio; Max_speed: velocidad máxima $(\mathrm{km} / \mathrm{h})$; Avg_speed: media de velocidad $(\mathrm{km} / \mathrm{h})$; Dist_V3: metros $/ \mathrm{min}$ a más de $25.1 \mathrm{~km} / \mathrm{h}$; Dist_V2: metros/min entre $19,8-25,1 \mathrm{~km} / \mathrm{h}$; Dist_V1: metros $/$ min entre $14.4-19,7 \mathrm{~km} / \mathrm{h}$ ) "high intensity" 


\section{Cambios en variables de GPS entre los momentos C1 y C2}

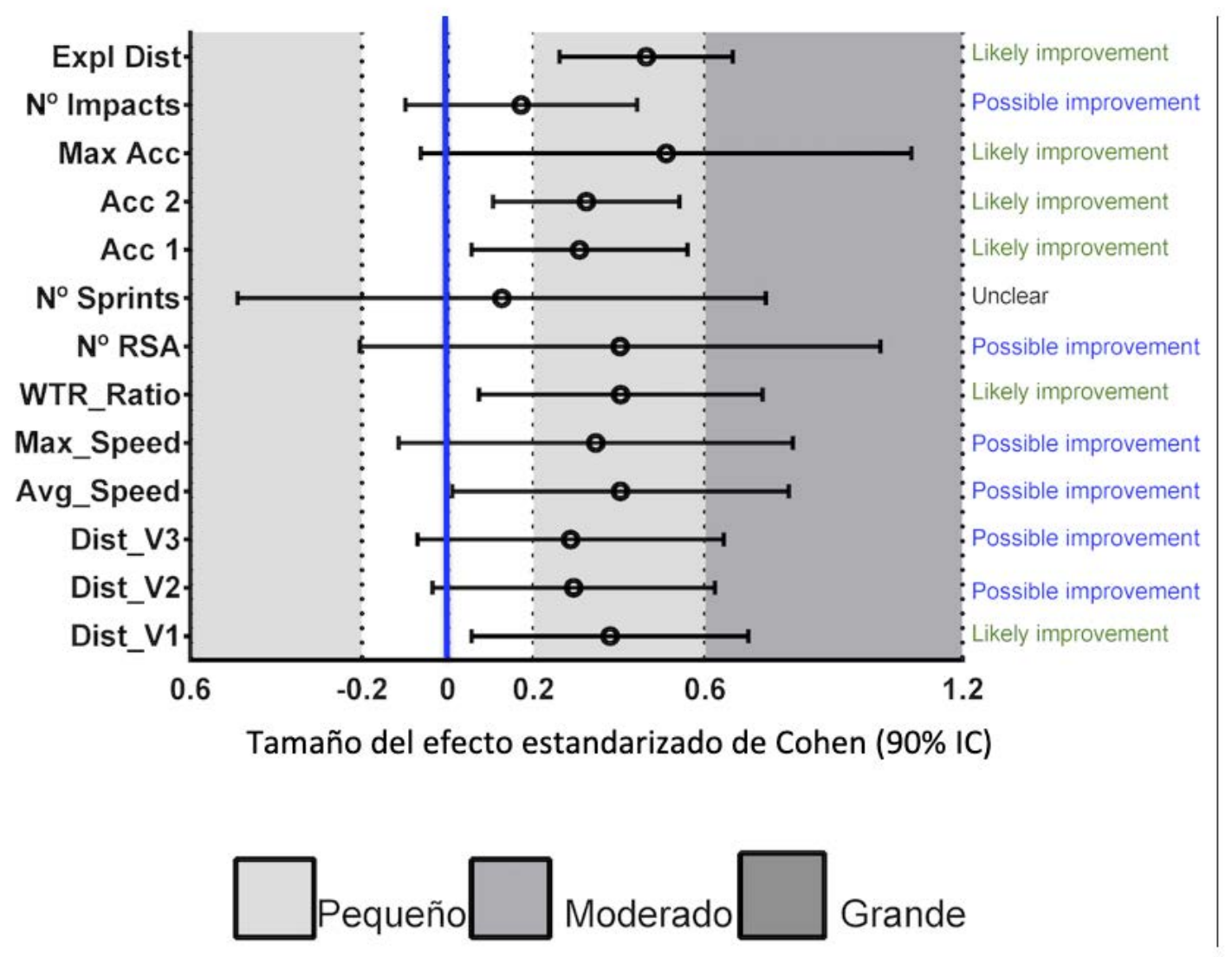

Figura 40. Diferencias de medias estandarizadas con intervalos de confianza (IC) del $90 \%$ para cada variable relacionada con el rendimiento, entre $\mathrm{C1}$ y $\mathbf{C 2}$.

Expl Dist: $\operatorname{metros} /$ min recorridos cuándo aceleración es superior a $1,2 \mathrm{~m} / \mathrm{s} 2 ; \mathbf{N}^{\circ}$ Impacts: $\mathrm{n}^{\circ}$ de impactos que superan 7,1g Max Acc: máxima aceleración realizada; Acc2: $\mathrm{n}^{\circ}$ de aceleraciones y desaceleraciones $/ \mathrm{min}>4 \mathrm{~m} / \mathrm{s} 2$; Acc1: $\mathrm{n}^{\circ}$ aceleraciones y desaceleraciones $/ \min 2,9-4 \mathrm{~m} / \mathrm{s} 2: \mathbf{N}^{\mathbf{o}}$ sprints: $\mathrm{n}^{\circ}$ sprints $/$ min cuándo supera $21 \mathrm{~km} / \mathrm{h} ; \mathbf{N}^{\circ}$ RSA: $\mathrm{n}^{\circ}$ repeated sprints ability $/ \mathrm{min}$ con recuperación incompleta $(<6 \mathrm{seg})$; WTR_Ratio: Work to rest ratio; Max_speed: velocidad máxima $(\mathrm{km} / \mathrm{h})$; Avg_speed: media de velocidad $(\mathrm{km} / \mathrm{h})$; Dist_V3: $\operatorname{metros} / \mathrm{min}$ a más de $25.1 \mathrm{~km} / \mathrm{h}$; Dist_V2: metros $/ \mathrm{min}$ entre 19,8-25,1 km/h; Dist_V1: metros $/ \mathrm{min}$ entre $14.4-19,7 \mathrm{~km} / \mathrm{h}$ ) "high intensity" 
V. Discusión 



\subsection{Discusión sobre resultado de días que tardaron en recuperarse.}

En primer lugar, es importante destacar la media de días que tardaron los deportistas en regresar a la competición oficial tras superar la lesión $(22,42 \pm 2,32$ días $)$. Se trata de un valor, dentro del rango de lo obtenido en trabajos muy actuales (Van der Made et al., 2017) donde se registraron momentos de vuelta a la competición en lesiones de isquiosurales, en diferentes deportes como balonmano, fútbol, voleibol, hockey, decathlon etc. Van der Made et al. (2017) y donde se mostraron los días que tardaron en volver a competir tras lesionarse isquiosurales con diferentes rangos de afectaciones estructurales - menos del 50\% de afectación de la sección transversal

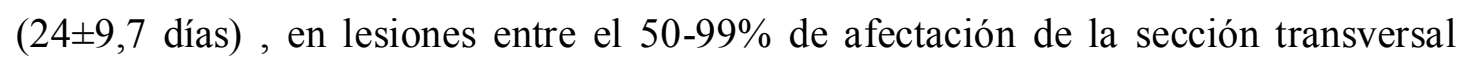
(25,3 $\pm 8,6$ días) y en lesiones con el $100 \%$ de afectación a nivel de la sección transversal (31,6 $\pm 10,9$ días) (ver figura 41$)$.

Para interpretar el resultado obtenido a nivel de días en que los deportistas lesionados regresaron al juego oficial $(22,42 \pm 2,32$ días), es necesario ser conocedor de que en ocasiones el deportista está disponible para la competición algunos días antes, pero la competición tiene unas fechas marcadas por el calendario, y es por ello por lo que la vuelta a la competición, el "return to play", se considera que no evalúa con objetividad la celeridad, precisión y calidad de la recuperación.

Es conocido también situaciones de otro tipo de casos, donde deportistas que están listos tras una lesión, no regresan a la competición por decisiones técnicas o por otros motivos (ver criterios de exclusión 3.3.3), en ocasiones extradeportivos.

Esta línea de interpretación se puede justificar viendo que en estudios de hace 14-15 años (Woods et al., 2004), los hallazgos eran obtenidos con vuelta a competición en 18 días para lesiones de isquiosurales, pero, sin específicar si era como en este caso un grado 2 de lesión y en la unión miotendinosa proximal (ver apartado 3.3.1). 
Otro aspecto a valorar es que en 2004, las demandas del juego eran menos exigentes que en la actualidad, se refleja en estudios recientes donde se constatan números de

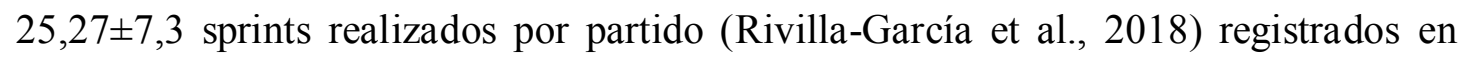

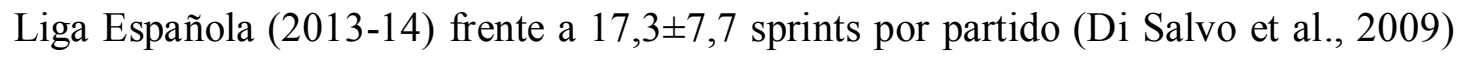
registrados en las temporadas 2002-04, o se sabe igualmente al valorar las importantes distancias recorridas superiores a $21 \mathrm{~km} / \mathrm{h}$ en diferentes trabajos con futbolistas profesionales siendo éstas entre 160-615m por competición (Taylor et al., 2017), y que no se pueden comparar con décadas atrás por no disponer de datos de GPS, pero si se estima en distancias suficientes para valorar la importante exigencia actual a este nivel.

Por ello, en este análisis, viendo que el dato de días de vuelta a competición, eran inferiores en 2002-2004, supuestamente con menos medios, los hallazgos de esta tesis doctoral podrían no parecer interesantes a este nivel, dando lugar a la interpretación de que ello no mejora lo de hace varias temporadas atrás.

En realidad, se considera que el registro "días de vuelta a competir", es una información que se debiese tomar con relatividad, puesto depende del calendario de las competiciones, y de otras decisiones adoptadas especialmente la del entrenador, así como de la comparativa en igualdad en cuanto a la severidad y localización exacta de la lesión, como publican trabajos recientes (Valle et al., 2018).

En este orden se cree que es interesante conocer cómo otros estudios más actuales registraron $24 \pm 13$ días en vuelta a competición para lesiones grado 2 del bíceps femoral (Hallén \& Ekstrand, 2014b), ó de 20,3 días de baja en roturas musculares y 18,3 días de media como baja para lesiones del bíceps femoral (Noya \& Sillero, 2011). Entendiendo ello, se considera lo importante que es evaluar el dato "momento vuelta al grupo" ya que no depende de la fecha de siguiente competición, sino de la 
evolución propia del lesionado y de la superación real de cargas, tareas y patrones de movimiento para volver a practicar la modalidad del fútbol.

Por ello, se puede constatar que el resultado expuesto de $16,94 \pm 1,56$ días, en cuanto a la vuelta al grupo, si es una fecha a tener en cuenta, ya que el deportista ha superado exigencias físico-deportivas para regresar a la incertidumbre del juego y a las habilidades abiertas (en entrenamiento con juegos reducidos y competitivos) (Bosch \& Ijzerman, 2016; Taberner, Allen, \& Cohen, 2019) una vez superada la lesión (modelo readaptación, ver apartado 3.5.2).

Previamente, se había mostrado en otros estudios con muestra de estudiantes (Kilcoyne et al., 2011) que los días de baja en lesiones grado 1-2 de isquiosurales podría estar en una media de 11,9 días y entre un rango de 5 a 23 días.

En definitva, se considera que puede ser altamente interesante el registro de la vuelta normalizada al grupo (Ardern et al., 2016; Buckthorpe et al., 2018) para obtener criterios y afrontar tareas colectivas, con condicionantes del propio juego, y a la vez, asimilarse a algoritmos vistos en revisiones de la literatura (Erickson \& Sherry, 2017b), contando además que emocionalmente es el momento en el que el deportista comienza a "verse recuperado" puesto puede entrenar y completar contenidos al mismo nivel que el resto del colectivo.

Del mismo modo, otros autores mostraron días de vuelta a competición por grupos musculares de los isquiosurales y por grados de lesión (Hallén \& Ekstrand, 2014b). 


\begin{tabular}{|c|c|c|c|c|c|}
\hline & N & Time to RTP (days) & Reinjury $\leq 2$ months & Reinjury $\leq 6$ months & Reinjury $\leq 12$ months \\
\hline Overall & 70 & $24.5 \pm 8.9$ & $6(8.6 \%)$ & $8(11.4 \%)$ & $11(15.7 \%)$ \\
\hline No intramuscular tendon disruption & 41 & $22.2 \pm 7.4$ & $3(7.3 \%)$ & $4(9.8 \%)$ & $6(14.6 \%)$ \\
\hline Intramuscular tendon disruption & 29 & $27.7 \pm 10.0^{*}$ & $3(10.3 \%)$ & $4(13.8 \%)$ & $5(17.2 \%)$ \\
\hline$<50 \%$ of tendon CSA & 5 & $24.0 \pm 9.7^{*}$ & $0(0 \%)$ & $0(0 \%)$ & $0(0 \%)$ \\
\hline $50 \%-99 \%$ of tendon CSA & 12 & $25.3 \pm 8.6^{*}$ & $1(8.3 \%)$ & $2(16.7 \%)$ & $3(25.0 \%)$ \\
\hline $100 \%$ of tendon CSA & 12 & $31.6 \pm 10.9 \dagger$ & $2(16.7 \%)$ & $2(16.7 \%)$ & $2(16.7 \%)$ \\
\hline No waviness & 53 & $22.6 \pm 7.5$ & $3(5.7 \%)$ & $5(9.4 \%)$ & $8(15.1 \%)$ \\
\hline Waviness & 17 & $30.2 \pm 10.8 \neq$ & $3(17.6 \%)$ & $3(17.6 \%)$ & $3(17.6 \%)$ \\
\hline
\end{tabular}

Figura 41. Días de vuelta a competición tras lesión (Van der Made et al., 2017)

\subsection{Discusión sobre resultados PRE-REA-GRUPO}

Al comparar cómo se desarrolló el programa de readaptación (intervención del estudio) con datos de sujetos sanos y en competición (PRE), se evalúa la progresión de las cargas en dicho proceso de readaptación y a su vez, los efectos del desentrenamiento que pudo producir esa lesión (figura 35).

Se observa cómo en seis variables como son "explosive distance", $\mathrm{n}^{\mathrm{o}}$ de impactos >7,1 g,Max_Acc y Max_speed, Avg_speed y V3 (figura 35) los valores están aún muy a favor del momento PRE, es decir, es posible interpretar que no fueron mejorados por la intervención, una vez completados los 13 ítems del programa.

Es sugerible interprear que variables del perfil explosivo y choques y/o cantidad de cambios de dirección con oposición, estaban siendo acondicionadas en el proceso de readaptación (Dos'Santos et al., 2018), y aún presentaban mejores valores en el estado Pre-lesión. 
Se verificó que en este momento del proceso, en la readaptación aún era pronto para juzgar qúe estaba ocurriendo si solamente se contase con la información de estas variables.

Únicamente con esta información de lo ocurrido en la intervención respecto al estado pre-lesión (figura 35), no se podría ser concluyente, aunque en relación a estas variables explosivas en otros procesos de readaptación, ya se conocía previamente evoluciones similares (Ehrmann et al., 2016; Suárez-Arrones et al., 2015a).

Por tanto, esta interpretación se completó viendo lo ocurrido en las comparaciones entre PRE-GRUPO (figura 36) e incluso viendo lo ocurrido más adelante en las comparaciones entre competiciones PRE-C1 (figura 38), una vez superada la lesión.

Continuando con el análisis de la comparación PRE-REA (figura 35), se observó que hay otras variables con resultados reseñables como son el "work to rest ratio", y el no de sprints/min, donde a pesar de estar en fase de readaptación, presentaron valores similares en la readaptación. Todo ello se podría interpretar entendiendo lo comparado en ese momento (jugador readaptándose de lesión “versus" estado PRE) y lógicamente también a través de los contenidos del propio programa de readaptación ya que en los ítems 3,5 y 10 hay tareas analíticas de carrera donde los metros recorridos en carrera $(>7 \mathrm{~km} / \mathrm{h})$ son mayores que los recorridos andando $(<7 \mathrm{~km} / \mathrm{h})$.

Sin embargo, en la propia competición (momento PRE) hay momentos de marcha que suponen hasta el 30\% del total de los metros recorridos, especialmente cuándo no interviene en acciones específicas (Gaudino et al., 2014; Suárez-Arrones et al., 2015a), y que justifican que lo importante no es correr mucho, sino el perfil de los esfuerzos junto con otros factores para ser determinante en el juego (Mallo et al., 2015; RivillaGarcía et al., 2018). 
En este momento del proceso (comparación PRE-REA), como es lógico, ninguna de las 13 variables analizadas, superó registros REA respecto a valores PRE, sin embargo fué llamativo, que estas 2 variables citadas $\left(\mathrm{n}^{\mathrm{o}}\right.$ sprints $>21 \mathrm{~km} / \mathrm{h} / \mathrm{min}$ y WTRR ), estuvieron en zona de diferencias triviales ("unclear"), es decir, no perdieron valores importantes en cuanto a registros respecto a PRE, aspecto que sirvió para justificar el interés de los ítems del modelo, consiguiendo en estas variables acercarse a la realidad competitiva, objetivo determinante en la readaptación de la lesión en la unión miotendinosa proximal del bíceps femoral.

Es cierto, que siendo conocedor de la evolución posterior (comparación PREGRUPO) (figura 36), especialmente de la variable $\mathrm{n}^{\mathrm{o}}$ sprints $>21 \mathrm{~km} / \mathrm{h} / \mathrm{min}$, en donde aunque, no llegaban a apreciarse mejoras significativas en GRUPO, respecto a PRE, se vió que el sprint es una variable muy recuperada en momentos de entrenar con el grupo, respecto a estado PRE. Es decir, haber pasado por el modelo de entrenamiento (REA) y ser evaluado en fase GRUPO, implicó a nivel de número de sprints/min que los isquiosurales soportaban un similar $n^{o}$ de acciones de alta intensidad por minuto de solicitación similar a la situación pre-lesión. De igual forma el número de aceleraciones y desaceleraciones/min a ambos perfiles de intensidad (Acc1-Acc2) especialmente las de baja intensidad (2,9-4 m/s2), fueron también muy similares en el momento GRUPO respecto a PRE (figura 36), aspecto que justifica la intervención dado el perfil de tareas propuesto en ítems 8,9,10.

Posteriormente se vió que las generadas por el programa son adaptaciones suficientes, es decir, los participantes no se desentrenaron para sprintar, esto a su vez puede justificar, lo ocurrido en fases posteriores (C1 respecto a PRE en figura 38) o incluso aún más posteriores (C2 respecto a PRE en figura 39). 
En trabajos previos, aunque en futbolistas amateurs (Casamichana \& Castellano, 2011) se vislumbraba la sospecha de que las demandas físico-deportivas en entrenamiento distaban de las exigidas y realizadas en competición, aunque en esta tesis doctoral se comparó entrenamiento en la fase de readaptación con la situación pre-lesión (competición), con las peculiaridades especiales que esas situaciones tienen por tratarse de un proceso de lesión (Caparrós et al., 2017).

Al continuar evaluando cómo se comportaron las variables en el proceso donde los deportistas estaban readaptándose, y en donde se comparó el momento de entrenamiento individual (REA) con valores previos en estados de no lesión (PRE), se pudó justificar la progresión en la consecución del aspecto condicional y funcional con el hecho de que los participantes no sufrieron recidivas, aspecto que se mostró como hecho frecuente (Malliaropoulos et al., 2010; Orchard \& Seward, 2002).

Desde el punto de vista de los efectos del desentrenamiento,se vió que no fué tal (figura 35) y con ello, con la implementación del programa durante la readaptación, se evitó lo que se cita en la literatura durante procesos de lesión muscular donde algunos trabajos muestran pérdidas a nivel muscular, cardiovascular, metabólicas y demás (Kadi et al., 2004; Klausen, Andersen, \& Pelle, 1981; Mujika, 2000).

Respecto a valorar lo ocurrido entre momento grupo respecto a cómo estaban deportistas antes de la lesión, se ve en la figura 36, cómo prácticamente hay 12 variables (especialmente Avg_speed) que no alcanzan valores similares durante el momento grupo (excepto $\mathrm{n}^{\mathrm{o}}$ de sprints/min), pero se entiende con la información posterior en el proceso (comparación PRE-C1 en figura 38) que las variables están en proceso de consecución de valores necesarios para devolver a la competición al deportista, y que en ese momento GRUPO, a pesar de tener criterios basados en la 
superación de tareas del modelo, las variables de rendimiento no son mejores que en estado PRE (figura 36).

Otros estudios recientes (Duhig et al., 2016) registran parámetros de rendimiento, como distancia total, metros recorridos $>24 \mathrm{~km} / \mathrm{h}$ y aceleraciones y desaceleraciones por encima de $3 \mathrm{~m} / \mathrm{s} 2$ con GPS en las 4 semanas previas a producirse lesiones de isquiosurales en fútbol Australiano, y posteriormente registran parámetros de mayor riesgo en cuanto a volumen recorrido a alta intensidad $(>24 \mathrm{~km} / \mathrm{h})$ en la semana previa a la lesión.

En estas situaciones se conoce cómo otros autores y equipos de investigación también registraron datos previos para después usarlos como referencia en momento grupo y estudiaron variables relacionadas con desaceleraciones y cambios de dirección (Dos'Santos et al., 2018).

Evaluando el proceso de readaptación, y usando la comparación REA-GRUPO (figura 36), es posible reseñar lo ocurido con variables como explosive distance / min, $\mathrm{n}^{\mathrm{o}} \mathrm{de}$ impacts $>7.1$ g, y V3, Max_speed, Max_Acc (figura 42) donde se concluyó que los deportistas pasando por la intervención, consiguieron mejorar estas variables en la vuelta al grupo, justificando con ello de igual forma, la validez y eficacia del modelo. 

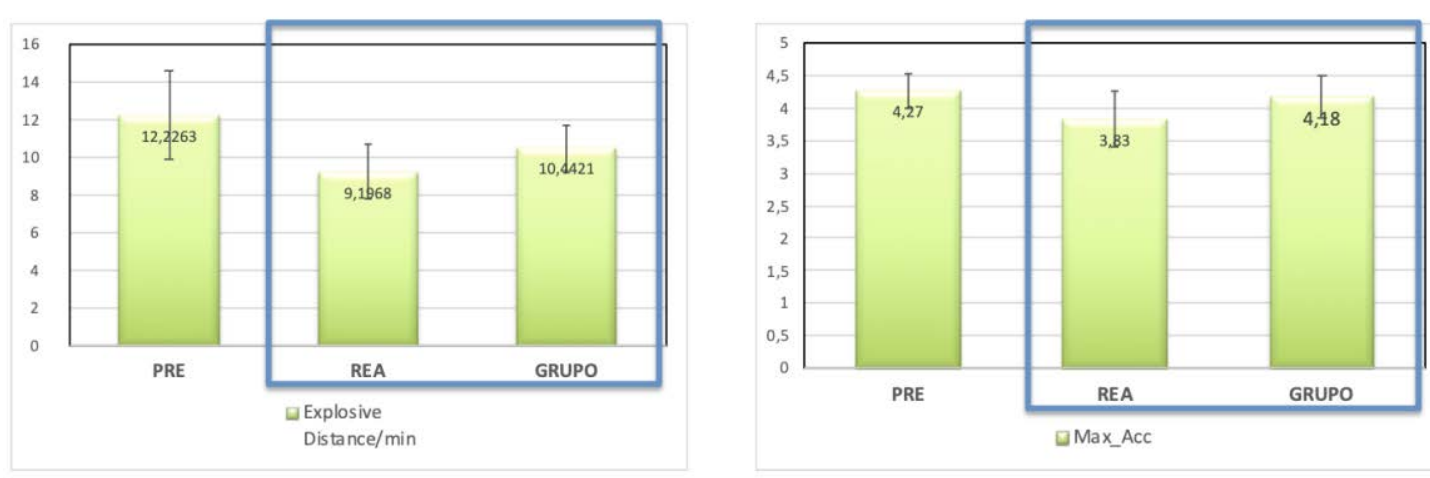

Figura 42. Valores obtenidos en explosive distance (m/min) y en Max_Acc (m/s2) en momentos REA-GRUPO

Se estimó, que otras variables como son $n^{o}$ sprints/min o $n^{o}$ de RSA/min, V2 (figura 36) estuvieron bastante igualadas ya en situación de vuelta al grupo, e incluso cobraron mejores valores en fase de readaptación, en parte justificado por ítems como el 9 o el 10, donde se entrenaron tareas explosivas, incluso tareas con 2 series de repetición de sprints “double repeated sprints ability”, y por tanto se observó que en ese perfil de entrenamiento de readaptación, se provocaron suficientes e idóneas adaptaciones que se comportaron como protectoras de la estructura lesionada (UMT PROX BF). Además. a nivel condicional, ello supuso óptimos estímulos para obtener mejoras en variables como Max_speed, $\mathrm{n}^{\circ}$ de impactos $>7,1 \mathrm{~g}$, Acc1-Acc2, explosive distance etc. (ver figura 36).

Se interpretó, que posteriormente en tareas colectivas no es necesario que ciertos parámetros sean mejores, para que el proceso fuese validado, como es el caso de Avg_speed, o incluso V1,V2 ó V3, donde se observaron valores no igualados durante la readaptación, pero a la vez suficientes entendiendo lo ocurrido a posteriori al comparar la llegada al grupo de los participantes respecto a su situación pre-lesión (figura 35). 
Por tanto, el análisis de la comparación REA-GRUPO (figura 36) sugirió una gran justificación de la intervención, y una confirmación de lo aceptado por el panel de expertos (Tabla 7). Se considera ésta una de las grandes justificaciones del programa de entrenamiento de la intervención.

Los participantes consiguieron importantes mejoras en la fase GRUPO, momento donde los deportistas especialmente en los juegos reducidos, obtuvieron mejores resultados en las variables medidas respecto a REA, debido a la especificidad de los propios contenidos del modelo.

Todo ello lo sustenta lo entrenado en la intervención, con mejoras importantes en variables explosivas (máxima aceleración, explosive distance..) y en donde tareas de juegos reducidos, el deportista presenta mejores parámetros debido a la especificidad en la readaptación (intervención).

Reseñable también, lo ocurrido con 2 variables, como son WTRR y Avg_speed, donde aún son mejores en el momento REA (intervención), posiblemente debido a trabajos analíticos desarrollados en los ítems 3,5 y 10 del modelo, lo que igualmente sirve para refrendar lo comentado anteriormente (figura 43)

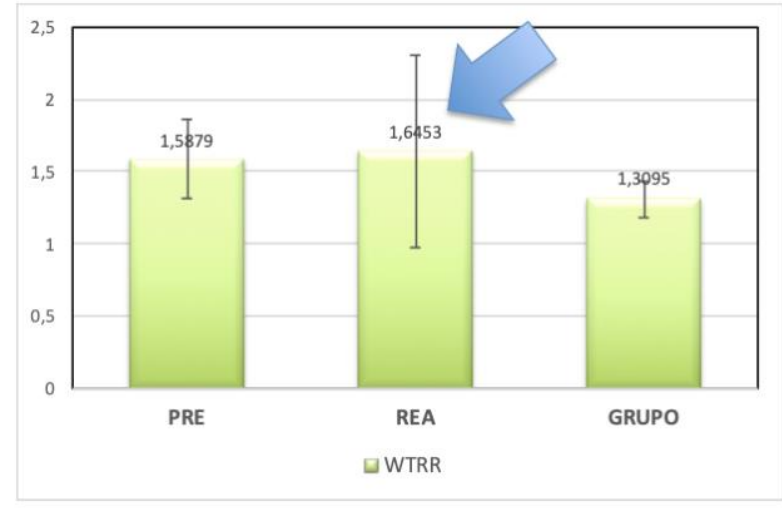

Figura 43. Valores obtenidos en "work to rest ratio" en momentos PRE-REAGRUPO 
Otro aspecto fundamental en esta discusión, es que es posible justificar cómo se puede trabajar la prevención de lesiones de isquiosurales a través de entrenamiento a altas velocidades (Edouard et al., 2018; Gabbett, 2017).

Algunos autores ya publicaron recientemente aspectos relacionados con tareas de RSA y agilidad en contenidos de readaptación, aunque fuera estudio de casos (Mendiguchia et al., 2016) o en otro perfil de estudios (Manouras, Papanikolaou, Karatrantou, Kouvarakis, \& Gerodimos, 2016) donde se vió que ítems realizados en esta intervención a este nivel de entrenamiento a altas velocidades puede ser válida para obtener estas mejoras en momento grupo, y lógicamente, para ir después a la competición (Czuppon, Racette, Klein, \& Harris-Hayes, 2014; Tol et al., 2014).

Ya existen algunos modelos en la literatura, que hacen también referencia al entrenamiento de gestos y movimientos para readaptar patologías, por ejemplo en lesiones de zona inguinal ("groin pain”) en futbolistas profesionales (King et al., 2018) y apoyan propuestas de entrenamiento multidireccional y multiplanar, con tareas basadas en el cambio de dirección (COD), agilidad y explosividad a nivel unitaleral (King et al., 2018).

\subsection{Discusión sobre resultados PRE-C1-C2}

Para interpretar lo obtenido con los 19 sujetos de la muestra en relación a perfiles de esfuerzos no homogéneos entre si, en varias variables del GPS, se ha de entender que son dependientes de las demarcarciones en el juego (figuras 37-39).

Los resultados obtenidos en esta tesis doctoral son similares a los obtenidos en otros trabajos donde se evaluó con sistemas tracking como Mediacoach® por 
demarcaciones o puestos de juego (Rivilla-García et al., 2018) o con GPS según demarcaciones (Gregson, Drust, Atkinson, \& Salvo, 2010).

Resulta reseñable la gran varianza de datos obtenida en diversas variables, como $\mathrm{n}^{\circ}$ RSA/minuto o $\mathrm{n}^{\mathrm{o}}$ sprints/minuto. No obstante ello por supuesto no implica la obtención de hallazgos reseñables, por ejemplo en la comparación de los datos de velocidad 3 entre el momento PRE y C1 se observó una mejora pequeña de 31,40\% (IC 90\% 17,16-43,20\%) (ver Tabla 13) o en $\mathrm{n}^{\mathrm{o}} \mathrm{RSA} / \mathrm{min}$ igualmente comparando PRE-C1 una mejora pequeña de 21,65\% (IC 90\% (2,44-37,07\%) (ver Tabla 14).

Ello hace entender, lo ocurrido en relación al regreso a las competiciones de los participantes recuperados de esta lesión (tanto en $\mathrm{C} 1$ como en $\mathrm{C} 2$ ), y se interpretan los porcentajes de mejoras favorables en el post-lesión (figuras 37-38) de la siguiente forma:

Basándose en lo obtenido en distancias a las velocidades evaluadas (especialmente V3), se observan importantes mejoras en las situaciones postlesión (figuras 37-39), viendo por tanto cómo la cadena extensora del miembro inferior de los participantes es capaz de generar más metros por minuto en dichas competiciones post-lesión, respecto a pre-lesión (figura 44).

Ello da lugar a justificar que la intervención (readaptación de la lesión) es un protocolo preventivo y eficaz, puesto que conocemos por otros estudios (Bourne et al., 2016; Hagglund et al., 2006; Timmins, 2017) que la lesión previa es uno de los factores de mayor incidencia en las recidivas, sin embargo, en este estudio, vemos que no ocurre así, y que la intervención durante el proceso lesivo, ha generado adaptaciones eficaces para obtener los resultados comentados, y que además no ha generado recidivas en 8 meses post-competición (Jiménez-Rubio et al., 2018). 


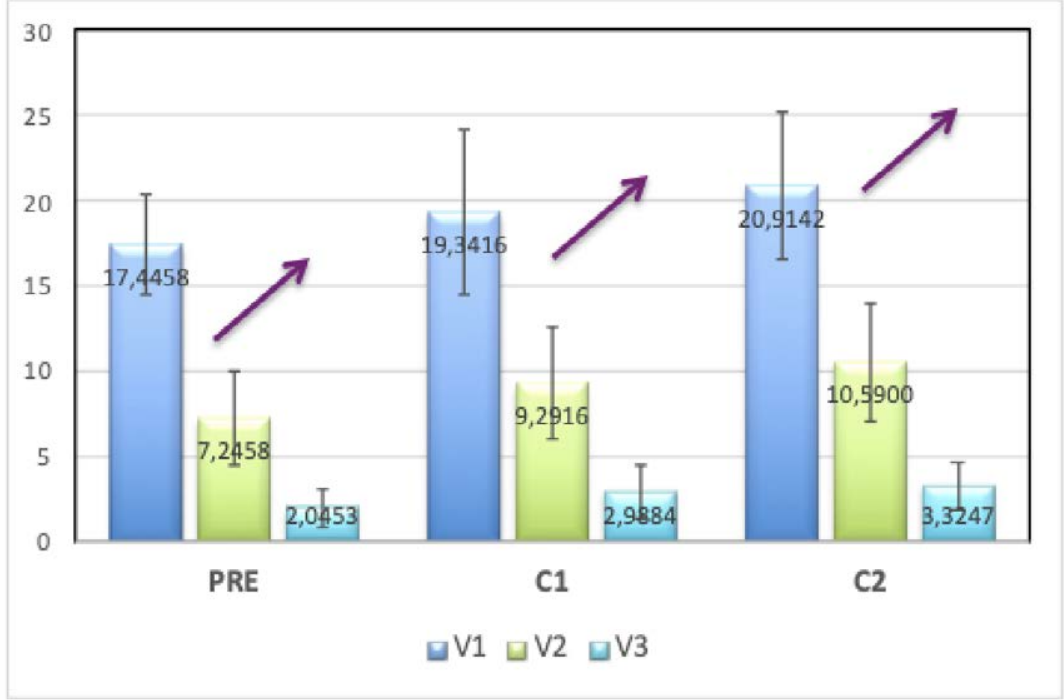

Figura 44. Valores de V1,V2 y V3 (m/min) en momentos PRE-C1-C2

En la comparación PRE-C1 (figura 38), igualmente se observó un importante \% de mejoras en variables máxima velocidad, máxima aceleración, impactos $>7,1 \mathrm{~g}$, “explosive distance" (m/min), $\mathrm{n}^{\mathrm{o}}$ de $\mathrm{RSA} / \mathrm{min}$, que llevó a interpretar lo importante que fué entrenar durante la lesión las fuerzas del vector horizontal (Mendiguchia et al., 2014; Morin et al., 2015; Schuermans, Danneels, et al., 2017), así como tareas con empujes y patrones de aceleración/desaceleración (ítems 8,9,10 del programa).

Estos resultados mostrados en esa figura 38, sugieren la interpretación de que el complejo muscular de los isquiosurales, no sólo se recuperó estructuralmente (Gabriel et al., 2017) y que se confirmó radiológicamente, sino que también funcionó mejor y pudo soportar una mayor carga competitiva alrededor de 6-10 semanas después de recuperarse para haber ido a la competición (figura 39).

Se constató una interesante recuperación fisiológica y funcional basada en óptimas velocidades de movimiento llevadas a cabo en los propios patrones de movimiento para volver a la fase de rendimiento (figuras 37-38-39). Estudios previos (Sole, Milosavljevic, Nicholson, \& Sullivan, 2012) mostraron que tras la lesión de 
isquiosurales había retardo en la activación respecto al miembro no lesionado, aspecto que hace cobrar un valor añadido a los resultados obtenidos tras la implementación de este programa de entrenamiento en fase de readaptación.

Esta tesis doctoral, es uno de los primeros estudios en el fútbol profesional que muestra una mejora en los parámetros de rendimiento de forma objetiva, durante la competición (figura 38), de jugadores que estaban previamente lesionados, y también una mejora durante las 6-10 semanas posteriores al regreso a la competición (figura 39).

Se considera que este hallazgo, es realmente interesante en un ámbito profesional donde en ocasiones se utilizan solamente criterios de imagen / radiológicos (Van der Horst et al., 2017) para tomar decisiones en cuanto a la vuelta al grupo, incluso para volver a la competición post-lesión desencadenando esas situaciones en una posible recidiva al no haber completado una correcta fase de readaptación durante el proceso.

Las investigaciones han demostrado que en el sprint a medida que la velocidad aumenta por encima de $\operatorname{los} 25 \mathrm{~km} / \mathrm{h}$, es la frecuencia de zancada la que aumenta y hay poco aumento en la longitud de zancada (Brigaud, 2016; Dorn, Schache, \& Pandy, 2012).

La explicación se cree que se debe a que el jugador desarrolla empujes sobre el suelo ("ground reaction forces") de forma más eficaz y frecuente conforme aumenta velocidad (Colyer, Nagahara, \& Salo, 2018; Tominaga, Ishii, Ueda, \& Kurokawa, 2016) resultando ello en un aumento de las fuerzas de reacción terrestres en el plano sagital (Tominaga et al., 2016) y generando a su vez sistemas preventivos y protectores sobre los isquiosurales (Duhig et al., 2016; Sweeting, Cormack, Morgan, \& Aughey, 2017). 
Esta pauta preventiva viene provocada al generar más tiempo de vuelo, debido a esos mencionados empujes sobre el suelo (Bosch \& Ijzerman, 2016). En esta intervención fueron desarrollándose eficazmente con el medio y antepié, y con el flexor del primer dedo del pié (Brigaud, 2016), lo que implica empujes desde distal a proximal disminuyendo las posibilidades de incorrecta mecánica en la transmisión de fuerzas.

Como consecuencia, se produce un aumento en las demandas energéticas y biomecánicas de musculatura del miembro inferior, de la zona central y de la musculatura estabilizadora de cadera (Raabe \& Chaudhari, 2018; Schache, Dorn, Williams, Brown, \& Pandy, 2014) especialmente los isquiosurales y los glúteos que son responsables de producir esas fuerzas horizontales y generar eficacia en el movimiento (Morin et al., 2015; Schuermans, Danneels, et al., 2017).

En esa situación, los isquiosurales trabajan en la extensión de la cadera junto al glúteo mayor, aspecto clave en la óptima eficiencia mecánica mientras realiza el gesto de triple extensión (tobillo-rodilla-cadera) de empuje contra el suelo (Alcaraz et al., 2018; Bosch \& Ijzerman, 2016; Schuermans, Van Tiggelen, et al., 2017).

El programa de readaptación realizado por los participantes incluía tareas de este perfil (ítem 8) a través de empujes con trineos y previamente en la situación anterior a la llegada al campo (a través de "sled push" - Tabla 5)

Claramente, ello indica que los isquiosurales y los glúteos de los participantes fueron capaces de soportar mas volumen de cargas post-lesión y tenían por tanto una mayor capacidad para recorrer más distancia a velocidades de sprint (>25,1 km.h) (V3) así como ser capaces de alcanzar mayores picos en velocidad máxima en las competiciones en donde regresaron al juego ( $\mathrm{C} 1$ y $\mathrm{C} 2)$ en comparación a su estado previo a la lesión (PRE) (figuras 37-38). 
Se considera ésta una de los principales fortalezas del modelo utilizado en la readaptación y en la fase de trabajo previa al campo (momento "indoor") con tareas entrenadas de este perfil en cadena cinética cerrada (CCC) en los planos frontal y sagital (Tabla 5) (Tyler, Schmitt, Nicholas, \& McHugh, 2017).

Si a ello se le suma lo esgrimido en publicaciones muy recientes (Bramah, Preece, Gill, \& Herrington, 2018) donde se analizan las posibles disfunciones en la carrera como causa de lesiones en deportistas sprinters, la eficacia de la intervención se cree que es tangible y se muestra en las figuras 37-39.

Otra observación importante con respecto a estas variables fué que al comparar los datos anteriores a la lesión (PRE) con los datos posteriores a la recuperación registrados de seis a diez semanas después del regreso al juego (Figura 39), los cambios favorables postlesión concretamente en las variables Max Speed, Max_Acc y en $n^{\circ}$ de $\mathrm{RSA} / \mathrm{min}$, al igual que las 3 distancias evaluadas a 3 velocidades diferentes V1, V2 y V3 (figura 44).

La obtención de estos resultados justificaron el rechazo de la hipótesis de esta tesis doctoral (ver apartado 6).

Esto resalta la importancia de continuar entrenando tareas explosivas post-lesión relacionadas con el sprint (Sweeting et al., 2017) y de entrenar de forma explosiva y a alta intensidad como mecnismo protector- preventivo de los isquiosurales, aunque parezca una paradoja (Gabbett, 2017; Gabbett \& Whiteley, 2017).

Como se puede verificar, en registros actuales, es conocido cómo en un partido de

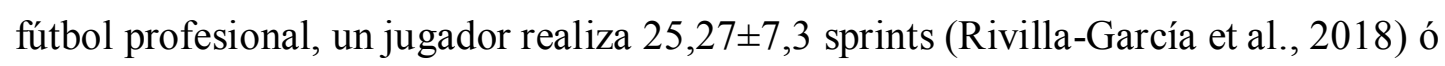
entre 7-61 sprints en revisiones con diferentes disciplinas (Taylor et al., 2017) ó 
26,5 \pm 12.6 sprints (Gregson et al., 2010) en liga de fútbol profesional inglesa registrados durante las temporadas 2004-2006.

A partir de ahí, se entiende que cuanto mejor se entrenan los patrones de aceleración y las mecánicas del sprint, no sólo se disminuye el riesgo de volver a lesionarse, ya que el jugador puede tolerar las cargas biomecánicas que estas velocidades implican, sino que también hay una mejora constante en estos parámetros de rendimiento del sprint (Gabbett, 2016; Malone et al., 2017), por ejemplo visto en mejoras de variable RSA/min en C2 respecto a PRE (figura 39) con una mejora (moderada) del 32,24\% (ver Tabla 14).

Por todo ello, esto no sólo demuestra cómo la intervención preparó a los jugadores para mejorar constantemente el rendimiento, sino también demuestra que fué efectivo en la prevención de lesiones.

Resaltar que ninguno de los jugadores que participaron en el programa sufrió una nueva lesión (recidiva) después de participar en este programa durante los 8 siguientes meses al regreso a la competición (Jiménez-Rubio et al., 2018).

Como se muestra en el apartado 3.5.2, el programa de readaptación se centró en el desarrollo de la fuerza del tren inferior e incluyó ejercicios de control neuromuscular, corrección de mecánicas del movimiento así como tareas a alta velocidad y RSA, mecánicas condicionales a través de los cambios de dirección y empujes sobre el suelo junto con bloques de trabajo táctico específico del futbolista lesionado (ítems $1,6,8,9,10,11,12)$

Finalmente se ejecutaron acciones con similitud de mecanismos lesionales con objeto de proteger la estructura lesionada (ítem 13). 
Se constató tras esta implementación (figuras 37-38) una mejor tolerancia a cargas de trabajo y un menor riesgo de lesiones en los deportistas (Duhig et al., 2016; Gabbett, 2016). Todo este proceso permitió disponer de criterios objetivos para determinar el retorno al juego de deportistas lesionados en isquiosurales, hallazgos que complementan a los de estudios recientes (Zambaldi et al., 2017).

Otros trabajos mostraron que los isquiosurales, después de una lesión, primero necesitan generar fuerzas a velocidades más bajas controladas durante un período mantenido de tiempo (mejorar perfil fuerza-tiempo) (Gabbett \& Whiteley, 2017) y sólo entonces pueden generar las fuerzas explosivas requeridas para soportar velocidades de sprint, de ahí la importancia del bloque isométrico a una sóla pierna en cadena cinética cerrada (Eklund et al., 2014; Van Hooren \& Bosch, 2017). De ese mismo modo se orientó este programa.

La participación regular del jugador en los partidos y en los entrenamientos, le permitió obtener mejoras en las distancias recorridas a altas intensidades (14,4-19,7 $\mathrm{km} / \mathrm{h})(=\mathrm{V} 1)$ y en la velocidad media (Avg_speed) de seis a diez semanas después de la vuelta al juego (figura 39) y durante la vuelta al juego (figura 38), en comparación con los datos anteriores a la lesión.

La ausencia de un jugador de un entrenamiento regular no sólo afecta al músculo lesionado, sino que también afecta a las estructuras circundantes, lo que podría tener un efecto adverso en el rendimiento del jugador en el campo. Esto podría deberse a que el sistema nervioso central funciona de manera diferente después de una lesión, especialmente si se subestima la reeducación (Mueller, 2007) y solamente se enfoca la recuperación de forma analítica en la estructura lesionada.

Las mejoras del jugador en la variable máxima aceleración, muestra una vez más, que el programa de intervención no sólo se centró en la función muscular lesionada, sino 
también en todas las estructuras de la parte inferior del cuerpo (tibial posterior, musculatura intrínseca del pié, etc), incluyendo el mencionado funcionamiento del sistema nervioso central, lo que permitió que el jugador volviera a jugar y, posteriormente, el estado físico adquirido en las siguientes competiciones permitió al jugador mejorar estos parámetros (figura 39).

Además, parecía haber una gran variación en los datos relacionados con variables como $\mathrm{n}^{\mathrm{o}}$ de RSA/min o $\mathrm{n}^{\mathrm{o}}$ sprints/min (figura 46). Esto puede explicarse por el hecho de que el estudio no separó a los jugadores por posición y los datos del partido se recogieron cuándo el jugador completó un mínimo de 45 minutos de juego.

A través de mediacoach $®$ (sitema tracking), un estudio reciente mostró cómo según la posición del jugador y el perfil de esfuerzos realizados estaba relacionado con la especificidad de los parámetros de rendimiento físico en la temporada de la Liga española (Rivilla-García et al., 2018), misma competición en la que participaron los jugadores que da lugar a esta tesis doctoral.

Lo más reseñable desde el punto de vista de las mejoras entre los valores PRE y los valores de mantenimiento en la competición (C2) es que las variables aceleración máxima y velocidad máxima registraron mejoras muy reseñables en competición post-lesión (ver figura 39), habiendo los deportistas pasado por un proceso lesivo y un modelo de readaptación que entre otros aspectos trató de mejorar estos registros.

No obstante, en este perfil de comparaciones, se ha de valorar la importancia del entrenamiento posterior en situaciones tras volver a competición donde el deportista hace trabajos específicos para protegerse de recidivas, y a su vez, registra mejoras de este perfil.

Ello viene a demostrar lo interesante que es entrenar los empujes, recepciones, la absorción de fuerzas y el sprint como mecanismos protectores en lesiones de los 
isquiosurales, conclusión que ya mostraron trabajos previos (Alcaraz et al., 2018; Edouard et al., 2018; Styles, Matthews, \& Comfort, 2016).

Otra cosa importante a tener en cuenta al interpretar los datos de velocidad media, es que ésta representa el promedio de todas las acciones de aceleración realizadas por el jugador, no sólo a altas intensidades y sprints, sino tambén a bajas velocidades. Ahí se vió como en este parámeto, no se obtuvieron diferencias significativas en la vuelta a la competición respecto a pre-lesión.

Ello lleva a hacer una interpretación de que el promedio de acciones de aceleración realizadas puede que no sea un parámetro determinante para evaluar las mejoras en el momento de vuelta a la competición.

En otro orden, se cree que es necesario entender o interpretar el parámetro "work to rest ratio", (representa una relación entre la distancia recorrida por el jugador por encima de $7 \mathrm{~km} / \mathrm{h}$ y la distancia recorrida por debajo de $7 \mathrm{~km} / \mathrm{h}$ ) para evaluar la distancia recorrida en acciones exigentes de carrera respecto a momentos de pausa en el juego. En ese sentido, se sabe que un jugador normalmente realiza la mayoría de los esfuerzos en competición por debajo de los 14 km/h (Figura 26) (Bradley et al., 2009) y esta variable, WTRR, puede que no sea determinante para el rendimiento del jugador en competición, sin embargo se considera que es interesante registrarla para evaluar la capacidad que va adquiriendo el jugador en el proceso de readaptación (figura 43).

Asimismo, se considera importante destacar que de seis a diez semanas después de la vuelta al juego (C2), el jugador era capaz de generar mayores volúmenes a todas las intensidades por encima de los $7 \mathrm{~km} / \mathrm{h}$, (figuras 37-39) lo que parecía contribuir a una mejora en las variables relacionadas con las velocidades más altas en competiciones post-lesión (Harnish \& Sabo, 2016). 
Los resultados de este análisis también indican que el jugador no sólo tenía un mejor control después de volver a jugar, sino que también parecía tener menos miedo de volver a lesionarse (Chao-Jung, Adam, Steven y Terese, 2016).

Al igual que otros estudios que han demostrado que la incorporación de entrenamiento excéntrico mantenido en el tiempo, podría ayudar a reducir el riesgo de volver a lesionarse los isquiosurales (Schmitt, Tyler y McHugh, 2012), esta tesis doctoral ha demostrado cómo un programa de readaptación específico en fútbol de alto nivel no sólo previene las lesiones sino que también mejora los parámetros de altas velocidades y capacidades de repetir sprints, elementos claves en fútbol profesional, interpretando con ello, que la mejor prevención es la tolerancia a cargas altas generadas con el propio entrenamiento, especialmente a través de tareas con ciclo CEA (Tabla 6).

En esta línea, se mostró que en los deportes de equipo, las intervenciones posteriores a las lesiones de los músculos isquiosurales deben incluir la reeducación de los movimientos específicos del deporte para adaptarse a las necesidades y demandas del juego.

Un ejemplo de este tipo de ejercicio (CEA) es la inclusión de tareas con habilidades de sprint repetidas (Bishop, Girard, \& Méndez-Villanueva, 2011; Girard et al., 2011) y que están constatadas en el modelo implantado (Jiménez-Rubio et al., 2018).

Otra parte importante es la inclusión de tareas que trabajan en la mejora constante de las fuerzas de reacción del suelo para que los músculos isquiosurales y glúteos puedan soportar las mayores cargas biomecánicas que las altas velocidades implican en el deporte de élite. 
Un ejemplo de tal ejercicio son los empujes con elementos perfil "sled push", cuestas o trineos, tareas que han demostrado ser útiles para prevenir lesiones (Alcaraz et al., 2018; Martínez-Valencia et al., 2015).

En definitva, se puede afirmar que el programa de readaptación no sólo debe fortalecer la función del grupo muscular lesionado, sino también los aspectos coordinativos del movimiento y facilitar la reeducación de los movimientos teniendo en cuenta la actividad del sistema nervioso central. Esto permitiría al jugador volver al deporte con mejores parámetros de rendimiento físico y reducir el riesgo de volver a lesionarse.

Es cierto que, en esta línea, estudios previos han analizado la importancia de entrenar aceleraciones máximas y velocidades por encima de $21 \mathrm{~km} / \mathrm{h}$ en 1 os diferentes momentos de la zancada en el sprint (Chumanov, Schache, et al., 2012; Higashihara et al., 2018).

Sobre el sprint,la referencia más novedosa pasa por la publicación de Sweeting et al. (2017) donde definen qué es realmente un sprint. Previamente, otros autores (Dwyer \& Gabbett, 2012) ya hicieron referencia al hecho de que un sprint se defina como cualquier movimiento que alcanza o excede la velocidad umbral de sprint durante al menos 1 segundo y cualquier movimiento con una aceleración que se produzca dentro del $5 \%$ más alto de aceleraciones encontradas en el margen de velocidad correspondiente.

Lo más reseñable entre las comparaciones hechas entre las competiciones post-lesión, es decir, entre el C1 y C2 (figura 39), es a nivel de las variables explosive distance/min y Acc2, posiblemente derivado del propio entrenamiento explosivo y de carga metabólica a través de patrones de aceleración a alta intensidad $(>4 \mathrm{~m} / \mathrm{s} 2)$ 
(Buchheit, Al Haddad, et al., 2014) generados con tareas con sujetos ya habiendo superado la lesión y en constantes competiciones de alto nivel.

Cabe recordar que estos sujetos continuaron haciendo trabajo pliométrico y multidireccional tras el programa de entrenamiento "on field".

Reseñable también que en las 13 variables analizadas se observaron mejoras, a nivel de rendimiento físico en favor de $\mathrm{C} 2$, lo que permitió afirmar que el propio entrenamiento en campo, sumado al entrenamiento preventivo "indoor", junto con tareas de fuerza realizadas durante post-lesión, fueron elementos fundamentales de carga externa para obtener esos interesantes resultados, de clara interpretación protectora de la re-lesión (figura 39).

En la literatura existen escasos estudios que hagan seguimiento tras volver a competir en el regreso de una lesión (Fernández-Gonzalo et al., 2016; Van der Made et al., 2017) con lo que estos resultados obtenidos entre estas comparaciones podrían servir como referencia para que programas de fuerza y entrenamiento en campo postcompetición de este perfil, sirvan como justificación en esas etapas de los deportistas previamente lesionados.

Y para finalizar este apartado de discusión, comentar, que estudios de 2018 en relación a la rehabilitación de las lesiones, utilizaron igualmente el sistema de expertos para evaluar parámetros en deportistas en fase de recuperación de lesiones en cuanto a la fuerza, resistencia, agilidad y estabilidad, además, al igual que en esta investigación la V de aiken con validez > a 0,75 (Gómez-Piqueras et al., 2018). 
VI. Conclusiones 

En relación al primer objetivo general, se ha diseñado un programa de entrenamiento propuesto para jugadores de fútbol en momentos de lesión, y por tanto, se considera aplicable en la fase de readaptación y reentrenamiento para lesiones en la unión miotendinosa proximal del bíceps femoral.

En relación al segundo objetivo general, se ha confirmado la eficacia del programa propuesto ya que a través del mismo se consiguieron mejoras significativas en parámetros de rendimiento relevantes en el momento de vuelta a la competición, y mejoras significativas en el plazo de 6-10 semanas tras la vuelta a dicha competición.

En relación a los objetivos específicos, se ha validado un programa de entrenamiento a través de un panel de expertos para aplicarlo en el momento de lesión de la unión miotendinosa proximal del bíceps femoral en futbolistas.

$\checkmark$ Se ha conseguido determinar la utilidad del programa constatando la mejora de parámetros específicos de rendimiento del futbolista. Entre ellos, se ha confirmado una mejoría significativa en el momento post-lesión de la capacidad de hacer más metros/minuto a 3 velocidades diferentes, así como hacer mayor volumen de aceleraciones máximas, aumentar el pico de velocidad máxima $\mathrm{u}$ otros valores como el número de aceleraciones/desaceleraciones por minuto en dos perfiles de intensidad.

Se han establecido criterios para llevar al deportista a la fase de entrenamiento con el grupo (superar los ítems del programa de readaptación) y criterios para la vuelta a la competición, en lesiones de la unión miotendinosa proximal del bíceps femoral (poder acumular volumen de entrenamiento colectivo y superar juegos reducidos sin incidencias clínicas en la vuelta con el grupo post-lesión). 
$\checkmark$ Con estos resultados, se puede concluir, que el ejercicio (programa) aplicado con los principios del entrenamiento (progresión, individualización etc) es una herramienta muy válida para que una estructura lesionada sea funcional y capaz de soportar las demandas de la competición en fútbol de alto nivel.

\section{La hipótesis queda rechazada debido a que los valores post-lesión son significativamente mejores que los hallados en momento pre-lesión.}

\subsection{Perspectivas de investigación y futuras líneas de investigación}

La investigación desarrollada en esta tesis doctoral podría ser el inicio del desarrollo y propuesta de otros modelos de readaptación que tengan como objeto disminuir los riesgos de recidiva en otro perfil de lesiones incluso podría servir como base para mejorar en un futuro este mismo programa.

Sería interesante también investigar acerca de las valoraciones que se podrían realizar en la denominada fase "indoor" (ver Tabla 5) en donde a nivel de movilidad podrían obtenerse resultados y conclusiones interesantes a partir de las cuales avanzar en el proceso. Por ejemplo, la evaluación de la dorsiflexión de tobillo, la valoración de la movilidad de columna dorsal o la mayor o menor rotación interna/externa de la cadera así como la aparición o no de eversión en tobillo, normalmente por déficits del tibial posterior, situaciones que predisponen a mecánicas incorrectas y a procesos lesivos.

Todo ello debería realizarse para después ejecutar las tareas de forma aún más eficiente que el propio modelo propone, normalmente a una sola pierna, para optimizar las recepciones en el suelo y los empujes mencionados en el programa, que derivarán en los futuros cambios de dirección, muy necesarios en la modalidad deportiva del fútbol profesional. 
Respecto al futuro, consideramos que se necesitan más estudios sobre las variables específicas de rendimiento en fútbol en situaciones donde hay por medio una lesión deportiva, que ha generado una alteración en el entrenamiento y en la competición del deportista de alto nivel.

En este caso, se ha desarrollado una propuesta metodológica en una lesión concreta, la lesión del tercio proximal del bíceps femoral, pero hay una demanda importante de desarrollo de programas, criterios y progresiones en situaciones que se citan para lesiones en futbolistas, por ejemplo del recto anterior del cuádriceps, la lesión del adductor mediano y su relación con las pubalgias, así como las lesiones en el tríceps sural (gastrocnemio medial y sóleo), y que como se constata en la epidemiología de las lesiones publicada (Ekstrand, Hagglund, et al., 2011), son de altísima incidencia.

Dentro de las lesiones de los isquiosurales, una futura línea de investigación pasaría por tratar de elaborar metodologías de intervención en fases de readaptación basadas en si la lesión es a nivel proximal (caso de nuestra muestra) o a nivel distal, donde posiblemente, la aplicación de otros estímulos diferentes podrían ser válidos para obtener resultados concluyentes.

Por otra parte, en cuanto a la herramienta de medición, comentar que en la actualidad el mercado ofrece modernos sistemas tracking y el uso de Gps y Lps más modernos, que dan la posibilidad de valorar otros parámetros o variables que relacionan por ejermplo asimetrías en cuanto a apoyos y solicitaciones (p.ej "step balance”) en los empujes contra el suelo, o perfiles de aterrizajes en el contexto específico de un deporte de tanta incertidumbre como el fútbol profesional (jumps/min; avg/landings etc).

Quizá incluir en futuros estudios variables de estos perfiles podría ser interesante como predictores de problemas durante la readaptación o con jugadores sanos para 
disminuir riesgos, durante sus exposiciones en el entrenamiento y en el juego, incluso para identificar situaciones de riesgo en los deportistas.

Desde otro punto de vista, futuras líneas de investigación podrían enfocarse en evaluar los niveles de fuerza del miembro lesionado, ya que se desconoce el tiempo real que se tardan en recuperar (especialmente en situaciones post-lesión) estos niveles de fuerza explosiva. Por ello futuras líneas de investigación podrían también evaluar dichos niveles de fuerza durante el proceso de readaptación para tomar decisiones en cuanto al "return to train" y "return to play".

En consecuencia, estudios posteriores podrían estar enfocados en evaluar con diferentes herramientas el perfil de Fuerza -Velocidad, o la velocidad media propulsiva con la que los sujetos progresan durante la readaptación de sus lesiones.

Para finalizar este apartado, comentar que una interesante futura línea de investigación pasaría por poder desarrollar y validar otros modelos de readaptación para diferentes lesiones y que éstos se basasen en el análisis del movimiento (podría ser a través del video-análisis) en acciones como los aterrizajes, recepciones, impulsiones (empujes) y cambios de dirección que sirvieran como recurso en las propuestas de ejercicio en programas enfocados a la readaptación de lesiones articulares y musculares.

En otro orden de actuación, podrían elaborarse perfiles individuales en cuanto a esfuerzos y desde esa información hacer comparaciones por puestos de registros obtenidos por ejermplo en metros / minuto en diferentes velocidades. 


\subsection{Aplicaciones prácticas}

$\checkmark$ Tras lo concluido en esta tesis doctoral, se dispone de un programa de readaptación para fútbol, compuesto por 13 ítems que pueden utilizarse durante la recuperación de lesiones en musculatura isquiosural, concretamente en el tercio proximal.

$\checkmark$ Se obtuvieron criterios para devolver al deportista lesionado a las cargas que realiza el grupo, una vez superada su lesión.

$\checkmark$ Se conocieron cuantitativamente los resultados a nivel de las variables registradas en la vuelta a la competición y se pueden utilizar como referencia, para tomar decisiones con deportistas que se quieran llevar a la competición post-lesión, en este caso en una lesión concreta.

Esos registros, pueden ser útiles para analizar el progreso de la superación de tareas que se acerquen a las demandas competitivas, y podrían servir para interpretaciones en cuanto a esfuerzos y perfiles de movimientos que son capaces de realizar los lesionados, por ejemplo en cuanto a desaceleraciones / minuto de alta intensidad o "explosive distance/min" superadas para plantearse un entrenamiento colectivo o una vuelta a la competición reduciendo el riesgo a través de lo objetivo.

En otra línea, este trabajo permite utilizar tareas del perfil mostradas en el modelo, para evitar el desentrenamiento durante una lesión muscular. A través de las tareas “indoor” (Tabla 5) como con la progresión mostrada en el propio modelo (Tabla 6) ya que los resultados en el momento de vuelta al entrenamiento de grupo son especialmente mejores que en la fase de intervención, dando ello lugar a la interpretación de que las adaptaciones generadas con el entrenamiento durante la readaptación, evitan o reducen los 
conocidos efectos del desentrenamiento (Mujika, 2000; Mujika \& Padilla, 2001).

$\checkmark$ Se considera que otra gran aplicación es la de comprobar cómo se mantiene el deportista lesionado en la competición durante 6-10 semanas tras el regreso post-lesión.

$\checkmark$ Para finalizar, es interesante valorar que este programa de readaptación puede ser extrapolable a otros grupos de deportistas explosivos, haciendo las adaptaciones necesarias al lesionado en cuestión.

\subsection{Limitaciones}

Esta tesis doctoral fué realizada con una serie de limitaciones, que se fueron encontrando en cierta medida debido al perfil de la muestra y al contexto donde se realizaron las mediciones.

Dichas limitaciones fueron las siguientes:

$\checkmark$ No se disponía del registro de las variables en cada competición (actualmente ya se manejan esos registros dados los recursos materiales), y con ello,se hubiese podido haber utilizado siempre un PRE-test de 4 semanas, para los datos Pre-lesión.

Plantear este estudio con datos de 3-4 semanas previas a cada lesión es una futura línea de seguimiento de este estudio.

$\checkmark$ La literatura muestra estudios (Van der Made et al., 2017) donde se continuaron controlando jugadores recuperados de lesión hasta 12 meses después, sin embargo en esta tesis doctoral solo se controló lo ocurrido 6-10 semanas después de la vuelta a competición, aunque se constató que no se 
produjeron recidivas en 8 meses posteriores a la primera competición (Jiménez-Rubio et al., 2018).

$\checkmark$ No haber registrado durante mismo tiempo (aproximando a tiempo real de competición completa, sobre 90 minutos) en situaciones Pre-lesión.

Como justificación y explicación, cabe decir que en el ámbito profesional es casi imposible registrar 90 minutos en pretemporada, por circunstancias del juego y decisiones técnicas, que es donde se obtuvieron los datos PRE. Esa dificultad la demuestran estudios similares (Suárez-Arrones et al., 2015a).

Sólo se han evaluado y analizado los parámetros de entrenamiento (intervención del estudio $=$ REA) que realizan los sujetos durante la recuperación de estas lesiones, sin controlar con exactitud el aspecto terapéutico (progresión del tratamiento de fisioterapia), por no ser una disciplina de las Ciencias de la Actividad Física y del Deporte.

Aunque sí se confirma el uso de fisioterapia invasiva (Valera-Garrido et al., 2014) en el tratamiento de cada uno de los sujetos.

No se dispuso de datos con jugadores sanos (gupo control) durante el proceso PRE-C1-C2 para evaluar si hubo o no hubo cambios en los parámetros de rendimiento, respecto a los que participaron en el estudio.

Se pudo controlar e igualar la intervención (readaptación) pero no las fases del momento de volver al grupo, debido a los diferentes modelos de entrenamiento con jugadores sanos durante esa duración del estudio, con cambios de entrenador incluidos, donde los juegos reducidos "small sided games" se realizaron con muy diferentes parámetros en cuanto a intensidad, extensión del espacio, frecuencia y volumen de entrenamiento. 
$\checkmark$ No se evaluó ó registró con otra herramienta (p.ej encoder lineal o plataformas de fuerzas) los trabajos de fuerza explosiva propuestos y realizados con los deportistas en la fase de intervención, normalmente ejecutados en momentos previos (indoor) al desarrollo del entrenamiento en el medio específico del fútbol, el campo. Dicha limitación puede ser a la vez una futura línea de investigación. 
VII. Bibliografía 

Aguiar, A. C., Melbourne, T. I., \& Scrivner, C. W. (2009). Moment release rate of Cascadia tremor constrained by GPS. Journal of Geophysical Research, 114(B7), B00A05. doi:10.1029/2008JB005909

Aiken, L. R. (1980). Content Validity and Reliability of Single Items or Questionnaires. Educational and Psychological Measurement, 40(4), 955-959. doi:10.1177/001316448004000419

Aiken, L. R. (1985). Three Coefficients for Analyzing the Reliability and Validity of Ratings. Educational and Psychological Measurement, 45(1), 131-142. doi:10.1177/0013164485451012

Akenhead, R., French, D., Thompson, K. G., \& Hayes, P. R. (2014). The acceleration dependent validity and reliability of $10 \mathrm{~Hz}$ GPS. Journal of Science and Medicine in Sport, 17(5), 562-566. doi:10.1016/j.jsams.2013.08.005

Al Haddad, H., Méndez-Villanueva, A., Torreño, N., Munguia-Izquierdo, D., \& Suárez-Arrones, L. (2017). Variability of GPS-derived running performance during official matches in elite professional soccer players. The Journal of Sports Medicine and Physical Fitness, 58(10), 1439-1445. doi:10.23736/S00224707.17.07500-4

Alcaraz, P. E., Carlos-Vivas, J., Oponjuru, B. O., \& Martínez-Rodríguez, A. (2018). The Effectiveness of Resisted Sled Training (RST) for Sprint Performance: A Systematic Review and Meta-analysis. Sports Medicine, 1-23. doi:10.1007/s40279-018-0947-8

Alexiou, H., \& Coutts, A. J. (2008). A comparison of methods used for quantifying internal training load in women soccer players. International Journal of Sports Physiology and Performance, 3(3), 320-30.

Andrzejewski, M., Chmura, J., Pluta, B., \& Konarski, J. M. (2015). Sprinting 
Activities and Distance Covered by Top Level Europa League Soccer Players. International Journal of Sports Science \& Coaching, 10(1), 39-50. doi:10.1260/1747-9541.10.1.39

Anemaet, W. K., \& Hammerich, A. S. (2014). A Framework for Exercise Prescription. Topics in Geriatric Rehabilitation, 30(2), 79-101. doi:10.1097/TGR.0000000000000011

Ardern, C. L., Glasgow, P., Schneiders, A., Witvrouw, E., Clarsen, B., Cools, A., ... Bizzini, M. (2016). 2016 Consensus statement on return to sport from the First World Congress in Sports Physical. British Journal of Sports Medicine, 1-12. doi:10.1136/bjsports-2016-096278

Armfield, D. R., Kim, D. H.-M., Towers, J. D., Bradley, J. P., \& Robertson, D. D. (2006). Sports-Related Muscle Injury in the Lower Extremity. Clinics in Sports Medicine, 25(4), 803-842. doi:10.1016/j.csm.2006.06.011

Arnason, A., Andersen, T. E., Holme, I., Engebretsen, L., \& Bahr, R. (2008). Prevention of hamstring strains in elite soccer: An intervention study. Scandinavian Journal of Medicine and Science in Sports, 18(1), 40-48. doi:10.1111/j.1600-0838.2006.00634.x

Arnason, A., Sigurdsson, S. B., Gudmundsson, A., Holme, I., Engebretsen, L., \& Bahr, R. (2004). Risk Factors for Injuries in Football. The American Journal of Sports Medicine, 32(1_suppl), 5-16. doi:10.1177/0363546503258912

Askling, C., Karlsson, J., \& Thorstensson, A. (2003). Hamstring injury occurrence in elite soccer players after preseason strength training with eccentric overload. Scandinavian Journal of Medicine \& Science in Sports, 13(4), 244-250.

Askling, C., Malliaropoulos, N., \& Karlsson, J. (2012). High-speed running type or stretching-type of hamstring injuries makes a difference to treatment and 
prognosis. British Journal of Sports Medicine, 46(2), 86-87. doi:10.1136/bjsports-2011-090534

Askling, C., Nilsson, J., \& Thorstensson, A. (2010). A new hamstring test to complement the common clinical examination before return to sport after injury. Knee Surgery, Sports Traumatology, Arthroscopy, 18(12), 1798-1803. doi:10.1007/s00167-010-1265-3

Askling, C., Saartok, T., \& Thorstensson, A. (2006). Type of acute hamstring strain affects flexibility, strength, and time to return to pre-injury level. British Journal of Sports Medicine, 40(1), 40-44. doi:10.1136/bjsm.2005.018879

Askling, C., Tengvar, M., Saartok, T., \& Thorstensson, A. (2007). Acute first-time hamstring strains during slow-speed stretching: clinical, magnetic resonance imaging, and recovery characteristics. The American Journal of Sports Medicine, 35(10), 1716-1724. doi:10.1177/0363546507303563

Askling, C., Tengvar, M., Saartok, T., \& Thorstensson, A. (2008). Proximal hamstring strains of stretching type in different sports: injury situations, clinical and magnetic resonance imaging characteristics, and return to sport. The American Journal of Sports Medicine, 36(9), 1799-1804. doi: $10.1177 / 0363546508315892$

Askling, C., Tengvar, M., Tarassova, O., \& Thorstensson, A. (2014). Acute hamstring injuries in Swedish elite sprinters and jumpers: a prospective randomised controlled clinical trial comparing two rehabilitation protocols. British Journal of Sports Medicine, 48(7), 532-539. doi:10.1136/bjsports-2013-093214

Aughey, R. J. (2011). Applications of GPS technologies to field sports. International Journal of Sports Physiology and Performance, 6(3), 295-310. doi:10.1080/02640414.2013.868920 
Balague, N., Torrents, C., Hristovski, R., Davids, K., \& Araújo, D. (2013). Overview of complex systems in sport. Journal of Systems Science and Complexity, 26(1), 4-13. doi:10.1007/s11424-013-2285-0

Balius, R., Pedret, C., Blasi Brugué, M., Alomar, X., Rodas, G., J, P. A., \& Isabel, M. P. (2013). Clasificación de las lesiones musculares. In Lesiones musculares en el deporte (pp. 37-44). Madrid. Panamericana.

Balius, R., Pedret, C., \& Pujol, M. (2013). Lesión de los isquiosurales. In Lesiones musculares en el deporte (pp. 229-252). Madrid. Panamericana.

Barbero-Alvarez, J. C., Coutts, A., Granda, J., \& Castagna, C. (2010). The validity and reliability of a global positioning satellite system device to assess speed and repeated sprint ability (RSA) in athletes. Journal of Science and Medicine in Sport, 13(2), 232-5. doi:10.1016/j.jsams.2009.02.005

Barbero-Alvarez, J. C., López, M. G., Castagna, C., Romero, D. V., Blanchfield, A. W., \& Nakamura, F. Y. (2015). Game Demands of 7-a-Side Soccer in Young Players. Journal of Strength and Conditioning Research, 31(7); 1771-1779. doi:10.1519/JSC.0000000000001143

Barrett, S., Midgley, A., Reeves, M., Joel, T., Franklin, E., Heyworth, R., ... Lovell, R. (2016). The within-match patterns of locomotor efficiency during professional soccer match play: Implications for injury risk? Journal of Science and Medicine in Sport, 19(10), 810-815. doi:10.1016/J.JSAMS.2015.12.514

Barros, R. M. L., Misuta, M. S., Menezes, R. P., Figueroa, P. J., Moura, F. A., Cunha, S. A., ... Leite, N. J. (2007). Analysis of the distances covered by first division brazilian soccer players obtained with an automatic tracking method. Journal of Sports Science \& Medicine, 6(2), 233-42.

Bayer, M. L., Magnusson, S. P., Kjaer, M., \& Tendon Research Group Bispebjerg. 
(2017). Early versus Delayed Rehabilitation after Acute Muscle Injury. New England Journal of Medicine, 377(13), 1300-1301. doi:10.1056/NEJMc1708134 Beato, M., Bartolini, D., Ghia, G., \& Zamparo, P. (2016). Accuracy of a $10 \mathrm{~Hz}$ GPS Unit in Measuring Shuttle Velocity Performed at Different Speeds and Distances (5 - 20 M). Journal of Human Kinetics, 54, 15-22. doi:10.1515/hukin-2016-0031

Bengtsson, H., Ekstrand, J., \& Hägglund, M. (2013). Muscle injury rates in professional football increase with fixture congestion: an 11-year follow-up of the UEFA Champions League injury study. British Journal of Sports Medicine, 47(12), 743-747. doi:10.1136/bjsports-2013-092383

Bengtsson, H., Ekstrand, J., Walden, M., \& Hägglund, M. (2017). Muscle injury rate in professional football is higher in matches played within 5 days since the previous match: a 14-year prospective study with more than 130000 match observations. British Journal of Sports Medicine, 52; 1116-1122.

Bishop, D., Girard, O., \& Méndez-Villanueva, A. (2011). Repeated-sprint ability part II: Recommendations for training. Sports Medicine, 41(9), 741-756. doi:10.2165/11590560-000000000-00000

Blanch, P., \& Gabbett, T. (2016). Has the athlete trained enough to return to play safely? The acute:chronic workload ratio permits clinicians to quantify a player's risk of subsequent injury. British Journal of Sports Medicine, 50(8), 471-475. doi:10.1136/bjsports-2015-095445

Bloomfield, J., Polman, R., \& O’Donoghue, P. (2007). Physical Demands of Different Positions in FA Premier League Soccer. Journal of Sports Science \& Medicine, $6(1), 63-70$.

Borresen, J., \& Ian Lambert, M. (2009). The Quantification of Training Load, the Training Response and the Effect on Performance. Sports Medicine, 39(9), 779- 
795. doi:10.2165/11317780-000000000-00000

Bosch, F. (2016). Specificity within strength training. In Strength training and coordination: An Integrative Approach (pp. 181-226). Rotterdam (Netherlands). Uitgevers.

Bosch, F., \& Ijzerman, J. (2016). Running mechanics in injury prevention and performance. In J. D \& L. D (Eds.), Sport injury prevention and rehabilitation integrating medicine and science for performance solutions (pp. 106-120). New York (USA). Routledge.

Bourdon, P., \& Cardinale, M. (2017). Monitoring Athlete Training Loads: Consensus Statement. International Journal of Sports Physiology and Performance. 12 (S2) (161-170) doi:10.1123/IJSPP.2017-0208

Bourne, M. N., Timmins, R. G., Opar, D. A., Pizzari, T., Ruddy, J. D., Sims, C., ... Shield, A. J. (2017). An Evidence-Based Framework for Strengthening Exercises to Prevent Hamstring Injury. Sports Medicine. doi:10.1007/s40279-017-0796-x

Bourne, M. N., Williams, M., Opar, D. A., Al Najjar, A., Kerr, G. K., \& Shield, A. J. (2016). Impact of exercise selection on hamstring muscle activation. British Journal of Sports Medicine.

Bowen, L., Gross, A. S., Gimpel, M., Bruce-Low, S., \& Li, F.-X. (2019). Spikes in acute:chronic workload ratio (ACWR) associated with a 5-7 times greater injury rate in English Premier League football players: a comprehensive 3year study. British Journal of Sports Medicine, 0, 1-9 bjsports-2018-099422. doi:10.1136/bjsports-2018-099422

Bradley, P. S., \& Noakes, T. D. (2013). Match running performance fluctuations in elite soccer: Indicative of fatigue, pacing or situational influences? Journal of Sports Sciences, 31(15), 1627-1638. doi:10.1080/02640414.2013.796062 
Bradley, P. S., Olsen, P. D., \& Portas, M. D. (2007). The effect of static, ballistic, and proprioceptive neuromuscular facilitation stretching on vertical jump performance. Journal of Strength and Conditioning Research, 21(1), 223-6. doi:10.1519/R-21046.1

Bradley,P.S., Sheldon, W., Wooster, B., Olsen, P., Boanas, P., \& Krustup,P. (2009). High-intensity running in English FA Premier League soccer matches. Journal of Sports Sciences, 27 (2), 159-168. doi: 10.1080/02640410802512775

Bramah, C., Preece, S. J., Gill, N., \& Herrington, L. (2018). Is There a Pathological Gait Associated With Common Soft Tissue Running Injuries? The American Journal of Sports Medicine, 036354651879365. doi:10.1177/0363546518793657

Brigaud. (2016). Apoyo con el antepié: Beneficios, biomecánica y postura. In La carrera. Postura, biomecánica y rendimiento (1 ${ }^{\mathrm{a}}$, pp. 59-92). Badalona (España). Paidotribo.

Brukner, P., \& Connell, D. (2015). 'Serious thigh muscle strains': beware the intramuscular tendon which plays an important role in difficult hamstring and quadriceps muscle strains. British Journal of Sports Medicine, 50; 205-208.

Brukner, P., Nealon, A., Morgan, C., Burgess, D., \& Dunn, A. (2014). Recurrent hamstring muscle injury: Applying the limited evidence in the professional football setting with a seven-point programme. British Journal of Sports Medicine, 48(11) 929-938. doi:10.1136/bjsports-2012-091400

Buchheit, M., Al Haddad, H., Simpson, B. M., Palazzi, D., Bourdon, P. C., Di Salvo, V., \& Méndez-Villanueva, A. (2014). Monitoring accelerations with GPS in football: time to slow down? International Journal of Sports Physiology and Performance, 9(3), 442-445. doi:10.1123/ijspp.2013-0187

Buchheit, M., Allen, A., Poon, T. K., Modonutti, M., Gregson, W., \& Di Salvo, V. 
(2014). Integrating different tracking systems in football: multiple camera semiautomatic system, local position measurement and GPS technologies. Journal of Sports Sciences. doi:10.1080/02640414.2014.942687

Buchheit, M., Gray, A., \& Morin, J. B. (2015). Assessing stride variables and vertical stiffness with GPS-embedded accelerometers: Preliminary insights for the monitoring of neuromuscular fatigue on the field. Journal of Sports Science and Medicine, 14(4), 698-701.

Buchheit, M., Haddad, H. Al, Simpson, B. M., Palazzi, D., Bourdon, P. C., Salvo, V. Di, \& Méndez-Villanueva, A. (2014). Monitoring accelerations with gps in football: Time to slow down. International Journal of Sports Physiology and Performance, 9(3), 442-445. doi:10.1123/IJSPP.2013-0187

Buchheit, M., Méndez-Villanueva, A., Delhomel, G., Brughelli, M., \& Ahmaidi, S. (2010). Improving Repeated Sprint Ability in Young Elite Soccer Players: Repeated Shuttle Sprints Vs. Explosive Strength Training. Journal of Strength and Conditioning 24(10), Research, 2715-2722. doi:10.1519/JSC.0b013e3181bf0223

Buckthorpe, M., Frizziero, A., \& Roi, G. S. (2018). Update on functional recovery process for the injured athlete: return to sport continuum redefined. British Journal of Sports Medicine, bjsports-2018-099341. doi:10.1136/bjsports-2018099341

Buckthorpe, M., Gimpel, M., Wright, S., Sturdy, T., \& Stride, M. (2017). Hamstring muscle injuries in elite football: translating research into practice. British Journal of Sports Medicine. 52(10); 628-629. doi:10.1136/bjsports-2017-097573

Caparrós, T., Pujol, M., \& Salas, C. (2017). Pautas generales en el proceso de readaptación al entrenamiento después de una lesión deportiva. Apunts Medicina 
de lEsport. 52 (196); 129-172 doi: 10.1016/j.apunts.2017.02.002

Carling, C., Bloomfield, J., Nelsen, L., \& Reilly, T. (2008). The role of motion analysis in elite soccer: contemporary performance measurement techniques and work rate data. Sports Medicine, 38(10), 839-862.

Casais, L. (2008). Revisión de las estrategias para la prevención de lesiones en el deporte desde la actividad fisica. Apunts: Medicina de l'esport, ISSN 0213-3717, 43 (157), 30-40. doi:10.1016/S1886-6581(08)70066-5

Casamichana, D., \& Castellano, J. (2010). Time-motion, heart rate, perceptual and motor behaviour demands in small-sides soccer games: effects of pitch size. Journal of Sports $\quad$ Sciences, $28(14), \quad 1615-23$. doi:10.1080/02640414.2010.521168

Casamichana, D., \& Castellano, J. (2011). Demandas físicas en jugadores semiprofesionales de fútbol: ¿se entrena igual que se compite? Cultura, Ciencia y Deporte, 6(17), 121-127. doi:10.12800/ccd.v6i17.39

Casamichana, D., Castellano, J., \& Castagna, C. (2012). Comparing the Physical Demands of Friendly Matches and Small-Sided Games in Semiprofessional Soccer Players. Journal of Strength and Conditioning Research, 26(3), 837-843. doi:10.1519/JSC.0b013e31822a61 cf

Castagna, C., \& Barbero Álvarez, J. C. (2010). Physiological Demands of an Intermittent Futsal-Oriented High-Intensity Test. Journal of Strength and Conditioning Research, 24(9), 2322-2329. doi:10.1519/JSC.0b013e3181e347b9

Castellano, J., \& Álvarez, D. (2013). Uso defensivo del espacio de interacción en fútbol. (Defensive use of the interaction space in soccer). RICYDE. Revista Internacional de Ciencias Del Deporte, 9(32), 126-136. doi:10.5232/ricyde2013.03203 
Castellano, J., Blanco-Villaseñor, A., \& Álvarez, D. (2011). Contextual Variables and Time-Motion Analysis in Soccer. International Journal of Sports Medicine, 32(06), 415-421. doi:10.1055/s-0031-1271771

Castellano, J., \& Casamichana, D. (2013). Differences in the number of accelerations between small-sided games and friendly matches in soccer. Journal of Sports Science and Medicine. 12(1), 209-210.

Castellano, J., \& Casamichana, D. (2014). Deporte con dispositivos de posicionamiento global (GPS): Aplicaciones y limitaciones. Revista de Psicologia Del Deporte, 23(2), 355-364.

Castellano, J., Casamichana, D., Calleja-González, J., Román, J. S., \& Ostojic, S. M. (2011). Reliability and accuracy of $10 \mathrm{~Hz}$ GPS devices for short-distance exercise. Journal of Sports Science and Medicine, 10(1), 233-234.

Castellano, J., Fernández, J. C., Castillo, A., \& Casamichana, D. (2010). in a 7-a-Side Soccer Match. Portal, (Cv), 85-94.

Castillo, E. R., \& Lieberman, D. E. (2015). Lower back pain. Evolution, Medicine, and Public Health, 2015(1), 2-3. doi:10.1093/emph/eou034

Chan, O., Del Buono, A., Best, T. M., \& Maffulli, N. (2012). Acute muscle strain injuries: A proposed new classification system. Knee Surgery, Sports Traumatology, Arthroscopy, 20(11), 2356-2362. doi:10.1007/s00167-012-2118z

Chance-Larsen, K., Littlewood, C., \& Garth, A. (2010). Prone hip extension with lower abdominal hollowing improves the relative timing of gluteus maximus activation in relation to biceps femoris. Manual Therapy, 15(1), 61-65. doi:10.1016/j.math.2009.07.001

Charter, R. A. (2003). A Breakdown of Reliability Coefficients by Test Type and Reliability Method, and the Clinical Implications of Low Reliability. The 
Journal of General Psychology, 130(3), 290-304. doi:10.1080/00221300309601160

Chumanov, E. S., Heiderscheit, B. C., \& Thelen, D. G. (2007). The effect of speed and influence of individual muscles on hamstring mechanics during the swing phase of sprinting. Journal of Biomechanics, 40(16), 3555-3562. doi:10.1016/j.jbiomech.2007.05.026

Chumanov, E. S., Heiderscheit, B. C., \& Thelen, D. G. (2011). Hamstring musculotenon dynamics during stance and swing phases of high speed running. Medicine \& Science in Sports \& Exercise, 43(3), 525-532. doi:10.1249/MSS.0b013e3181f23fe8.Hamstring

Chumanov, E. S., Schache, A. G., Heiderscheit, B. C., \& Thelen, D. G. (2012). Hamstrings are most susceptible to injury during the late swing phase of sprinting. British Journal of Sports Medicine. 46(2):90. doi:10.1136/bjsports2011-090176

Chumanov, E. S., Wille, C. M., Michalski, M. P., \& Heiderscheit, B. C. (2012). Changes in muscle activation patterns when running step rate is increased. Gait \& Posture, 36(2), 231-235. doi:10.1016/j.gaitpost.2012.02.023

Cicchetti, D. (1994). Guidelines, Criteria, and Rules of Thumb for Evaluating Normed and Standardized Assessment Instrument in Psychology. Psychological Assessment (Vol. 6). doi:10.1037/1040-3590.6.4.284

Clark, R. A. (2008). Hamstring injuries: Risk assessment and injury prevention. Annals of the Academy of Medicine Singapore, 37(4), 341-346. doi: $10.3109 / 09593985.2016 .1138347$

Clemente, F., Owen, A., Serra-Olivares, J., Theodoros Nikolaidis, P., I van der Linden, C. M., \& Mendes, B. (2018). Characterization of the weekly external 
load profile of professional soccer teams from Portugal and the Netherlands. Ahead of print Journal of human Kinetics. doi:10.2478/hukin-2018-0054

Colyer, S. L., Nagahara, R., \& Salo, A. I. T. (2018). Kinetic demands of sprinting shift across the acceleration phase: novel analysis of entire force waveforms. Scandinavian Journal of Medicine \& Science in Sports. 28(7), 1784-1792. doi:10.1111/sms.13093

Comin, J., Malliaras, P., Baquie, P., Barbour, T., \& Connell, D. (2013). Return to Competitive Play After Hamstring Injuries Involving Disruption of the Central Tendon. The American Journal of Sports Medicine, 41(1), 111-115. doi:10.1177/0363546512463679

Coutts, A. J., \& Duffield, R. (2010). Validity and reliability of GPS devices for measuring movement demands of team sports. Journal of Science and Medicine in Sport, 13(1), 133-135. doi:10.1016/j.jsams.2008.09.015

Cook, G (2010). The joint by joint concept. In: Movement: Functional Movement Systems: Screening, Assesment and Corrective Strategies. California. Lotus publishing (pp 319-21).

Creighton, D., Shrier, I., Shultz, R., Meeuwisse, W. H., \& Matheson, G. O. (2010). Return-to-Play in Sport: A Decision-based Model. Clinical Journal of Sport Medicine, 20(5), 379-385. doi:10.1097/JSM.0b013e3181f3c0fe

Croisier, J.-L. (2004). Factors associated with recurrent hamstring injuries. Sports Medicine (Auckland, N.Z.), 34(10), 681-695.

Croisier, J.-L., Ganteaume, S., Binet, J., Genty, M., \& Ferret, J.-M. (2008). Strength Imbalances and Prevention of Hamstring Injury in Professional Soccer Players: A Prospective Study. The American Journal of Sports Medicine, 36(8), 14691475. doi:10.1177/0363546508316764 
Crossley, K. M., Zhang, W.-J., Schache, A. G., Bryant, A., \& Cowan, S. M. (2011). Performance on the Single-Leg Squat Task Indicates Hip Abductor Muscle Function. The American Journal of Sports Medicine, 39(4), 866-873. doi: $10.1177 / 0363546510395456$

Cruz-Ferreira, A., Marujo, A., Folgado, H., Gutierres Filho, P., \& Fernandes, J. (2015). Programas de exercício na prevenção de lesões em jogadores de futebol: uma revisão sistemática exercise programs in the preventing injuries in football players: a systematic review revisión sistemática. Revista Brasileira de Medicina do Esporte, 21 (3) 236-241. doi:10.1590/1517-86922015210302174

Cummins, C., Orr, R., O’Connor, H., \& West, C. (2013). Global positioning systems (GPS) and microtechnology sensors in team sports: a systematic review. Sports Medicine (Auckland, N.Z.), 43(10), 1025-1042. doi:10.1007/s40279-013-0069-2

Czuppon, S., Racette, B. A., Klein, S. E., \& Harris-Hayes, M. (2014). Variables associated with return to sport following anterior cruciate ligament reconstruction: a systematic review. British Journal of Sports Medicine, 48(5), 356-364. doi:10.1136/bjsports-2012-091786

Dalton, S. L., Kerr, Z. Y., \& Dompier, T. P. (2015). Epidemiology of Hamstring Strains in 25 NCAA Sports in the 2009-2010 to 2013-2014 Academic Years. The American Journal of Sports Medicine, 43(11), 2671-2679. doi:10.1177/0363546515599631

Dawson, B., Hopkinson, R., Appleby, B., Stewart, G., \& Roberts, C. (2004). Comparison of training activities and game demands in the Australian Football League. Journal of Science and Medicine in Sport, 7(3), 292-301.

De Hoyo, M., \& Aceña Rodríguez, A. (2017). Tecnologías aplicadas al fútbol. Sistemas de posicionamiento global (GPS). In Nuevas tecnologías aplicadas a la 
actividad fisica y el deporte (pp. 69-86). Pamplona (España). Aranzadi

De Hoyo, M., Cohen, D. D., Sanudo, B., Carrasco, L., Alvarez-Mesa, A., Del Ojo, J. J., .. Otero-Esquina, C. (2016). Influence of football match time-motion parameters on recovery time course of muscle damage and jump ability. Journal of Sports Sciences, 34(14), 1363-1370. doi:10.1080/02640414.2016.1150603

De Visser, H., Reijman, M., Heijboer, M., \& Bos, P. (2012). Risk factors of recurrent hamstring injuries: a systematic review. British Journal of Sports Medicine, 46(2), 124-130. doi:10.1136/bjsports-2011-090317

De Vos, R.-J., Reurink, G., Goudswaard, G.-J., Moen, M. H., Weir, A., \& Tol, J. L. (2014). Clinical findings just after return to play predict hamstring re-injury, but baseline MRI findings do not. British Journal of Sports Medicine, 48(18), 13771384. doi:10.1136/bjsports-2014-093737

Delgado-Rico, E., Carretero-Dios, H., \& Ruch, W. (2012). Content validity evidences in test development: An applied perspective. International Journal of Clinical and Health Psychology, 12(3), 449-460.

Dellal, A., Drust, B., \& Lago-Peñass, C. (2012). Variation of Activity Demands in Small-Sided Soccer Games. International Journal of Sports Medicine, 33(05), 370-375. doi:10.1055/s-0031-1295476

Dellal, A., Owen, A., Wong, D..P., Krustrup, P Van Exsel, M., \& Mallo, J.(2012). Technical and physical demands of small vs. large sided games in relation to playing position in elite soccer. Human Movement Science. 31 (4), 957-69. doi: 10.1016/j.humov.2011.08.013

Dello Iacono, A., \& Seitz, L. (2018). Hip thrust-based PAP effects on sprint performance of soccer players: heavy-loaded versus optimum-power development protocols. Journal of Sports Sciences. 36 (20), 2375-2382. 
doi:10.1080/02640414.2018.1458400

DeWitt, J., \& Vidale, T. (2014). Recurrent hamstring injury: consideration following operative and non-operative management. International Journal of Sports Physical Therapy, 9(6), 798-812.

Di Salvo, V., Baron, R., Gonzalez-Haro, C., Gormasz, C., Pigozzi, F., \& Bachl, N. (2010). Sprinting analysis of elite soccer players during European Champions League and UEFA Cup matches. Journal of Sports Sciences, 28(14), 1489-1494. doi:10.1080/02640414.2010.521166

Di Salvo, V., Baron, R., Tschan, H., Calderon Montero, F. J., Bachl, N., \& Pigozzi, F. (2007). Performance characteristics according to playing position in elite soccer. International Journal of Sports Medicine, 28(3), 222-227. doi:10.1055/s-2006924294

Di Salvo, V., Gregson, W., Atkinson, G., Tordoff, P., \& Drust, B. (2009). Analysis of high intensity activity in Premier League soccer. International Journal of Sports Medicine, 30(3), 205-212. doi:10.1055/s-0028-1105950

Dorn, T. W., Schache, A. G., \& Pandy, M. G. (2012). Muscular strategy shift in human running: dependence of running speed on hip and ankle muscle performance. The Journal of Experimental Biology, 215(Pt 11), 1944-1956. doi: $10.1242 /$ jeb. 064527

Dos'Santos, T., Thomas, C., Comfort, P., \& A. Jones, P. (2018). The Effect of Angle and Velocity on Change of Direction Biomechanics: An Angle-Velocity TradeOff. Sports Medicine. doi:10.1007/s40279-018-0968-3

Drawer, S., \& Fuller, C. W. (2002). Evaluating the level of injury in English professional football using a risk based assessment process. British Journal of Sports Medicine, 36(6), 446-51. doi:10.1136/BJSM.36.6.446 
Drezner, J. A. (2003). Practical management: hamstring muscle injuries. Clinical Journal of Sport Medicine : Official Journal of the Canadian Academy of Sport Medicine, 13(1), 48-52.

Duffield, R., Reid, M., Baker, J., \& Spratford, W. (2010). Accuracy and reliability of GPS devices for measurement of movement patterns in confined spaces for court-based sports. Journal of Science and Medicine in Sport, 13(5), 523-525. doi:10.1016/j.jsams.2009.07.003

Duhig, S., Shield, A. J., Opar, D., Gabbett, T., Ferguson, C., \& Williams, M. (2016). Effect of high-speed running on hamstring strain injury risk. British Journal of Sports Medicine, 50(24), $1536 \quad$ LP-1540. Retrieved from http://bjsm.bmj.com/content/50/24/1536.abstract

Dwyer, D. B., \& Gabbett, T. (2012). Global Positioning System Data Analysis: Velocity Ranges and a New Definition of Sprinting for Field Sport Athletes. Journal of Strength and Conditioning Research, 26(3), 818-824. doi:10.1519/JSC.0b013e3182276555

Edgecomb, S. J., \& Norton, K. I. (2006). Comparison of global positioning and computer-based tracking systems for measuring player movement distance during Australian Football. Journal of Science and Medicine in Sport, 9(1-2), 25-32. doi:10.1016/j.jsams.2006.01.003

Edouard, P., Branco, P., \& Alonso, J.-M. (2016). Muscle injuries and hamstring muscle injuries during the international athletics championships between 2007 and 2015. Annals of Physical and Rehabilitation Medicine, 59, e22. doi:10.1016/J.REHAB.2016.07.052

Edouard, P., Mendiguchia, J., Lahti, J., Arnal, P. J., Gimenez, P., Jiménez-Reyes, P., ... Morin, J.-B. (2018). Sprint Acceleration Mechanics in Fatigue Conditions: 
Compensatory Role of Gluteal Muscles in Horizontal Force Production and Potential Protection of Hamstring Muscles. Frontiers in Physiology, 9, 1706. doi:10.3389/fphys.2018.01706

Ehrmann, F. E., Duncan, C. S., Sindhusake, D., Franzsen, W. N., \& Greene, D. A. (2016). GPS and Injury Prevention in Professional Soccer. Journal of Strength and Conditioning $\quad$ Research, 360-367. doi:10.1519/JSC.0000000000001093

Eklund, D., Pulverenti, T., Bankers, S., Avela, J., Newton, R., Schumann, M., \& Häkkinen, K. (2014). Neuromuscular Adaptations to Different Modes of Combined Strength and Endurance Training. International Journal of Sports Medicine, 36(02), 120-129. doi:10.1055/s-0034-1385883

Ekstrand, J., Hagglund, M., \& Walden, M. (2011). Epidemiology of muscle injuries in professional football (soccer). The American Journal of Sports Medicine, 39(6), 1226-1232. doi:10.1177/0363546510395879

Ekstrand, J., Hägglund, M., \& Walden, M. (2011). Injury incidence and injury patterns in professional football: the UEFA injury study. British Journal of Sports Medicine, 45(7), 553-8. doi:10.1136/bjsm.2009.060582

Ekstrand, J., Healy, J. C., Walden, M., Lee, J. C., English, B., \& Hägglund, M. (2012). Hamstring muscle injuries in professional football: the correlation of MRI findings with return to play. British Journal of Sports Medicine, 46(2), 112-117. doi:10.1136/bjsports-2011-090155

Ekstrand, J., Walden, M., \& Hägglund, M. (2016). Hamstring injuries have increased by 4\% annually in men's professional football, since 2001: a 13-year longitudinal analysis of the UEFA Elite Club injury study. British Journal of Sports Medicine, 50(12), 731-737. doi:10.1136/bjsports-2015-095359 
Elgethun, K., Yost, M. G., Fitzpatrick, C. T. E., Nyerges, T. L., \& Fenske, R. A. (2006). Comparison of global positioning system (GPS) tracking and parentreport diaries to characterize children\&\#39;s time-location patterns. Journal Of Exposure Science And Environmental Epidemiology, 17, 196. Retrieved from http://dx.doi.org/10.1038/sj.jes.7500496

Elliott, M., Zarins, B., Powell, J., \& Kenyon, C. (2011). Hamstring Muscle Strains in Professional Football Players A 10-Year Review. The American journal of sports medicine, 39 (4), 843-50. doi:10.1177/0363546510394647

Engebretsen, A. H., Myklebust, G., Holme, I., Engebretsen, L., \& Bahr, R. (2010). Intrinsic Risk Factors for Hamstring Injuries Among Male Soccer Players. The American Journal of Sports Medicine, 38(6), 1147-1153. doi: $10.1177 / 0363546509358381$

Erickson, L. N., \& Sherry, M. A. (2017a). Rehabilitation and return to sport after hamstring strain injury. Journal of Sport and Health Science, 6(3), 262-270. doi:10.1016/j.jshs.2017.04.001

Erickson, L. N., \& Sherry, M. A. (2017b). Rehabilitation and return to sport after hamstring strain injury. Journal of Sport and Health Science, 6(3), 262-270. doi:10.1016/J.JSHS.2017.04.001

Feeley, B. T., Kennelly, S., Barnes, P., Muller, M. S., Kelly, B. T., Rodeo, S. A., \& Warren, R. F. (2008). Epidemiology of National Football League training camp injuries from 1998 to 2007. The American Journal of Sports Medicine, 36(8), 1597-1603. doi:10.1177/0363546508316021

Fernández-Gonzalo, R., Tesch, P. A., Linnehan, R. M., Kreider, R. B., Salvo, V. Di, Alomar, X., \& Rodas, G. (2016). Individual Muscle use in Hamstring Exercises by Soccer Players Assessed using Functional MRI. International Journal Sports 
Medicine, 37(7), 559-564. doi:10.1055/s-0042-100290

Forsdyke, D., Smith, A., Jones, M., \& Gledhill, A. (2016). Psychosocial factors associated with outcomes of sports injury rehabilitation in competitive athletes: a mixed studies systematic review. British Journal of Sports Medicine, 50(9), 53744. doi:10.1136/bjsports-2015-094850

Fort-Vanmeerhaeghe, A., Romero-Rodriguez, D., Lloyd, R. S., Kushner, A., \& Myer, G. D. (2016). Integrative Neuromuscular Training in Youth Athletes. Part II. Strength and Conditioning Journal, 38(4), 9-27. doi:10.1519/SSC.0000000000000234

Fort-Vanmeerhaeghe, A., \& Romero, D. (2013). Análisis de los factores de riesgo neuromusculares de las lesiones deportivas. Apunts Medicina de l'Esport, 48(179), 109-120.

Foster, C., Florhaug, J. A., Franklin, J., Gottschall, L., Hrovatin, L. A., Parker, S., ... Dodge, C. (2001). A new approach to monitoring exercise training. Journal of Strength and Conditioning Research, 15(1), 109-15.

Freckleton, G., Cook, J., \& Pizzari, T. (2014). The predictive validity of a single leg bridge test for hamstring injuries in Australian Rules Football Players. British Journal of Sports Medicine, 48(8), 713-717. doi:10.1136/bjsports-2013-092356

Freckleton, G., \& Pizzari, T. (2013). Risk factors for hamstring muscle strain injury in sport: a systematic review and meta-analysis. British Journal of Sports Medicine, 47(6), 351-358. doi:10.1136/bjsports-2011-090664

Frencken, W. G. P., Lemmink, K. A. P. M., \& Delleman, N. J. (2010). Soccer-specific accuracy and validity of the local position measurement (LPM) system. Journal of Science and Medicine in Sport, 13(6), 641-645.

doi:10.1016/j.jsams.2010.04.003 
Fuller, C. W., Ekstrand, J., Junge, A., Andersen, T. E., Bahr, R., Dvorak, J., ... Meeuwisse, W. H. (2006). Consensus statement on injury definitions and data collection procedures in studies of football (soccer) injuries. British Journal of Sports Medicine, 40(3), 193-201. doi:10.1136/bjsm.2005.025270

Gabbe, B. J., Bennell, K. L., Finch, C. F., Wajswelner, H., \& Orchard, J. (2006). Predictors of hamstring injury at the elite level of Australian football. Scandinavian Journal of Medicine and Science in Sports, 16(1), 7-13. doi:10.1111/j.1600-0838.2005.00441.x

Gabbett, T. (2010). GPS analysis of elite women's field hockey training and competition. Journal of Strength and Conditioning Research, 24(5), 1321-1324. doi:10.1519/JSC.0b013e3181ceebbb

Gabbett, T. (2017). The training-injury prevention paradox: should athletes be training smarter and harder? British Journal of Sports Medicine, 50(5), 273-80. doi:10.1136/bjsports-2015-095788

Gabbett, T., \& Jenkins, D. G. (2011). Relationship between training load and injury in professional rugby league players. Journal of Science and Medicine in Sport, 14(3), 204-209. doi:10.1016/j.jsams.2010.12.002

Gabbett, T., Jenkins, D. G., \& Abernethy, B. (2012). Physical demands of professional rugby league training and competition using microtechnology. Journal of Science and Medicine in Sport, 15(1), 80-86. doi:10.1016/j.jsams.2011.07.004

Gabbett, T., \& Mulvey, M. J. (2008). Time-motion analysis of small-sided training games and competition in elite women soccer players. Journal of Strength and Conditioning Research, 22(2), 543-552. doi:10.1519/JSC.0b013e3181635597

Gabbett, T., \& Whiteley, R. (2017). Two Training-Load Paradoxes: Can We Work 
Harder and Smarter, Can Physical Preparation and Medical Be Teammates? International Journal of Sports Physiology and Performance, 12(Suppl 2), S250-S2-54. doi:10.1123/ijspp.2016-0321

Gabriel, $\square$, Ramos, A., Arliani, G. G., Astur, D. C., De, A., Pochini, C., ... Cohen, M. (2017). Rehabilitation of hamstring muscle injuries: a literature review. Revista Brasileira de Ortopedia, 52, 11-16. doi:10.1016/j.rboe.2016.12.002

Galé-Ansodi, C., Castellano, J., \& Usabiaga, O. (2013). Descripción de las demandas fisicas en competición de jugadores de tenis en etapa de formación mediante la tecnología gps. En Arufe Giráldez, V (presidencia). IX Congreso Nacional de Ciencias del Deporte y la Educación Física. Pontevedra, 9-11 Mayo 2013. ISBN: $978-84-938695-3-3$

Garrett, W., Califf, J., \& H Bassett, F. (1984). Histochemical correlates of hamstring injuries. The American journal of sports medicine (Vol. 12). doi: $10.1177 / 036354658401200202$

Gaudino, P., Alberti, G., \& Iaia, F. M. (2014). Estimated metabolic and mechanical demands during different small-sided games in elite soccer players. Human Movement Science, 36, 123-133. doi:10.1016/j.humov.2014.05.006

Gaudino, P., Iaia, F. M., Alberti, G., Strudwick, A. J., Atkinson, G., \& Gregson, W. (2013). Monitoring training in elite soccer players: Systematic bias between running speed and metabolic power data. International Journal of Sports Medicine, 34(11), 963-968. doi:10.1055/s-0033-1337943

Girard, O., Méndez-Villanueva, A., \& Bishop, D. (2011). Repeated-sprint ability part I: Factors contributing to fatigue. Sports Medicine, 41(8), 673-694. doi:10.2165/11590550-000000000-00000

Gleason, B. H., Sams, M. L., Salley, J. T., Pustina, A. A., \& Stone, M. H. (2017). 
Global Positioning System Analysis of a High School Football Scrimmage. Journal of Strength and Conditioning Research, 31(8), 2183-2188. doi:10.1519/JSC.0000000000001691

Goldman, E. F., \& Jones, D. E. (2011). Interventions for preventing hamstring injuries: a systematic review. Physiotherapy, 97(2), 91-99. doi:10.1016/j.physio.2010.11.011

Gómez-Piqueras, P., Gonzalez-Rubio, J., Sainz de Baranda, P., Najera, A., \& de Baranda, S. P. (2018). Use of functional performance tests in sports: Evaluation proposal for football players in the rehabilitation phase. Turkish Journal of Physical Medicine and Rehabilitation, 64(2), 148-154. doi:10.5606/tftrd.2018.1462

Gómez-Piqueras, P., González-Víllora, S., Sainz de Baranda Andújar, D. M., \& Contreras-Jordán, R. O. (2017). Functional Assessment and Injury Risk in a Professional Soccer Team. Sports. 5(1), 9 . doi:10.3390/sports5010009

Gómez-Piriz, P. T., Jiménez-Reyes, P., \& Ruiz-Ruiz, C. (2011). Relation Between Total Body Load and Session Rating of Perceived Exertion in Professional Soccer Players. Journal of Strength and Conditioning Research, 25(8), 21002103. doi:10.1519/JSC.0b013e3181fb4587

Gray, A. J., Jenkins, D., Andrews, M. H., Taaffe, D. R., \& Glover, M. L. (2010). Validity and reliability of GPS for measuring distance travelled in field- based team sports. Journal of Sports Sciences, 28(12), 1319-1325. doi:10.1080/02640414.2010.504783

Gregson, W., Drust, B., Atkinson, G., \& Salvo, V. (2010). Match-to-Match Variability of High-Speed Activities in Premier League Soccer. International Journal of Sports Medicine, 31(04), 237-242. doi:10.1055/s-0030-1247546 
Guilford, J. P. (1954). Psychometric methods. Mcgraw Hill.

Hägglund, M. (2007). Epidemiology and prevention of football injuries (doctoral thesis) Linköping University. Linköping, Sweden.

Hagglund, M., Walden, M., \& Ekstrand, J. (2006). Previous injury as a risk factor for injury in elite football: a prospective study over two consecutive seasons. British Journal of Sports Medicine, 40(9), 767-772. doi:10.1136/bjsm.2006.026609

Hägglund, M., Walden, M., \& Ekstrand, J. (2013). Risk Factors for Lower Extremity Muscle Injury in Professional Soccer. The American Journal of Sports Medicine, 41(2), 327-335. doi:10.1177/0363546512470634

Hägglund, M., Walden, M., Magnusson, H., Kristenson, K., Bengtsson, H., \& Ekstrand, J. (2013). Injuries affect team performance negatively in professional football: an 11-year follow-up of the UEFA Champions League injury study. British Journal of Sports Medicine, 47(12), 738-42. doi:10.1136/bjsports-2013092215

Hallén, A., \& Ekstrand, J. (2014a). Return to play following muscle injuries in professional footballers. Journal of Sports Sciences, 32(13), 1229-1236. doi:10.1080/02640414.2014.905695

Hallén, A., \& Ekstrand, J. (2014b). Return to play following muscle injuries in professional footballers Return to play following muscle injuries in professional footballers. Journal of Sports Sciences, (May), 37-41. doi:10.1080/02640414.2014.905695

Halson, S. L. (2014). Monitoring training load to understand fatigue in athletes. Sports Medicine (Auckland, N.Z.), 44 Suppl 2(Suppl 2), S139-47. doi:10.1007/s40279-014-0253-z

Hamilton, B., Valle, X., Rodas, G., Til, L., Pruna Grive, R., Gutierrez Rincon, J. A., 
\& Tol, J. L. (2015). Classification and grading of muscle injuries: a narrative review. British Journal of Sports Medicine, 49(5), 306-306. doi:10.1136/bjsports-2014-093551

Hartwig, T. B., Naughton, G., \& Searl, J. (2011). Motion analyses of adolescent rugby union players: a comparison of training and game demands. Journal of Strength and Conditioning $\quad$ Research, 25(4), 966-972. doi:10.1519/JSC.0b013e3181d09e24

Hawkins, R. D., Hulse, M. A., Wilkinson, C., Hodson, A., \& Gibson, M. (2001). The association football medical research programme: an audit of injuries in professional football. British Journal of Sports Medicine, 35(1), 43 LP-47.

Hedrick, T., Bickman, L., \& Rog, D. (1993). Applied Research Design. Thousand Oaks, California. doi:10.4135/9781412983457

Hegyi, A., Csala, D., Péter, A., Finni, T., \& Cronin, N. J. (2019). High-density electromyography activity in various hamstring exercises. Scandinavian Journal of Medicine \& Science in Sports, 29(1), 34-43. doi:10.1111/sms.13303

Heiderscheit, B. C., Hoerth, D. M., Chumanov, E. S., Swanson, S. C., Thelen, B. J., \& Thelen, D. G. (2005). Identifying the time of occurrence of a hamstring strain injury during treadmill running: A case study. Clinical Biomechanics, 20(10), 1072-1078. doi:10.1016/j.clinbiomech.2005.07.005

Heiderscheit, B. C., Sherry, M. A., Silder, A., Chumanov, E. S., \& Thelen, D. G. (2010). Hamstring Strain Injuries: Recommendations for Diagnosis, Rehabilitation, and Injury Prevention. Journal of Orthopaedic \& Sports Physical Therapy, 40(2), 67-81. doi:10.2519/jospt.2010.3047

Henderson, B., Cook, J., Kidgell, D. J., \& Gastin, P. B. (2015). Game and Training Load Differences in Elite Junior Australian Football. Journal of Sports Science 
\& Medicine, 14(3), 494-500.

Henderson, G., Barnes, C. A., \& Portas, M. D. (2010). Factors associated with increased propensity for hamstring injury in English Premier League soccer players. Journal of Science and Medicine in Sport, 13(4), 397-402. doi:10.1016/j.jsams.2009.08.003

Herzog, W., \& Read, L. J. (1993). Lines of action and moment arms of the major force-carrying structures crossing the human knee joint. Journal of Anatomy, 182 (2), 213-30.

Hickey, J. T., Shield, A. J., Williams, M., \& Opar, D. A. (2014). The financial cost of hamstring strain injuries in the Australian Football League. British Journal of Sports Medicine, 48(8), 729-730. doi:10.1136/bjsports-2013-092884

Hickey, J. T., Timmins, R. G., Maniar, N., Williams, M., \& Opar, D. A. (2017). Criteria for Progressing Rehabilitation and Determining Return-to-Play Clearance Following Hamstring Strain Injury: A Systematic Review. Sports Medicine, 47(7), 1375-1387. doi:10.1007/s40279-016-0667-x

Higashihara, A., Nagano, Y., Ono, T., \& Fukubayashi, T. (2015). Differences in activation properties of the hamstring muscles during overground sprinting. Gait \& Posture, 42(3), 360-364. doi:10.1016/j.gaitpost.2015.07.002

Higashihara, A., Nagano, Y., Ono, T., \& Fukubayashi, T. (2018). Differences in hamstring activation characteristics between the acceleration and maximumspeed phases of sprinting. Journal of Sports Sciences, 36(12), 1313-1318. doi:10.1080/02640414.2017.1375548

Higashihara, A., Ono, T., Kubota, J., Okuwaki, T., \& Fukubayashi, T. (2010). Functional differences in the activity of the hamstring muscles with increasing running speed. Journal of Sports Sciences, 28(10), 1085-1092. 
doi:10.1080/02640414.2010.494308

Hill-Haas, S. V, Dawson, B., Impellizzeri, F. M., \& Coutts, A. J. (2011). Physiology of small-sided games training in football: a systematic review. Sports Medicine, 41(3), 199-220. doi:10.2165/11539740-000000000-00000

Hopkins, W. G., Marshall, S. W., Batterham, A. M., \& Hanin, J. (2009). Progressive statistics for studies in sports medicine and exercise science. Medicine and Science in Sports and Exercise, 41(1), 3-13. doi:10.1249/MSS.0b013e31818cb278

Hoppe, M. W., Baumgart, C., Polglaze, T., \& Freiwald, J. (2018). Validity and reliability of GPS and LPS for measuring distances covered and sprint mechanical properties in team sports. PloS One, 13(2), e0192708. doi:10.1371/journal.pone.0192708

Iaia, F. M., Fiorenza, M., Larghi, L., Alberti, G., Millet, G. P., \& Girard, O. (2017). Short- or long-rest intervals during repeated-sprint training in soccer? PloS One, 12(2), e0171462. doi:10.1371/journal.pone.0171462

Impellizzeri, F. M., Rampinini, E., Coutts, A. J., Sassi, A., \& Marcora, S. M. (2004). Use of RPE-based training load in soccer. Medicine and Science in Sports and Exercise, 36(6), 1042-7.

Järvinen, T. . (1977). Immobilization effect on the tensile properties of striated muscle: an experimental study in the rat. Archives of Physical Medicine and Rehabilitation, 58(3), 123-7.

Järvinen, T. ., Kääriäinen, M., Äärimaa, V., Vaittinen, S., \& Kalimo, H. (2007). Muscle injuries: optimising recovery. Best Practice \& Research Clinical Rheumatology, 21(2), 317-331. doi:10.1016/j.berh.2006.12.004

Järvinen, T. ., Kääriäinen, M., \& Kalimo, H. (2005). Muscle Injuries. The American 
Journal of Sports Medicine, 33(5), 745-764. doi:10.1177/0363546505274714

Järvinen, T. ., \& Kalimo, H. (2013). Regeneration of injured skeletal muscle after the injury. Muscles, Ligaments and Tendons Journal, 3(4), 337-45. doi:10.11138/mltj/2013.3.4.337

Jaspers, A., Brink, M. S., Probst, S. G. M., Frencken, W. G. P., \& Helsen, W. F. (2017). Relationships Between Training Load Indicators and Training Outcomes in Professional Soccer. Sports Medicine, 47(3), 533-544. doi:10.1007/s40279016-0591-0

Jenkins, M. T., Gustitus, R., Iosia, M., Kicklighter, T., \& Sasaki, Y. (2017). Correlation between the Functional Movement Screen and Hip Mobility in NCAA Division II Athletes. International Journal of Exercise Science, 10(4), $541-549$.

Jennings, D., Cormack, S., Coutts, A. J., Boyd, L., \& Aughey, R. J. (2010a). The validity and reliability of GPS units for measuring distance in team sport specific running patterns. International Journal of Sports Physiology and Performance, $5(3), 328-41$.

Jennings, D., Cormack, S., Coutts, A. J., Boyd, L. J., \& Aughey, R. J. (2010b). Variability of GPS units for measuring distance in team sport movements. International Journal of Sports Physiology and Performance, 5(4), 565-9.

Jiménez-Rubio, S., Navandar, A., Rivilla-García, J., \& Paredes-Hernández, V. (2018). Validity of an on-Field Readaptation Program Following a Hamstring Injury in Professional Soccer. Journal of Sport Rehabilitation, 1-20. doi:10.1123/jsr.2018-0203

Johnston, R., Gibson, N. V, Twist, C., Gabbett, T., MacNay, S. A., \& MacFarlane, N. G. (2013). Physiological responses to an intensified period of rugby league 
competition. Journal of Strength and Conditioning Research, 27(3), 643-54. doi:10.1519/JSC.0b013e31825bb469

Johnston, R., Watsford, M. L., Pine, M. J., Spurrs, R. W., \& Sporri, D. (2013). Assessment of $5 \mathrm{~Hz}$ and $10 \mathrm{~Hz}$ GPS units for measuring athlete movement demands. International Journal of Performance Analysis in Sport, 13(1) 262-274. doi:10.1080/24748668.2013.11868646

Jonathan, N., Russell, M., Shearer, D., Cook, C., \& Kilduff, L. (2017). Predictors Of Linear And Multidirectional Acceleration In Elite Soccer Players. Journal of Strength and Conditioning Research, 1. doi:10.1519/JSC.0000000000001897

Junge, A., \& Dvorak, J. (2013). Injury surveillance in the World Football Tournaments 1998-2012. British Journal of Sports Medicine, 47(12), 782-8. doi:10.1136/bjsports-2013-092205

Junge, A., \& Dvořák, J. (2015). Football injuries during the 2014 FIFA World Cup. British Journal of Sports Medicine, 49(9), 599-602. doi:10.1136/bjsports-2014094469

Kadi, F., Schjerling, P., Andersen, L. L., Charifi, N., Madsen, J. L., Christensen, L. R., \& Andersen, J. L. (2004). The effects of heavy resistance training and detraining on satellite cells in human skeletal muscles. The Journal of Physiology, 558(3), 1005-1012. doi:10.1113/jphysiol.2004.065904

Kapandji, A. I. (2010). Fisiologia articular / miembros inferiores / lower limb. Editorial Medica Panamericana.

Kellis, E., Katis, A., \& Gissis, I. (2004). Knee biomechanics of the support leg in soccer kicks from three angles of approach. Medicine and Science in Sports and Exercise, 36(6), 1017-1028.

Kilcoyne, K. G., Dickens, J. F., Keblish, D., Rue, J.-P., \& Chronister, R. (2011). 
Outcome of Grade I and II Hamstring Injuries in Intercollegiate Athletes. Sports Health: A Multidisciplinary Approach, 3(6), 528-533. doi: $10.1177 / 1941738111422044$

King, E., Franklyn-Miller, A., Richter, C., O’Reilly, E., Doolan, M., Moran, K., ... Falvey, É. (2018). Clinical and biomechanical outcomes of rehabilitation targeting intersegmental control in athletic groin pain: prospective cohort of 205 patients. British Journal of Sports Medicine, 52(16), 1054-1062.

Klausen, K., Andersen, L. L., \& PELLE, I. (1981). Adaptive changes in work capacity, skeletal muscle capillarization and enzyme levels during training and detraining. Acta Physiologica Scandinavica, 113(1), 9-16. doi:10.1111/j.17481716.1981.tb06854.x

Koulouris, G., \& Connell, D. (2003). Evaluation of the hamstring muscle complex following acute injury. Skeletal Radiology, 32(10), 582-589. doi:10.1007/s00256-003-0674-5

Krustrup, P., \& Krustrup, B. R. (2018). Football is medicine: it is time for patients to play! British Journal of Sports Medicine, bjsports-2018-099377. doi:10.1136/bjsports-2018-099377

Labsy, Z., Collomp, K., Frey, A., \& De Ceaurriz, J. (2004). Assessment of maximal aerobic velocity in soccer players by means of an adapted Probst field test. The Journal of Sports Medicine and Physical Fitness, 44(4), 375-82.

Lacome, M., Simpson, B. M., Cholley, Y., Lambert, P., \& Buchheit, M. (2018). Small-Sided Games in Elite Soccer: Does One Size Fit All? International Journal of Sports Physiology and Performance, 13(5), 568-576. doi:10.1123/ijspp.2017-0214

Lago, C., Casais, L., Domínguez, E., \& Sampaio, J. (2010). The effects of situational 
variables on distance covered at various speeds in elite soccer. European Journal of Sport Science, 10(2), 103-109. doi:10.1080/17461390903273994.

Lalín, C. \& Peirau, X. (2011). La reeducación funcional deportiva. En Nacleiro, F. Entrenamiento deportivo: fundamentos y aplicaciones en diferentes deportes, 27; 419-429. Editorial Médica Panamericana. Madrid.

Lalín Novoa, C. (2008a). La readaptación lesional (I parte): fundamentación y contextualización. RED: Revista de Entrenamiento Deportivo, XXII(2), 27-35.

Lalín Novoa, C. (2008b). La readaptación lesional (II parte): fundamentación y contextualización. RED: Revista de Entrenamiento Deportivo, XXII(3), 29-37.

Lempainen, L., Banke, I. J., Johansson, K., Brucker, P. U., Sarimo, J., Orava, S., \& Imhoff, A. B. (2015). Clinical principles in the management of hamstring injuries. Knee Surgery, Sports Traumatology, Arthroscopy, 23(8), 2449-2456. doi:10.1007/s00167-014-2912-x

Liebenson, C., Karpowicz, A. M., Brown, S. H. M., Howarth, S. J., \& McGill, S. M. (2009). The Active Straight Leg Raise Test and Lumbar Spine Stability. $P M \& R$, 1(6), 530-535. doi:10.1016/j.pmrj.2009.03.007

Little, T., \& Williams, A. G. (2005). Specificity of Acceleration, Maximum Speed, and Agility in Professional Soccer Players. The Journal of Strength and Conditioning Research, 19(1), 76-78. doi:10.1519/14253.1

Lubetzky-Vilnai, A., Carmeli, E., \& Katz-Leurer, M. (2009). Prevalence of injuries among young adults in sport centers: relation to the type and pattern of activity. Scandinavian Journal of Medicine \& Science in Sports, 19(6), 828-833. doi:10.1111/j.1600-0838.2008.00854.x

Luisa, C. R. M., \& Francisco, R. R. J. (2004). La teoría de la complejidad y su influencia en la escuela. En Revista de Teoría y Didáctica de las Ciencias 
Sociales, 9, 131-141.

MacLeod, H., Morris, J., Nevill, A., \& Sunderland, C. (2009). The validity of a nondifferential global positioning system for assessing player movement patterns in field hockey. Journal of Sports Sciences, 27(2), 121-128. doi:10.1080/02640410802422181

Maeo, S., Shan, X., Otsuka, S., Kanehisa, H., \& Kawakami, Y. (2018). Neuromuscular Adaptations to Work-matched Maximal Eccentric vs Concentric Training. Medicine and Science in Sports and Exercise, 50(8), 1629-1640. doi:10.1249/MSS.0000000000001611

Malliaropoulos, N., Panagiotis, T., Jurdan, M., Vasilis, K., Debasish, P., Peter, M., \& Tsapralis, K. (2015). Muscle and intensity based hamstring exercise classification in elite female track and field athletes: implications for exercise selection during rehabilitation. Open Access Journal of Sports Medicine, 209. doi:10.2147/OAJSM.S79189

Malliaropoulos, N., Papacostas, E., Kiritsi, O., Papalada, A., Gougoulias, N., \& Maffulli, N. (2010). Posterior thigh muscle injuries in elite track and field athletes. The American Journal of Sports Medicine, 38(9), 1813-1819. doi: $10.1177 / 0363546510366423$

Mallo, J., Mena, E., Nevado, F., \& Paredes, V. (2015). Physical Demands of TopClass Soccer Friendly Matches in Relation to a Playing Position Using Global Positioning System Technology. Journal of Human Kinetics, 47(1), 179-188. doi:10.1515/hukin-2015-0073

Malone, S., Owen, A., Mendes, B., Hughes, B., Collins, K., \& Gabbett, T. (2017). High-speed running and sprinting as an injury risk factor in soccer: Can welldeveloped physical qualities reduce the risk? Journal of Science and Medicine in 
Sport, 21(3), 257-262. doi:10.1016/j.jsams.2017.05.016

Malone, S., Owen, A., Newton, M., Mendes, B., Collins, K. C., \& Gabbett, T. (2016). No Title. J Sci Med Sport, 20 (null), 561.

Manouras, N., Papanikolaou, Z., Karatrantou, K., Kouvarakis, P., \& Gerodimos, V. (2016). The efficacy of vertical vs. horizontal plyometric training on speed, jumping performance and agility in soccer players. International Journal of Sports Science \& Coaching, 11(5), 702-709. doi:10.1177/1747954116667108

Manzi, V., Bovenzi, A., Franco Impellizzeri, M., Carminati, I., \& Castagna, C. (2013). Individual Training-Load and Aerobic-Fitness Variables in Premiership Soccer Players During the Precompetitive Season. Journal of Strength and Conditioning Research, 27(3), 631-636. doi:10.1519/JSC.0b013e31825dbd81

Manzi, V., Iellamo, F., Impellizzeri, F., D’ottavio, S., \& Castagna, C. (2009). Relation between Individualized Training Impulses and Performance in Distance Runners. Medicine \& Science in Sports \& Exercise, 41(11), 2090-2096. doi:10.1249/MSS.0b013e3181a6a959

Martínez-valencia, M. A., Romero-arenas, S., \& Elvira, J. L. L. (2015). Effects of Sled Towing on Peak Force, the Rate of Force Development and Sprint Performance During the Acceleration Phase. Journal Human Kinetics. 46(2), 139-148. doi:10.1515/hukin-2015-0042.

Mason, D. L., Dickens, V. A., \& Vail, A. (2012). Rehabilitation for hamstring injuries. The Cochrane Database of Systematic Reviews, 12, CD004575. doi:10.1002/14651858.CD004575.pub3

McCall, A., Carling, C., Nedelec, M., Davison, M., Le Gall, F., Berthoin, S., \& Dupont, G. (2014). Risk factors, testing and preventive strategies for non-contact injuries in professional football: current perceptions and practices of 44 teams 
from various premier leagues. British Journal of Sports Medicine, 48, 1352-1357. doi:10.1136/bjsports-2014-093439.

Méndez-Villanueva, A., Suárez-Arrones, L., Rodas, G., Fernandez-Gonzalo, R., Tesch, P., Linnehan, R., ... Di Salvo, V. (2016). MRI-Based Regional Muscle Use during Hamstring Strengthening Exercises in Elite Soccer Players. PLOS ONE, 11(9), e0161356. doi:10.1371/journal.pone.0161356

Mendiguchia, J., Alentorn-Geli, E., \& Brughelli, M. (2012). Hamstring strain injuries: are we heading in the right direction? British Journal of Sports Medicine, 46(2), 81-85. doi:10.1136/bjsm.2010.081695

Mendiguchia, J., \& Brughelli, M. (2011). A return-to-sport algorithm for acute hamstring injuries. Physical Therapy in Sport: Official Journal of the Association of Chartered Physiotherapists in Sports Medicine, 12(1), 2-14. doi:10.1016/j.ptsp.2010.07.003

Mendiguchia, J., Edouard, P., Samozino, P., Brughelli, M., Cross, M., Ross, A., ... Morin, J. B. (2016). Field monitoring of sprinting power-force-velocity profile before, during and after hamstring injury: two case reports. Journal of Sports Sciences, 34(6), 535-541. doi:10.1080/02640414.2015.1122207

Mendiguchia, J., Martinez-Ruiz, E., Edouard, P., Morin, J. B., Martinez-Martinez, F., Idoate, F., \& Méndez-Villanueva, A. (2017). A Multifactorial, Criteria-based Progressive Algorithm for Hamstring Injury Treatment. Medicine and Science in Sports and Exercise, 49(7), 1482-1492. doi:10.1249/MSS.0000000000001241

Mendiguchia, J., Martinez-Ruiz, E., Morin, J. B., Samozino, P., Edouard, P., Alcaraz, P. E., .. Méndez-Villanueva, A. (2015). Effects of hamstring-emphasized neuromuscular training on strength and sprinting mechanics in football players. Scandinavian Journal of Medicine \& Science in Sports, 25(6), e621-e629. 
doi: $10.1111 / \mathrm{sms} .12388$

Mendiguchia, J., Samozino, P., Martinez-Ruiz, E., Brughelli, M., Schmikli, S., Morin, J.-B., \& Méndez-Villanueva, A. (2014). Progression of Mechanical Properties during On-field Sprint Running after Returning to Sports from a Hamstring Muscle Injury in Soccer Players. International Journal of Sports Medicine, 35(08), 690-695. doi:10.1055/s-0033-1363192

Miñano-Espin, J., Casais, L., Lago-Peñas, C., \& Gómez-Ruano, M. A. (2017). High Speed Running and Sprinting Profiles of Elite Soccer Players. Journal of Human Kinetics, 58, 169-176. doi:10.1515/hukin-2017-0086

Monajati, A., Larumbe-Zabala, E., Goss-Sampson, M., \& Naclerio, F. (2014). The Effectiveness of Injury Prevention Programs to Modify Risk Factors for NonContact Anterior Cruciate Ligament and Hamstring Injuries in Uninjured Team Sports Athletes: A Systematic Review. PloS one, 11(5), e0155272. doi:10.1371/journal.pone.0155272

Morin, J. B., Gimenez, P., Edouard, P., Arnal, P., Jiménez-Reyes, P., Samozino, P., ... Mendiguchia, J. (2015). Sprint Acceleration Mechanics: The Major Role of Hamstrings in Horizontal Force Production. Frontiers in Physiology, 6, 404. doi:10.3389/fphys.2015.00404

Mueller-Wohlfahrt, H.-W., Haensel, L., Mithoefer, K., Ekstrand, J., English, B., McNally, S., ... Ueblacker, P. (2013). Terminology and classification of muscle injuries in sport: The Munich consensus statement. British Journal of Sports Medicine, 47(6), 342-350. doi:10.1136/bjsports-2012-091448

Mueller, P. (2007). Exercise training and sympathetic nervous system activity: Evidence for physical activity dependent neural plasticity. Clinical and experimental pharmacology \& physiology (Vol. 34). doi:10.1111/j.1440- 
1681.2007.04590.x

Mujika, I. (2000). Detraining: Loss of Training-Induced Physiological and Performance Adaptations. Part I. Sports Medicine, 30(2), 79-87.

Mujika, I., \& Padilla, S. (2001). Muscular characteristics of detraining in humans. Medicine and Science in Sports and Exercise, 33(8), 1297-1303. doi:10.1097/00005768-200108000-00009

Mujika, I., Spencer, M., Santisteban, J., Goiriena, J. J., \& Bishop, D. (2009). Agerelated differences in repeated-sprint ability in highly trained youth football players. Journal of Sports Sciences, 27(14), 1581-1590. doi:10.1080/02640410903350281

Navandar, A., Garcia, C., Veiga, S., Torres, G., Chorro, D., \& Navarro, E. (2015). Effect of previous hamstring injury and limb dominance on kicking biomechanics in elite female soccer players. En: "International Society for Biomechanics in Sports", 2015, Poitiers. France.

Navandar, A., Gulino, M., Antonio, R., \& Navarro, E. (2013). Effect of hamstring injuries on kicking in soccer using inverse dynamics. Biomecánica, 21(1), 7-19. https://upcommons.upc.edu/handle/2099/15651

Neto, T., Jacobsohn, L., Carita, A. I., \& Oliveira, R. (2015). Reliability of the ActiveKnee-Extension and Straight-Leg-Raise Tests in Subjects With Flexibility Deficits. Journal of Sport Rehabilitation, 24(4), jsr.2014-0220. doi:10.1123/jsr.2014-0220

Neumann, D. A., Rowan, E. E., \& González del Campo Román, P. (2007). Cinesiología del sistema musculoesquelético : fundamentos de la rehabilitación fisica. Paidotribo.

Noya, J., Gómez-Carmona, P. M., Gracia-Marco, L., Moliner-Urdiales, D., \& Sillero- 
Quintana, M. (2014). Epidemiology of injuries in First Division Spanish football. Journal of Sports Sciences, $\quad 32(13), \quad$ 1263-1270. doi: $10.1080 / 02640414.2014 .884720$

Noya, J., \& Sillero, M. (2011). Incidencia lesional en el fútbol profesional español a lo largo de una temporada: días de baja por lesión. Apunts Medicina de l'Esport. 47(176), 113-178. doi:10.1016/j.apunts.2011.10.001

Nunome, H., IkegamI, Y., Kozakai, R., Apriantono, T., \& Sano, S. (2006). Segmental dynamics of soccer instep kicking with the preferred and non-preferred leg. Journal of Sports Sciences, 24(5), 529-541. doi:10.1080/02640410500298024

Olmo, J., Aramberri, M., Almaraz, C., Nayler, J., \& Requena, B. (2018). Successful conservative treatment for a subtotal proximal avulsion of the rectus femoris in an elite soccer player. Physical therapy in sport. 33, 62-69. doi:10.1016/j.ptsp.2018.07.004

Opar, D. A., Piatkowski, T., Williams, M., \& Shield, A. J. (2013). A Novel Device Using the Nordic Hamstring Exercise to Assess Eccentric Knee Flexor Strength: A Reliability and Retrospective Injury Study. Journal of Orthopaedic \& Sports Physical Therapy, 43(9), 636-640. doi:10.2519/jospt.2013.4837

Opar, D. A., Williams, M., \& Shield, A. J. (2012a). Hamstring strain injuries: factors that lead to injury and re-injury. Sports Medicine, 42(3), 209-226. doi:10.2165/11594800-000000000-00000

Opar, D. A., Williams, M., \& Shield, A. J. (2012b). Hamstring Strain Injuries. Sports Medicine, 42(3), 209-226. doi:10.2165/11594800-000000000-00000

Opar, D. A., Williams, M., Timmins, R. G., Hickey, J. T., Duhig, S. J., \& Shield, A. J. (2015). Eccentric hamstring strength and hamstring injury risk in Australian footballers. Medicine and Science in Sports and Exercise, 47(4). 
doi:10.1249/MSS.0000000000000465

Orchard, J., Kountouris, A., \& Sims, K. (2016). Incidence and prevalence of elite male cricket injuries using updated consensus definitions. Open Access Journal of Sports Medicine, 7, 187-194. doi:10.2147/OAJSM.S117497

Orchard, J., \& Seward, H. (2002). Epidemiology of injuries in the Australian Football League, seasons 1997-2000. British Journal of Sports Medicine, 36(1), 39-44. doi:10.1136/BJSM.36.1.39

Orchard, J., \& Seward, H. (2004). AFL injury report 2002. Sport Health, 21(1), 45-51.

Owen, A. L., Djaoui, L., Newton, M., Malone, S., \& Mendes, B. (2017). A contemporary multi-modal mechanical approach to training monitoring in elite professional soccer. Science and Medicine in Football, 1(3), 216-221. doi: $10.1080 / 24733938.2017 .1334958$

Pacheco-Arajol, L., \& Pujol Marzo, M. (2013). Readaptación al esfuerzo después de una lesión muscular. In Lesiones musculares en el deporte (pp. 101-106). Madrid. Panamericana.

Padua, D. A., Frank, B., Donaldson, A., de la Motte, S., Cameron, K. L., Beutler, A. I., ... Marshall, S. W. (2014). Seven Steps for Developing and Implementing a Preventive Training Program. Clinics in Sports Medicine, 33(4), 615-632. doi:10.1016/j.csm.2014.06.012

Padulo, J., Attene, G., Ardigò, L. P., Bragazzi, N. L., Maffulli, N., Zagatto, A. M., \& Dello Iacono, A. (2017). Can a Repeated Sprint Ability Test Help Clear a Previously Injured Soccer Player for Fully Functional Return to Activity? A Pilot Study. Clinical Journal of Sport Medicine, 27(4), 361-368. doi:10.1097/JSM.0000000000000368 
Paredes Hernández, V., Martos Varela, S.\& Romero, B. (2011). Propuesta de readaptación para la rotura del ligamento cruzado anterior en fútbol. Revista Internacional de Medicina y Ciencias de la Actividad FÃsica y del Deporte, 11 (43), 573-591.

Penfield, R., \& Giacobbi, Jr., P. R. (2004). Applying a Score Confidence Interval to Aiken's Item Content-Relevance Index. Measurement in Physical Education and Exercise Science, 8(4), 213-225. doi:10.1207/s15327841mpee0804_3

Perroca, M. G. (2011). Development and Content Validity of the New Version of a Patient Classification Instrument. Rev. Latino-Americana de Enfermagem. 19(1), 58-66. doi: 10.1590/S0104-11692011000100009.

Petersen, C., Pyne, D., Portus, M., \& Dawson, B. (2009). Validity and reliability of GPS units to monitor cricket-specific movement patterns. International Journal of Sports Physiology and Performance, 4(3), 381-393.

Petersen, Thorborg, K., Nielsen, M. B., Budtz-Jørgensen, E., \& Hölmich, P. (2011). Preventive Effect of Eccentric Training on Acute Hamstring Injuries in Men's Soccer. The American Journal of Sports Medicine, 39(11), 2296-2303. doi: $10.1177 / 0363546511419277$

Pickering, C., \& Kiely, J. (2018). Hamstring injury prevention: A role for genetic information? Medical Hypotheses, 119, 58-62. doi:10.1016/j.mehy.2018.07.011

Pollock, N., Patel, A., Chakraverty, J., Suokas, A., James, S. L. J., \& Chakraverty, R. (2016). Time to return to full training is delayed and recurrence rate is higher in intratendinous ('c') acute hamstring injury in elite track and field athletes: clinical application of the British Athletics Muscle Injury Classification. British Journal of Sports Medicine, 50(5), 305-310. doi:10.1136/bjsports-2015-094657

Portas, M. D., Harley, J. A., Barnes, C., \& Rush, C. J. (2010). The validity and 
reliability of $1-\mathrm{Hz}$ and $5-\mathrm{Hz}$ global positioning systems for linear, multidirectional, and soccer-specific activities. International Journal of Sports Physiology and Performance, 5(4), 448-58. doi:10.1123/ijspp.5.5.448.

Pratas, J., Volossovitch, A., \& Ferreira, A. P. (2012). The Effect of Situational Variables on Teams' Performance in Offensive Sequences Ending in a Shot on Goal. A Case Study. The Open Sports Sciences Journal, 5, 193-199.

Price, R. J., Hawkins, R. D., Hulse, M. A., \& Hodson, A. (2004). The Football Association medical research programme: an audit of injuries in academy youth football. British Journal of Sports Medicine, 38(4), 466-471. doi:10.1136/bjsm.2003.005165

Prior, M., Guerin, M., \& Grimmer, K. (2009). An evidence-based approach to hamstring strain injury: a systematic review of the literature. Sports Health, 1(2), 154-64. doi:10.1177/1941738108324962

Proske, U., Morgan, D., Brockett, C., \& Percival, P. (2004). Identifying athletes at risk of hamstring strains and how to protect them. Clinical and Experimental Pharmacology and Physiology, 31(8), 546-550. doi:10.1111/j.14401681.2004.04028.x

Raabe, M. E., \& Chaudhari, A. M. W. (2018). Biomechanical consequences of running with deep core muscle weakness. Journal of Biomechanics, 67, 98-105. doi:10.1016/j.jbiomech.2017.11.037

Räisänen, A. M., Pasanen, K., Krosshaug, T., Vasankari, T., Kannus, P., Heinonen, A., ... Parkkari, J. (2018). Association between frontal plane knee control and lower extremity injuries: a prospective study on young team sport athletes. BMJ Open Sport \&amp;Amp; Exercise Medicine, 4(1).

Rampinini, E., Sassi, A., Morelli, A., Mazzoni, S., Fanchini, M., \& Coutts, A. J. 
(2009). Repeated-sprint ability in professional and amateur soccer players. Applied Physiology, Nutrition, and Metabolism, 34(6), 1048-1054. doi:10.1139/H09-111.

Randers, M. B., Mujika, I., Hewitt, A., Santisteban, J., Bischoff, R., Solano, R., ... Mohr, M. (2010). Application of four different football match analysis systems: A comparative study. Journal of Sports Sciences, 28(2), 171-182. doi:10.1080/02640410903428525

Redwood-Brown, A., Cranton, W., \& Sunderland, C. (2012). Validation of a RealTime Video Analysis System for Soccer. International Journal of Sports Medicine, 33(08), 635-640. doi:10.1055/s-0032-1306326

Reid, L. C., Cowman, J. R., Green, B. S., \& Coughlan, G. F. (2013). Return to play in elite rugby union: application of global positioning system technology in returnto-running programs. Journal of Sport Rehabilitation, 22(2), 122-129.

Reilly, T., \& Thomas V. (1976). A motion analysis of work-rate in different positional roles in professional football match-play. J Human Movement Studies, 2, 87-97.

Reiman, M. P., \& Lorenz, D. S. (2011). Integration of strength and conditioning principles into a rehabilitation program. International Journal of Sports Physical Therapy, 6(3), 241-253.

Rivilla-García, J., Calvo, L. C., Jiménez, S., Muñoz, A., Bautista, I. J., Tillaar, R. Van Den, \& Navandar, A. (2018). Characteristics of very high intensity runs of soccer players in relation to their playing position and playing half in the 201314 spanish La liga season. Ahead of print Journal of human Kinetics. doi:10.2478/hukin-2018-0058.

Rodero, C. L., Molina Díaz, A. I., Fernández Guerrero, M., Ángel, M., \& Duque, R. 
(2015). Análisis de la fiabilidad y validez de un cuestionario docente. Actas de las XXI Jornadas de la Enseñanza Universitaria de la informática. Andorra

Roecker, K., Mahler, H., Heyde, C., Röll, M., \& Gollhofer, A. (2017). The relationship between movement speed and duration during soccer matches. PloS one, 12(7). doi: 10.1371/journal.pone.0181781.

Røksund, O. D., Kristoffersen, M., Bogen, B. E., Wisnes, A., Engeseth, M. S., Nilsen, A. K., ... Gundersen, H. (2017). Higher drop in speed during a repeated sprint test in soccer players reporting former hamstring strain injury. Frontiers in Physiology, 8. doi:10.3389/fphys.2017.00025

Romero-Rodriguez, D. (2010). Nuestra clave en la prevención de lesiones. In Prevención de lesiones en el deporte. Claves para un rendimiento deportivo óptimo (pp. 3-10). Madrid. Editorial Médica Panamericana.

Romero-Rodriguez, D., Gual, G., \& Tesch, P. A. (2011). Efficacy of an inertial resistance training paradigm in the treatment of patellar tendinopathy in athletes: A case-series study. Physical Therapy in Sport, 12(1), 43-48. doi:10.1016/j.ptsp.2010.10.003

Romero Rodrígez, D., \& Tous Fajardo, J. (2010). Prevención de lesiones en el deporte: claves para un rendimiento deportivo óptimo. Madrid. Editorial Médica Panamericana.

Rossi, A., Pappalardo, L., Cintia, P., Pedreschi, D., Iaia, M. F., \& Alberti, G. (2016).

The importance of GPS features to describe elite football training. Póster en VIII Congresso Nazionale aicerca e formazione applicate alle Scienze motorie e sportive. Roma. Italia.

Ruddy, J. D., Pollard, C. W., Timmins, R. G., Williams, M. D., Shield, A. J., \& Opar, 
D. A. (2016). Running exposure is associated with the risk of hamstring strain injury in elite Australian footballers. British Journal of Sports Medicine, 52(14) 1-11. doi:10.1136/bjsports-2016-096777

Rumpf, M. C., Lockie, R. G., Cronin, J. B., \& Jalilvand, F. (2016). Effect of Different Sprint Training Methods on Sprint Performance Over Various Distances: A Brief Review. Journal of Strength and Conditioning Research, 30(6), 1767-85. doi:10.1519/JSC.0000000000001245

Schache, A. G., Dorn, T. W., Williams, G. P., Brown, N. A. T., \& Pandy, M. G. (2014). Lower-limb muscular strategies for increasing running speed. The Journal of Orthopaedic and Sports Physical Therapy, 44(10), 813-824. doi:10.2519/jospt.2014.5433

Schache, A. G., Kim, H.-J., Morgan, D., \& Pandy, M. G. (2010). Hamstring muscle forces prior to and immediately following an acute sprinting-related muscle strain injury. Gait \& Posture, 32(1), 136-140. doi:10.1016/j.gaitpost.2010.03.006

Schache, A. G., Wrigley, T. V., Baker, R., \& Pandy, M. G. (2009). Biomechanical response to hamstring muscle strain injury. Gait \& Posture, 29(2), 332-338. doi:10.1016/j.gaitpost.2008.10.054

Schelling, X., \& Torres, L. (2016). Accelerometer Load Profiles for BasketballSpecific Drills in Elite Players. Journal of Sports Science and Medicine, 15 (4), 585-591.

Schmitt, B., Tim, T., \& McHugh, M. (2012). Hamstring injury rehabilitation and prevention of reinjury using lengthened state eccentric training: a new concept. International Journal of Sports Physical Therapy, 7(3), 333-341.

Schoenfeld, B. J., Contreras, B., Tiryaki-Sonmez, G., Wilson, J. M., Kolber, M. J., \& 
Peterson, M. D. (2015). Regional differences in muscle activation during hamstrings exercise. Journal of Strength and Conditioning Research, 29(1), 159-164. doi:10.1519/JSC.0000000000000598

Schuermans, J., Danneels, L., Van Tiggelen, D., Palmans, T., \& Witvrouw, E. (2017). Proximal Neuromuscular Control Protects Against Hamstring Injuries in Male Soccer Players: A Prospective Study With Electromyography Time-Series Analysis During Maximal Sprinting. The American Journal of Sports Medicine, 45(6), 1315-1325. doi:10.1177/0363546516687750

Schuermans, J., Van Tiggelen, D., \& Witvrouw, E. (2017). Prone Hip Extension Muscle Recruitment is Associated with Hamstring Injury Risk in Amateur Soccer. International Journal of Sports Medicine, 38(9), 696-706. doi:10.1055/s-0043-103016

Schutz, Y., \& Herren, R. (2000). Assessment of speed of human locomotion using a differential satellite global positioning system. Medicine and Science in Sports and Exercise, 32(3), 642-6.

Scott, B. R., Lockie, R. G., Knight, T. J., Clark, A. C., \& Janse de Jonge, X. A. K. (2013). A comparison of methods to quantify the in-season training load of professional soccer players. International Journal of Sports Physiology and Performance, 8(2), 195-202. doi:10.1123/ijspp.8.2.195.

Shankar, P. R., Fields, S. K., Collins, C. L., Dick, R. W., \& Comstock, R. D. (2007). Epidemiology of High School and Collegiate Football Injuries in the United States, 2005-2006. The American Journal of Sports Medicine, 35(8), 1295-1303. doi:10.1177/0363546507299745

Sherry, M. A., Johnston, T. S., \& Heiderscheit, B. C. (2015). Rehabilitation of Acute Hamstring Strain Injuries. Clinics in Sports Medicine, 34(2), 263-284. 
doi:10.1016/J.CSM.2014.12.009

Shield, A. J., \& Bourne, M. N. (2018). Hamstring Injury Prevention Practices in Elite Sport: Evidence for Eccentric Strength vs. Lumbo-Pelvic Training. Sports Medicine, 48(3), 513-524. doi:10.1007/s40279-017-0819-7

Sole, G., Milosavljevic, S., Nicholson, H., \& Sullivan, S. J. (2012). Altered muscle activation following hamstring injuries. British Journal of Sports Medicine, 46(2), 118-123. doi:10.1136/bjsm.2010.079343

Sonderegger, K., Tschopp, M., \& Taube, W. (2016). The challenge of evaluating the intensity of short actions in soccer: A new methodological approach using percentage acceleration. PLoS ONE, 11(11), 1-10. doi:10.1371/journal.pone.0166534

Spencer, M., Bishop, D., Dawson, B., \& Goodman, C. (2005). Physiological and metabolic responses of repeated-sprint activities:specific to field-based team sports. Sports Medicine. 35(12), 1025-1044.

Stagno, K. M., Thatcher, R., \& van Someren, K. A. (2007). A modified TRIMP to quantify the in-season training load of team sport players. Journal of Sports Sciences, 25(6), 629-634. doi:10.1080/02640410600811817

Stares, J., Dawson, B., Peeling, P., Drew, M., Heasman, J., Rogalski, B., \& Colby, M. (2018). How much is enough in rehabilitation? High running workloads following lower limb muscle injury delay return to play but protect against subsequent injury. Journal of Science and Medicine in Sport. 21(10) 1019-1024. doi:10.1016/j.jsams.2018.03.012

Stevens, T. G. A., De Ruiter, C. J., Twisk, J. W. R., Savelsbergh, G. J. P., \& Beek, P. J. (2017). Quantification of in-season training load relative to match load in professional Dutch Eredivisie football players. Science and Medicine in Football. 
1(2), 117-125. doi:10.1080/24733938.2017.1282163

Stewart, P. F., Turner, A. N., Miller, S. C., Stewart, P. F., Burroughs, T., \& Nw, L. (2014). Reliability, factorial validity , and interrelationships of five commonly used change of direction speed tests, 500-506. doi:10.1111/sms.12019

Stølen, T., Chamari, K., Castagna, C., \& Wisløff, U. (2005). Physiology of soccer: an update. Sports Medicine, 35(6), 501-536. doi:10.2165/00007256-20053506000004

Styles, W. J., Matthews, M. J., \& Comfort, P. (2016). Effects of Strength Training On Squat And Sprint Performance in Soccer Players. Journal of Strength \& Conditioning Research, 30(6), 1534-1539. doi:10.1519/JSC.0000000000001243

Suárez-Arrones, L., Nuñez, F. J., Portillo, J., \& Méndez-Villanueva, A. (2012). Running Demands and Heart Rate Responses in Men Rugby Sevens. Journal of Strength and Conditioning Research, 26(11), 3155-3159. doi:10.1519/JSC.0b013e318243fff7

Suárez-Arrones, L., Torreño, N., Requena, B., Sáez De Villarreal, E., Casamichana, D., Barbero-Alvarez, J. C., \& Munguía-Izquierdo, D. (2015a). Match-play activity profile in professional soccer players during official games and the relationship between external and internal load. The Journal of Sports Medicine and Physical Fitness, 55(12), 1417-22.

Suárez-Arrones, L., Torreño, N., Requena, B., Sáez De Villarreal, E., Casamichana, D., Barbero-Alvarez, J. C., \& Munguía-Izquierdo, D. (2015b). Match-play activity profile in professional soccer players during official games and the relationship between external and internal load. The Journal of Sports Medicine and Physical Fitness, 55(12), 1417-22. doi:R40Y9999N00A140099 [pii]

Sugiura, Y., Sakuma, K., Sakuraba, K., \& Sato, Y. (2017). Prevention of Hamstring 
Injuries in Collegiate Sprinters. Orthopaedic Journal of Sports Medicine, 5(1), 232596711668152. doi:10.1177/2325967116681524

Svensson, K., Alricsson, M., Eckerman, M., Magounakis, T., \& Werner, S. (2016). The correlation between the imaging characteristics of hamstring injury and time required before returning to sports: a literature review. Journal of Exercise Rehabilitation, 12(3), 134-42. doi:10.12965/jer.1632558.279

Swann, C., Moran, A., \& Piggott, D. (2015). Defining elite athletes: Issues in the study of expert performance in sport psychology. Psychology of Sport and Exercise, 16, 3-14. doi:10.1016/J.PSYCHSPORT.2014.07.004

Sweeting, A. J., Cormack, S. J., Morgan, S., \& Aughey, R. J. (2017). When Is a Sprint a Sprint? A Review of the Analysis of Team-Sport Athlete Activity Profile. Frontiers in Physiology, 8, 432. doi:10.3389/fphys.2017.00432

Taberner, M., Allen, T., \& Cohen, D. D. (2019). Progressing rehabilitation after injury: consider the 'control-chaos continuum.' British Journal of Sports Medicine, bjsports-2018-100157. doi:10.1136/bjsports-2018-100157

Taberner, M., \& Cohen, D. D. (2018). Physical preparation of the football player with an intramuscular hamstring tendon tear: clinical perspective with video demonstrations. British Journal of Sports Medicine, 52(19), 1275-1278. doi:10.1136/bjsports-2017-098817

Taylor, J., Macpherson, T., Spears, I., \& Weston, M. (2015). The Effects of RepeatedSprint Training on Field-Based Fitness Measures: A Meta-Analysis of Controlled and Non-Controlled Trials. Sports Medicine, 45(6), 881-891. doi:10.1007/s40279-015-0324-9

Taylor, Wright, A. A., Dischiavi, S. L., Townsend, M. A., \& Marmon, A. R. (2017). Activity Demands During Multi-Directional Team Sports: A Systematic Review. 
Sports Medicine, 47(12), 2533-2551. doi:10.1007/s40279-017-0772-5

Tee, J. C., Lambert, M. I., \& Coopoo, Y. (2016). GPS comparison of training activities and game demands of professional rugby union. International Journal of Sports Science \& Coaching, 11(2), 200-211. doi:10.1177/1747954116637153

Terrier, P., Turner, V., \& Schutz, Y. (2005). GPS analysis of human locomotion: Further evidence for long-range correlations in stride-to-stride fluctuations of gait parameters. Human Movement Science, 24(1), 97-115. doi:10.1016/j.humov.2005.03.002

Thelen, D. G., Chumanov, E. S., Best, T. M., Swanson, S. C., \& Heiderscheit, B. C. (2005). Simulation of biceps femoris musculotendon mechanics during the swing phase of sprinting. Medicine and Science in Sports and Exercise, 37(11), 19311938.

Thelen, D. G., Chumanov, E. S., Hoerth, D. M., Best, T. M., Swanson, S. C., Li, L., ... Heiderscheit, B. C. (2005). Hamstring muscle kinematics during treadmill sprinting. Medicine and Science in Sports and Exercise, 37(1), 108-14.

Thelen, D. G., Chumanov, E. S., Sherry, M. A., \& Heiderscheit, B. C. (2006). Neuromusculoskeletal models provide insights into the mechanisms and rehabilitation of hamstring strains. Exercise and Sport Sciences Reviews, 34(3), 135-41.

Tibana, R., de Sousa, N., Cunha, G., Prestes, J., Fett, C., Gabbett, T., \& Voltarelli, F. (2018). Validity of Session Rating Perceived Exertion Method for Quantifying Internal Training Load during High-Intensity Functional Training. Sports, 6(3), 68. doi:10.3390/sports6030068

Timmins, R. (2017). Biceps femoris architecture: the association with injury and response to training. British Journal of Sports Medicine, 51(6), 547-548. 
doi:10.1136/bjsports-2016-097044

Tol, J. L., Hamilton, B., Eirale, C., Muxart, P., Jacobsen, P., \& Whiteley, R. (2014). At return to play following hamstring injury the majority of professional football players have residual isokinetic deficits. British Journal of Sports Medicine, 48(18), 1364-1369. doi:10.1136/bjsports-2013-093016

Tominaga, R., Ishii, Y., Ueda, T., \& Kurokawa, T. (2016). The Effects of Running Speed on Ground Reaction Forces and Lower Limb Kinematics During SingleLeg Stop Movement. Journal of Strength and Conditioning Research, 30(5), 1224-1230. doi:10.1519/JSC.0000000000000286

Townshend, andrew d., Worringham, charles j., \& Stewart, ian b. (2008). Assessment of speed and position during human locomotion using nondifferential GPS. Medicine and Science in Sports and Exercise, 40(1), 124132. doi:10.1249/mss.0b013e3181590bc2

Tsaklis, P., Malliaropoulos, N., Mendiguchia, J., Korakakis, V., Tsapralis, K., Pyne, D., \& Malliaras, P. (2015). Muscle and intensity based hamstring exercise classification in elite female track and field athletes: implications for exercise selection during rehabilitation. Open Access Journal of Sports Medicine, 6, 20917. doi:10.2147/OAJSM.S79189

Tyler, T. F., Schmitt, B., Nicholas, S. J., \& McHugh, M. P. (2017). Rehabilitation After Hamstring-Strain Injury Emphasizing Eccentric Strengthening at Long Muscle Lengths: Results of Long-Term Follow-Up. Journal of Sport Rehabilitation, 26(2), 131-140. doi:10.1123/jsr.2015-0099

Urrutia Egaña, M., Barrios Araya, S., Marina Gutiérrez Núñez, L., \& Magdalena Mayorga Camus, L. (2014). Métodos óptimos para determinar validez de contenido. Educación Médica Superior, 28 (3), 547-558. 
Valera-Garrido, F., Minaya-Muñoz, F., \& Medina-Mirapeix, F. (2014). Ultrasoundguided percutaneous needle electrolysis in chronic lateral epicondylitis: shortterm and long-term results. Acupuncture in Medicine: Journal of the British Medical Acupuncture Society, 32(6), 446-54. doi:10.1136/acupmed-2014010619

Valle, X., Alentorn-Geli, E., Tol, J. L., Hamilton, B., Garrett, W. E., Pruna, R., ... Rodas, G. (2017). Muscle Injuries in Sports: A New Evidence-Informed and Expert Consensus-Based Classification with Clinical Application. Sports Medicine, 47(7), 1241-1253. doi:10.1007/s40279-016-0647-1

Valle, X., Mechó, S., Pruna, R., Pedret, C., Isern, J., Monllau, J. C., \& Rodas, G. (2018). The MLG-R muscle injury classification for hamstrings. Examples and guidelines for its use. Apunts. Medicina de l'Esport. In press. doi:10.1016/J.APUNTS.2018.11.002

Valle, X., Tol, J. L., Hamilton, B., Rodas, G., Malliaras, P., Malliaropoulos, N., ... Jardi, J. (2015). Hamstring muscle injuries, a rehabilitation protocol purpose. Asian Journal of Sports Medicine, 6(4), 1-11. doi:10.5812/asjsm.25411

Van Beijsterveldt, A. M. C., van de Port, I. G. L., Vereijken, A. J., \& Backx, F. J. (2013). Risk Factors for Hamstring Injuries in Male Soccer Players: A Systematic Review of Prospective Studies. Scandinavian Journal of Medicine \& Science in Sports, 23(3), 253-262. doi:10.1111/j.1600-0838.2012.01487.x

Van der Horst, N., Backx, F. J., Goedhart, E. A., \& Huisstede, B. M. A. (2017). Return to play after hamstring injuries in football (soccer): A worldwide Delphi procedure regarding definition, medical criteria and decision-making. British Journal of Sports Medicine 51(22), 1583-1591. doi:10.1136/bjsports-2016- 
097206

Van der Horst, N., van de Hoef, S., Reurink, G., Huisstede, B., \& Backx, F. J. (2016a). Return to Play After Hamstring Injuries: A Qualitative Systematic Review of Definitions and Criteria. Sports Medicine, 46(6), 899-912. doi:10.1007/s40279015-0468-7

Van der Horst, N., van de Hoef, S., Reurink, G., Huisstede, B., \& Backx, F. J. (2016b). Return to Play After Hamstring Injuries: A Qualitative Systematic Review of Definitions and Criteria. Sports Medicine, 46(6), 899-912. doi:10.1007/s40279015-0468-7

Van der Made, A. D., Almusa, E., Whiteley, R., Hamilton, B., Eirale, C., van Hellemondt, F., \& Tol, J. L. (2017). Intramuscular tendon involvement on MRI has limited value for predicting time to return to play following acute hamstring injury. British Journal of Sports Medicine, 52(2), 83-88. doi:10.1136/bjsports2017-097659

Van Hooren, B., \& Bosch, F. (2017). Is there really an eccentric action of the hamstrings during the swing phase of high-speed running? part I: A critical review of the literature. Journal of Sports Sciences, 35(23), 2313-2321. doi:10.1080/02640414.2016.1266018

Van Mechelen, W., Hlobil, H., \& Kemper, H. C. G. (1992). Incidence, Severity, Aetiology and Prevention of Sports Injuries: A Review of Concepts. Sports Medicine: An International Journal of Applied Medicine and Science in Sport and Exercise, 14(2), 82-99. doi:10.2165/00007256-199214020-00002

Varley, M. C., Fairweather, I. H., \& Aughey, R. J. (2012). Validity and reliability of GPS for measuring instantaneous velocity during acceleration, deceleration, and constant motion. Journal of Sports Sciences, 30(2), 121-127. 
doi:10.1080/02640414.2011.627941

Varley, M. C., Gabbett, T., \& Aughey, R. J. (2014). Activity profiles of professional soccer, rugby league and Australian football match play. Journal of Sports Sciences, 32(20), 1858-1866. doi:10.1080/02640414.2013.823227

Verrall, G., Slavotinek, J. P., Barnes, P., Fon, G. T., \& Spriggins, A. J. (2001). Clinical risk factors for hamstring muscle strain injury: a prospective study with correlation of injury by magnetic resonance imaging. British Journal of Sports Medicine, 35(6), 435-9

Verstegen, M., Falsone, S., Orr, R., \& Smith, S. (2012). Suggestions From the Field for Return to Sports Participation Following Anterior Cruciate Ligament Reconstruction: American Football. Journal of Orthopaedic \& Sports Physical Therapy, 42(4), 337-344. doi:10.2519/jospt.2012.4031

Wangensteen, A., Tol, J. L., Witvrouw, E., Van Linschoten, R., Almusa, E., Hamilton, B., \& Bahr, R. (2016). Hamstring Reinjuries Occur at the Same Location and Early After Return to Sport. The American Journal of Sports Medicine, 44(8), 2112-2121. doi:10.1177/0363546516646086

Wehbe, G. M., Hartwig, T. B., \& Duncan, C. S. (2014a). Movement analysis of Australian national league soccer players using global positioning system technology. Journal of Strength and Conditioning Research, 28(3), 834-842. doi:10.1519/JSC.0b013e3182a35dd1

Wehbe, G. M., Hartwig, T. B., \& Duncan, C. S. (2014b). Movement Analysis of Australian National League Soccer Players Using Global Positioning System Technology. Journal of Strength and Conditioning Research, 28(3), 834-842. doi:10.1519/JSC.0b013e3182a35dd1

White, A. D., \& MacFarlane, N. (2013). Time-on-pitch or full-game GPS analysis 
procedures for elite field hockey? International Journal of Sports Physiology and Performance, 8(5), 549-555.

Witvrouw, E., Danneels, L., Asselman, P., D’Have, T., \& Cambier, D. (2003). Muscle Flexibility as a Risk Factor for Developing Muscle Injuries in Male Professional Soccer Players. The American Journal of Sports Medicine, 31(1), 41-46. doi:10.1177/03635465030310011801

Woods, C., Hawkins, R. D., Maltby, S., Hulse, M., Thomas, A., \& Hodson, A. (2004). The Football Association Medical Research Programme: an audit of injuries in professional football--analysis of hamstring injuries. British Journal of Sports Medicine, 38(1), 36-41.

Zambaldi, M., Beasley, I., \& Rushton, A. (2017). Return to play criteria after hamstring muscle injury in professional football: a Delphi consensus study. British Journal of Sports Medicine, 51(16), 1221-1226. doi:10.1136/bjsports2016-097131

Zebis, M. K., Skotte, J., Andersen, C. H., Mortensen, P., Petersen, H. H., Viskær, T. C., ... Andersen, L. L. (2013). Kettlebell swing targets semitendinosus and supine leg curl targets biceps femoris: an EMG study with rehabilitation implications. British Journal of Sports Medicine, 47(18), 1192-1198. doi:10.1136/bjsports-2011-090281 

VIII. Anexos 



\subsection{Permisos para la toma de datos}

\section{Getafe CF SAD}

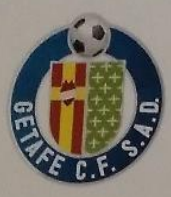

To whomsoever it may concern:

The study titled "Validity and reliability of a training program for the readaptation and retraining phase following an injury to the intramuscular myotendinous junction proximal of biceps femoris in professional soccer players" by Jiménez Rubio, Navandar, Rivilla-García and Paredes was carried with players of Getafe Club de Fútbol Sociedad Anónima Deportiva. All players who participated in the study provided written informed consent in accordance with the departmental ethical procedures and following the principles outlined in the Declaration of Helsinki.

In Madrid, on the $1^{\text {st }}$ of November, 2017.

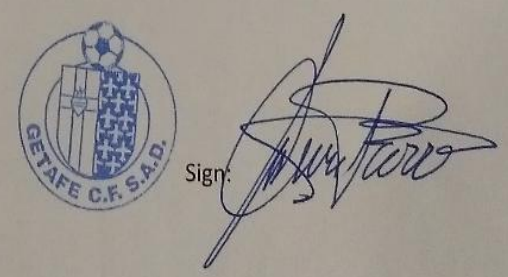

Figura 45. Justificación toma de Datos con Getafe CF, validación intervención 


\section{Rayo Vallecano de Madrid SAD}

\section{$\underline{\text { To whomsoever it may concern: }}$}

The study titled "Validity and reliability of a training program for the readaptation and retraining phase following an injury to the intramuscular myotendinous junction proximal of biceps femoris in professional soccer players" by Jiménez Rubio, Navandar, Rivilla-García and ParedesHernández was carried with players of Rayo Vallecano de Madrid Sociedad Anónima Deportiva. All players who participated in the study provided written informed consent in accordance with the departmental ethical procedures and following the principles outlined in the Declaration of Helsinki.

In Madrid, on the $1^{\text {st }}$ of November, 2017.

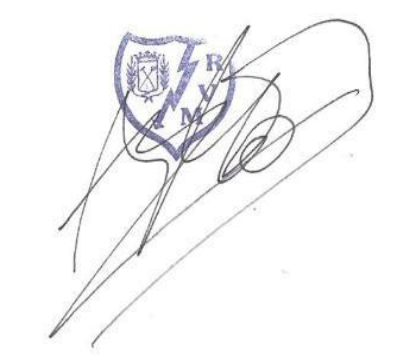

C/ PAYASO FOFÓ, s/n. - 28018 MADRID - Teléfonos: 9147822 53/91478 56 72/91478 23.63 - Fax: 914771754 www.rayovallecano.es

Figura 46. Justificación toma de datos con Rayo Vallecano de Madrid, validación intervención 


\subsection{Comité de Ética}

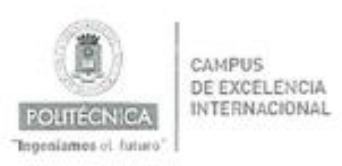

\section{INFORME QUE EMITE EL COMITÉ DE ÉTICA} DE LA UNIVERSIDAD POLITÉCNICA DE MADRID

A petición de la Vicerrectora de Investigación, Innovación y Doctorado de la Universidad Politécnica de Madrid,

Da Asunción Gómez Pérez, de un dictamen sobre los aspectos éticos del

Proyecto "Análisis de los efectos de un programa de readaptación y reentrenamiento en futbolistas profesionales que sufrieron lesión en la unión miotendinosa proximal del biceps femoral" cuyo investigador principal es D. JESUS RIVILLA GARCIA, del Departamento de deportes / Grupo de Investigación: Deporte y Rendimiento, en la Facultad de Ciencias de la Actividad Fisica y del Deporte (INEF/UPM).

El solicitante declara conocer los principios éticos y las normas legales que rigen las actividades de investigación y se compromete a respetar estos principios y normas en el desarrollo del estudio propuesto. Igualmente, se compromete a no modificar los protocolos de investigación y a solicitar una nueva autorización en caso de modificación.

El solicitante declara conocer la legislación relativa a la protección de datos y se compromete a respetar la confidencialidad de los datos personales de este estudio, así como a hacer explicito este compromiso ante las personas que participan en el proyecto.

El solicitante declara conocer la legislación descrita en la Ley Orgánica 15/1999, de 13 de diciembre, sobre la Protección de Datos de Carácter Personal.

Real Decreto 1720/2007 de 21 de diciembre, por el que se aprueba el Reglamento de desarrollo de la Ley orgánica 15/1999 (BOE n 17 de 19 de enero de 2008, pág. 4103-4136). Ley $41 / 2002$, de 14 de noviembre, ley básica reguladora de la autonomía del paciente y de derechos $y$ obligaciones en materia de informaciön $y$ documentación clínica, y se compromete a cumplirlas.

Por lo tanto, no hay inconveniente en informar favorablemente la propuesta, recomendando el efectivo cumplimiento de los compromisos declarados.

Y para que así conste, firmo el presente informe en Madrid a 27 de abril de dos mil dieciocho.

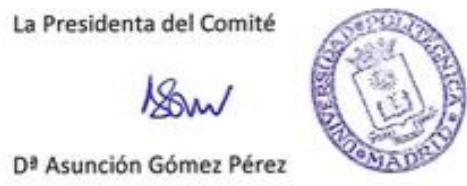

Dà Asunción Gómez Pérez

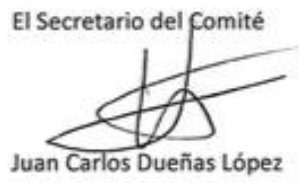

Figura 47. Documento favorable del comité de ética (Datos) 


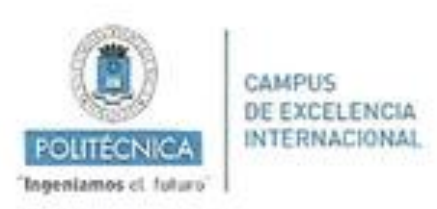

* Así mismo, el solicitante declara conocer la legislación relativa a la protección de datos, en particular la Ley Orgánica 15/1999, sobre la Protección de Datos de Carácter Personal y el R. D. $1720 / 2007$ de desarrollo, y se compromete a respetar la confidencialidad de los datos personales de este estudio, asi como a hacer explicito este compromiso ante las personas que participan en el proyecto.

* Igualmente, se compromete a no modificar los protocolos de investigación y a solicitar una nueva autorización en caso de modificación.

Por lo tanto, no hay inconveniente en informar favorablemente la propuesta, recomendando el efectivo cumplimiento de los compromisos declarados.

Y para que asi conste, firmo el presente informe en Madrid a 2 de julio de dos mil dieciocho.

La Presidenta del Comité

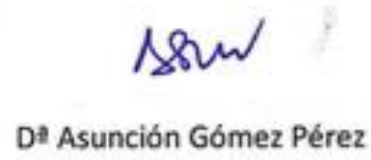

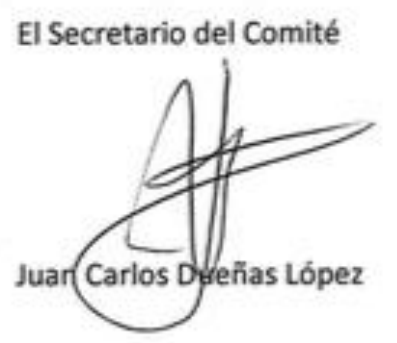

Figura 48. Documento favorable del comité de ética (Humanos) 


\subsection{Consentimiento informado para los participantes de la investigación}

El propósito de esta ficha de consentimiento es proveer a los participantes en esta investigación con una clara explicación de la naturaleza de la misma, así como de su rol en ella como participantes.

La presente investigación es conducida por_Sergio Jiménez Rubio, con DNI 70986134-S, doctorando de la Universidad Politécnica de Madrid. La meta de este estudio es desarrollar una Tesis Doctoral perteneciente a la Facultad de Ciencias de la Actividad Física y deporte (UPM -Madrid)

Si usted accede a participar en este estudio, se le pedirá entrenar y competir con un dispositivo GPS colocado en su espalda, a la altura de la charnela cervicodorsal, a través de un chaleco específico y adaptado para la colocación del dispositivo. Tras los entrenamientos y/o competiciones donde se registren datos y variables para el estudio en cuestión, se volcarán a un software y dichos datos serán confidenciales, con un único uso, la investigación, y el análisis de los mismos por los investigadores y preparadores físicos del club en cuestión.

La participación es este estudio es estrictamente voluntaria. La información que se recoja será confidencial y no se usará para ningún otro propósito fuera de los de esta investigación, y el análisis para mejorar el rendimiento físico-deportivo de los deportistas evaluados, única y exclusivamente por los técnicos del club en cuestión.

Si tiene alguna duda sobre este proyecto, puede hacer preguntas en cualquier momento durante su participación en él. Igualmente, puede retirarse del proyecto en cualquier momento sin que eso lo perjudique en ninguna forma. Si en alguna de las tareas de entrenamiento, o situaciones competitivas le parecen incómodas llevando el dispositivo, tiene usted el derecho de hacérselo saber al investigador y de no llevar el 
GPS en su espalda.

Le agradecemos su participación.

Acepto participar voluntariamente en esta investigación, conducida por Sergio Jiménez Rubio. He sido informado de que la meta de este estudio es: Obtener parámetros de carga externa a través del GPS para evaluar parámetros de rendimiento en diferentes momentos, y con ello para investigar acerca de la situación en cada uno de los momentos del presente estudio.

Reconozco que la información que yo provea en el curso de esta investigación es estrictamente confidencial y no será usada para ningún otro propósito fuera de los de este estudio sin mi consentimiento.

He sido informado de que puedo hacer preguntas sobre el proyecto en cualquier momento y que puedo retirarme del mismo cuándo así lo decida, sin que esto acarree perjuicio alguno para mi persona. De tener preguntas sobre mi participación en este estudio, puedo contactar a Sergio Jiménez Rubio al teléfono

Entiendo que una copia de esta ficha de consentimiento me será entregada, y que puedo pedir información sobre los resultados de este estudio cuándo éste haya concluido. Para esto, puedo contactar a SERGIO JIMÉNEZ RUBIO al teléfono anteriormente mencionado.

Nombre del Participante

Firma del Participante

Fecha

(en letras de imprenta) 


\subsection{Validación del programa de readaptación y reentrenamiento}

"Validity of an on-Field Readaptation Program Following a Hamstring Injury in Professional Soccer" by Jiménez-Rubio S, Navandar A, Rivilla-García J, Paredes-Hernández V

Journal of Sport Rehabilitation

(e) 2018 Human Kinetics, Inc.

Note: This article will be published in a forthcoming issue of the Journal of Sport Rehabilitation. The article appears here in its accepted, peer-reviewed form, as it was provided by the submitting author. It has not been copyedited, proofed, or formatted by the publisher.

Section: Technical Report

Article Title: Validity of an on-Field Readaptation Program Following a Hamstring Injury in Professional Soccer

Authors: Sergio Jiménez-Rubio ${ }^{\mathrm{a}}$; Archit Navandar ${ }^{\mathrm{a}}$, Jesús Rivilla-García ${ }^{\mathrm{a}}$; and Victor Paredes-Hernández ${ }^{\mathrm{b}}$

Affiliations: a Departamento de Deportes - Sports Department, Universidad Politécnica de Madrid, Madrid, Spain. ${ }^{b}$ Camilo José Cela University. Faculty of Physical Activity and Sports Sciences Calle Castillo de Alarcón, Madrid, Spain.

Running Head: On-field HIS readaptation program for soccer

Journal: Journal of Sport Rehabilitation

Acceptance Date: October 14, 2018

(92018 Human Kinetics, Inc.

DOI: https://doi.org/10.1123/jsr.2018-0203 


\subsection{Programa de entrenamiento (Jiménez-Rubio et al., 2018).}

\begin{tabular}{|c|c|c|}
\hline No. & Description & $\begin{array}{l}\text { Total } \\
\text { time }\end{array}$ \\
\hline 1 & $\begin{array}{l}\text { Lateral displacements; and alternative single-legged dead-lifts and } \\
\text { controlled landing drills with lowering of the centre of gravity at } \\
\text { controlled velocities and leverages of the stretch-shortening cycle of the } \\
\text { hamstrings (Mendiguchia et al., 2017; Schuermans, Danneels, Van } \\
\text { Tiggelen, Palmans, \& Witvrouw, 2017). }\end{array}$ & $3 \min$ \\
\hline 2 & $\begin{array}{l}\text { Coordinated soccer-specific controlled drills (Anemaet \& } \\
\text { Hammerich, 2014) at the static and dynamic level: ball control, passing, } \\
\text { dribbling and kicking. }\end{array}$ & $6 \min$ \\
\hline 3 & $\begin{array}{l}6 \text { discontinuous aerobic running drills (under } 14 \mathrm{~km} / \mathrm{h}) \text { and re- } \\
\text { education of sagital acceleration patterns }(7 \mathrm{~s} \text { of acceleration and } 3 \mathrm{~s} \text { of } \\
\text { deceleration). }\end{array}$ & $1 \mathrm{~min}$ \\
\hline 4 & $\begin{array}{l}3 \text { sets of soccer-specific agility and coordination drills (with and } \\
\text { without a ball in the same action) followed by a return to the initial } \\
\text { position walking }\end{array}$ & $1 \mathrm{~min} 10 \mathrm{~s}$ \\
\hline
\end{tabular}




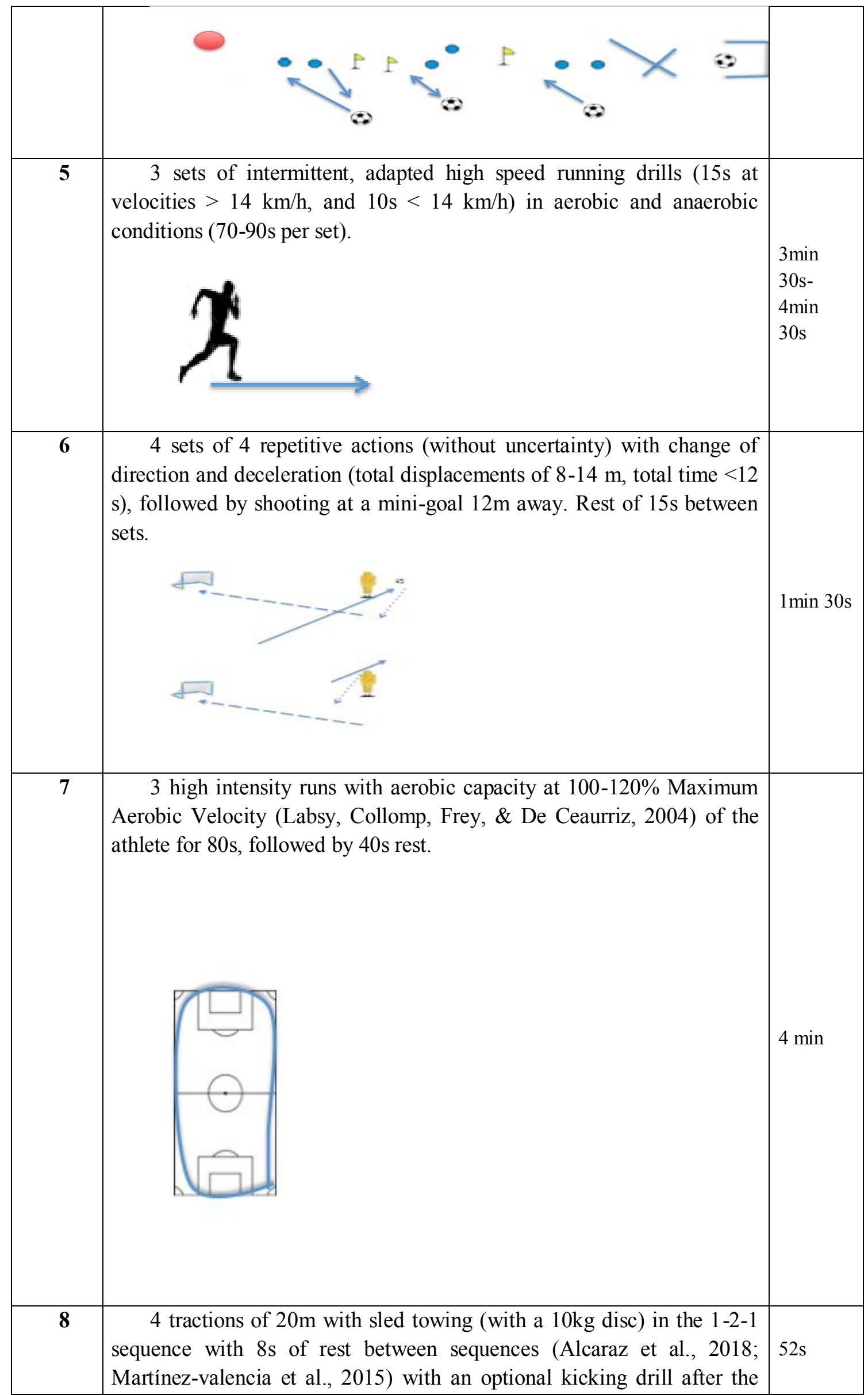




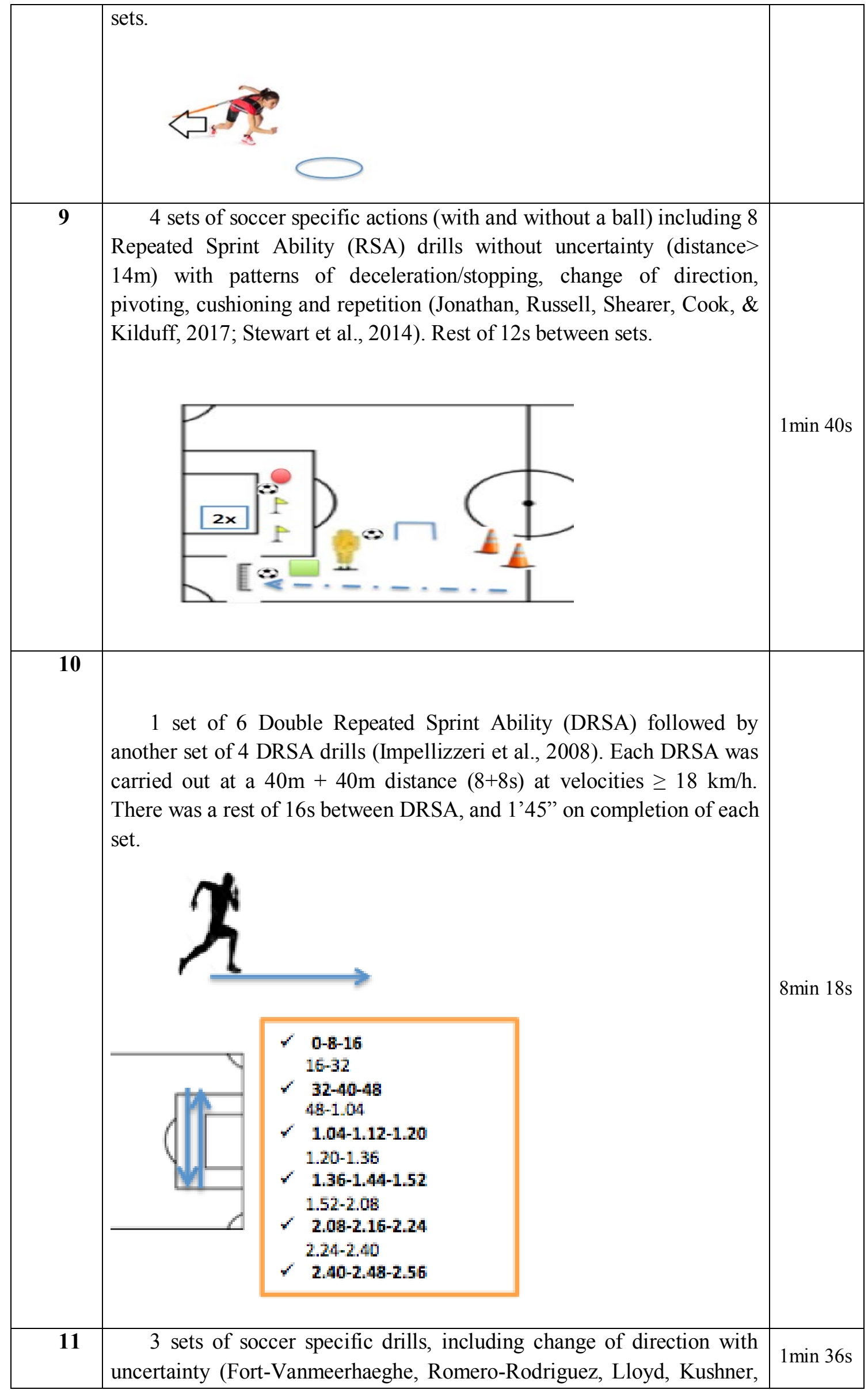




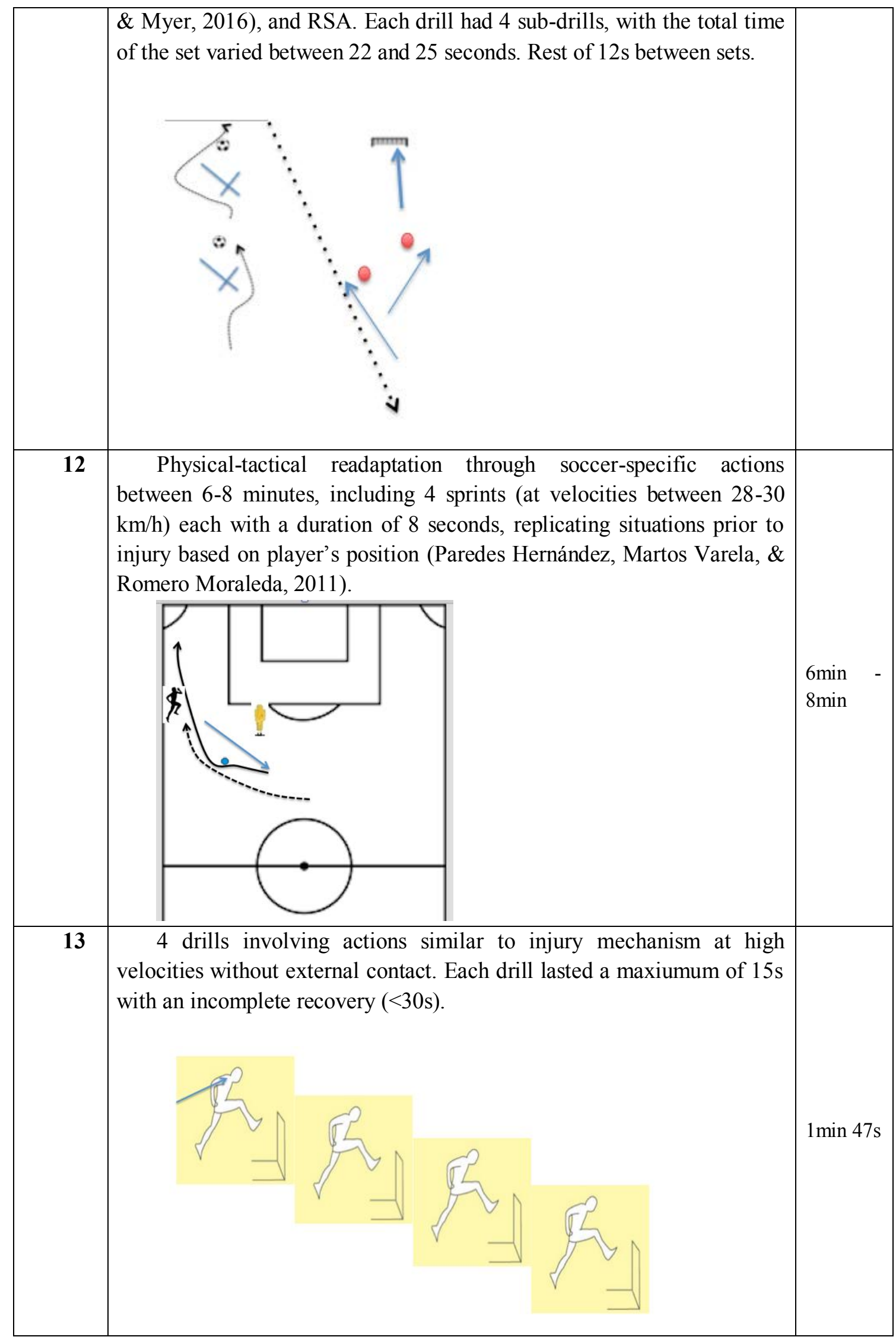




\subsection{Perfil de expertos (Jiménez-Rubio et al., 2018)}

\begin{tabular}{|c|c|c|}
\hline \multicolumn{3}{|l|}{ Highest Academic Qualifications } \\
\hline Degree & Number & $\%$ \\
\hline $\mathrm{PhD}$ & 8 & 53,33 \\
\hline Master's & 4 & 26,66 \\
\hline Graduate (Sports Science) & 3 & 20 \\
\hline \multicolumn{3}{|l|}{ Other Complementary Academic Qualifications } \\
\hline Degree & Number & $\%$ \\
\hline Physiotherapist (Graduate) & 4 & 26,6 \\
\hline Master's in Readaptation + Physiotherapy & 3 & 20 \\
\hline Master's in Personal Training + Master's in Readaptation & 6 & 40 \\
\hline No complementary qualifications (only Sports Science Degree) & 2 & 13,33 \\
\hline \multicolumn{3}{|l|}{ Soccer Specific Professional Qualifications } \\
\hline Level of License & Number & $\%$ \\
\hline UEFA Pro & 3 & 20 \\
\hline UEFA A & 4 & 26,66 \\
\hline UEFA B & 3 & 20 \\
\hline Without license & 5 & 33,33 \\
\hline \multicolumn{3}{|l|}{ Experience with injured players } \\
\hline Profile & Number & $\%$ \\
\hline As S \& C Coach $>10$ seasons & 2 & 13,33 \\
\hline As Rehab S \& C Coach 10-15 seasons with elite athletes & 3 & 20 \\
\hline As Rehab S \& C Coach $>15$ seasons with elite athletes & 10 & 66,66 \\
\hline \multicolumn{3}{|l|}{ Cumulative Experience in career (Titles) } \\
\hline Championship & Seasons & Titles \\
\hline UEFA Champions League & 3 & 2 \\
\hline UEFA Europa League & 14 & 1 \\
\hline 1st Division & 211 & 5 Leagues +3 Cups \\
\hline 2nd Division & 5 & \\
\hline
\end{tabular}



